\title{
Galactal Glycosylation and 2-Nitrogalactal Concatenation, a Powerful Combination for Mucin Core Structure Synthesis
}

\section{Supporting Information}

Jürgen Geiger, B. Gopal Reddy, Gottfried A. Winterfeld, R. R. Schmidt*

Fachbereich Chemie, Universität Konstanz, Fach M 725, D - 78457 Konstanz, Germany

Richard.Schmidt@uni-konstanz.de

Table of content

${ }^{1} \mathrm{H}$ NMR of compound $3 \quad$ S4

${ }^{13} \mathrm{C}$ NMR of compound $3 \quad$ S4

${ }^{1} \mathrm{H}$ NMR of compound $4\left(\mathrm{CDCl}_{3}, 250 \mathrm{MHz}\right) \quad$ S5

${ }^{1} \mathrm{H}$ NMR of compound $5\left(\mathrm{CDCl}_{3}, 250 \mathrm{MHz}\right) \quad$ S5

${ }^{1} \mathrm{H}$ NMR of compound $6\left(\mathrm{CDCl}_{3}, 250 \mathrm{MHz}\right) \quad$ S6

${ }^{1} \mathrm{H} \mathrm{NMR}$ of compound $\mathbf{8 a}\left(\mathrm{CDCl}_{3}, 250 \mathrm{MHz}\right) \quad$ S6

${ }^{13} \mathrm{C}$ NMR of compound $8 \mathbf{a}\left(\mathrm{CDCl}_{3}, 151 \mathrm{MHz}\right) \quad \mathrm{S} 7$

${ }^{1} \mathrm{H}$ NMR of compound 9a $\left(\mathrm{CDCl}_{3}, 600 \mathrm{MHz}\right) \quad$ S7

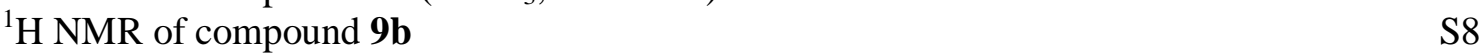

${ }^{13} \mathrm{C}$ NMR of compound $\mathbf{9 b}\left(\mathrm{CDCl}_{3}, 151 \mathrm{MHz}\right) \quad$ S8

${ }^{1} \mathrm{H}$ NMR of compound $\mathbf{1 1}\left(\mathrm{CDCl}_{3}, 250 \mathrm{MHz}\right) \quad$ S9

${ }^{13} \mathrm{C}$ NMR of compound $\mathbf{1 1}\left(\mathrm{CDCl}_{3}, 151 \mathrm{MHz}\right) \quad \mathrm{S} 9$

${ }^{1} \mathrm{H}$ NMR of compound $\mathbf{1 2}\left(\mathrm{CDCl}_{3}, 600 \mathrm{MHz}\right) \quad \mathrm{S} 10$

${ }^{13} \mathrm{C}$ NMR of compound $\mathbf{1 2}\left(\mathrm{CDCl}_{3}, 151 \mathrm{MHz}\right) \quad \mathrm{S} 10$

${ }^{1} \mathrm{H}$ NMR of compound $13\left(\mathrm{CDCl}_{3}, 600 \mathrm{MHz}\right) \quad \mathrm{S} 11$

${ }^{13} \mathrm{C}$ NMR of compound $\mathbf{1 3}\left(\mathrm{CDCl}_{3}, 151 \mathrm{MHz}\right) \quad \mathrm{S} 11$

${ }^{1} \mathrm{H}$ NMR of compound $14\left(\mathrm{CDCl}_{3}, 600 \mathrm{MHz}\right) \quad \mathrm{S} 12$

${ }^{1} \mathrm{H}$ NMR of compound $\mathbf{1 6} \alpha\left(\mathrm{CDCl}_{3}, 250 \mathrm{MHz}\right) \quad \mathrm{S} 12$

${ }^{13} \mathrm{C}$ NMR of compound $\mathbf{1 6} \alpha\left(\mathrm{CDCl}_{3}, 151 \mathrm{MHz}\right) \quad \mathrm{S} 13$

${ }^{1} \mathrm{H}$ NMR of compound $17\left(\mathrm{CDCl}_{3}, 600 \mathrm{MHz}\right) \quad \mathrm{S} 13$

${ }^{13} \mathrm{C}$ NMR of compound $17\left(\mathrm{CDCl}_{3}, 151 \mathrm{MHz}\right) \quad \mathrm{S} 14$

${ }^{1} \mathrm{H}$ NMR of compound $\mathbf{1 8}\left(\mathrm{CDCl}_{3}, 250 \mathrm{MHz}\right) \quad \mathrm{S} 14$

${ }^{13} \mathrm{C}$ NMR of compound $18\left(\mathrm{CDCl}_{3}, 63 \mathrm{MHz}\right) \quad \mathrm{S} 15$

${ }^{1} \mathrm{H}$ NMR of compound $19\left(\mathrm{CDCl}_{3}, 250 \mathrm{MHz}\right) \quad \mathrm{S} 15$

${ }^{13} \mathrm{C}$ NMR of compound $19\left(\mathrm{CDCl}_{3}, 63 \mathrm{MHz}\right) \quad \mathrm{S} 16$

${ }^{1} \mathrm{H}$ NMR of compound $20\left(\mathrm{CDCl}_{3}, 250 \mathrm{MHz}\right) \quad \mathrm{S} 16$

${ }^{1} \mathrm{H}$ NMR of compound 21a $\left(\mathrm{CDCl}_{3}, 600 \mathrm{MHz}\right) \quad \mathrm{S} 17$

${ }^{13} \mathrm{C}$ NMR of compound 21a $\left(\mathrm{CDCl}_{3}, 151 \mathrm{MHz}\right) \quad \mathrm{S} 17$

${ }^{1} \mathrm{H}$ NMR of compound 22a $\left(\mathrm{CDCl}_{3}, 600 \mathrm{MHz}\right) \quad \mathrm{S} 18$

${ }^{13} \mathrm{C}$ NMR of compound 22a $\left(\mathrm{CDCl}_{3}, 151 \mathrm{MHz}\right) \quad \mathrm{S} 18$

${ }^{1} \mathrm{H}$ NMR of compound 23a $\left(\mathrm{CDCl}_{3}, 250 \mathrm{MHz}\right) \quad \mathrm{S} 19$

${ }^{13} \mathrm{C}$ NMR of compound 23a $\left(\mathrm{CDCl}_{3}, 63 \mathrm{MHz}\right) \quad \mathrm{S} 19$

${ }^{1} \mathrm{H}$ NMR of compound 23b $\left(\mathrm{CDCl}_{3}, 600 \mathrm{MHz}\right) \quad$ S20

${ }^{13} \mathrm{C}$ NMR of compound 23b $\left(\mathrm{CDCl}_{3}, 151 \mathrm{MHz}\right) \quad$ S21

${ }^{1} \mathrm{H}$ NMR of compound 24a $\left(\mathrm{CDCl}_{3}, 250 \mathrm{MHz}\right) \quad$ S21

${ }^{13} \mathrm{C}$ NMR of compound 24a $\left(\mathrm{CDCl}_{3}, 63 \mathrm{MHz}\right) \quad$ S22

${ }^{1} \mathrm{H}$ NMR of compound 24b $\left(\mathrm{CDCl}_{3}, 250 \mathrm{MHz}\right) \quad$ S22

${ }^{1} \mathrm{H}$ NMR of compound $25 \mathrm{~A}\left(\mathrm{CDCl}_{3}, 600 \mathrm{MHz}\right) \quad$ S23

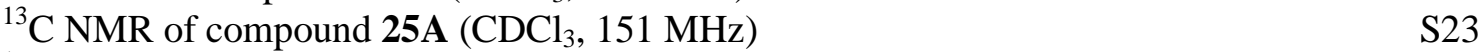

${ }^{1} \mathrm{H}$ NMR of compound 25B $\left(\mathrm{CDCl}_{3}, 250 \mathrm{MHz}\right) \quad$ S24 
${ }^{1} \mathrm{H}$ NMR of compound 25C $\left(\mathrm{CDCl}_{3}, 250 \mathrm{MHz}\right) \quad$ S25

${ }^{1} \mathrm{H} \mathrm{NMR}$ of compound $26\left(\mathrm{CDCl}_{3}, 600 \mathrm{MHz}\right) \quad$ S25

${ }^{13} \mathrm{C}$ NMR of compound $26\left(\mathrm{CDCl}_{3}, 151 \mathrm{MHz}\right) \quad$ S26

${ }^{1} \mathrm{H}$ NMR of compound $27\left(\mathrm{CDCl}_{3}, 250 \mathrm{MHz}\right) \quad$ S26

${ }^{13} \mathrm{C}$ NMR of compound $27\left(\mathrm{CDCl}_{3}, 63 \mathrm{MHz}\right) \quad$ S27

${ }^{1} \mathrm{H}$ NMR of compound 28a $\left(\mathrm{CDCl}_{3}, 600 \mathrm{MHz}\right) \quad$ S27

${ }^{13} \mathrm{C}$ NMR of compound 28a $\left(\mathrm{CDCl}_{3}, 151 \mathrm{MHz}\right) \quad$ S28

${ }^{1} \mathrm{H}$ NMR of compound $\mathbf{3 0}\left(\mathrm{CDCl}_{3}, 250 \mathrm{MHz}\right) \quad$ S28

${ }^{1} \mathrm{H} \mathrm{NMR}$ of compound $\mathbf{3 1}\left(\mathrm{CDCl}_{3}, 250 \mathrm{MHz}\right) \quad$ S29

${ }^{1} \mathrm{H}$ NMR of compound 33a $\left(\mathrm{CDCl}_{3}, 600 \mathrm{MHz}\right) \quad$ S29

${ }^{13} \mathrm{C}$ NMR of compound 33a $\left(\mathrm{CDCl}_{3}, 151 \mathrm{MHz}\right) \quad \mathrm{S} 30$

${ }^{1} \mathrm{H}$ NMR of compound 33b $\left(\mathrm{CDCl}_{3}, 600 \mathrm{MHz}\right) \quad \mathrm{S} 30$

${ }^{13} \mathrm{C}$ NMR of compound 33b $\left(\mathrm{CDCl}_{3}, 151 \mathrm{MHz}\right) \quad \mathrm{S} 31$

${ }^{1} \mathrm{H}$ NMR of compound 34a $\left(\mathrm{CDCl}_{3}, 600 \mathrm{MHz}\right) \quad \mathrm{S} 31$

${ }^{13} \mathrm{C}$ NMR of compound 34a $\left(\mathrm{CDCl}_{3}, 151 \mathrm{MHz}\right) \quad \mathrm{S} 32$

${ }^{1} \mathrm{H}$ NMR of compound 34b $\left(\mathrm{CDCl}_{3}, 600 \mathrm{MHz}\right) \quad \mathrm{S} 32$

${ }^{13} \mathrm{C}$ NMR of compound $\mathbf{3 4 b}\left(\mathrm{CDCl}_{3}, 151 \mathrm{MHz}\right) \quad$ S33

${ }^{1} \mathrm{H}$ NMR of compound 35a $\left(\mathrm{CDCl}_{3}, 600 \mathrm{MHz}\right) \quad \mathrm{S} 33$

${ }^{13} \mathrm{C}$ NMR of compound 35a $\left(\mathrm{CDCl}_{3}, 151 \mathrm{MHz}\right) \quad \mathrm{S} 34$

${ }^{1} \mathrm{H} \mathrm{NMR}$ of compound 35b $\left(\mathrm{CDCl}_{3}, 600 \mathrm{MHz}\right) \quad \mathrm{S} 34$

${ }^{13} \mathrm{C}$ NMR of compound 35b $\left(\mathrm{CDCl}_{3}, 151 \mathrm{MHz}\right) \quad \mathrm{S} 35$

${ }^{1} \mathrm{H} \mathrm{NMR}$ of compound $37\left(\mathrm{CDCl}_{3}, 600 \mathrm{MHz}\right) \quad \mathrm{S} 35$

${ }^{13} \mathrm{C}$ NMR of compound $37\left(\mathrm{CDCl}_{3}, 151 \mathrm{MHz}\right) \quad$ S36

${ }^{1} \mathrm{H}$ NMR of compound 38a $\left(\mathrm{CDCl}_{3}, 600 \mathrm{MHz}\right) \quad \mathrm{S} 37$

${ }^{13} \mathrm{C}$ NMR of compound 38a $\left(\mathrm{CDCl}_{3}, 151 \mathrm{MHz}\right) \quad \mathrm{S} 37$

${ }^{1} \mathrm{H}$ NMR of compound $\mathbf{3 8 b}\left(\mathrm{CDCl}_{3}, 600 \mathrm{MHz}\right) \quad \mathrm{S} 38$

${ }^{13} \mathrm{C}$ NMR of compound $\mathbf{3 8 b}\left(\mathrm{CDCl}_{3}, 151 \mathrm{MHz}\right) \quad \mathrm{S} 38$

${ }^{11} \mathrm{H}$ NMR of compound 39a $\left(\mathrm{CDCl}_{3}, 600 \mathrm{MHz}\right) \quad$ S39

${ }^{13} \mathrm{C}$ NMR of compound 39a $\left(\mathrm{CDCl}_{3}, 151 \mathrm{MHz}\right) \quad$ S39

${ }^{1} \mathrm{H}$ NMR of compound $39 \mathrm{~b}\left(\mathrm{CDCl}_{3}, 600 \mathrm{MHz}\right) \quad \mathrm{S} 40$

${ }^{13} \mathrm{C}$ NMR of compound 39b $\left(\mathrm{CDCl}_{3}, 151 \mathrm{MHz}\right)$

${ }^{1} \mathrm{H}$ NMR of compound 40a $\left(\mathrm{CDCl}_{3}, 600 \mathrm{MHz}\right) \quad \mathrm{S} 41$

${ }^{13} \mathrm{C}$ NMR of compound 40a $\left(\mathrm{CDCl}_{3}, 151 \mathrm{MHz}\right) \quad \mathrm{S} 41$

${ }^{1} \mathrm{H}$ NMR of compound 41a $\left(\mathrm{CDCl}_{3}, 600 \mathrm{MHz}\right) \quad \mathrm{S} 42$

${ }^{1} \mathrm{H}$ NMR of Boc-protected intermediate to $42 \mathrm{a}\left(\mathrm{CDCl}_{3}, 600 \mathrm{MHz}\right) \quad \mathrm{S} 43$

${ }^{1} \mathrm{H}$ NMR of compound $\mathbf{4 2 a}\left(\mathrm{CDCl}_{3}, 600 \mathrm{MHz}\right) \quad \mathrm{S} 43$

${ }^{13} \mathrm{C}$ NMR of compound 42a $\left(\mathrm{CDCl}_{3}, 151 \mathrm{MHz}\right) \quad \mathrm{S} 44$

${ }^{13} \mathrm{C}$ NMR of compound $43\left(\mathrm{CDCl}_{3}, 151 \mathrm{MHz}\right) \quad \mathrm{S} 45$

${ }^{1} \mathrm{H}$ NMR of compound $\mathbf{4 4}\left(\mathrm{CDCl}_{3}, 250 \mathrm{MHz}\right) \quad \mathrm{S} 45$

${ }^{13} \mathrm{C}$ NMR of compound $44\left(\mathrm{CDCl}_{3}, 151 \mathrm{MHz}\right) \quad \mathrm{S} 46$

${ }^{1} \mathrm{H}$ NMR of compound $\mathbf{4 5}\left(\mathrm{CDCl}_{3}, 600 \mathrm{MHz}\right) \quad \mathrm{S} 46$

${ }^{13} \mathrm{C}$ NMR of compound $45\left(\mathrm{CDCl}_{3}, 151 \mathrm{MHz}\right) \quad \mathrm{S} 47$

${ }^{1} \mathrm{H}$ NMR of compound $\mathbf{4 6 a}\left(\mathrm{CDCl}_{3}, 600 \mathrm{MHz}\right) \quad$ S47

${ }^{13} \mathrm{C}$ NMR of compound 46a $\left(\mathrm{CDCl}_{3}, 151 \mathrm{MHz}\right) \quad \mathrm{S} 48$

${ }^{1} \mathrm{H}$ NMR of compound 47a $\left(\mathrm{CDCl}_{3}, 600 \mathrm{MHz}\right) \quad \mathrm{S} 48$

${ }^{13} \mathrm{C}$ NMR of compound $47 \mathbf{a}\left(\mathrm{CDCl}_{3}, 151 \mathrm{MHz}\right) \quad$ S49

${ }^{1} \mathrm{H}$ NMR of compound $\mathbf{5 0}\left(\mathrm{CDCl}_{3}, 600 \mathrm{MHz}\right) \quad$ S50

${ }^{13} \mathrm{C}$ NMR of compound $\mathbf{5 0}\left(\mathrm{CDCl}_{3}, 151 \mathrm{MHz}\right) \quad$ S50

${ }^{1} \mathrm{H}$ NMR of compound $\mathbf{5 1}\left(\mathrm{CDCl}_{3}, 600 \mathrm{MHz}\right) \quad$ S51

${ }^{13} \mathrm{C}$ NMR of compound $\mathbf{5 1}\left(\mathrm{CDCl}_{3}, 151 \mathrm{MHz}\right) \quad \mathrm{S} 51$

${ }^{1} \mathrm{H}$ NMR of compound $\mathbf{5 2}\left(\mathrm{CDCl}_{3}, 600 \mathrm{MHz}\right) \quad$ S52 
${ }^{13} \mathrm{C}$ NMR of compound $\mathbf{5 2}\left(\mathrm{CDCl}_{3}, 151 \mathrm{MHz}\right) \quad$ S52

${ }^{1} \mathrm{H}$ NMR of compound $\mathbf{5 3}\left(\mathrm{CDCl}_{3}, 600 \mathrm{MHz}\right) \quad$ S53

${ }^{13} \mathrm{C}$ NMR of compound $\mathbf{5 3}\left(\mathrm{CDCl}_{3}, 151 \mathrm{MHz}\right) \quad$ S53

${ }^{1} \mathrm{H}$ NMR of compound $54\left(\mathrm{CDCl}_{3}, 600 \mathrm{MHz}\right) \quad$ S54

${ }^{13} \mathrm{C}$ NMR of compound $\mathbf{5 4}\left(\mathrm{CDCl}_{3}, 151 \mathrm{MHz}\right) \quad$ S54

${ }^{1} \mathrm{H}$ NMR of compound $\mathbf{5 5 a}\left(\mathrm{CDCl}_{3}, 600 \mathrm{MHz}\right) \quad$ S55

${ }^{13} \mathrm{C}$ NMR of compound 55a $\left(\mathrm{CDCl}_{3}, 151 \mathrm{MHz}\right) \quad \mathrm{S} 55$

Experimental procedures and physical data for compounds: $\quad$ S55

$16 \alpha, 16 \beta \quad$ S56

$17 \quad$ S56

18 S57

19 S57

20 S57

21a $\quad$ S58

23a 2 S58

23b $\quad$ S59

25A $\quad$ S59

25B $\quad$ S60

25C

26 S60

$27 \quad$ S61

30 S61

$31 \quad$ S61

$32 \quad$ S62

33a $\quad$ S62

34a $\quad$ S62

33b $\quad$ S63

34b $\quad$ S63

$37 \quad$ S64

38a $\quad$ S64

38b $\quad$ S64

39a $\quad$ S65

39b $\quad$ S65

40a $\quad$ S66

41a $\quad$ S66

$43 \quad$ S67

$44 \quad$ S67

$45 \quad$ S67

46a $\quad$ S68

$49 \quad$ S68

50 S69

512 S69

52 S69

$53 \quad$ S70

$54 \quad$ S70 


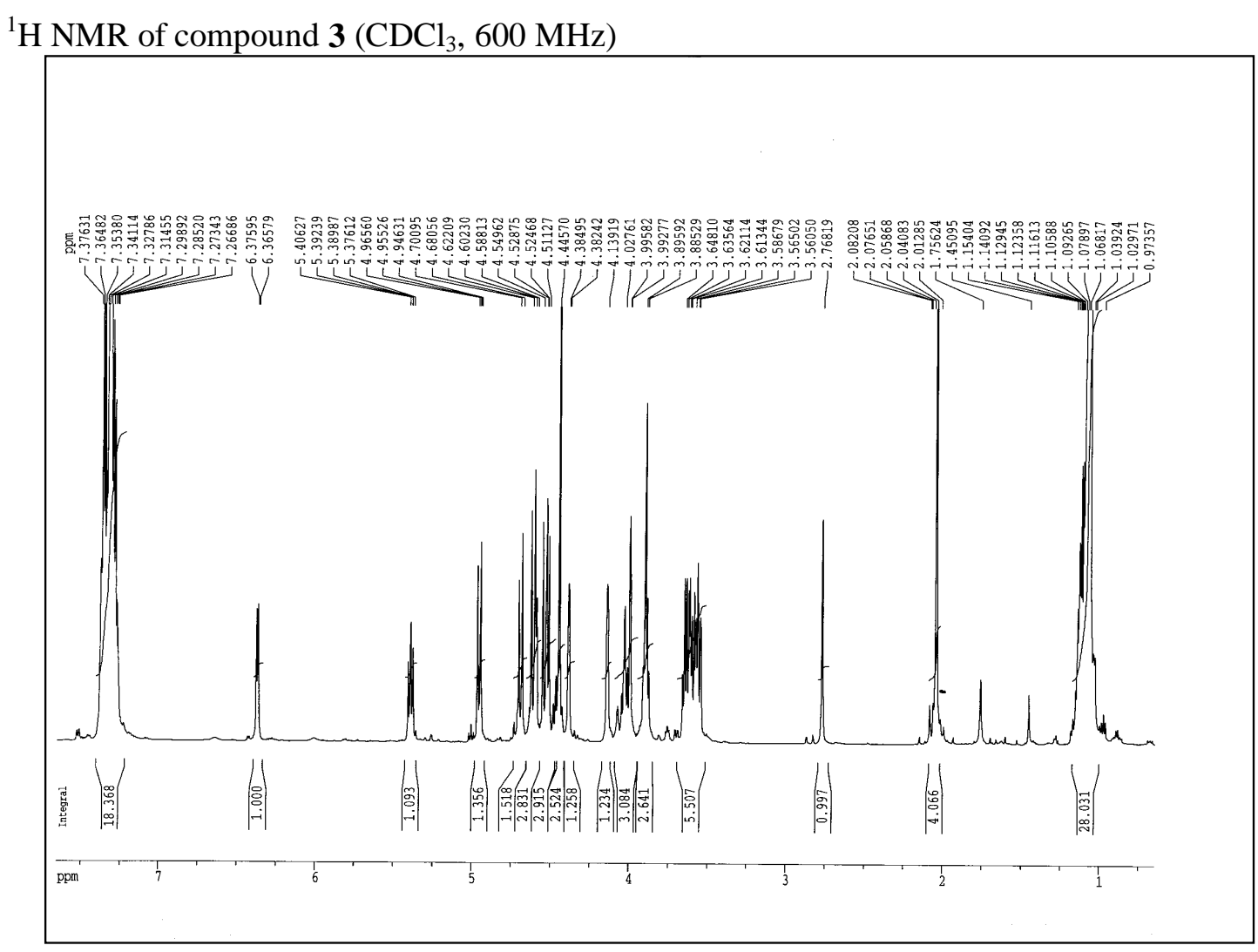

${ }^{13} \mathrm{C} \mathrm{NMR}$ of compound $\mathbf{3}\left(\mathrm{CDCl}_{3}, 151 \mathrm{MHz}\right)$

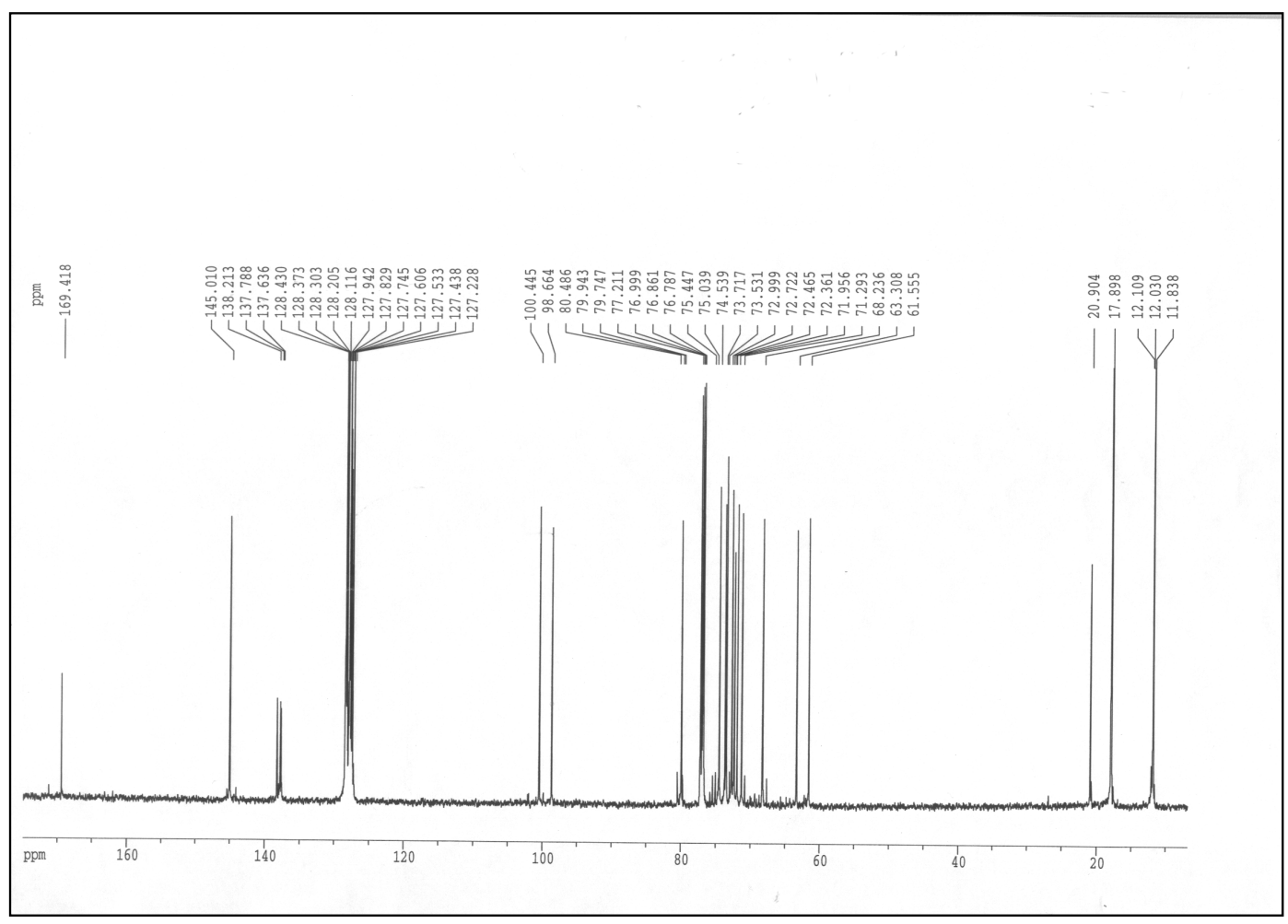




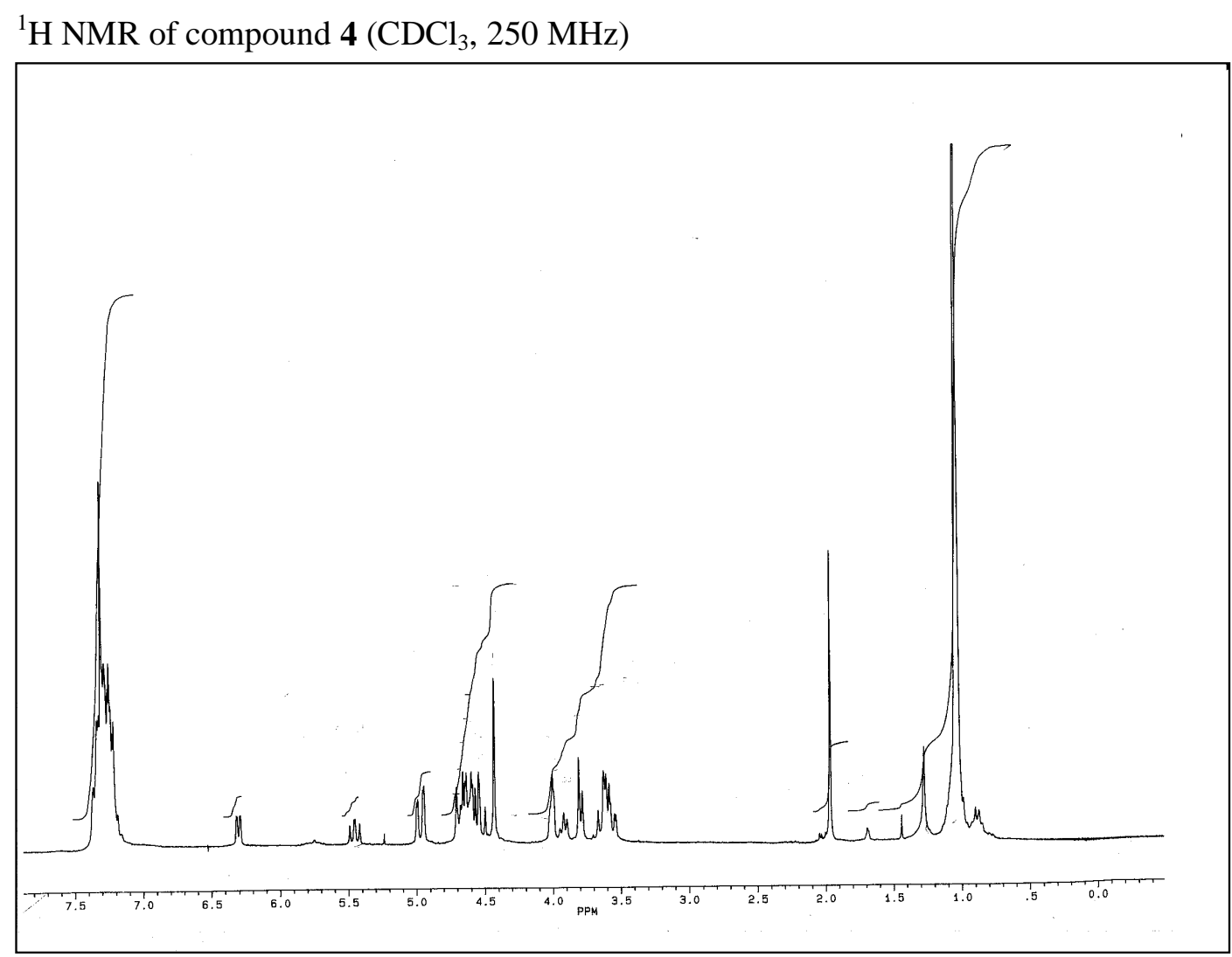

${ }^{1} \mathrm{H}$ NMR of compound $5\left(\mathrm{CDCl}_{3}, 250 \mathrm{MHz}\right)$

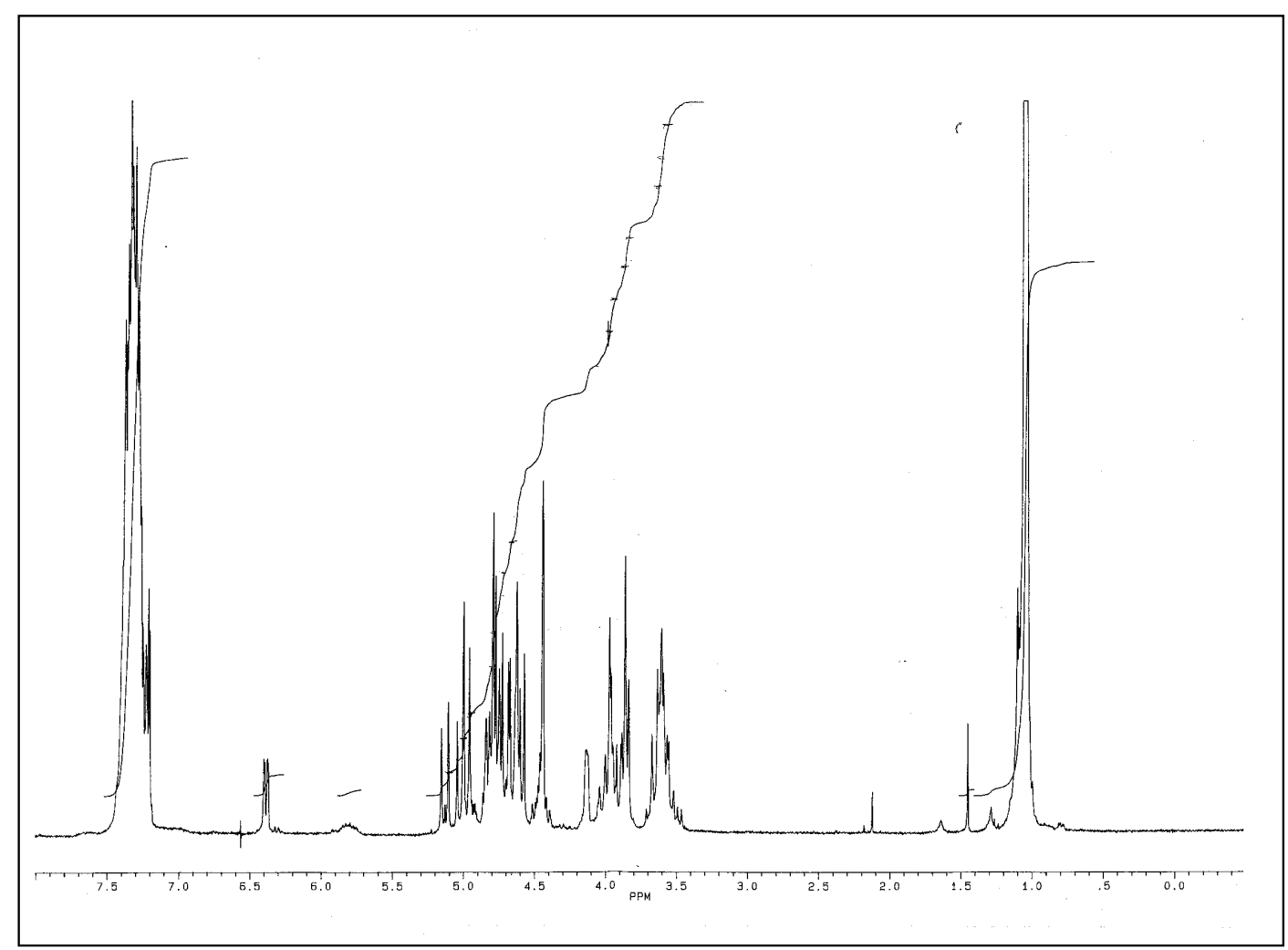


${ }^{1} \mathrm{H}$ NMR of compound $\mathbf{6}\left(\mathrm{CDCl}_{3}, 250 \mathrm{MHz}\right)$

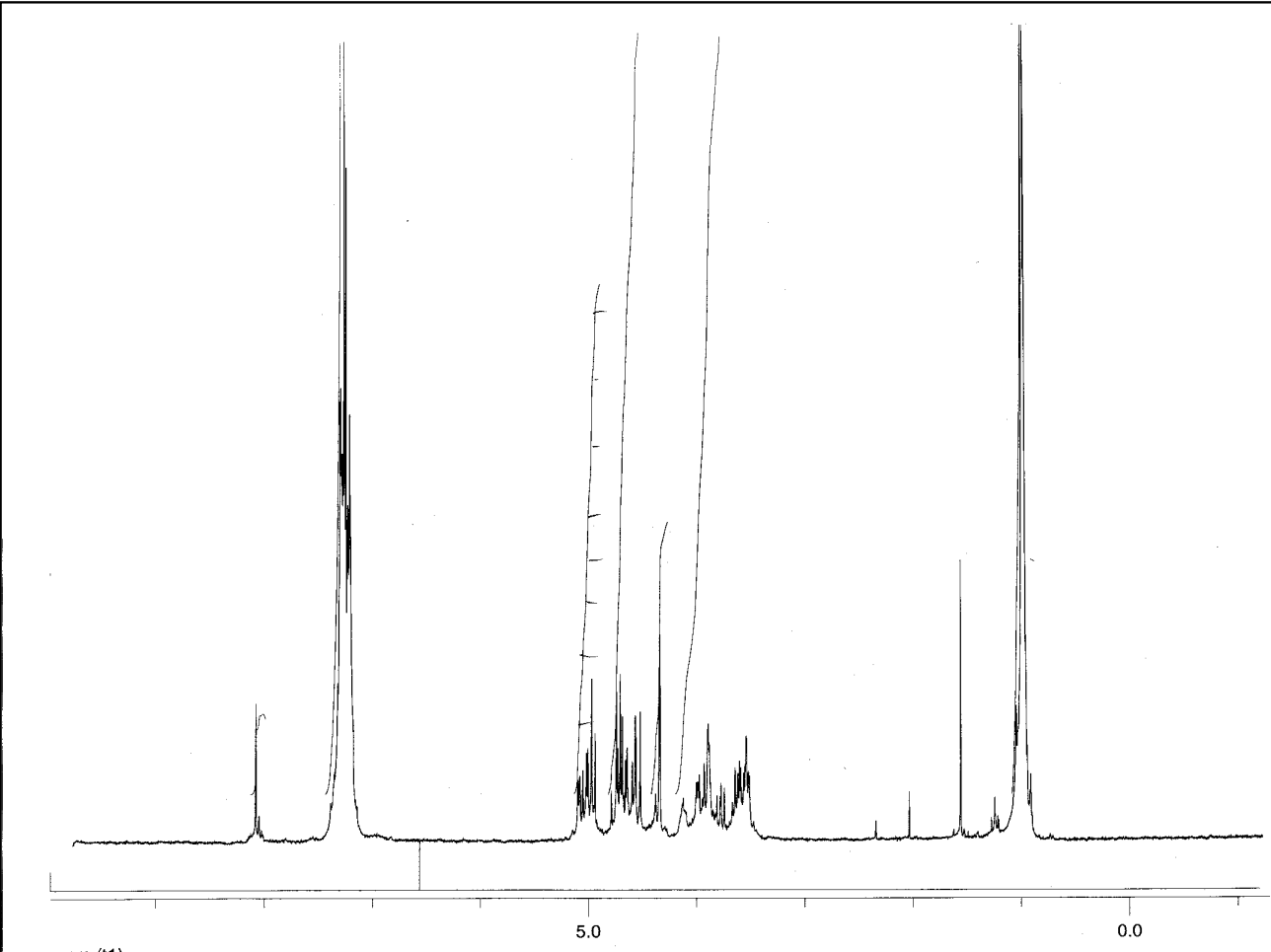

ppm (t1)

${ }^{1} \mathrm{H}$ NMR of compound $\mathbf{8 a}\left(\mathrm{CDCl}_{3}, 250 \mathrm{MHz}\right)$

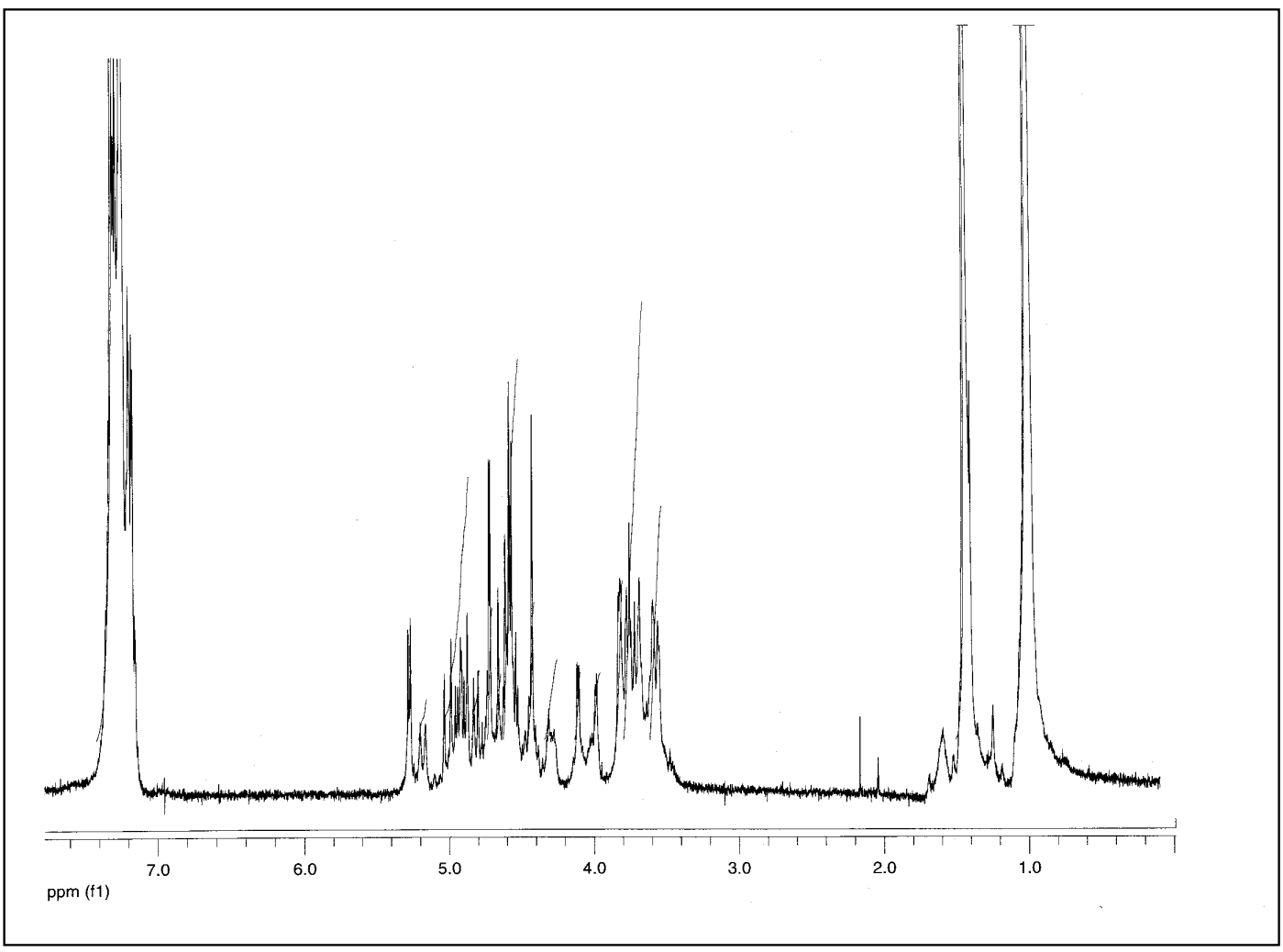


${ }^{13} \mathrm{C}$ NMR of compound $\mathbf{8 a}\left(\mathrm{CDCl}_{3}, 151 \mathrm{MHz}\right)$

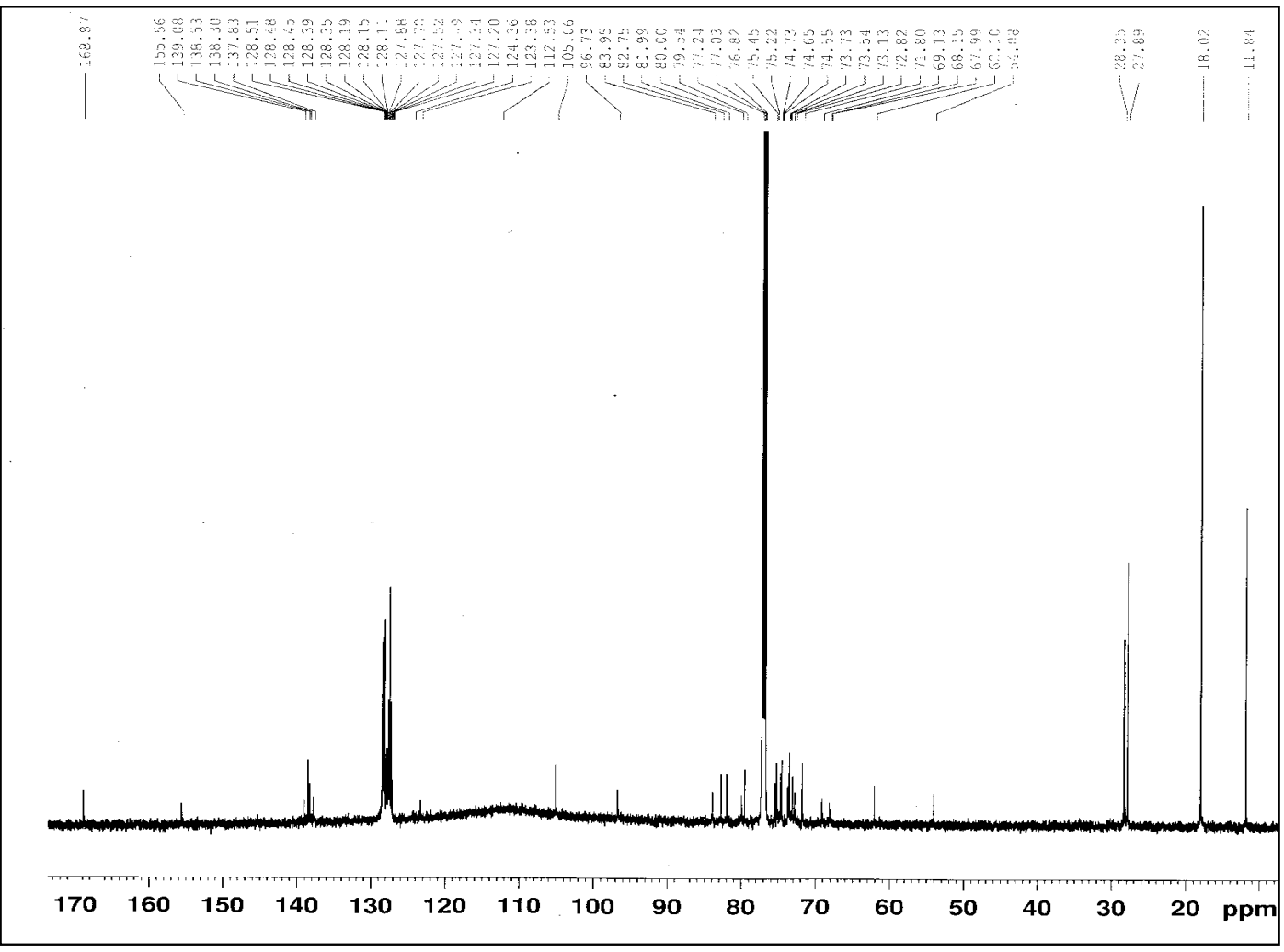

${ }^{1} \mathrm{H}$ NMR of compound $9 \mathbf{a}\left(\mathrm{CDCl}_{3}, 600 \mathrm{MHz}\right)$

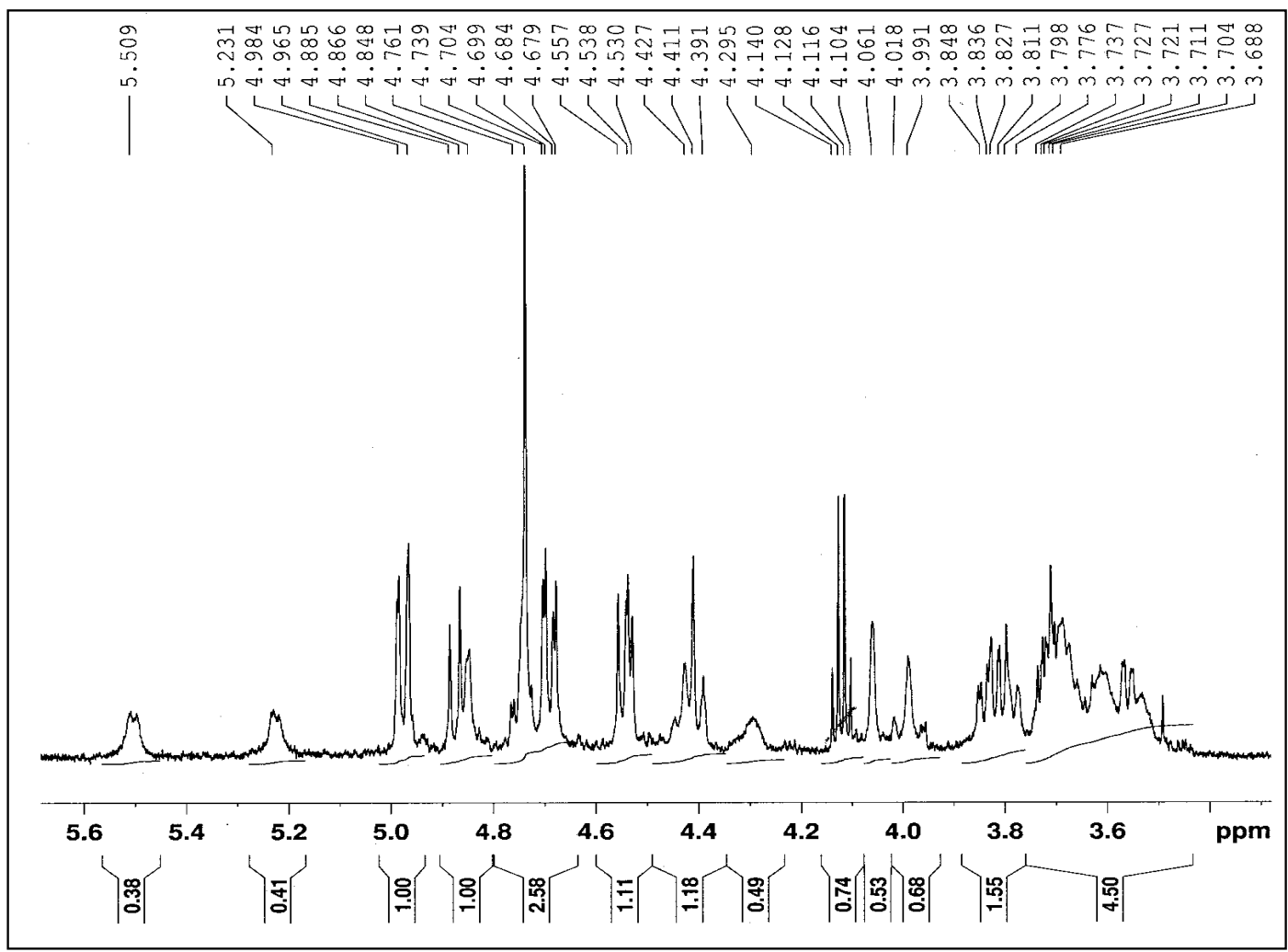


${ }^{1} \mathrm{H}$ NMR of compound $\mathbf{9 b}\left(\mathrm{CDCl}_{3}, 250 \mathrm{MHz}\right)$

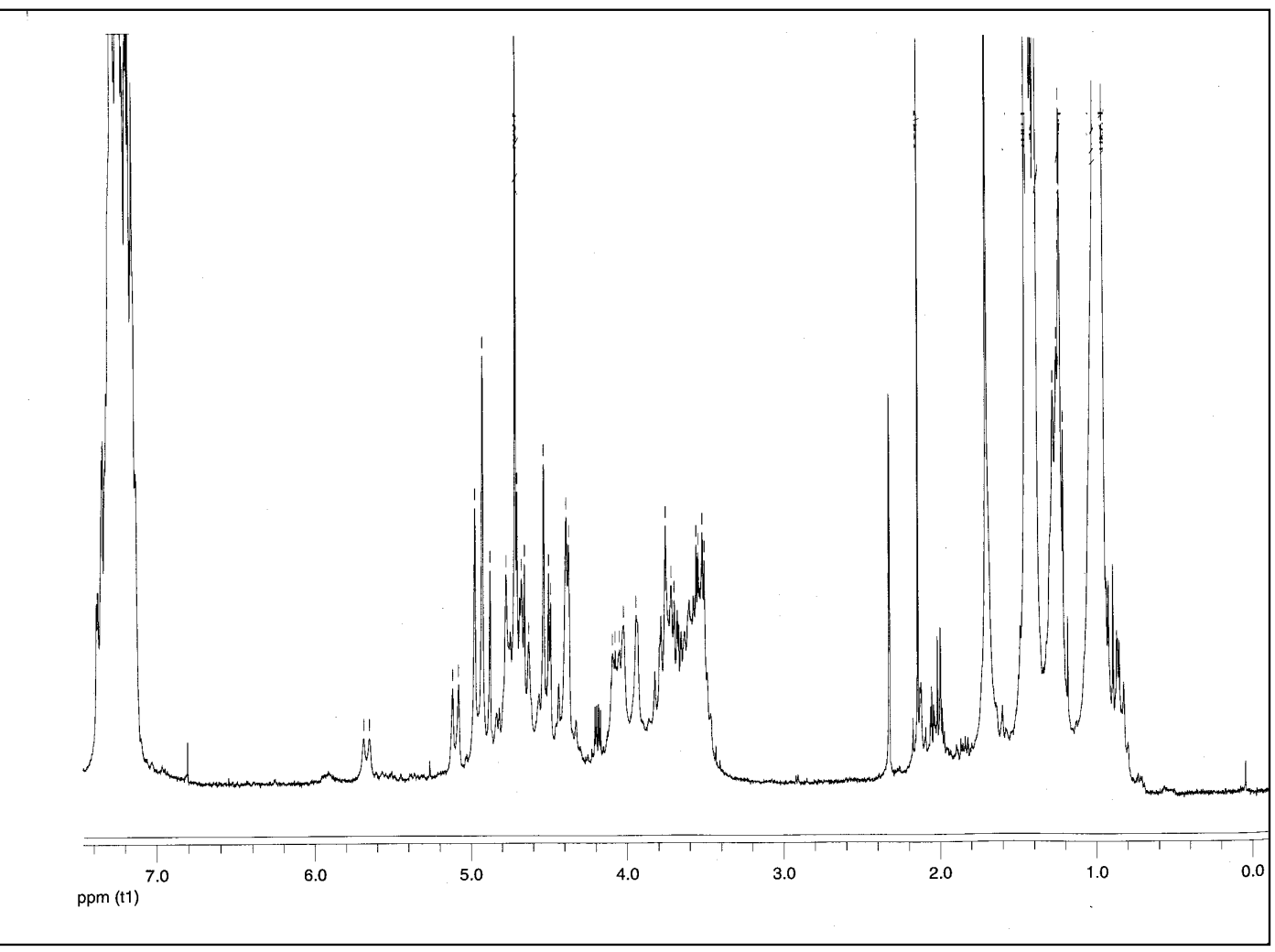

${ }^{13} \mathrm{C}$ NMR of compound $\mathbf{9 b}\left(\mathrm{CDCl}_{3}, 151 \mathrm{MHz}\right)$

020904

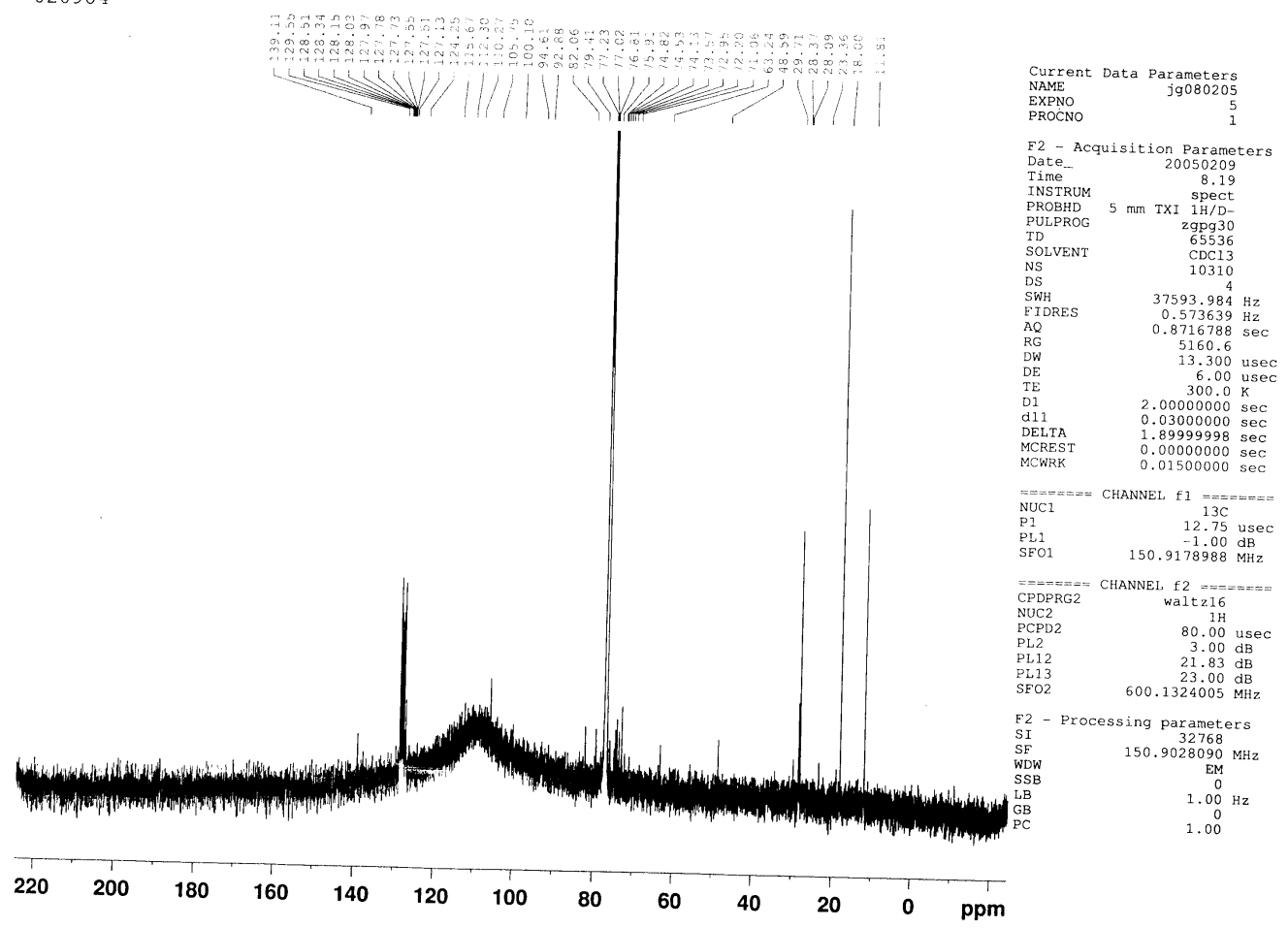


${ }^{1} \mathrm{H}$ NMR of compound $\mathbf{1 1}\left(\mathrm{CDCl}_{3}, 250 \mathrm{MHz}\right)$

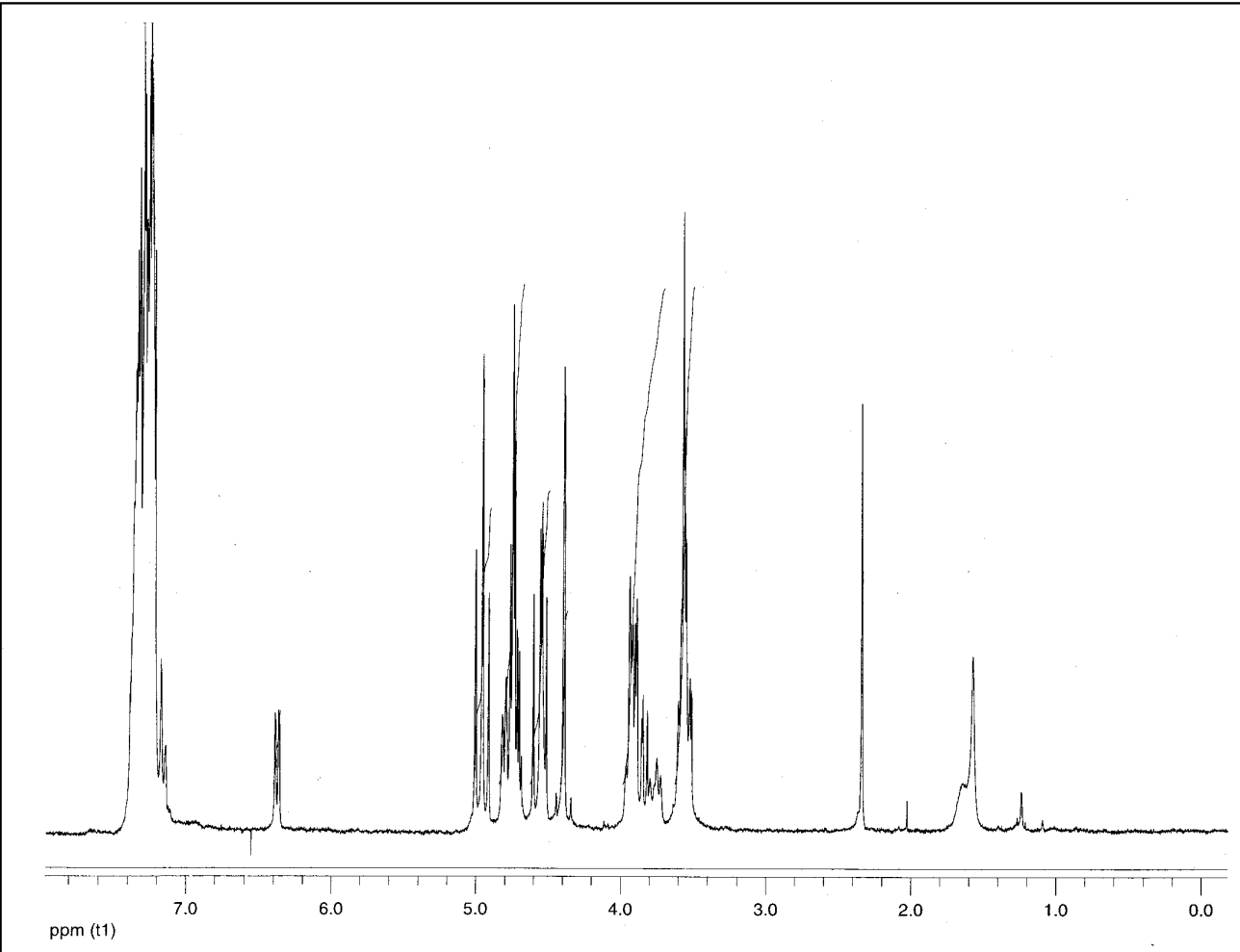

${ }^{13} \mathrm{C}$ NMR of compound $\mathbf{1 1}\left(\mathrm{CDCl}_{3}, 151 \mathrm{MHz}\right)$

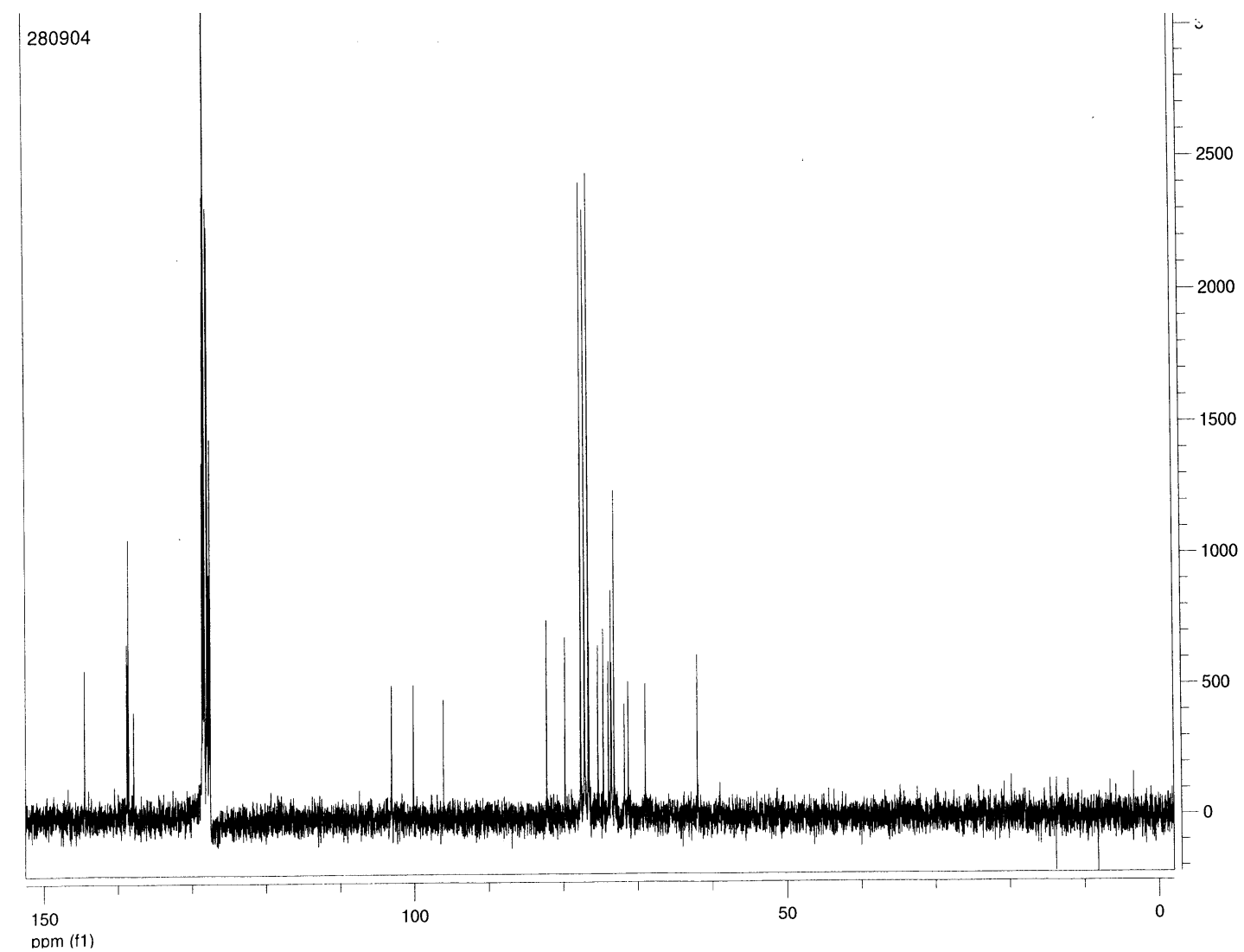


${ }^{1} \mathrm{H}$ NMR of compound $12\left(\mathrm{CDCl}_{3}, 600 \mathrm{MHz}\right)$

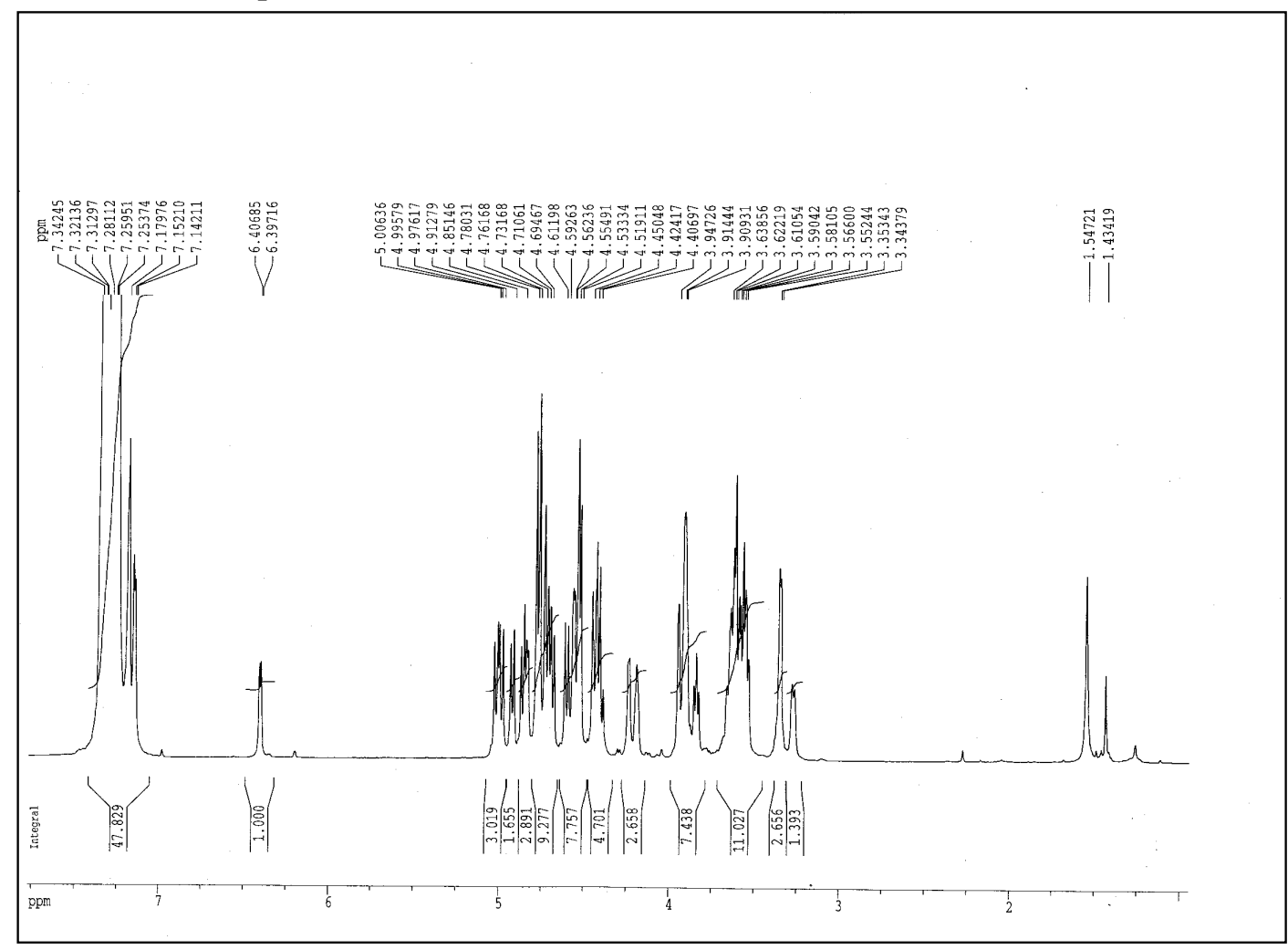

${ }^{13} \mathrm{C}$ NMR of compound $12\left(\mathrm{CDCl}_{3}, 151 \mathrm{MHz}\right)$

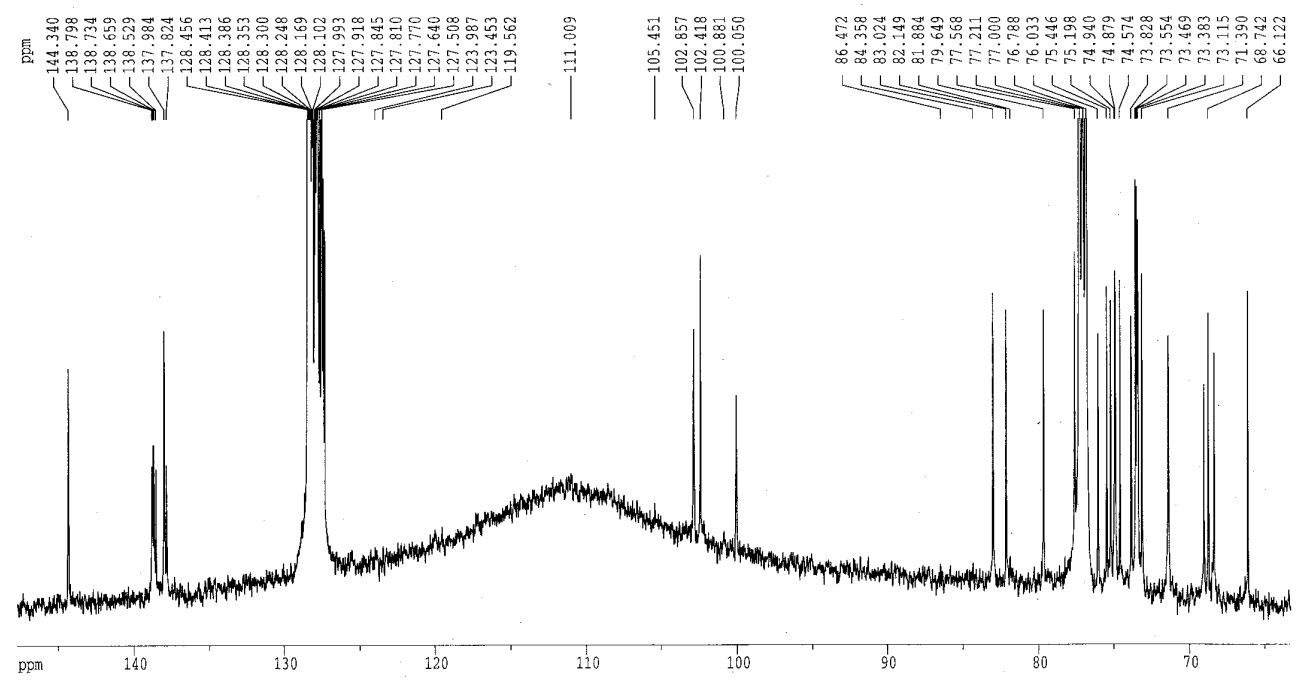


${ }^{1} \mathrm{H}$ NMR of compound $13\left(\mathrm{CDCl}_{3}, 600 \mathrm{MHz}\right)$

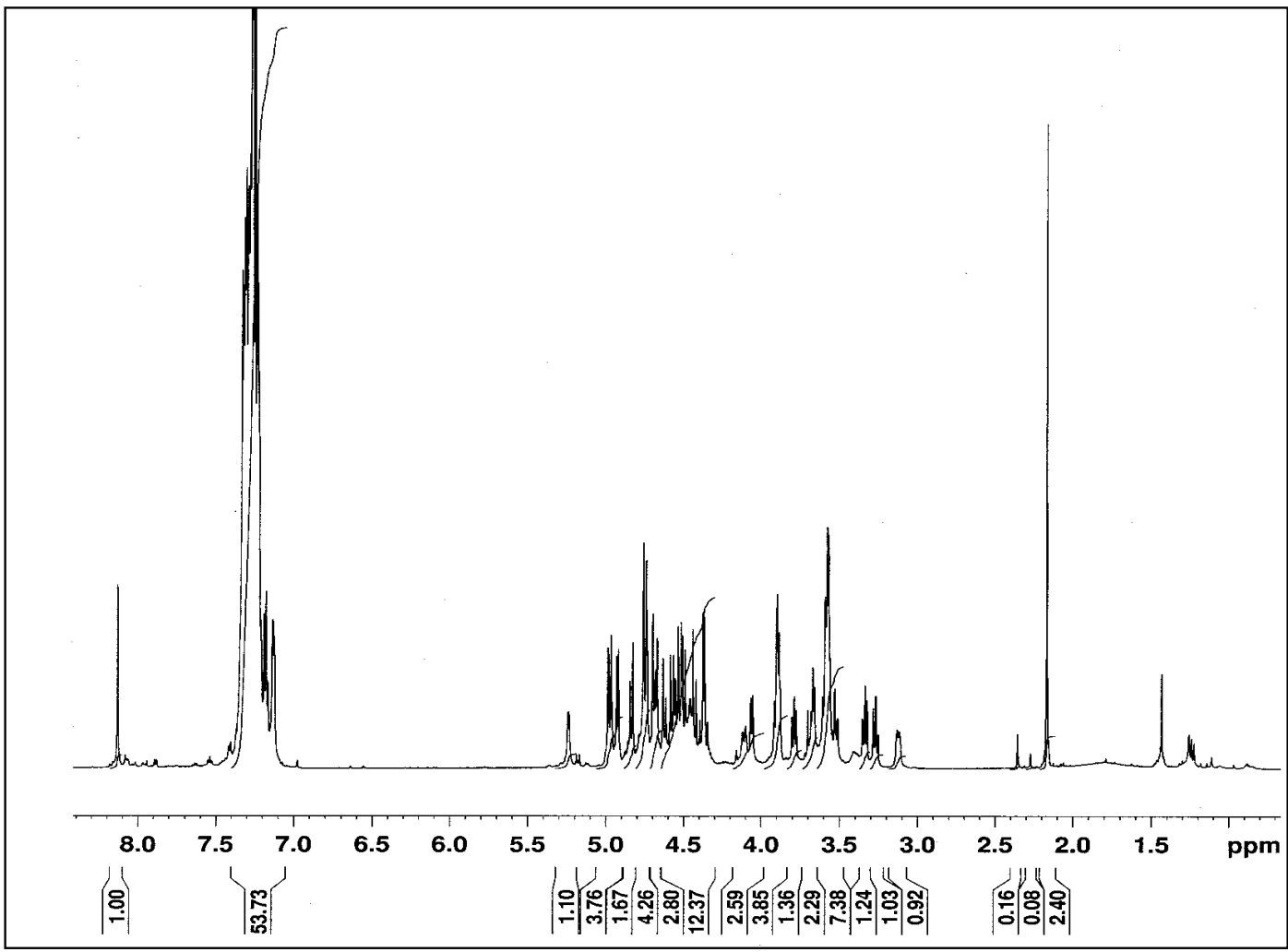

${ }^{13} \mathrm{C}$ NMR of compound $\mathbf{1 3}\left(\mathrm{CDCl}_{3}, 151 \mathrm{MHz}\right)$

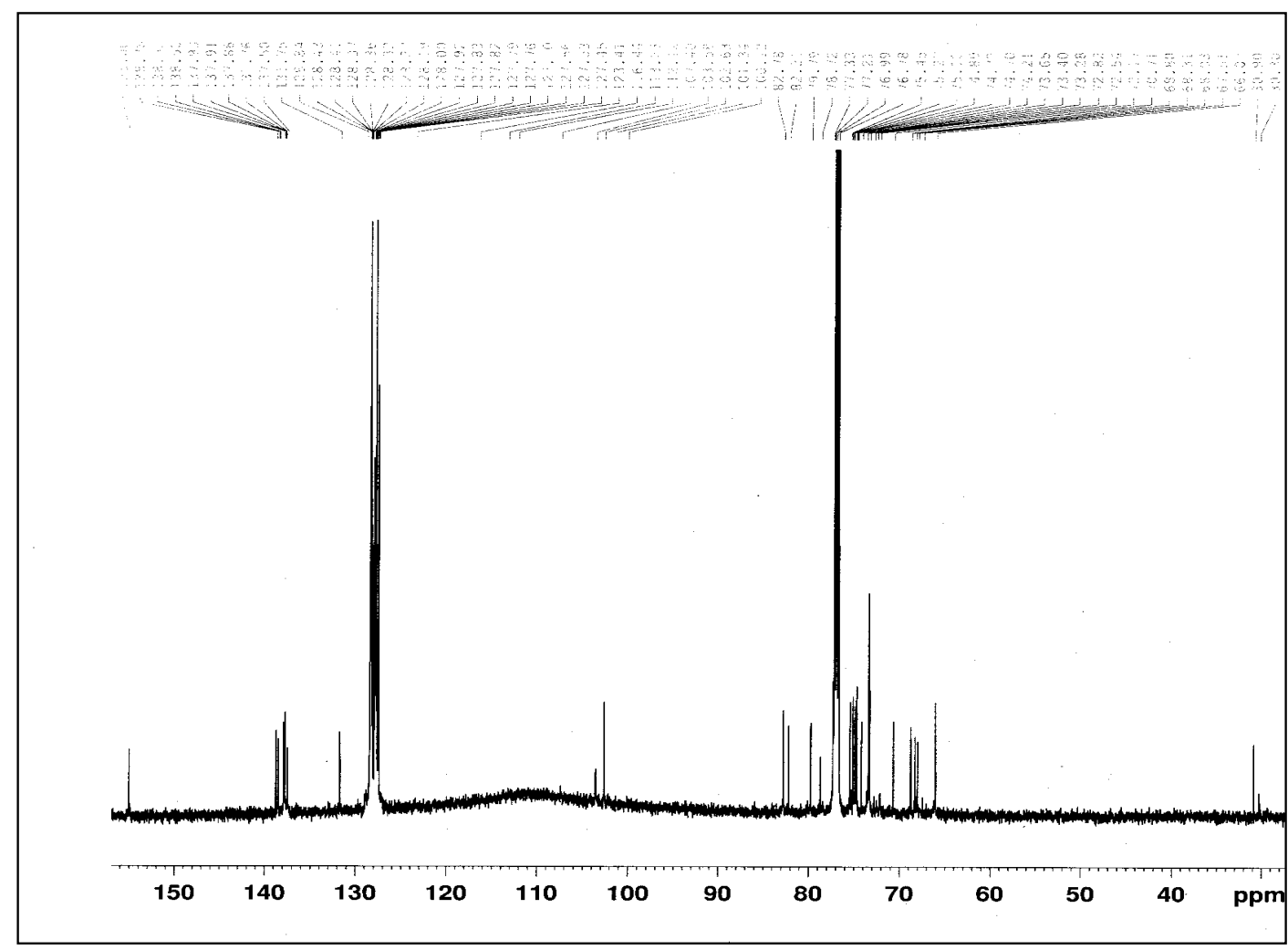




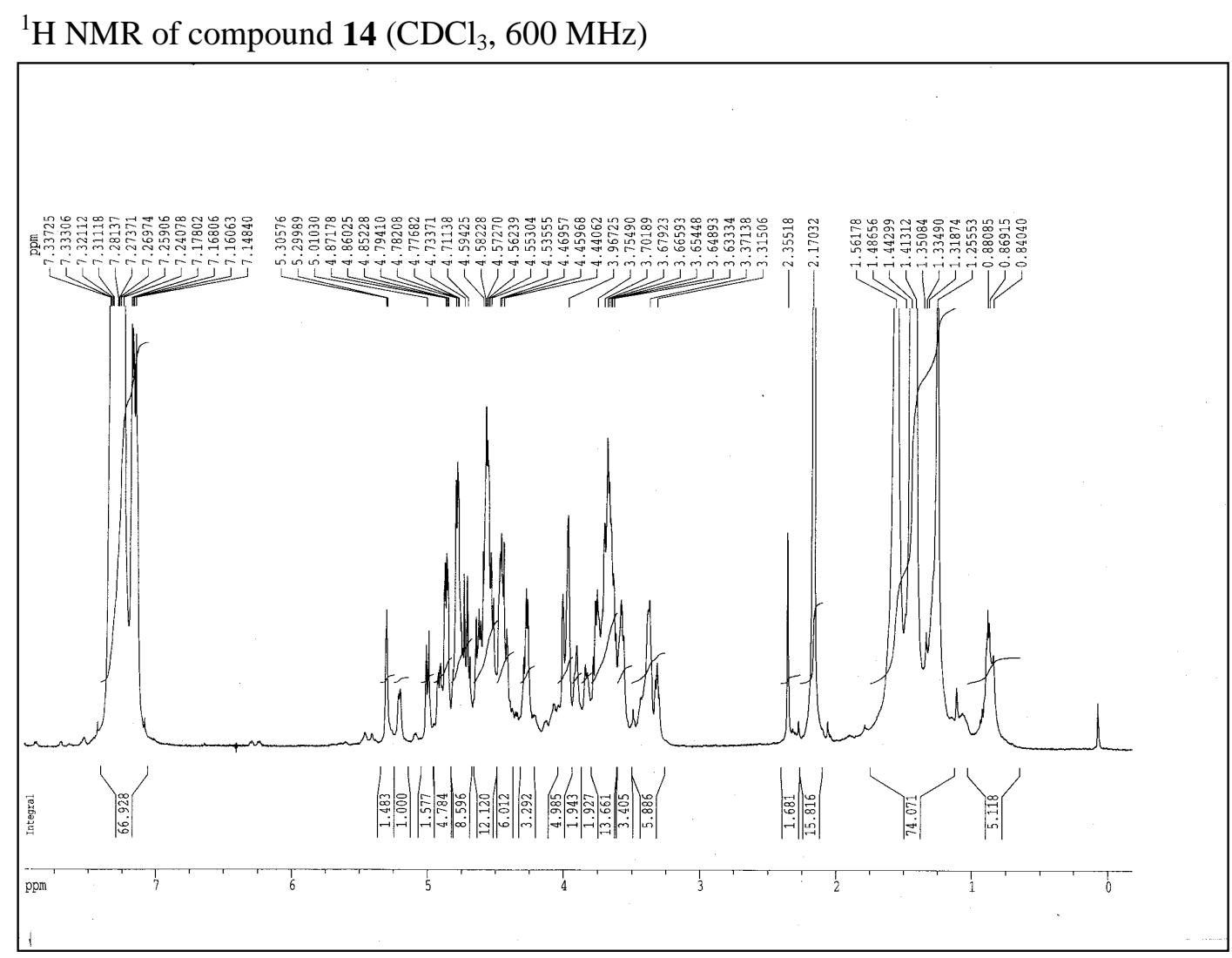

${ }^{1} \mathrm{H}$ NMR of compound $\mathbf{1 6} \alpha\left(\mathrm{CDCl}_{3}, 250 \mathrm{MHz}\right)$

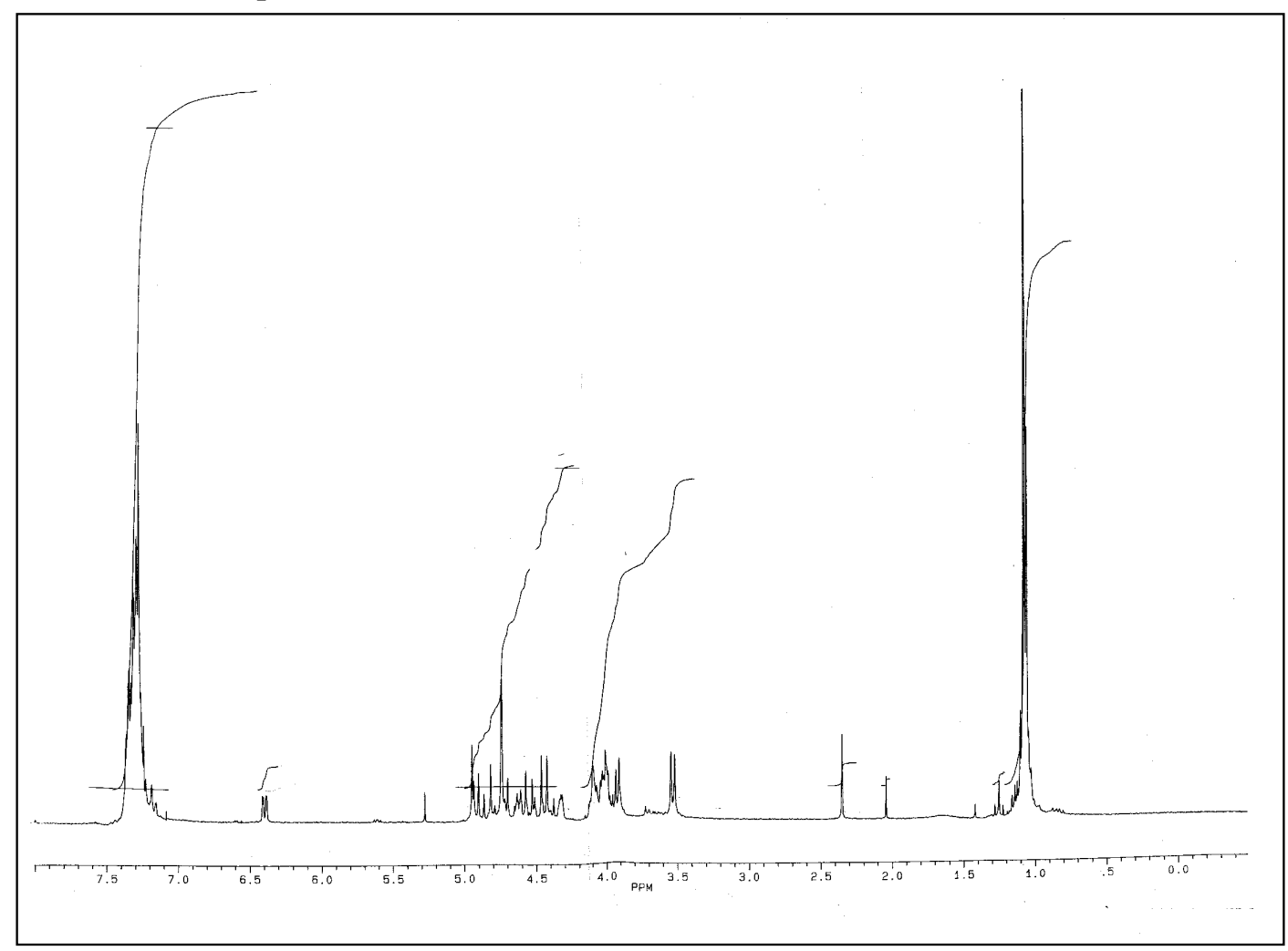


${ }^{13} \mathrm{C}$ NMR of compound $\mathbf{1 6} \alpha\left(\mathrm{CDCl}_{3}, 151 \mathrm{MHz}\right)$

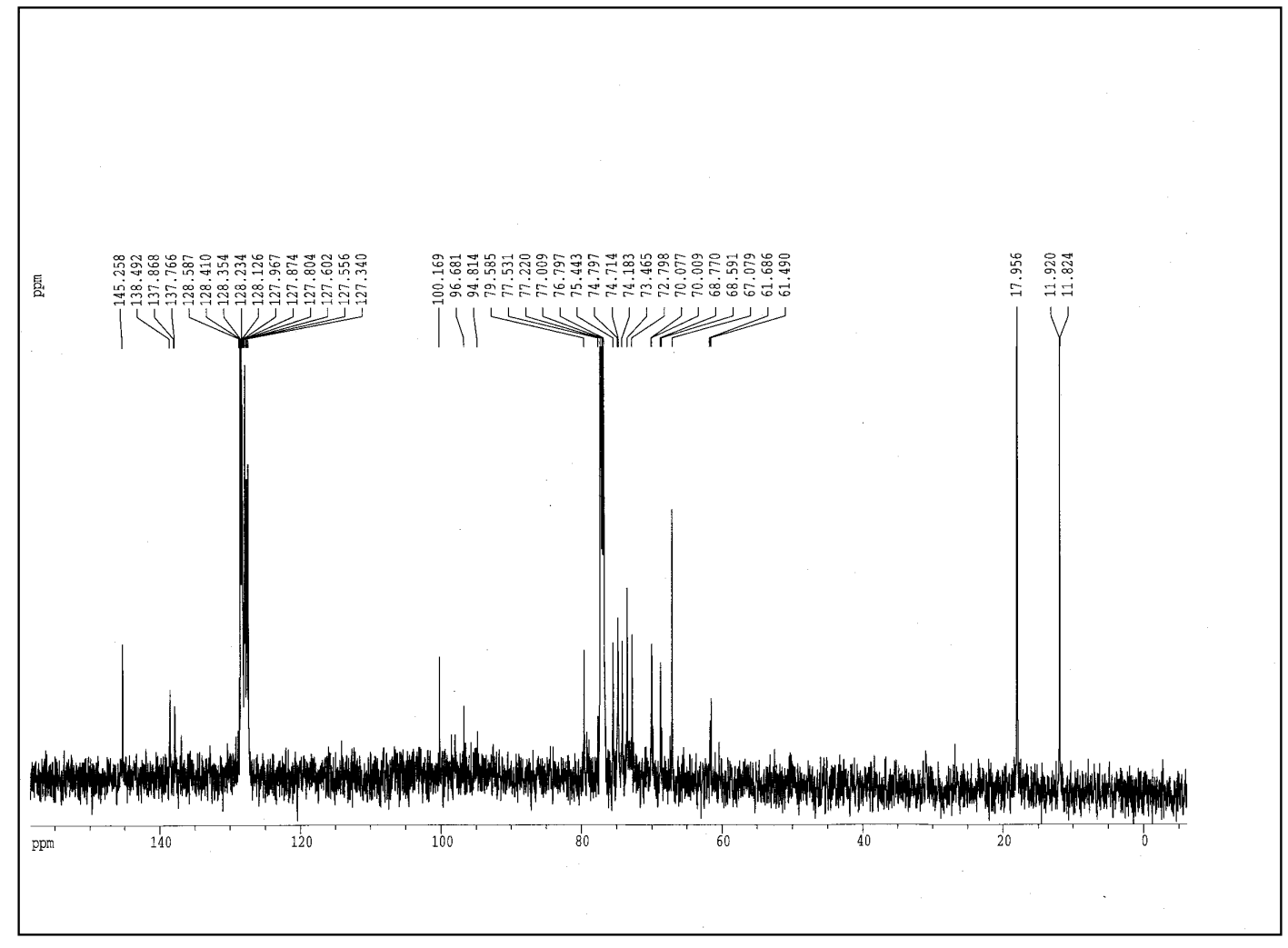

${ }^{1} \mathrm{H}$ NMR of compound $17\left(\mathrm{CDCl}_{3}, 600 \mathrm{MHz}\right)$

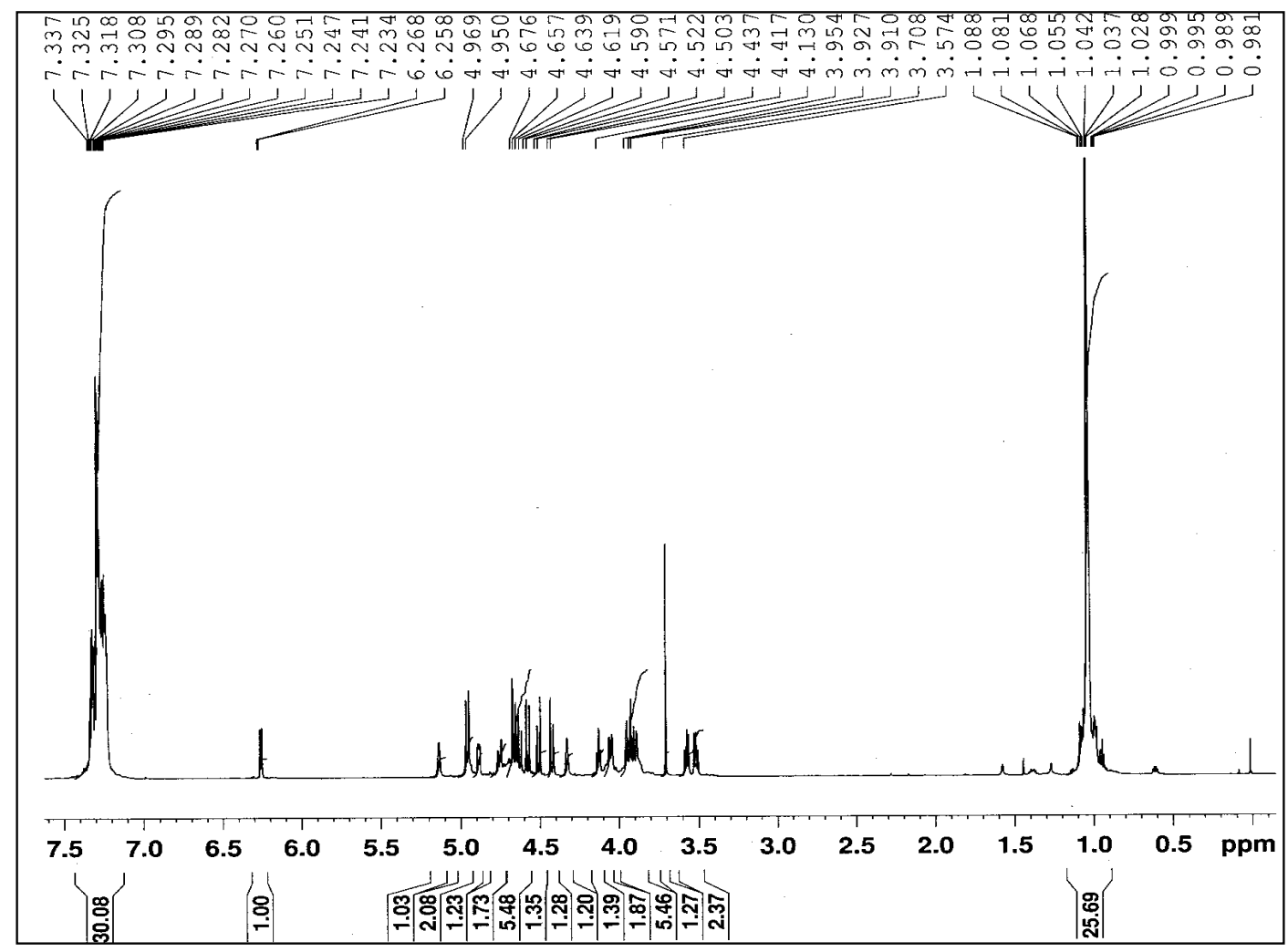


${ }^{13} \mathrm{C}$ NMR of compound $17\left(\mathrm{CDCl}_{3}, 151 \mathrm{MHz}\right)$

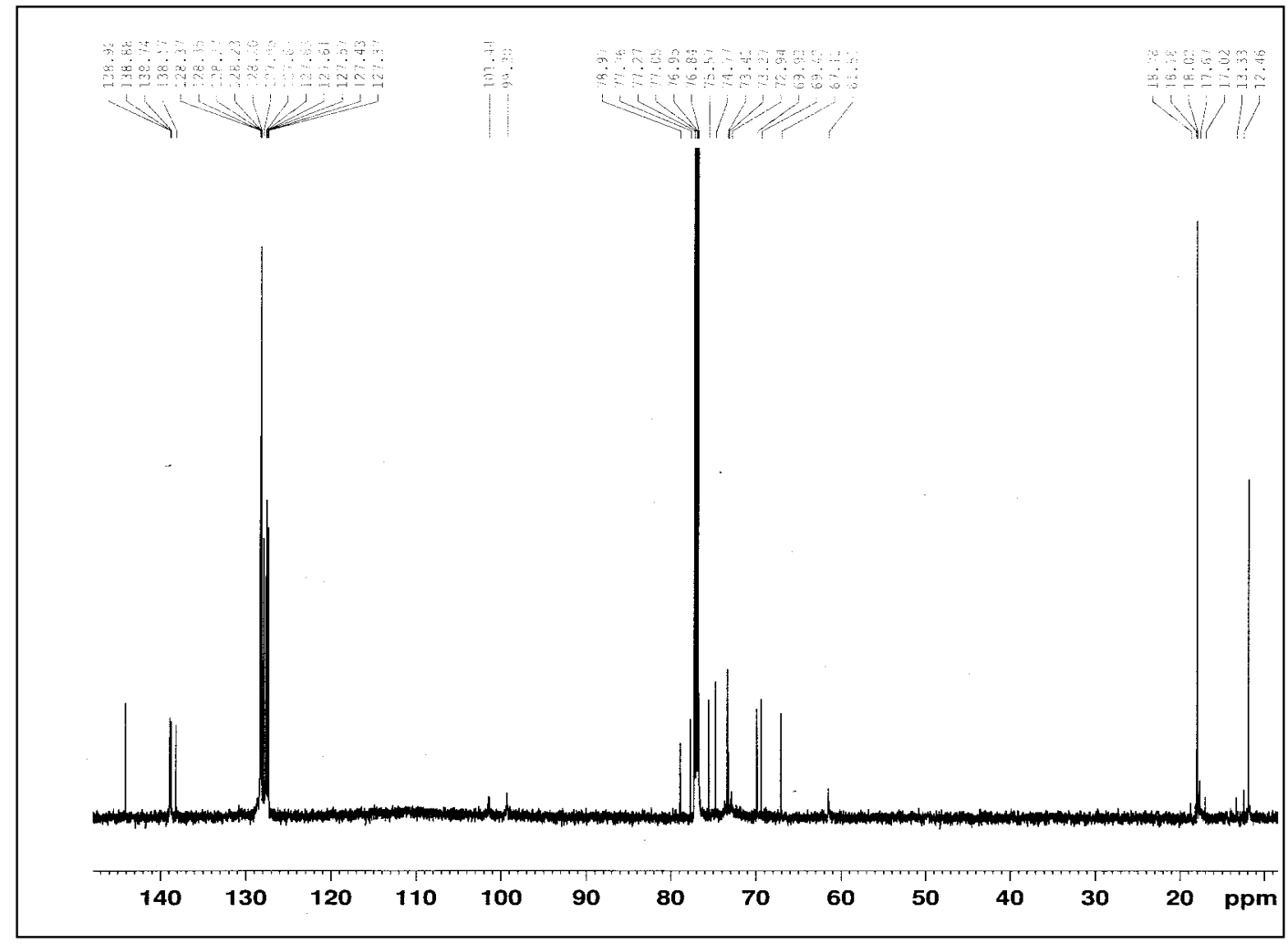

${ }^{1} \mathrm{H}$ NMR of compound $18\left(\mathrm{CDCl}_{3}, 250 \mathrm{MHz}\right)$

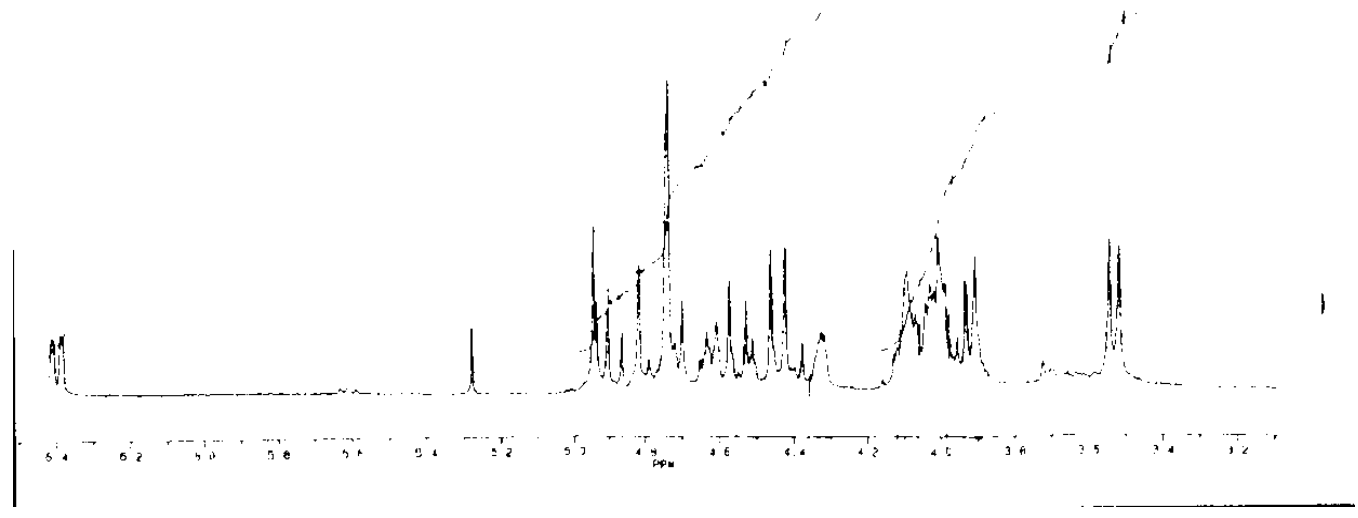


${ }^{13} \mathrm{C} \mathrm{NMR}$ of compound $18\left(\mathrm{CDCl}_{3}, 63 \mathrm{MHz}\right)$

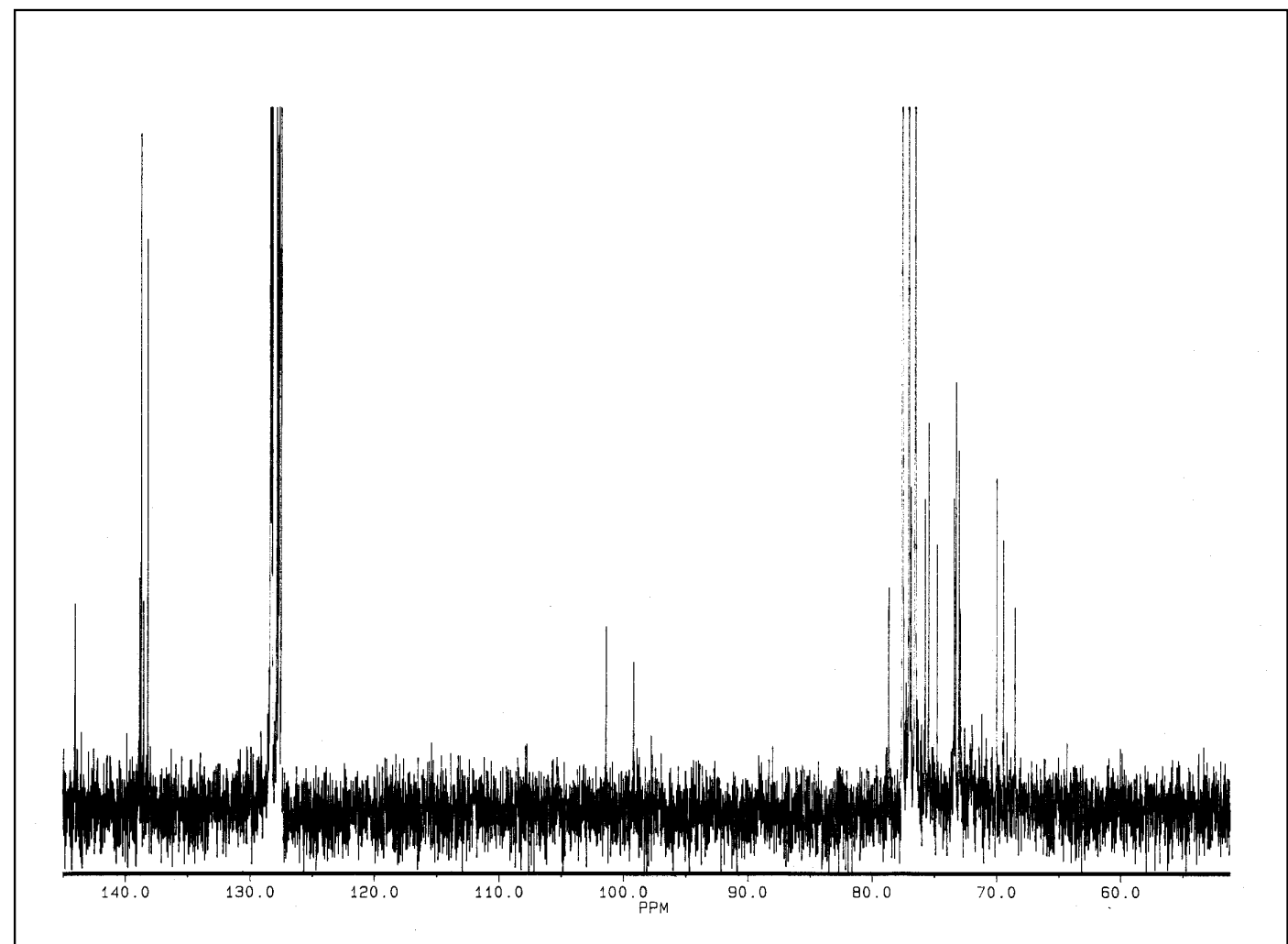

${ }^{1} \mathrm{H}$ NMR of compound $19\left(\mathrm{CDCl}_{3}, 250 \mathrm{MHz}\right)$

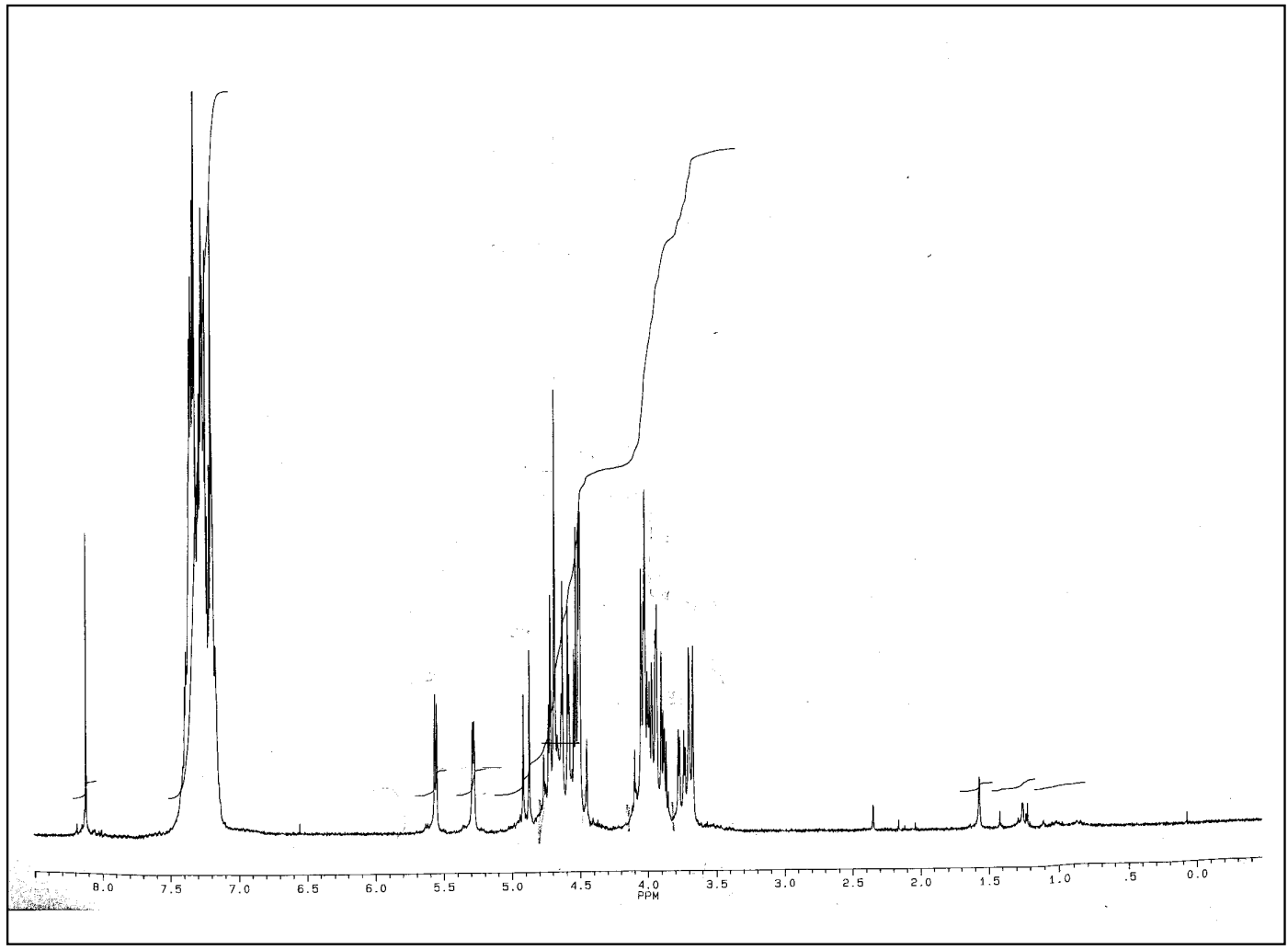


${ }^{13} \mathrm{C}$ NMR of compound $19\left(\mathrm{CDCl}_{3}, 63 \mathrm{MHz}\right)$

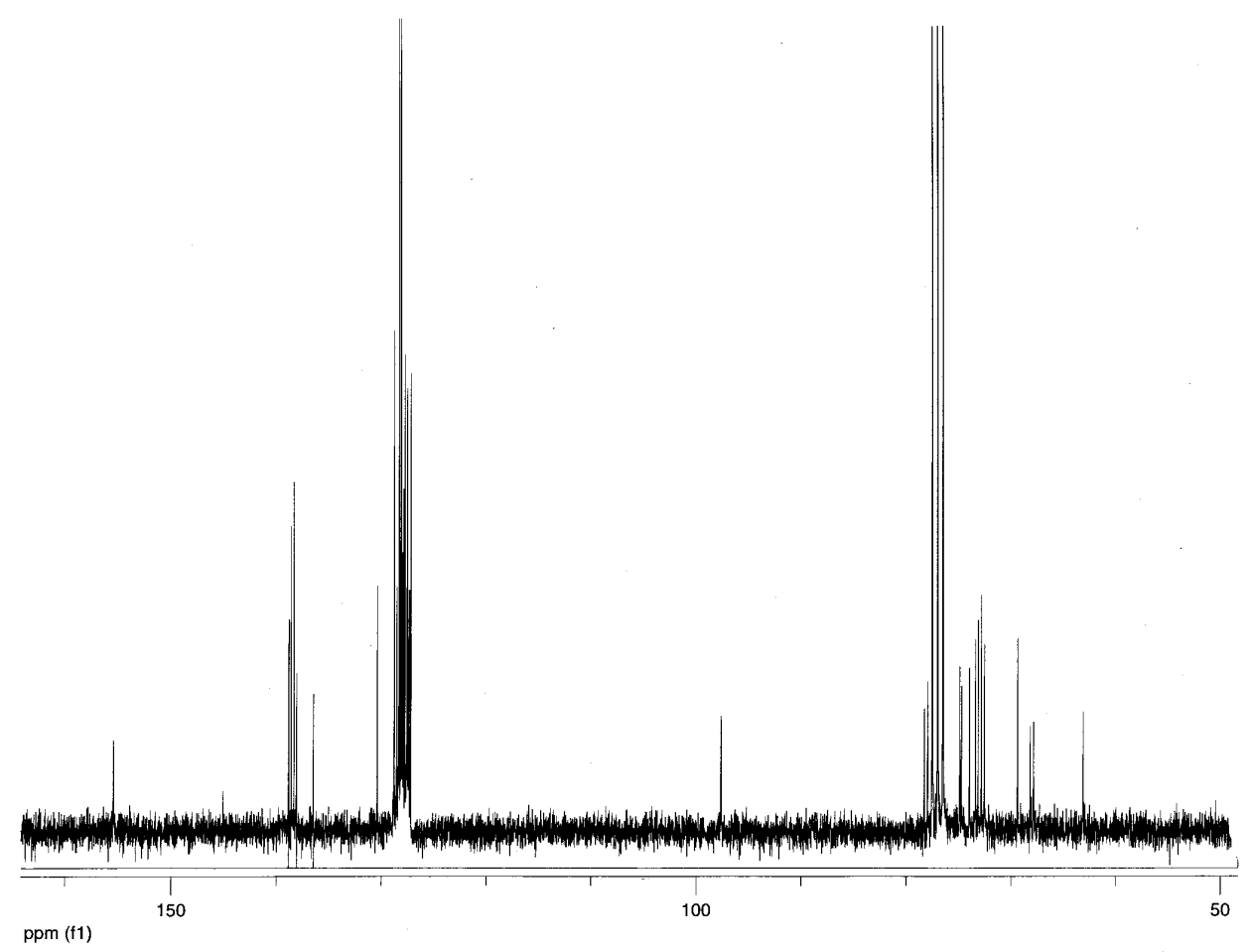

${ }^{1} \mathrm{H}$ NMR of compound $20\left(\mathrm{CDCl}_{3}, 250 \mathrm{MHz}\right)$

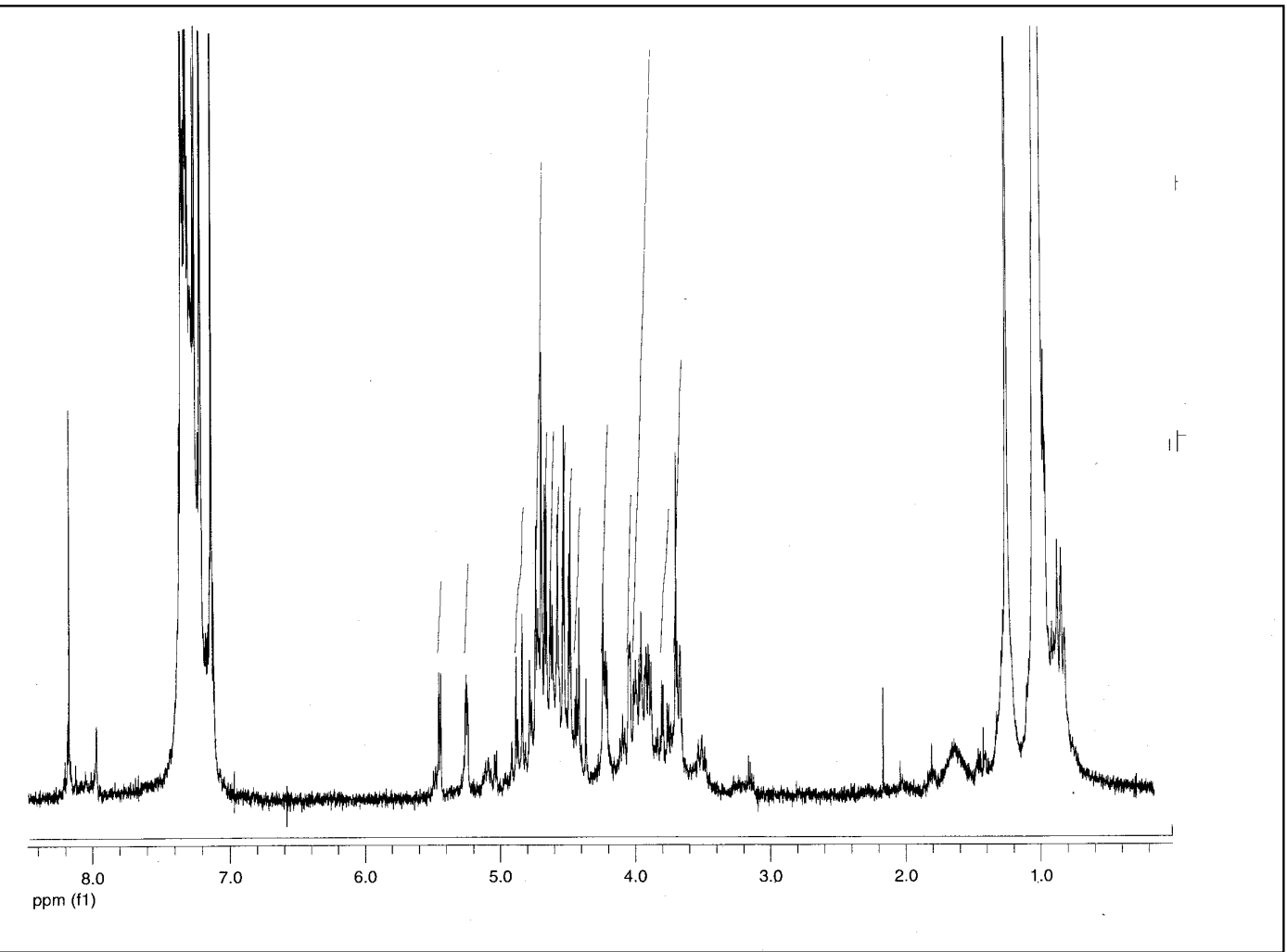


${ }^{1} \mathrm{H}$ NMR of compound $21 \mathrm{a}\left(\mathrm{CDCl}_{3}, 600 \mathrm{MHz}\right)$

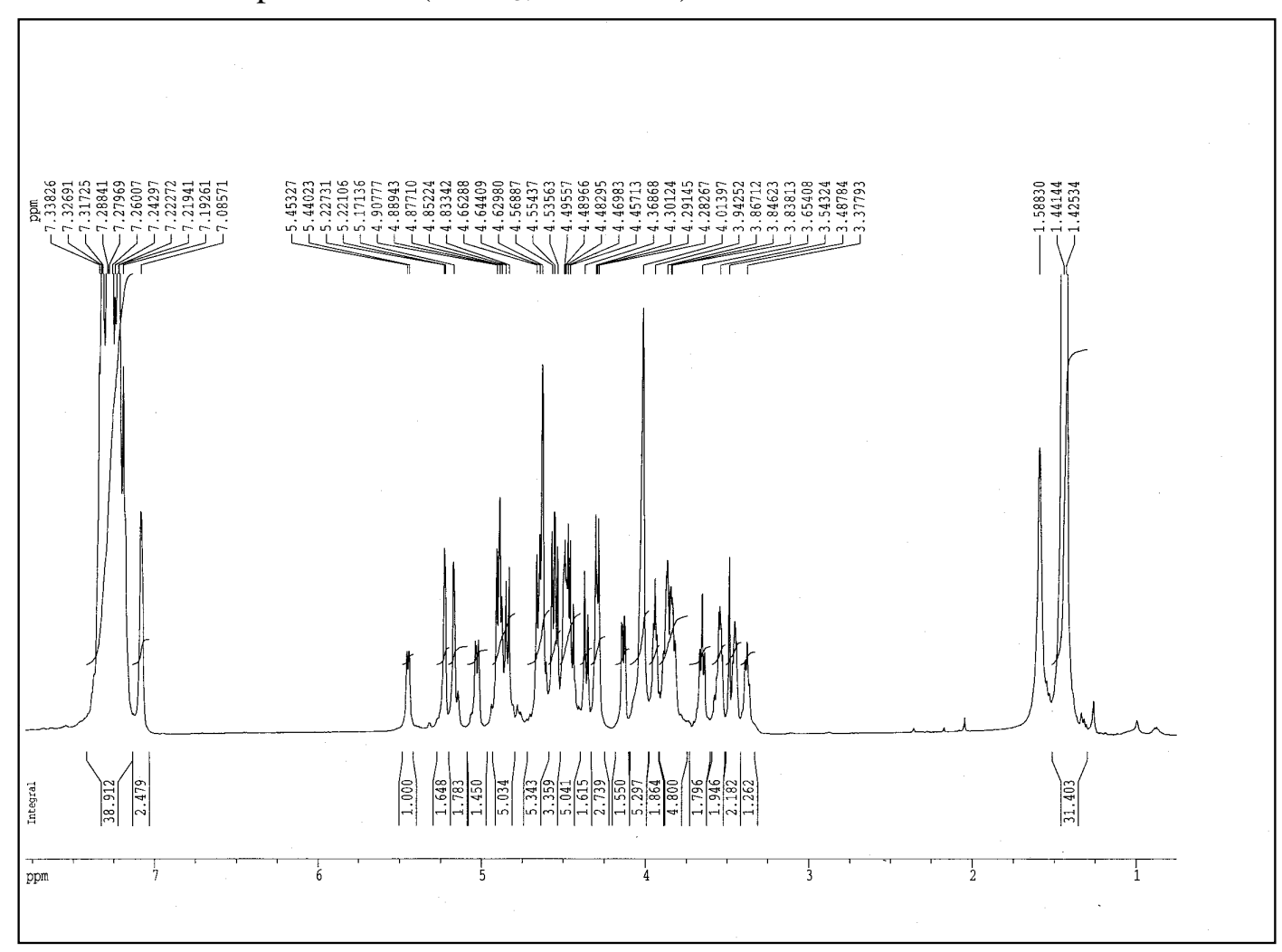

${ }^{13} \mathrm{C}$ NMR of compound $21 \mathbf{a}\left(\mathrm{CDCl}_{3}, 151 \mathrm{MHz}\right)$

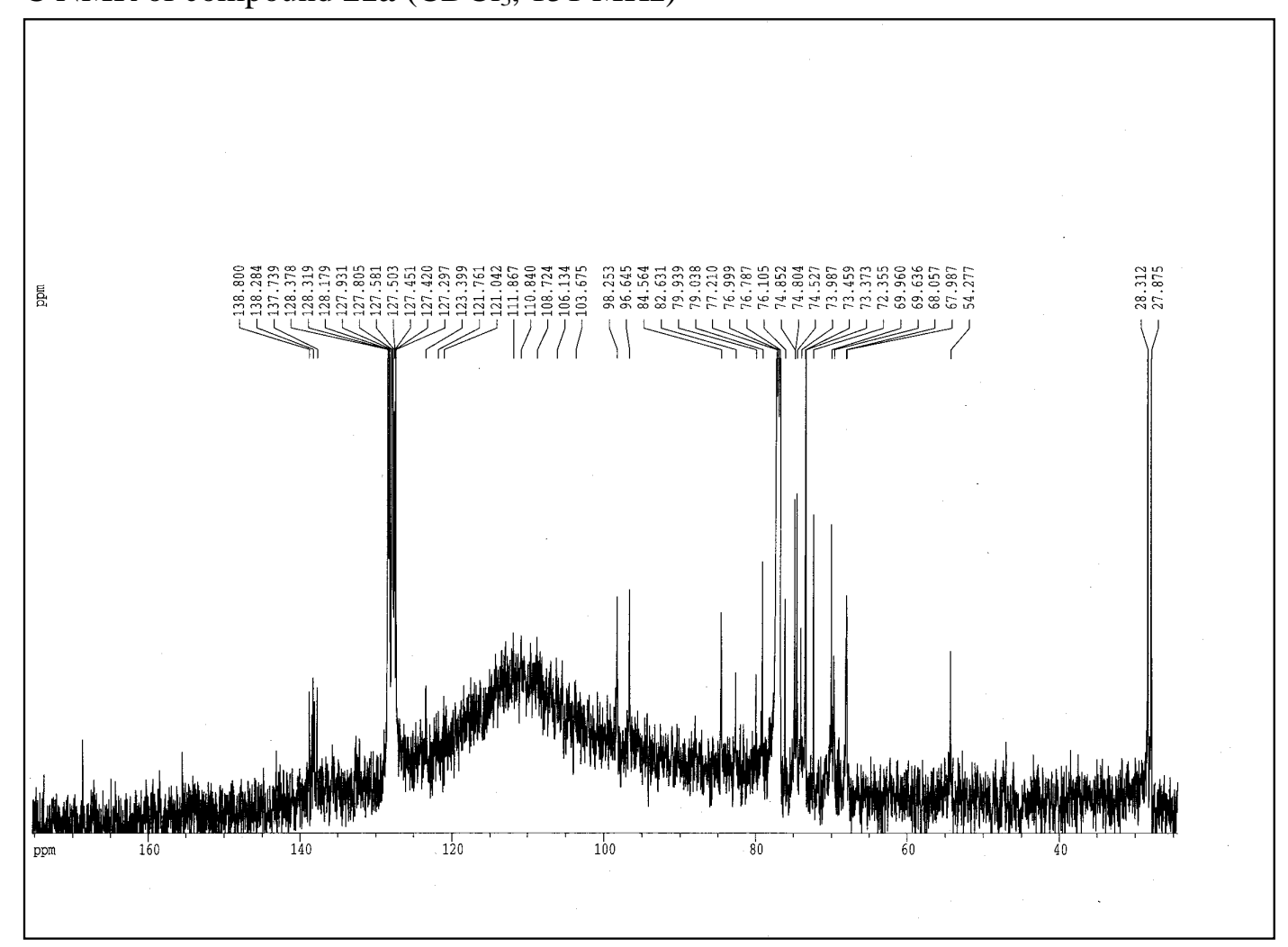


${ }^{1} \mathrm{H}$ NMR of compound 22a $\left(\mathrm{CDCl}_{3}, 600 \mathrm{MHz}\right)$

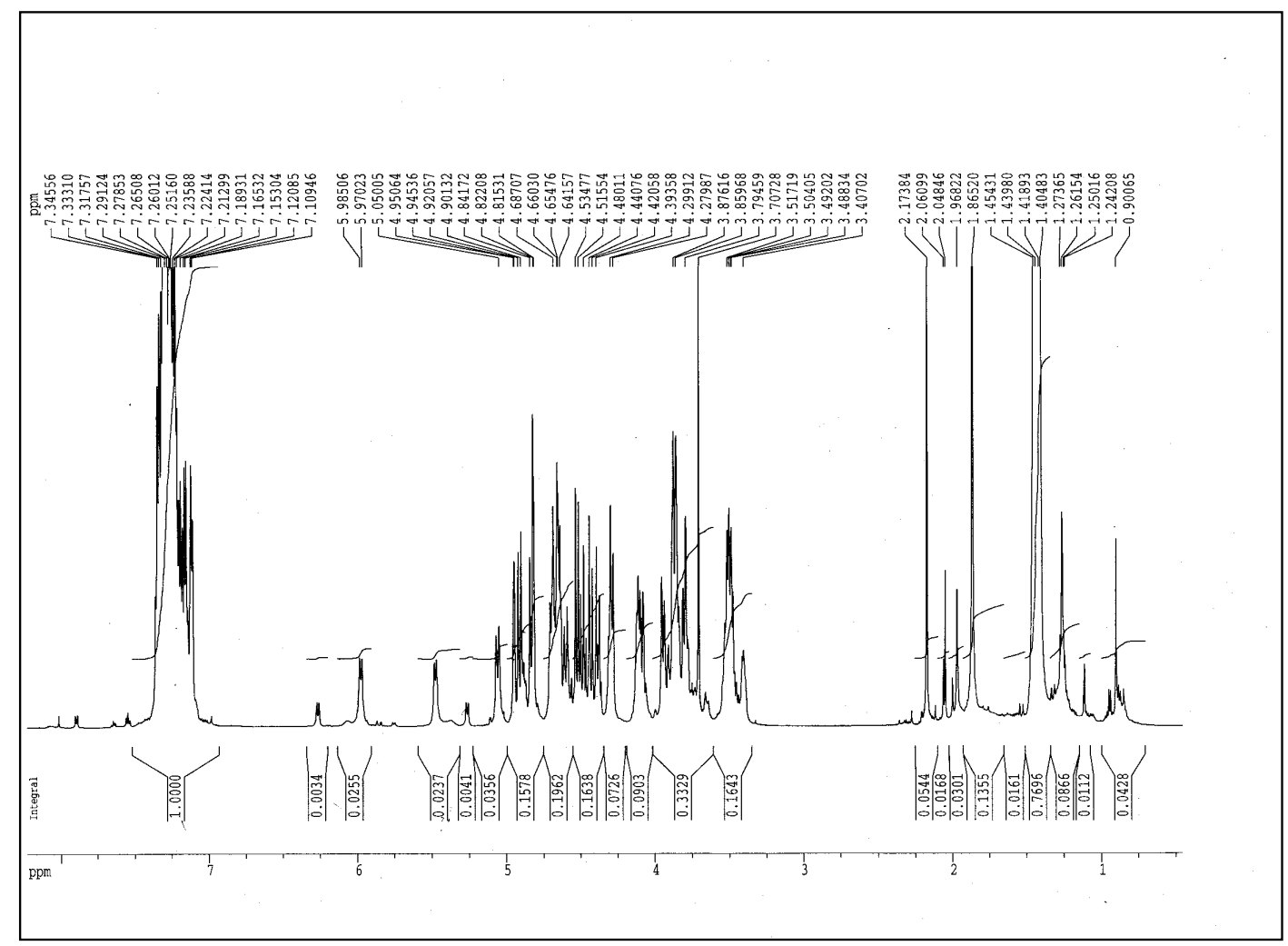

${ }^{13} \mathrm{C}$ NMR of compound $22 \mathrm{a}\left(\mathrm{CDCl}_{3}, 151 \mathrm{MHz}\right)$ 


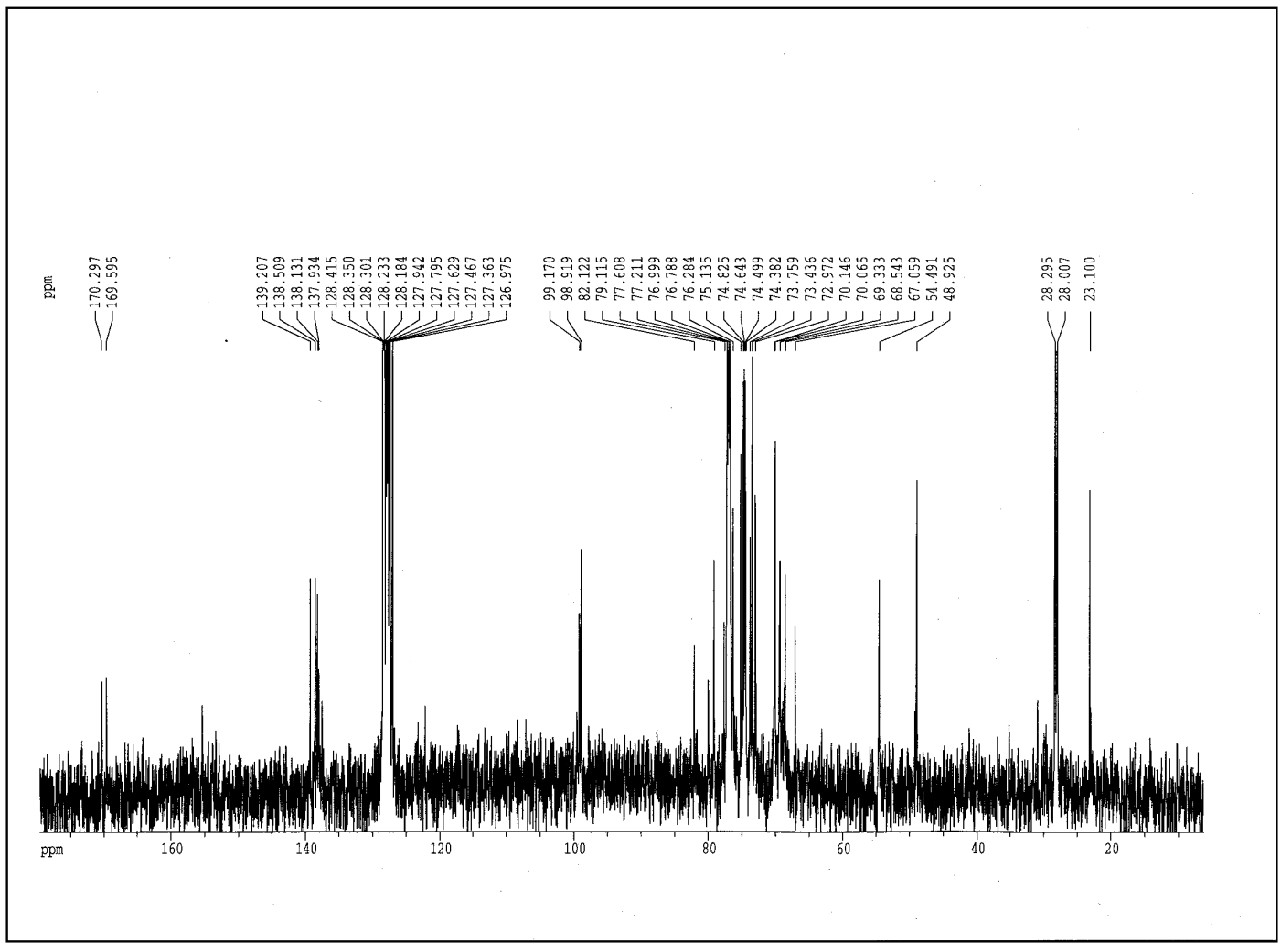

${ }^{1} \mathrm{H}$ NMR of compound 23a $\left(\mathrm{CDCl}_{3}, 250 \mathrm{MHz}\right)$

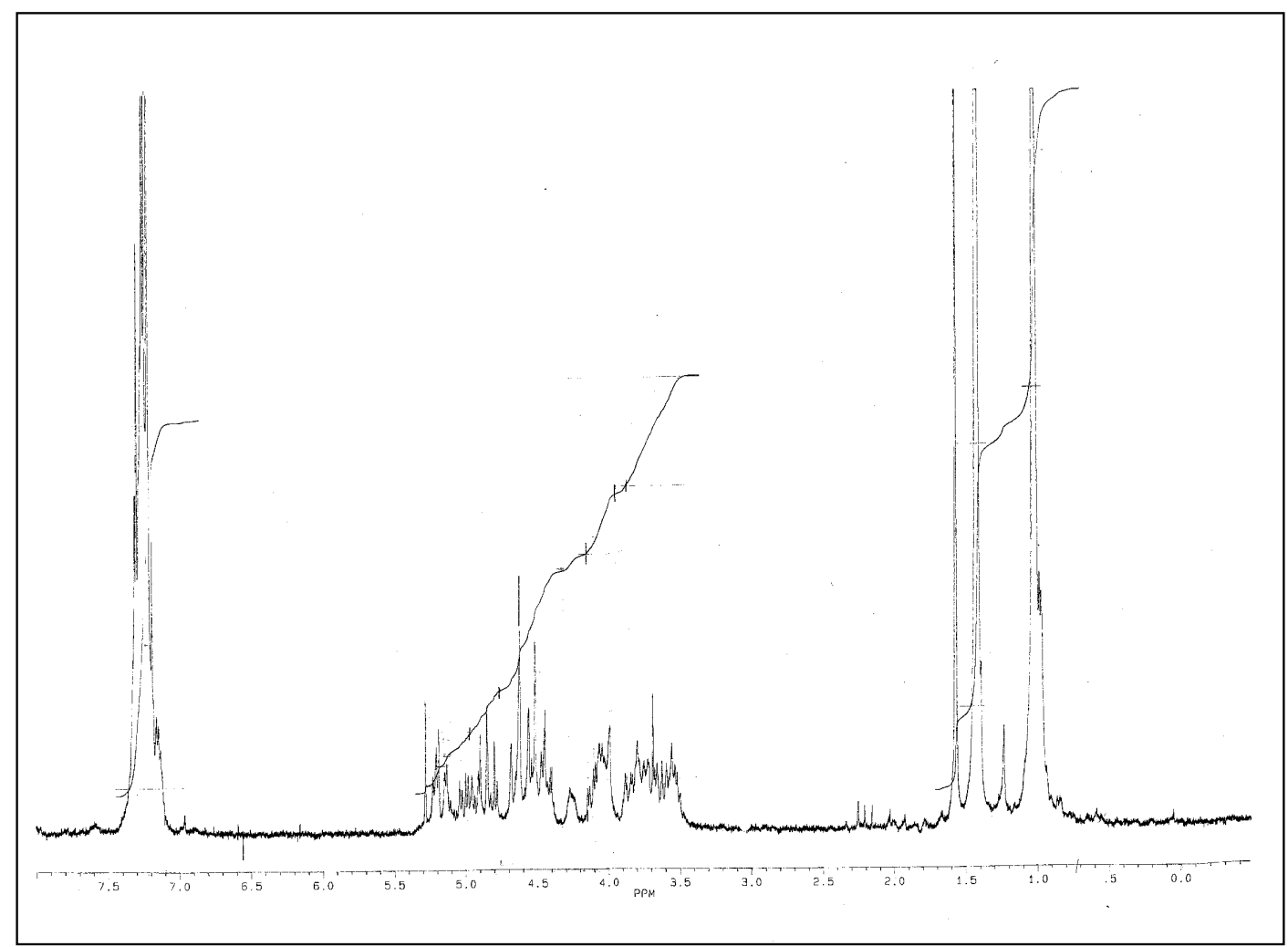

${ }^{13} \mathrm{C}$ NMR of compound 23a $\left(\mathrm{CDCl}_{3}, 63 \mathrm{MHz}\right)$ 


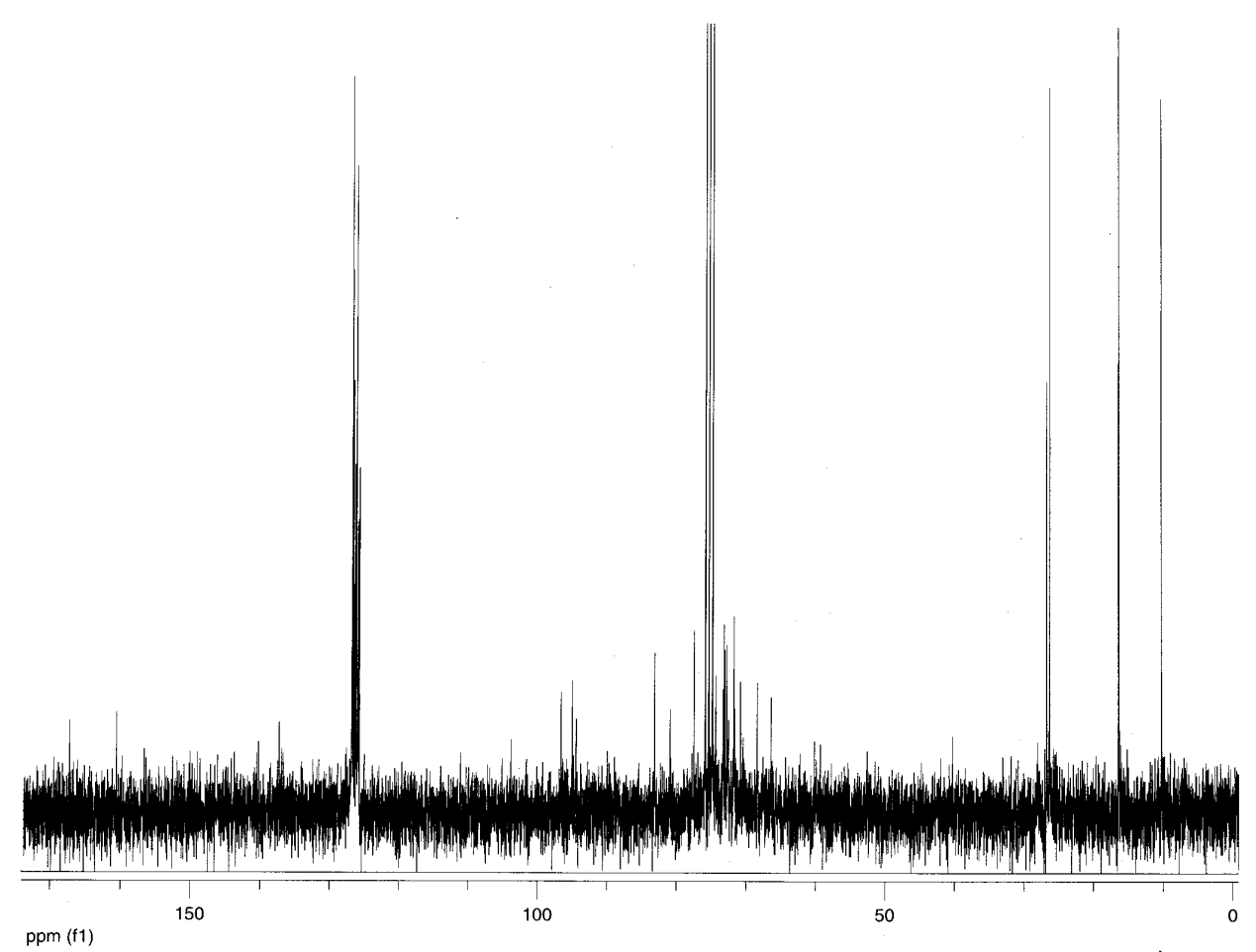

${ }^{1} \mathrm{H}$ NMR of compound $\mathbf{2 3 b}\left(\mathrm{CDCl}_{3}, 600 \mathrm{MHz}\right)$

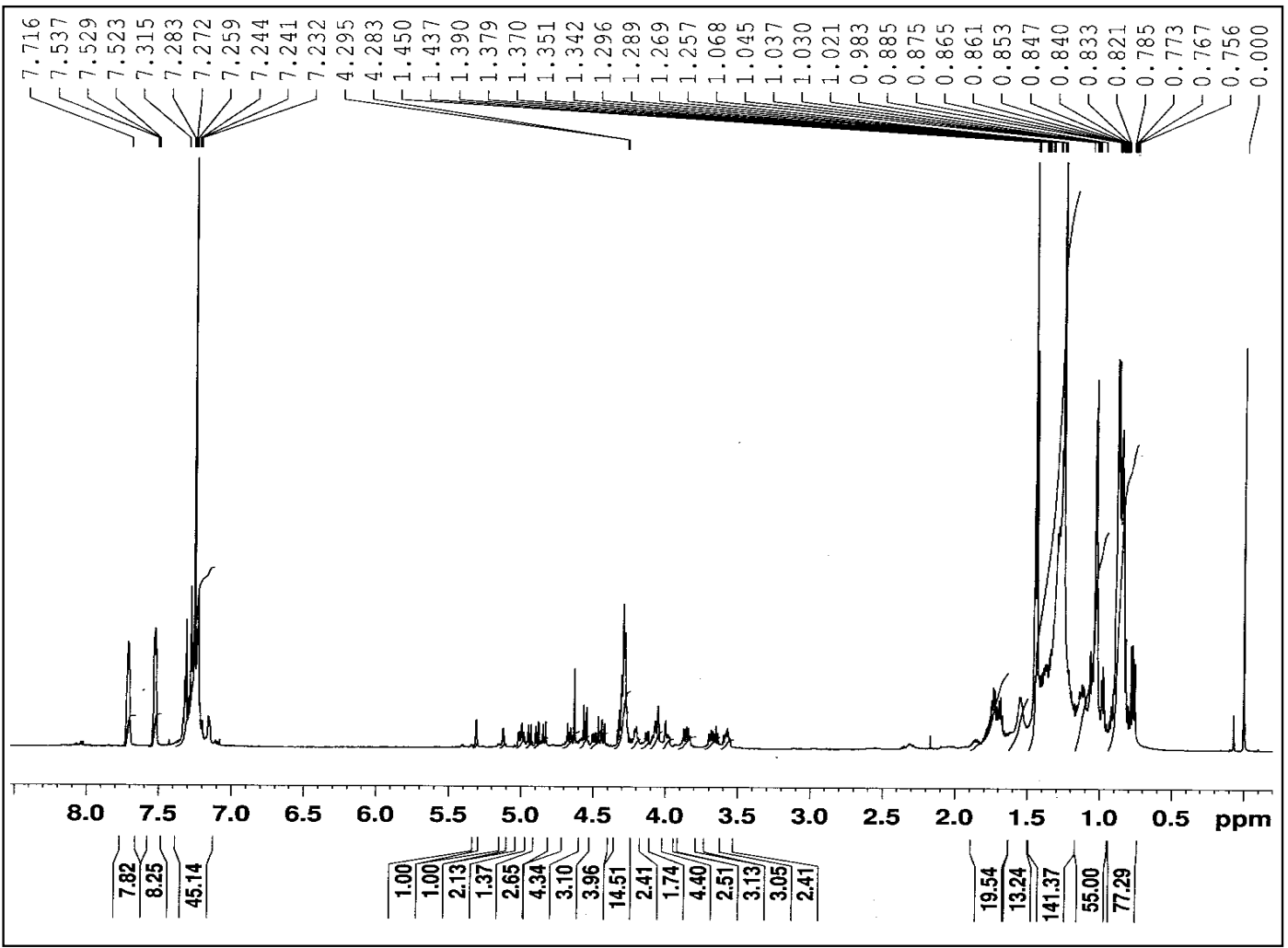


${ }^{13} \mathrm{C}$ NMR of compound 23b $\left(\mathrm{CDCl}_{3}, 151 \mathrm{MHz}\right)$

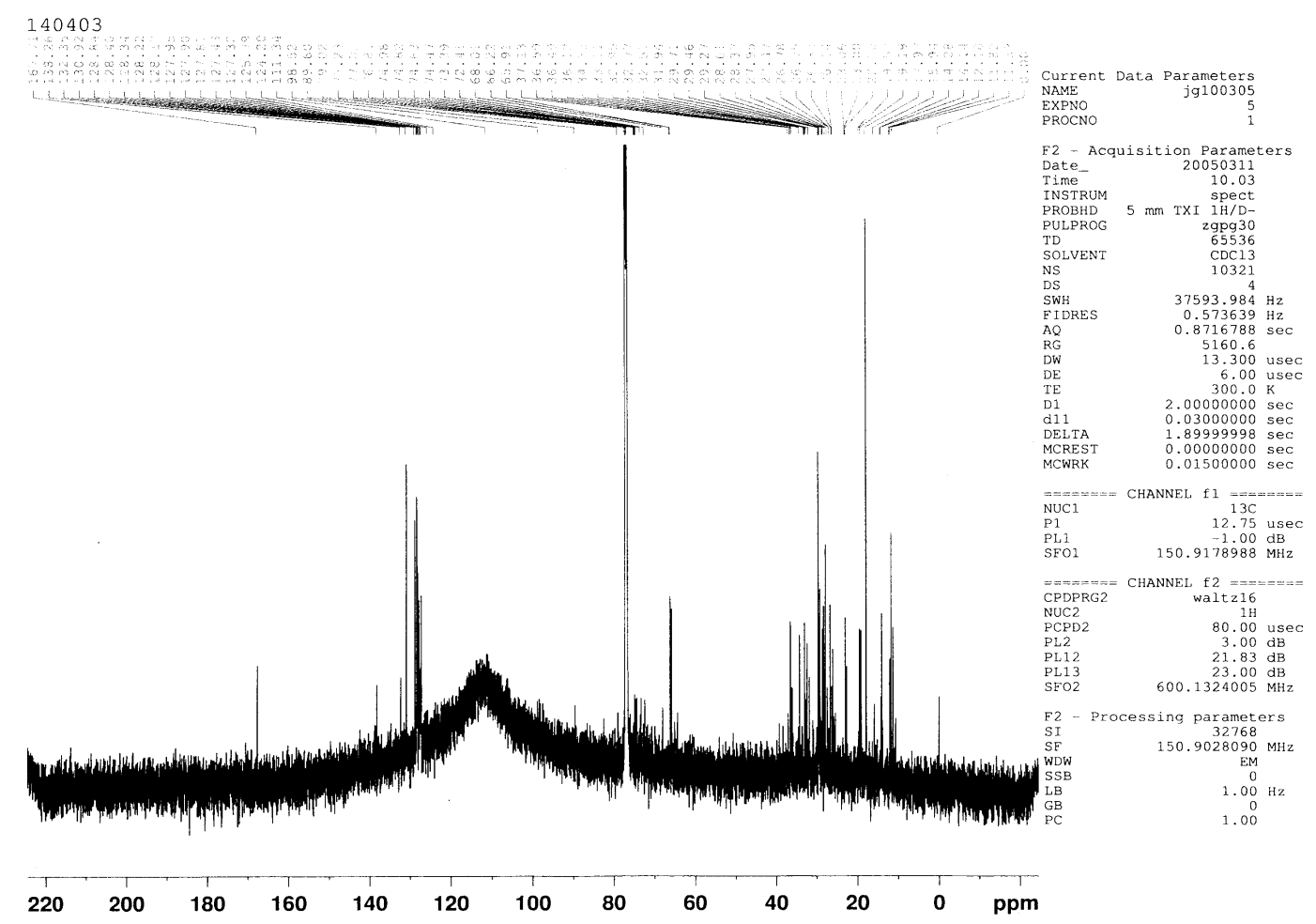

${ }^{1} \mathrm{H}$ NMR of compound $\mathbf{2 4 a}\left(\mathrm{CDCl}_{3}, 250 \mathrm{MHz}\right)$

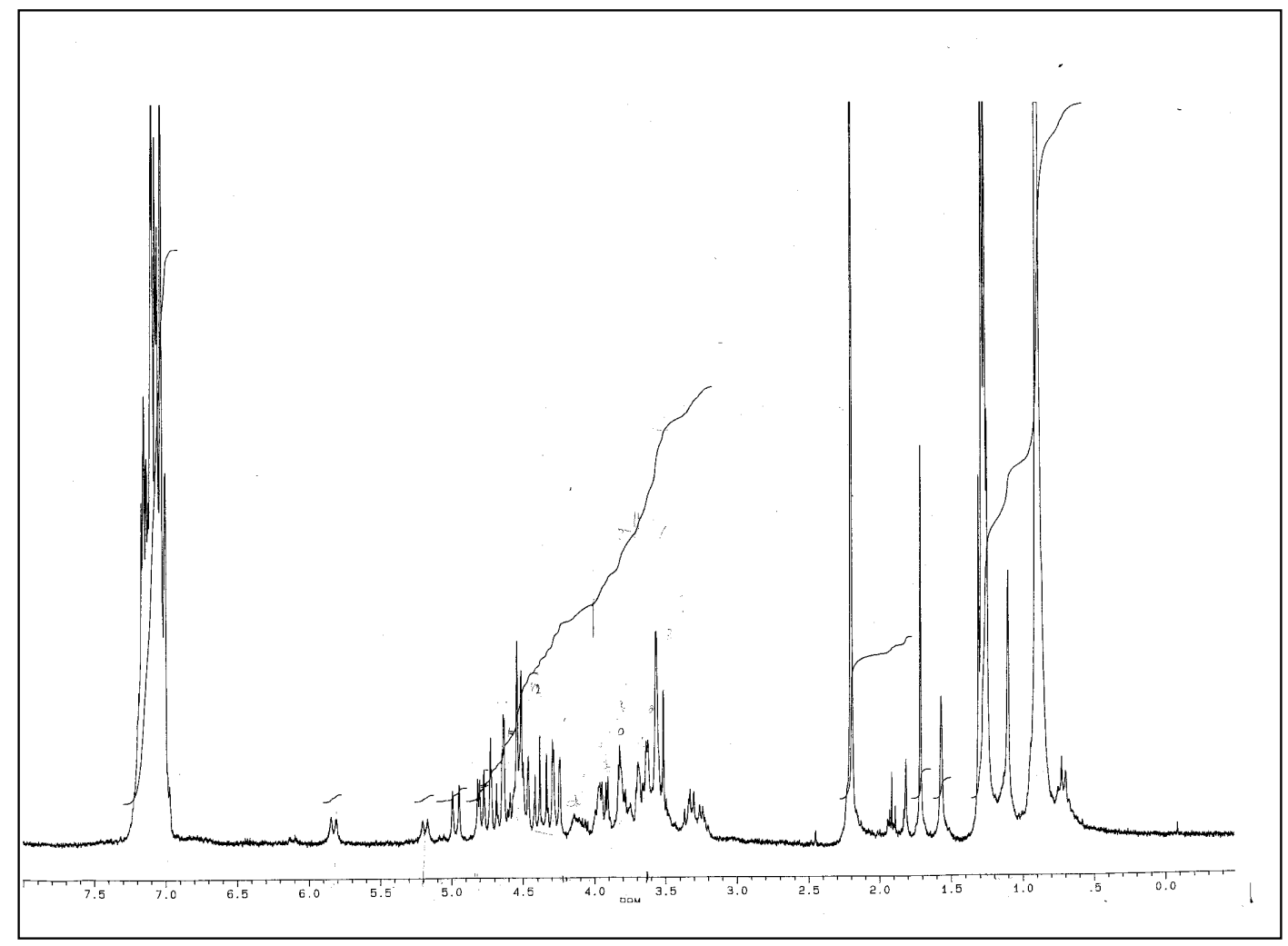


${ }^{13} \mathrm{C}$ NMR of compound 24a $\left(\mathrm{CDCl}_{3}, 63 \mathrm{MHz}\right)$

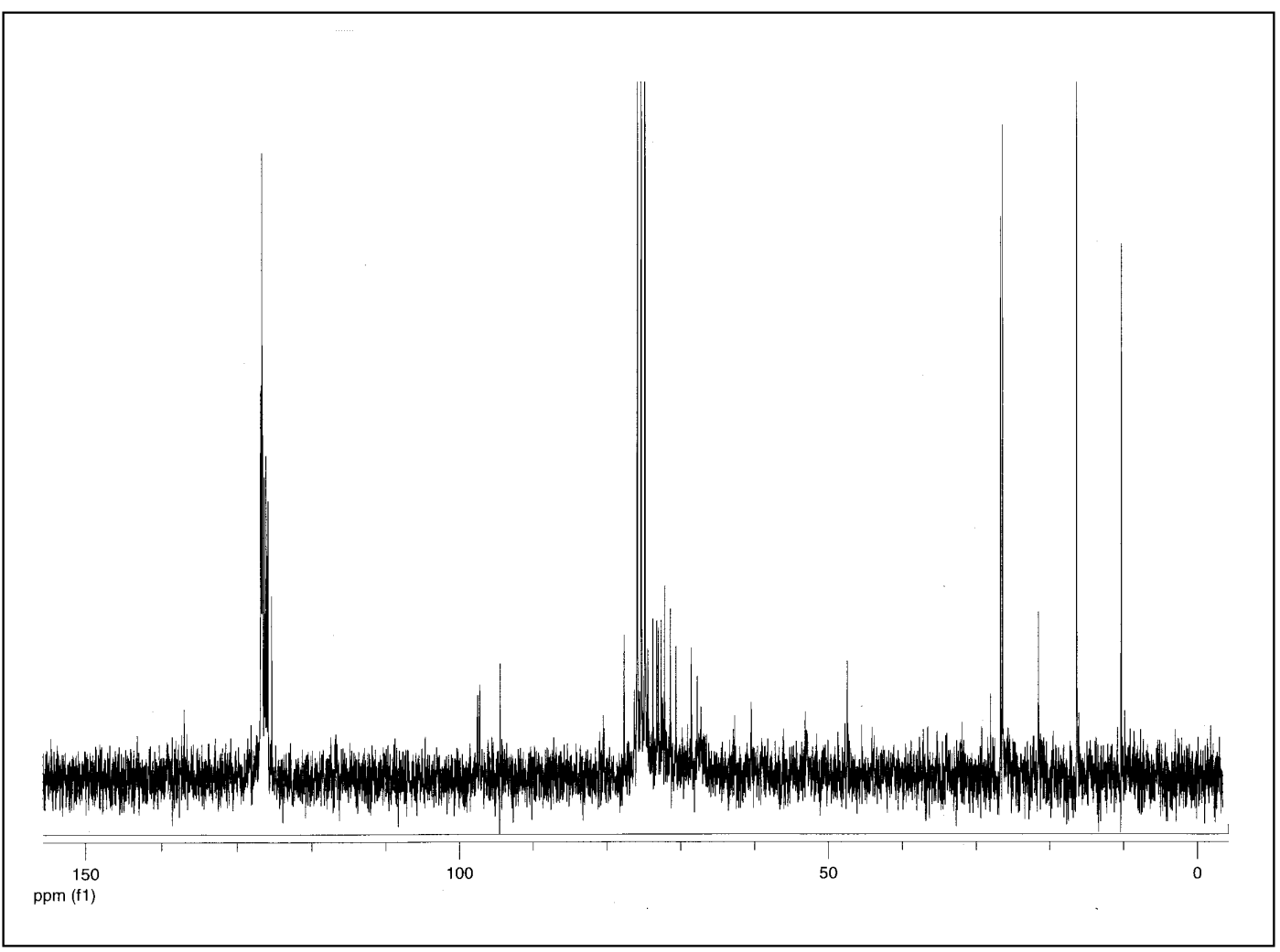

${ }^{1} \mathrm{H}$ NMR of compound $\mathbf{2 4 b}\left(\mathrm{CDCl}_{3}, 250 \mathrm{MHz}\right)$

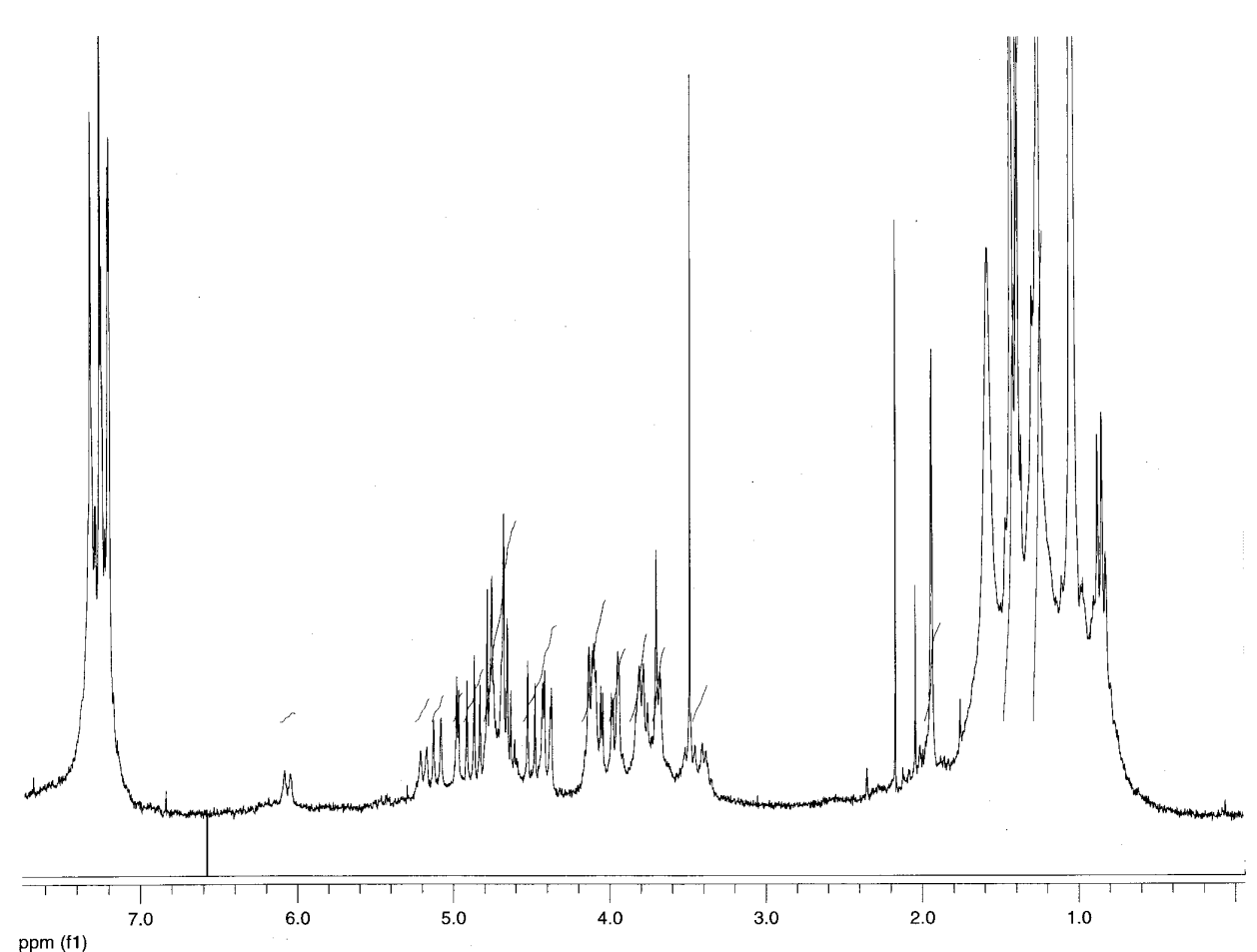


${ }^{13} \mathrm{C}$ NMR of compound $24 \mathbf{b}\left(\mathrm{CDCl}_{3}, 151 \mathrm{MHz}\right)$
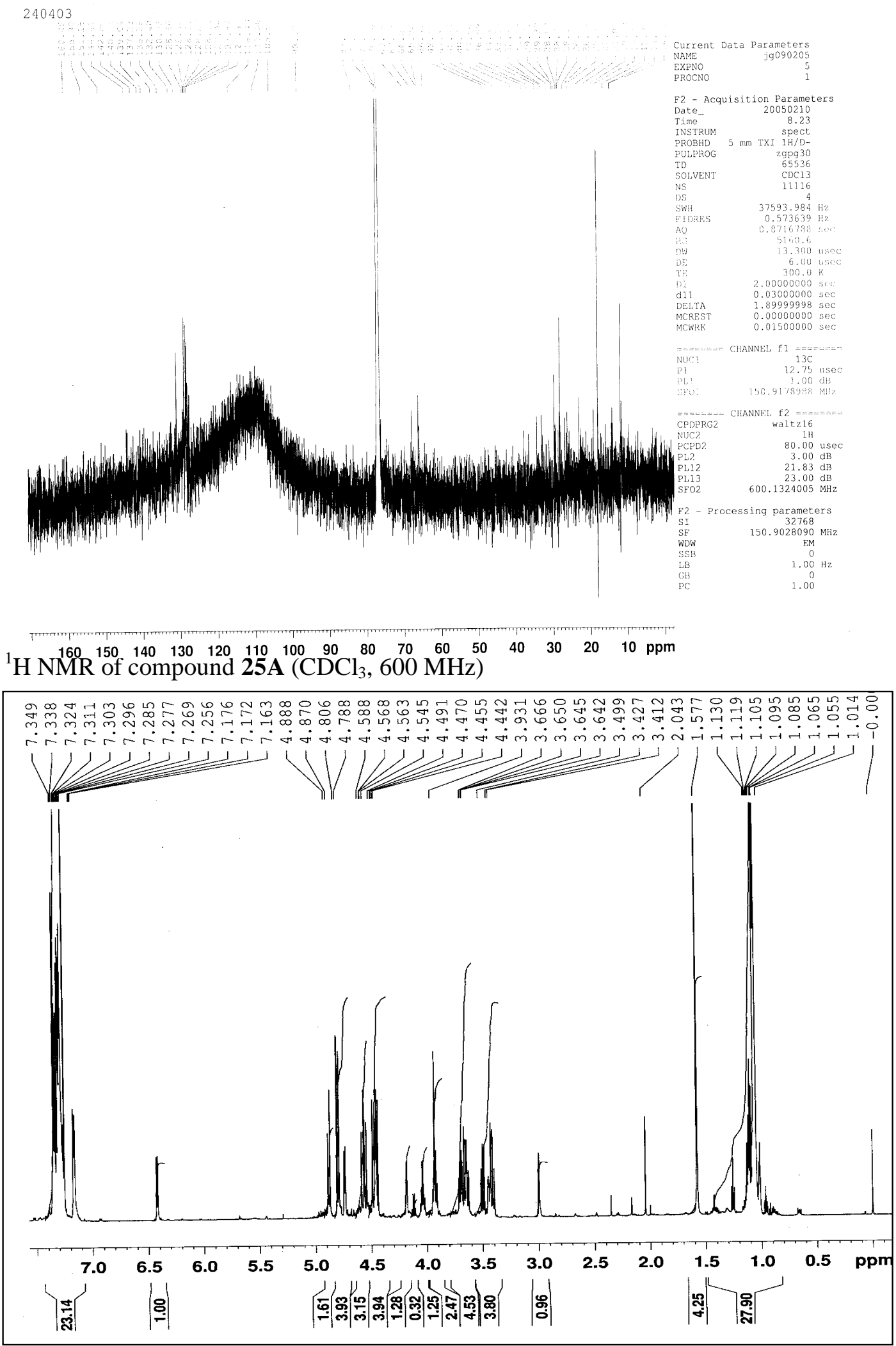

${ }^{13} \mathrm{C}$ NMR of compound $25 \mathrm{~A}\left(\mathrm{CDCl}_{3}, 151 \mathrm{MHz}\right)$ 


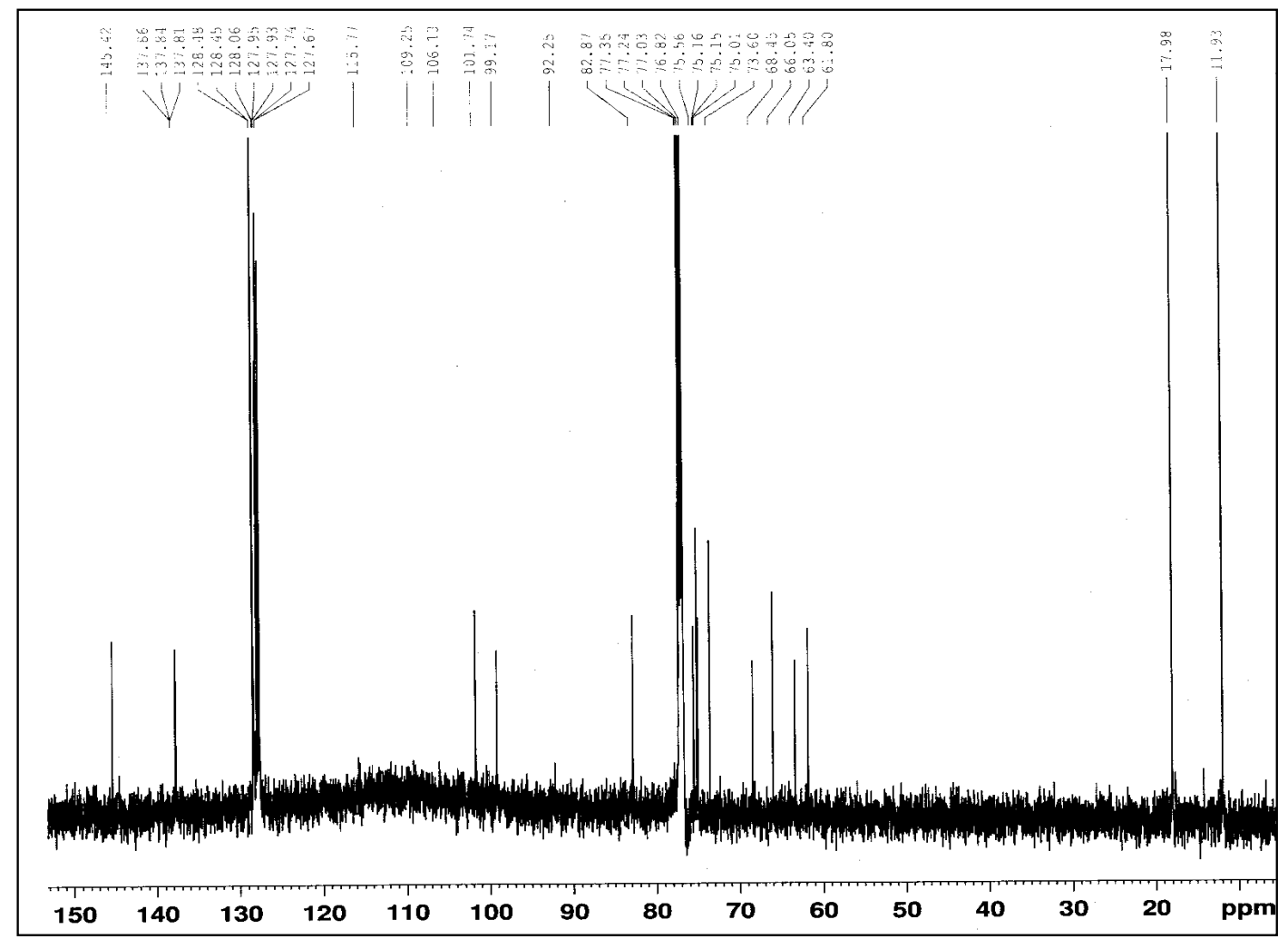

${ }^{1} \mathrm{H}$ NMR of compound 25B $\left(\mathrm{CDCl}_{3}, 250 \mathrm{MHz}\right)$

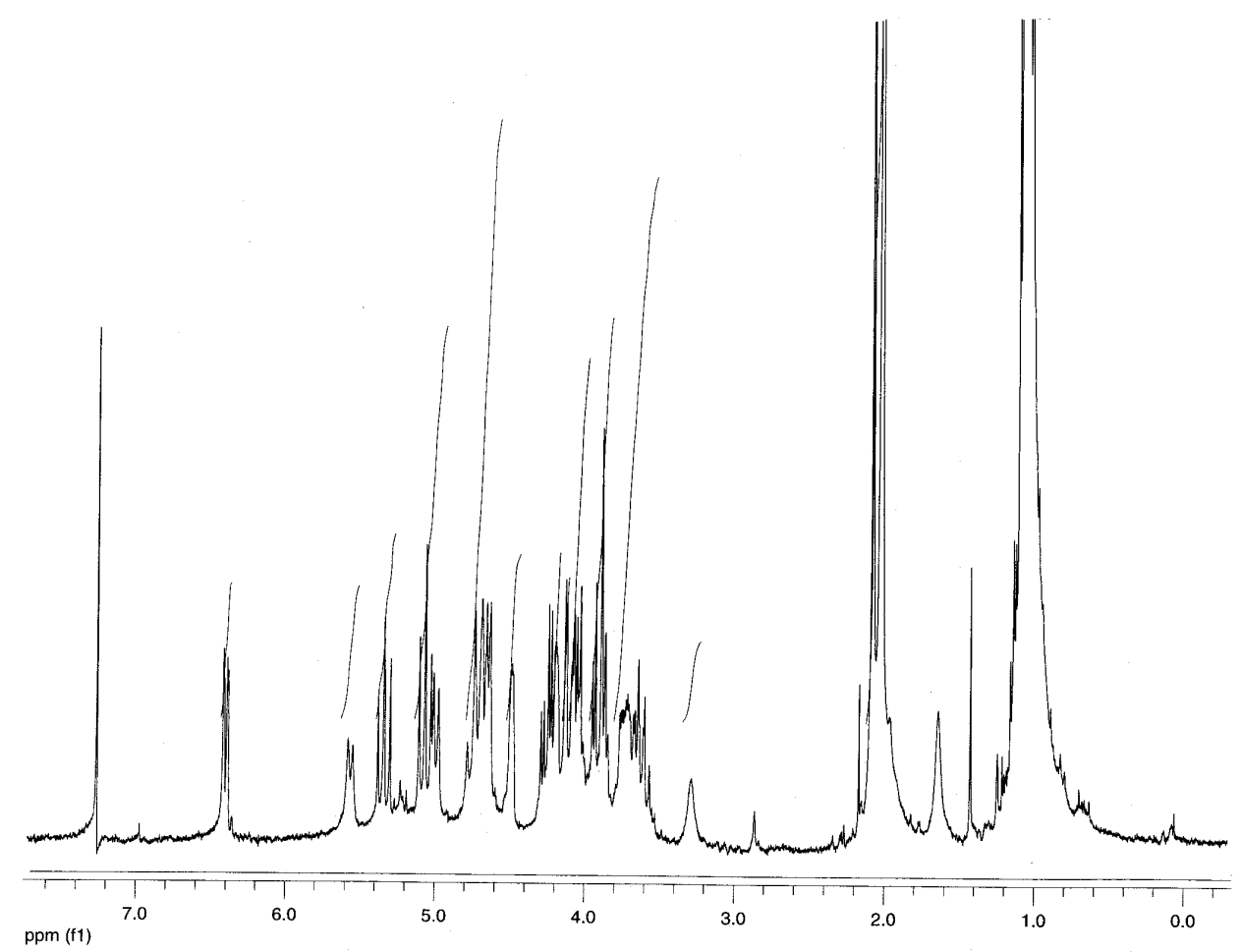


${ }^{1} \mathrm{H}$ NMR of compound $25 \mathrm{C}\left(\mathrm{CDCl}_{3}, 250 \mathrm{MHz}\right)$

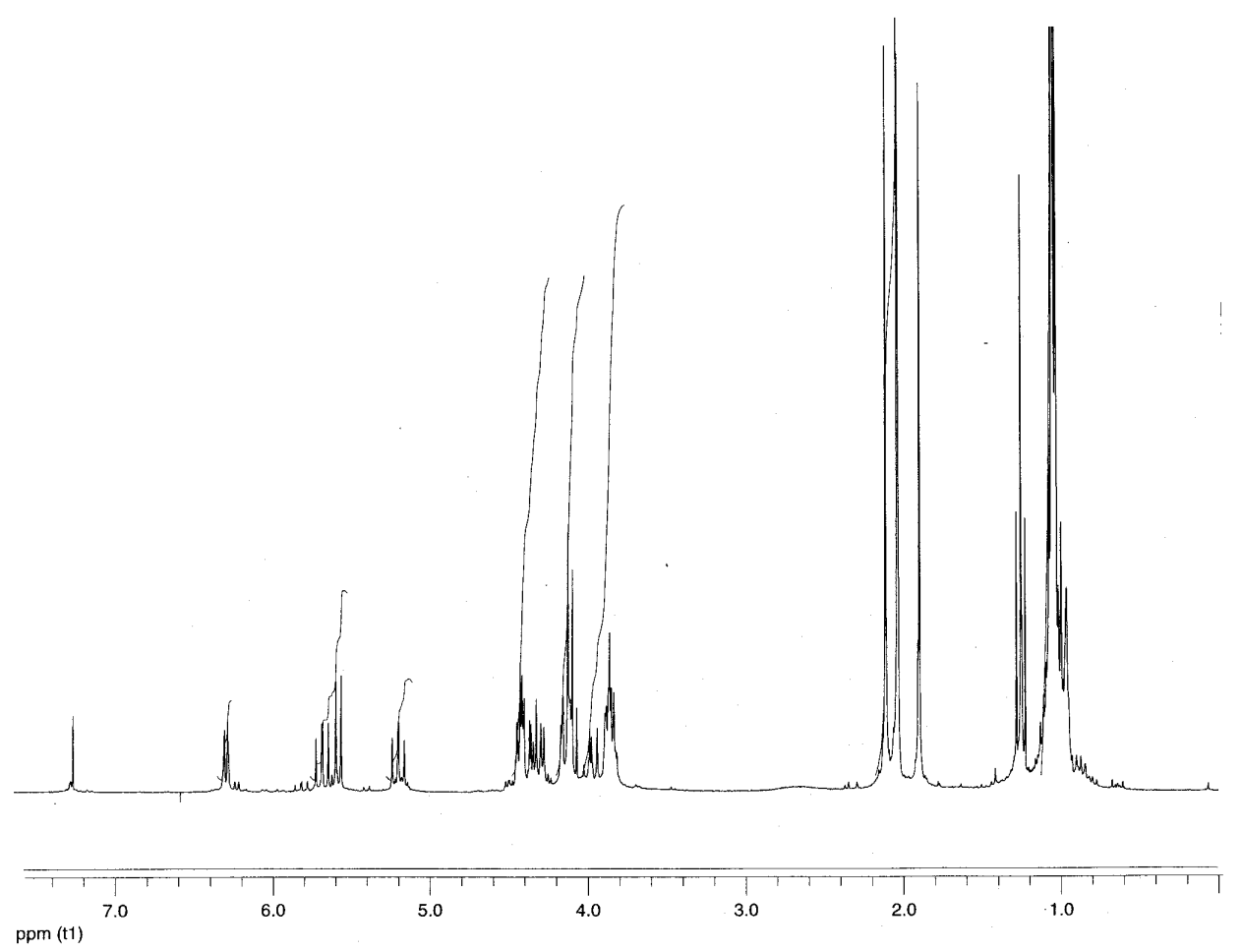

${ }^{1} \mathrm{H}$ NMR of compound $26\left(\mathrm{CDCl}_{3}, 600 \mathrm{MHz}\right)$

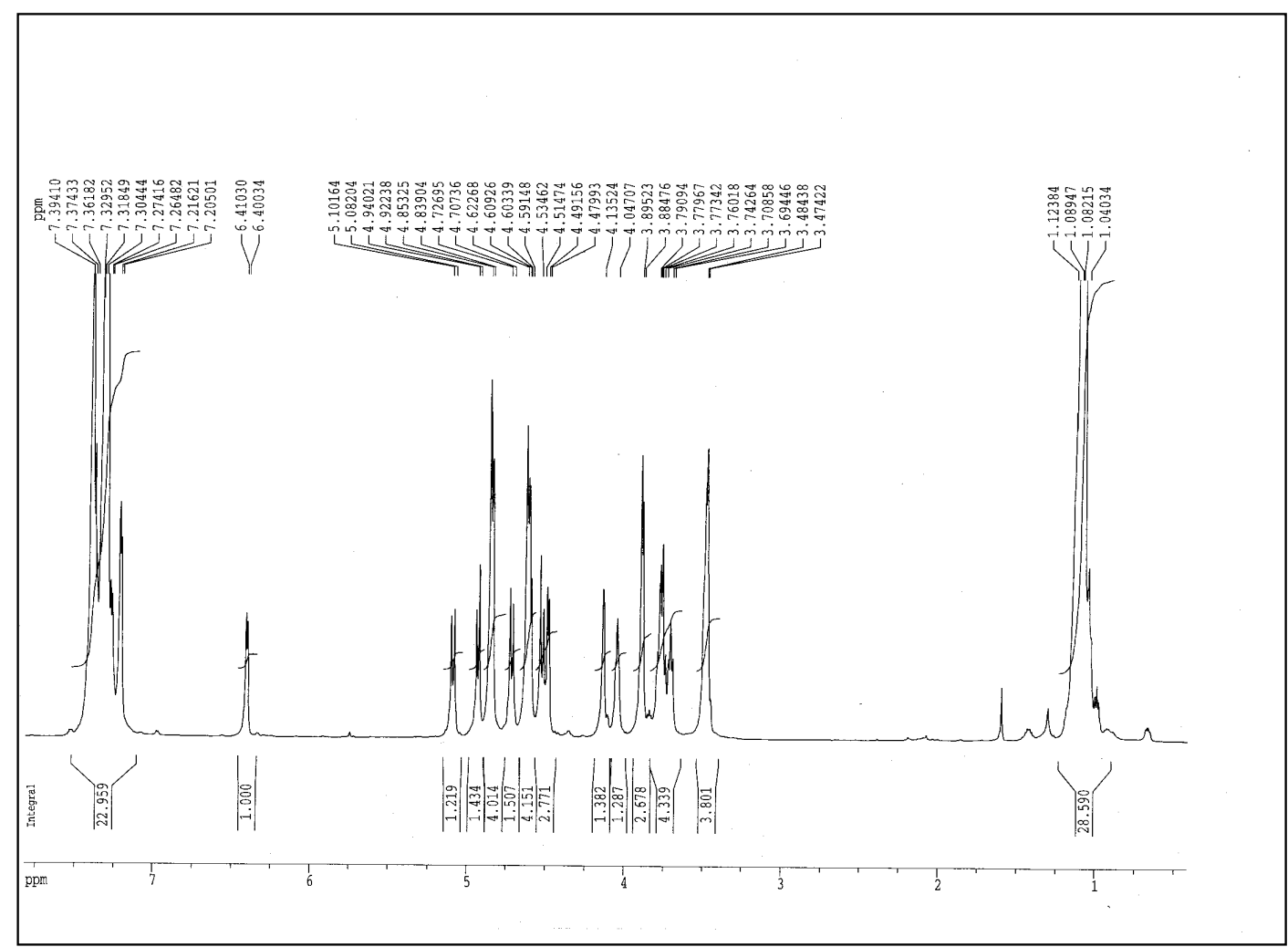


${ }^{13} \mathrm{C}$ NMR of compound $26\left(\mathrm{CDCl}_{3}, 151 \mathrm{MHz}\right)$

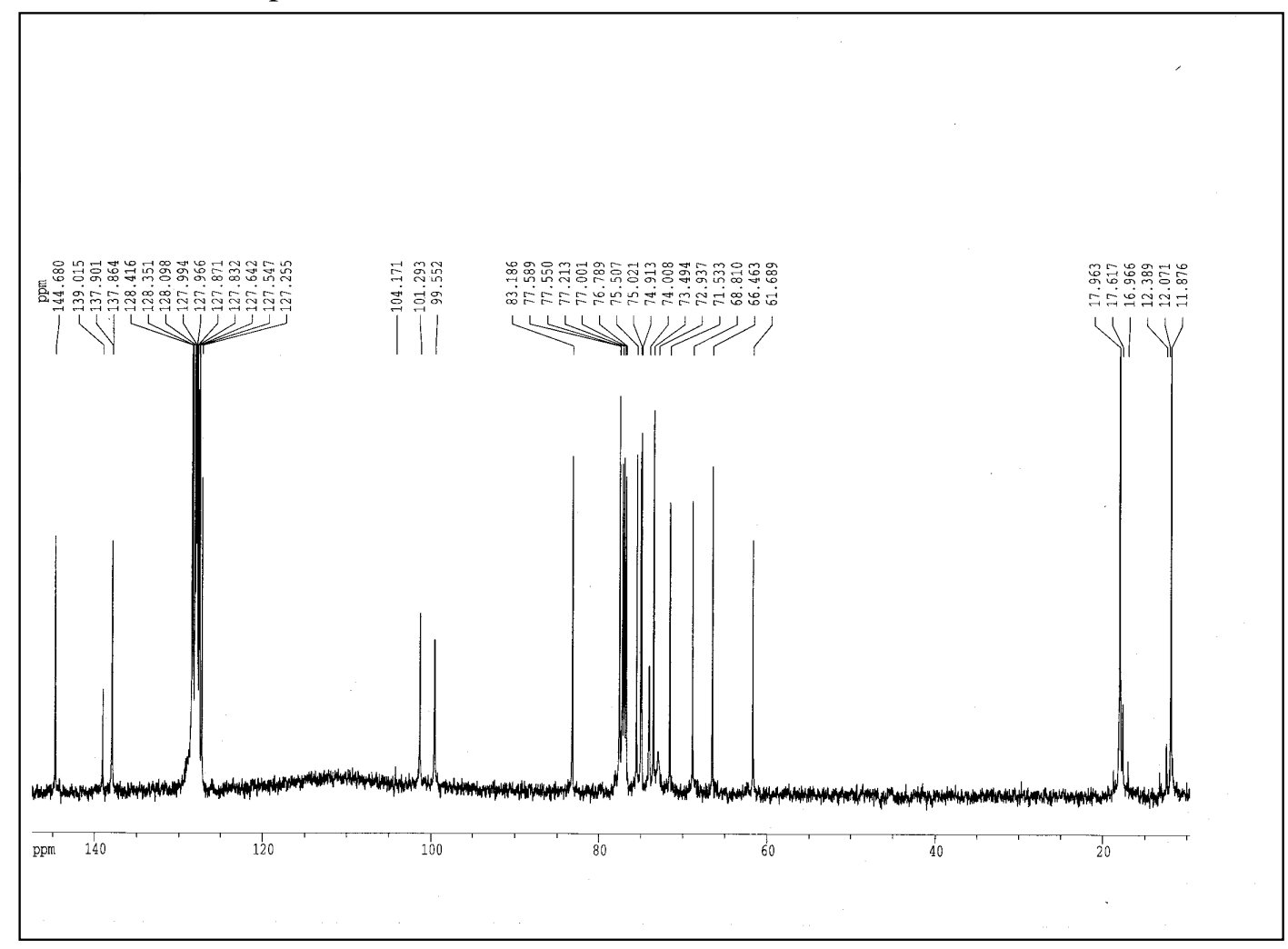

${ }^{1} \mathrm{H}$ NMR of compound $27\left(\mathrm{CDCl}_{3}, 250 \mathrm{MHz}\right)$

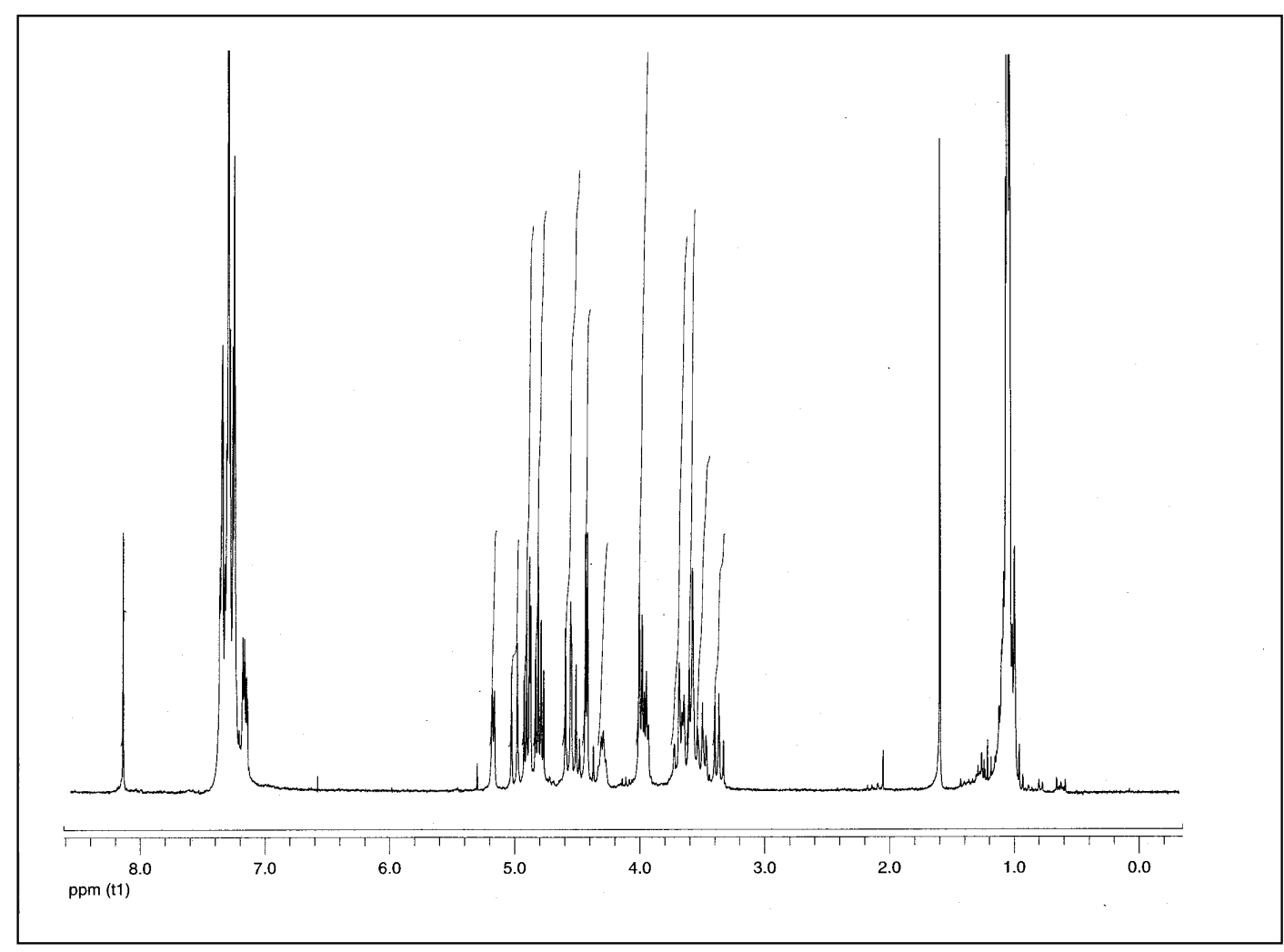


${ }^{13} \mathrm{C}$ NMR of compound $27\left(\mathrm{CDCl}_{3}, 63 \mathrm{MHz}\right)$

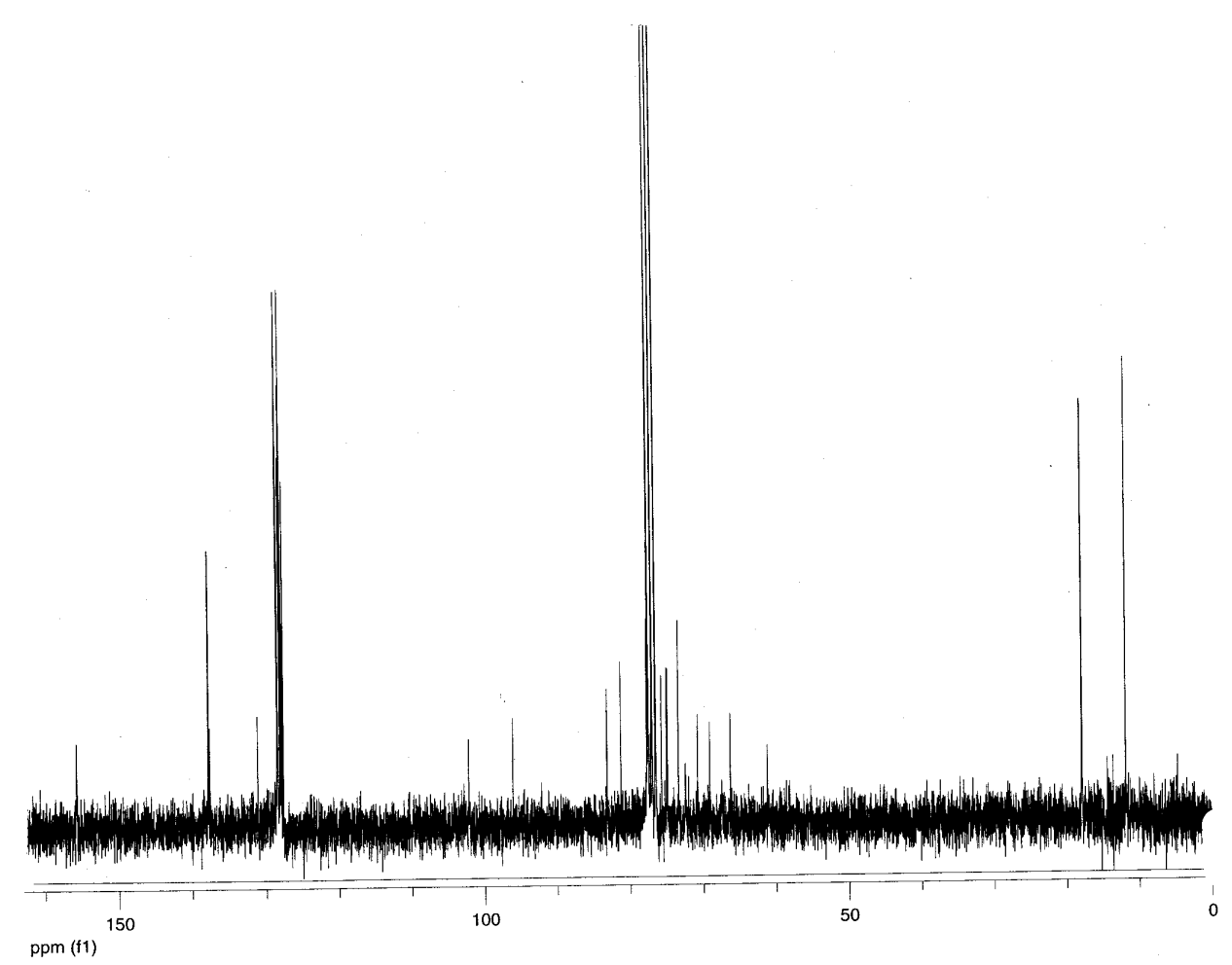

${ }^{1} \mathrm{H}$ NMR of compound 28a $\left(\mathrm{CDCl}_{3}, 600 \mathrm{MHz}\right)$

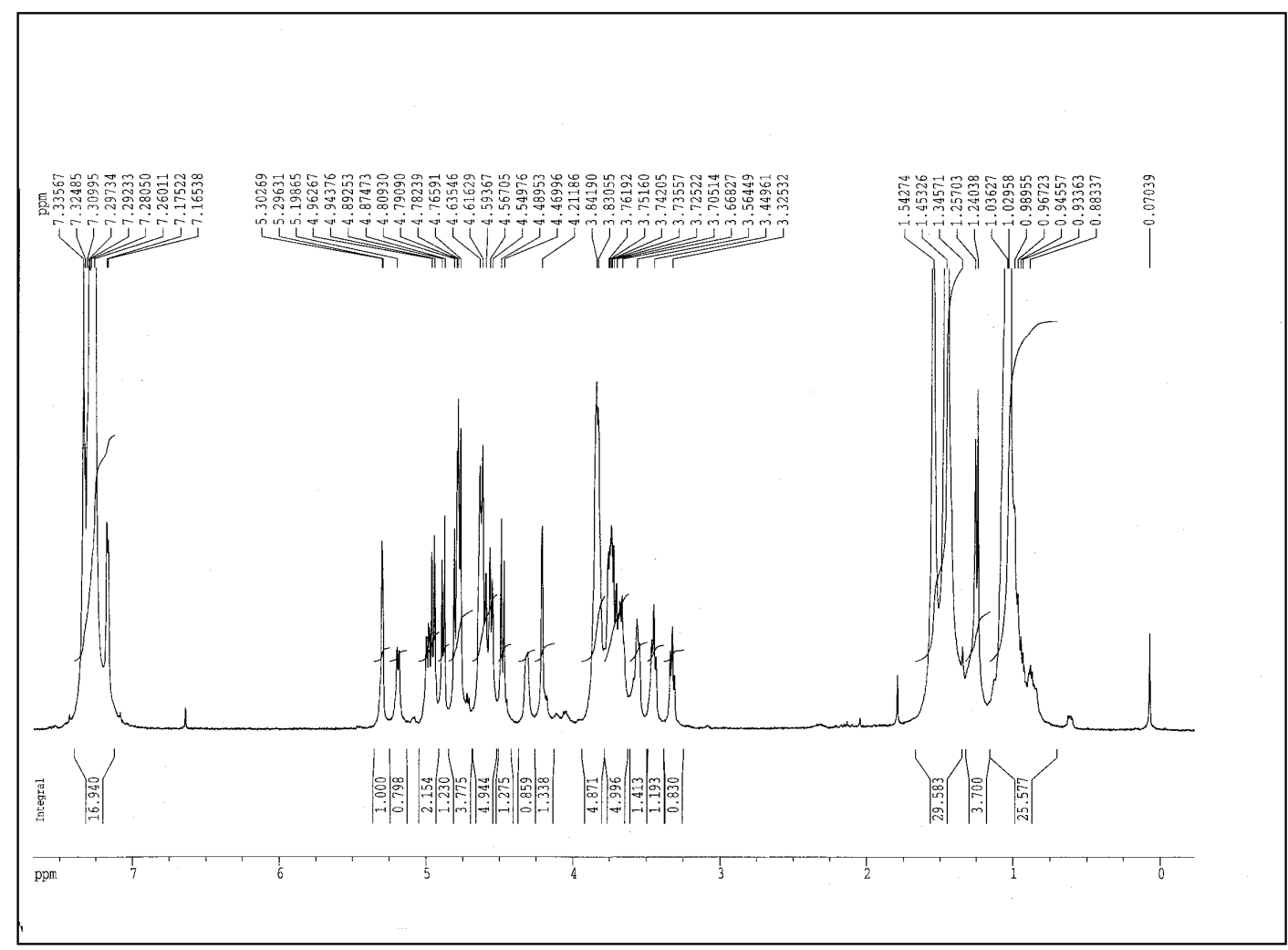




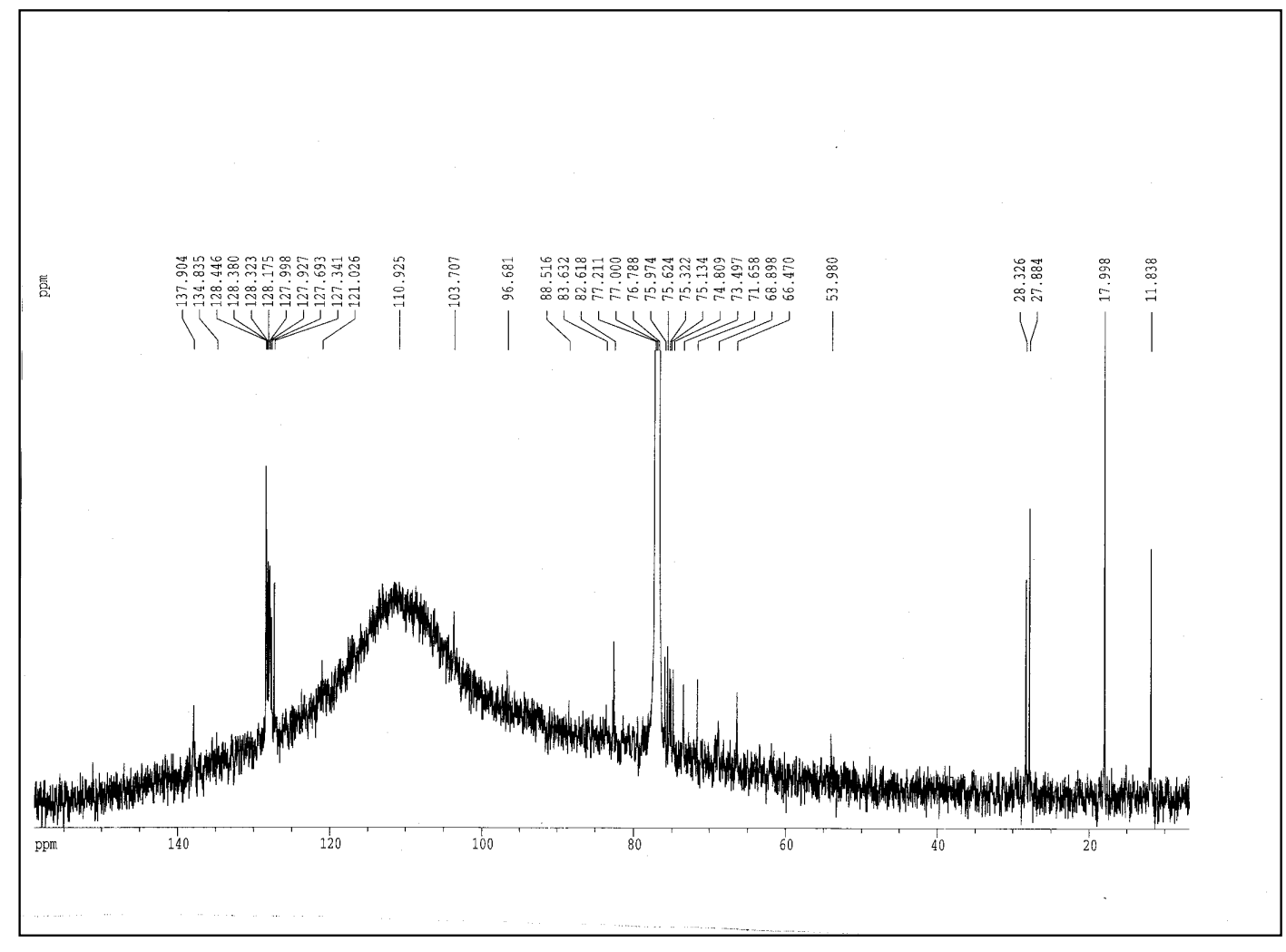

${ }^{1} \mathrm{H}$ NMR of compound $\mathbf{3 0}\left(\mathrm{CDCl}_{3}, 250 \mathrm{MHz}\right)$

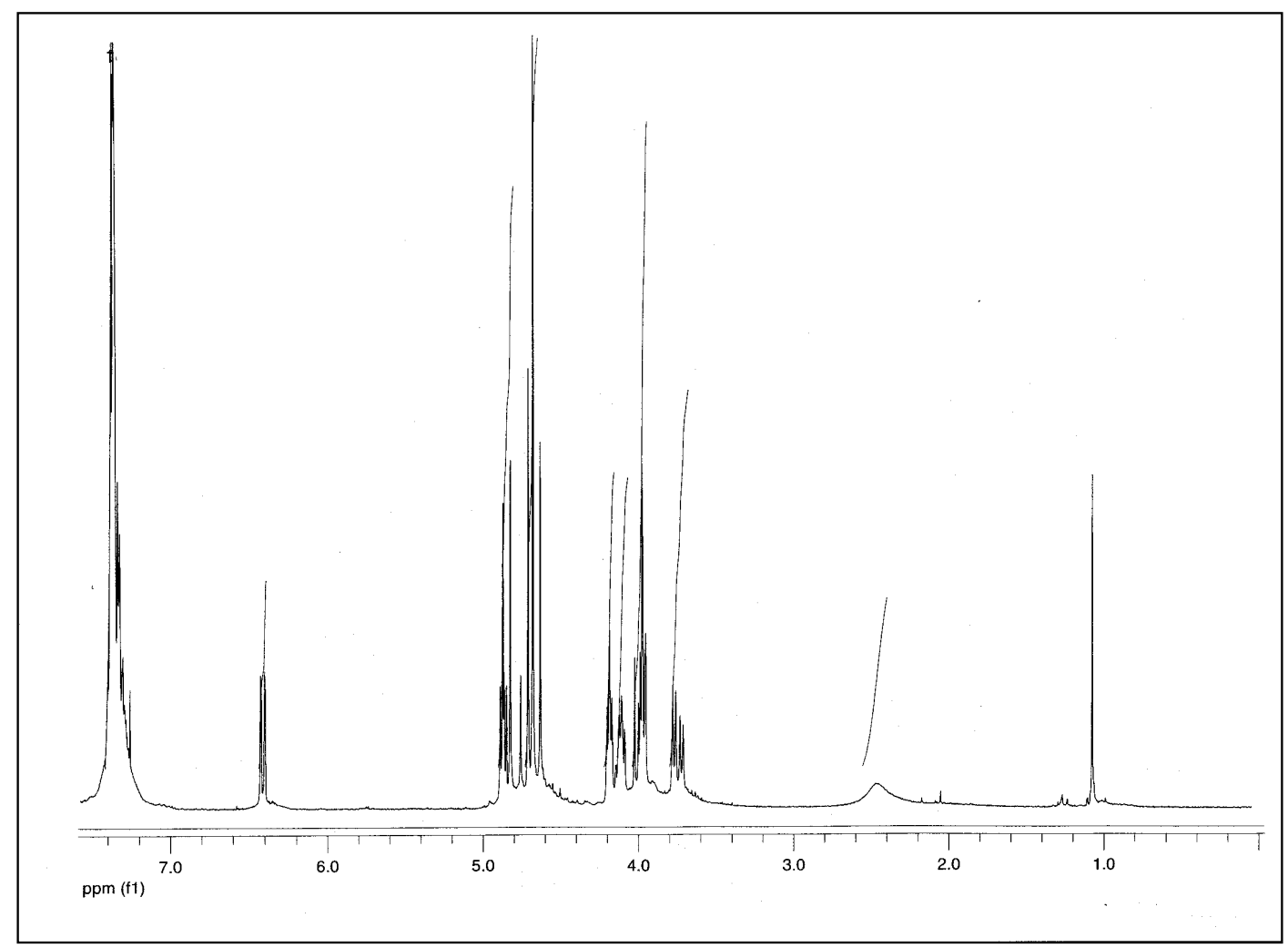


${ }^{1} \mathrm{H}$ NMR of compound $\mathbf{3 1}\left(\mathrm{CDCl}_{3}, 250 \mathrm{MHz}\right)$

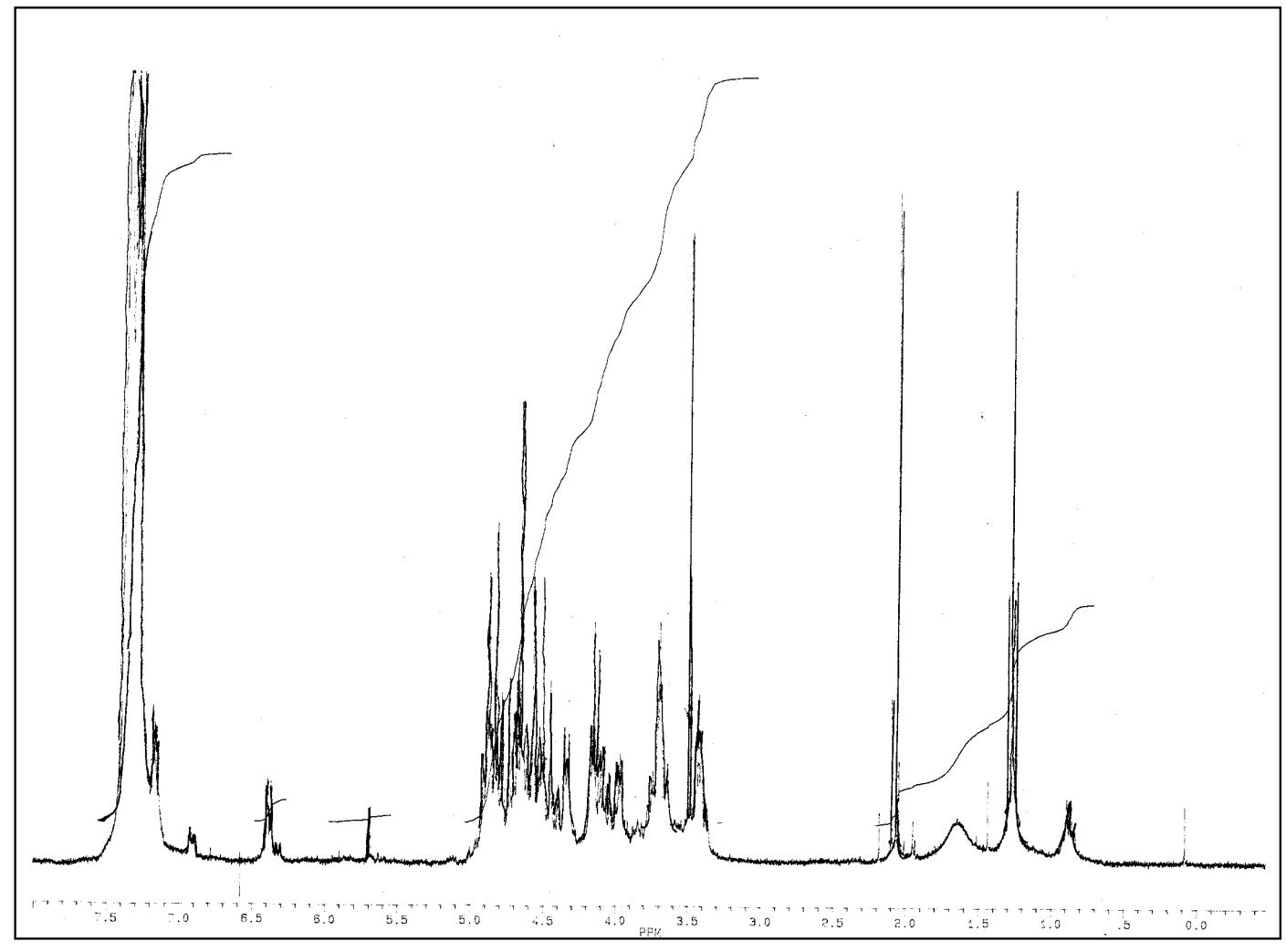

${ }^{1} \mathrm{H}$ NMR of compound $32\left(\mathrm{CDCl}_{3}, 600 \mathrm{MHz}\right)$

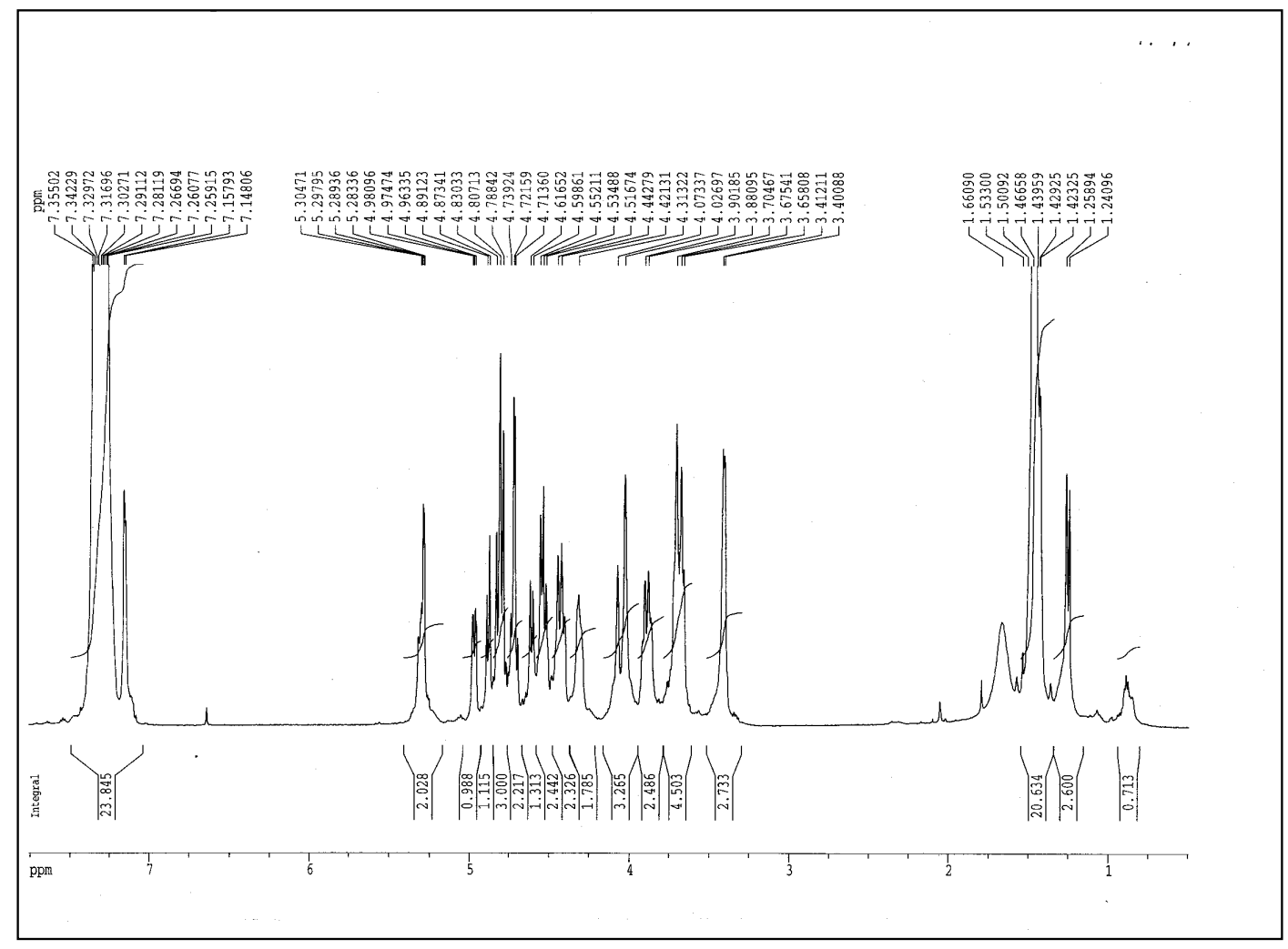

${ }^{1} \mathrm{H}$ NMR of compound 33a $\left(\mathrm{CDCl}_{3}, 600 \mathrm{MHz}\right)$ 


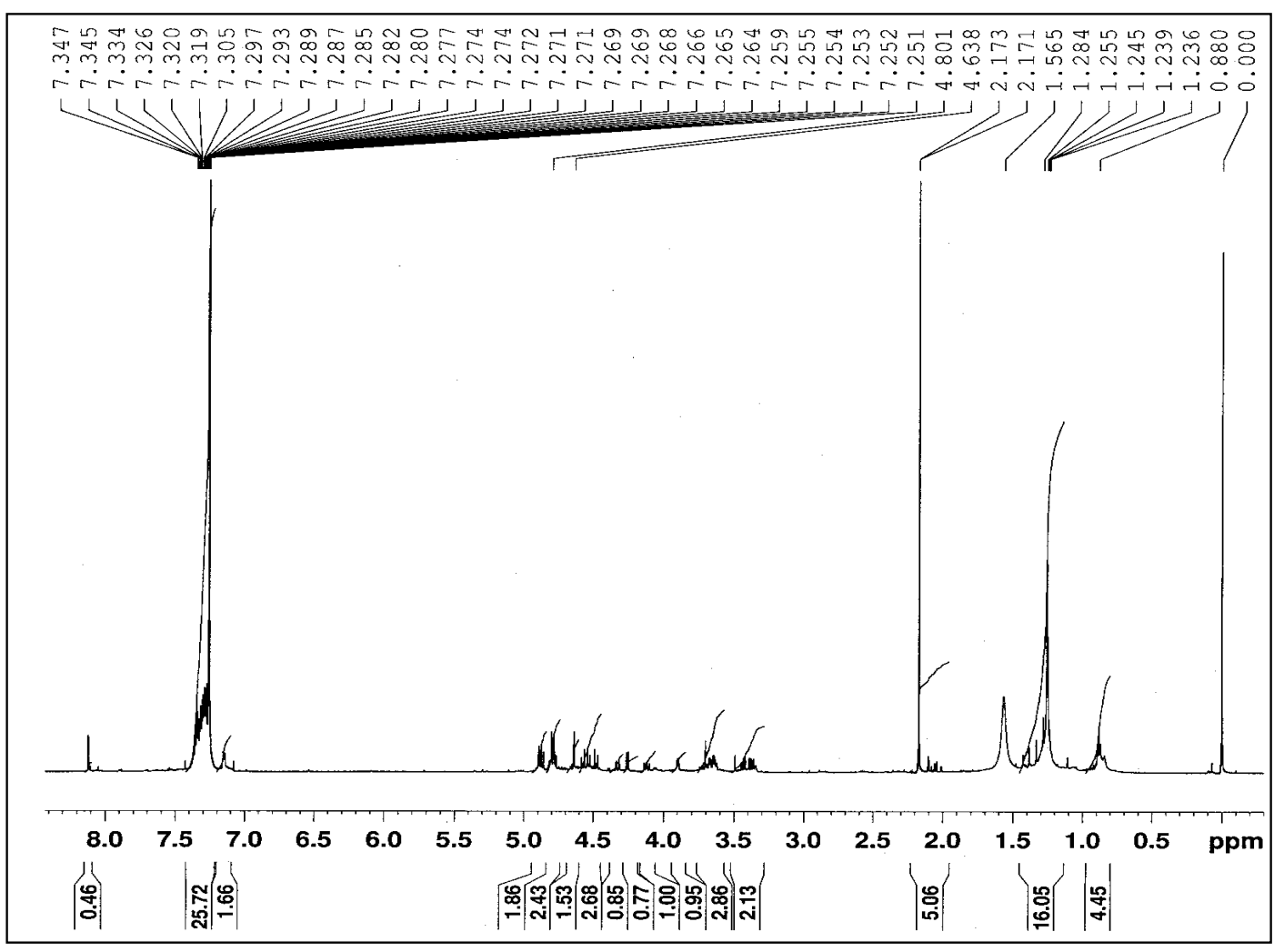

${ }^{13} \mathrm{C}$ NMR of compound 33a $\left(\mathrm{CDCl}_{3}, 151 \mathrm{MHz}\right)$

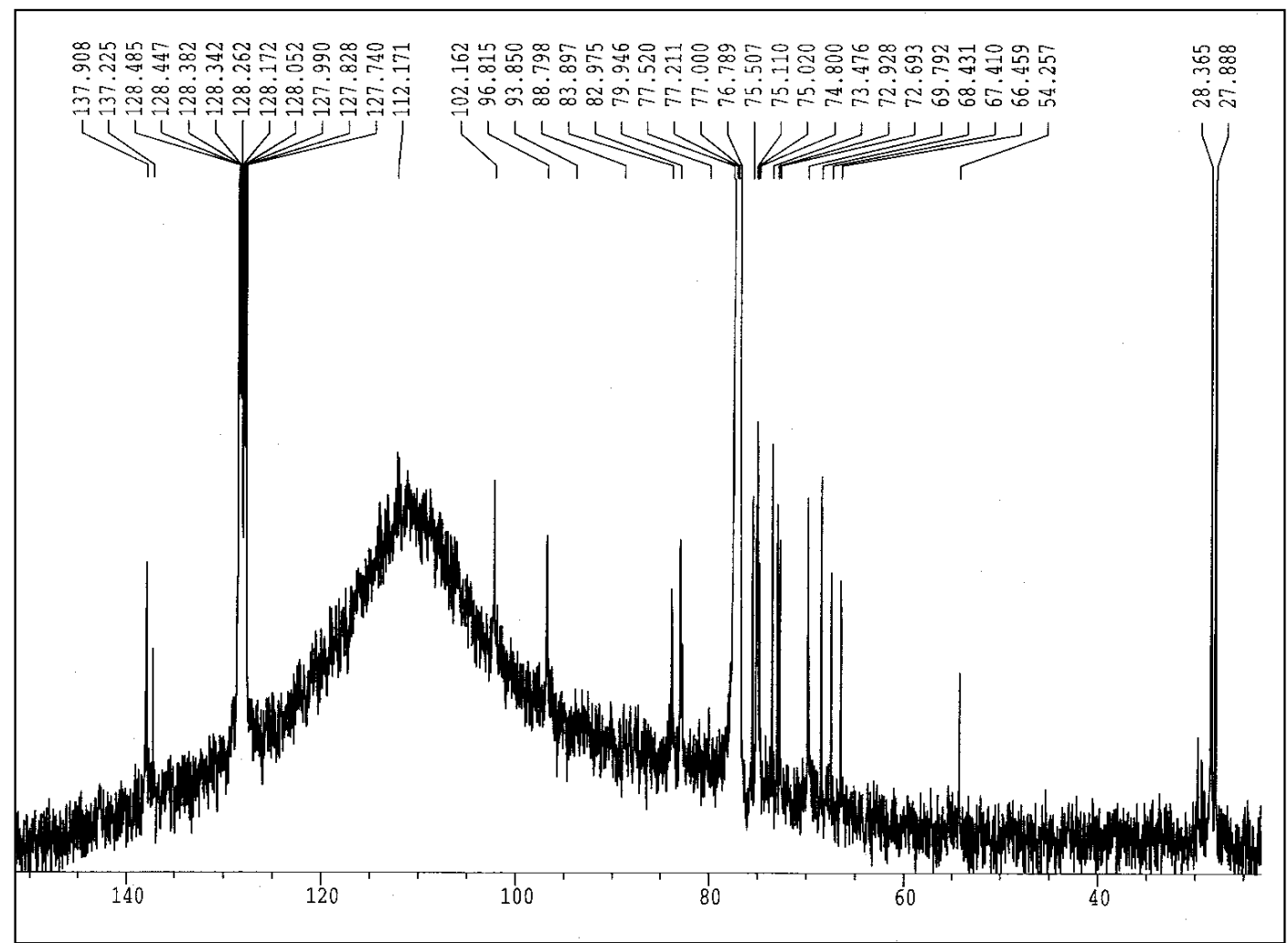

${ }^{1} \mathrm{H}$ NMR of compound $\mathbf{3 3 b}\left(\mathrm{CDCl}_{3}, 600 \mathrm{MHz}\right)$ 


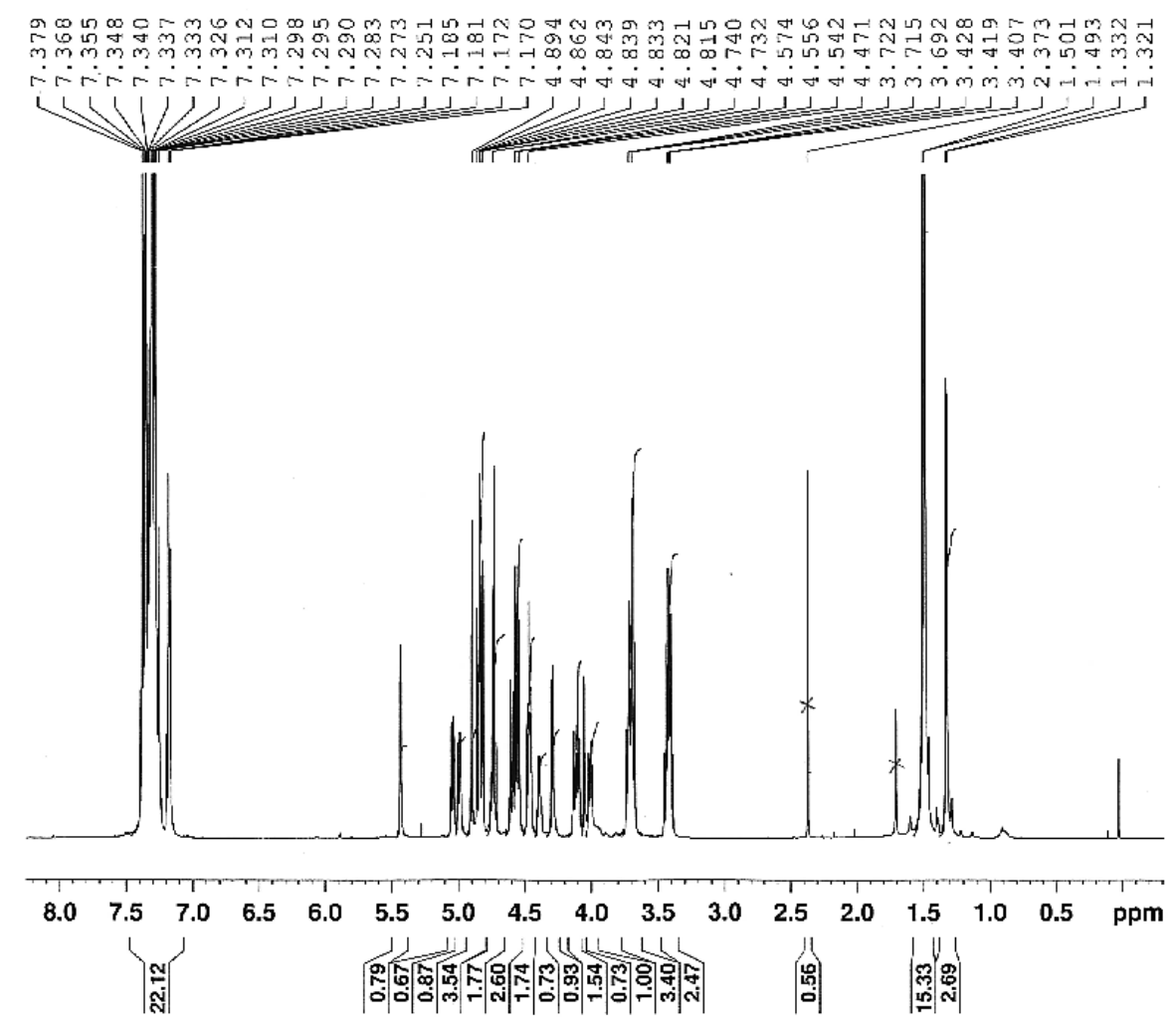

${ }^{13} \mathrm{C}$ NMR of compound 33b $\left(\mathrm{CDCl}_{3}, 151 \mathrm{MHz}\right)$

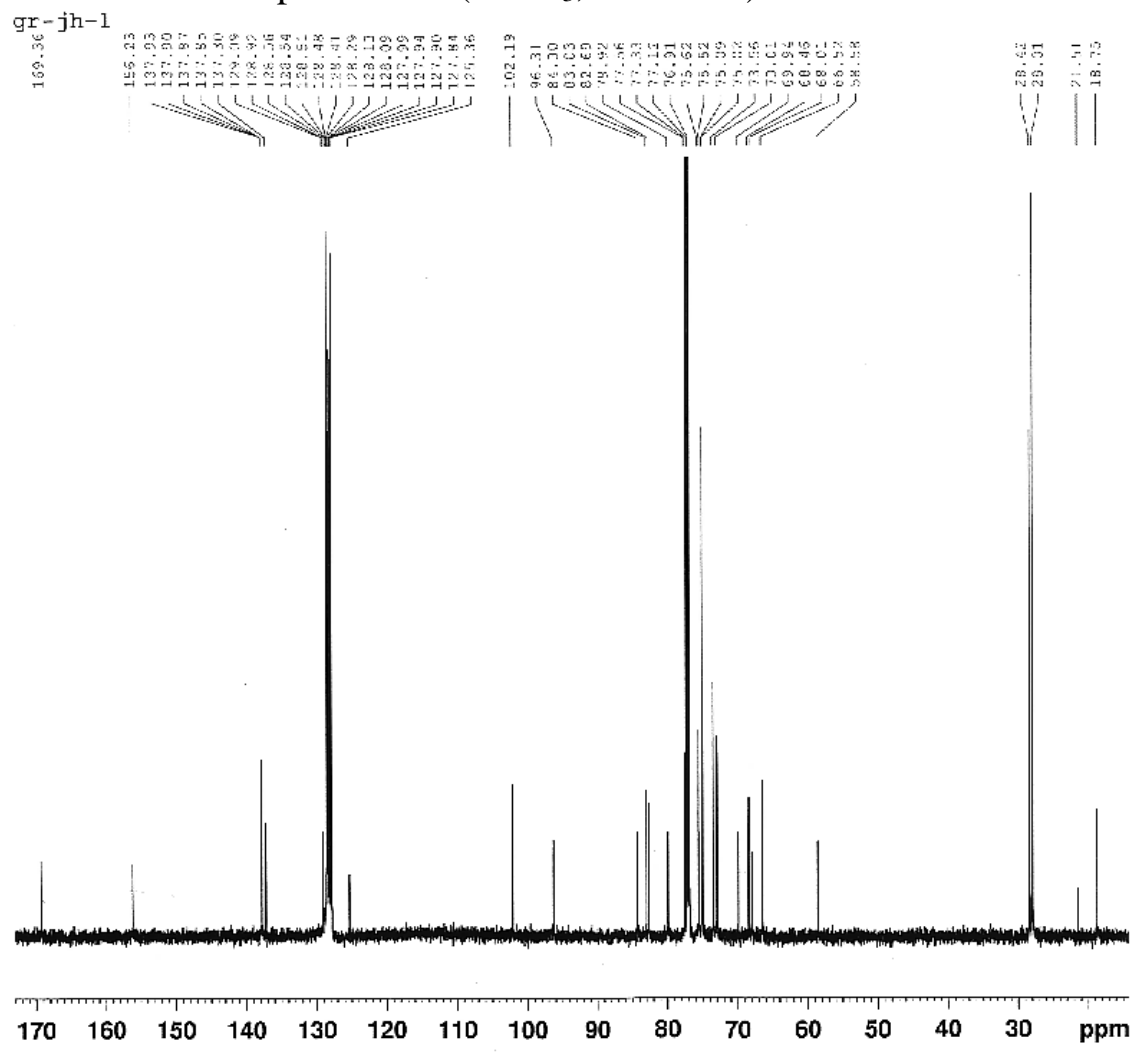

${ }^{1} \mathrm{H}$ NMR of compound 34a $\left(\mathrm{CDCl}_{3}, 600 \mathrm{MHz}\right)$ 


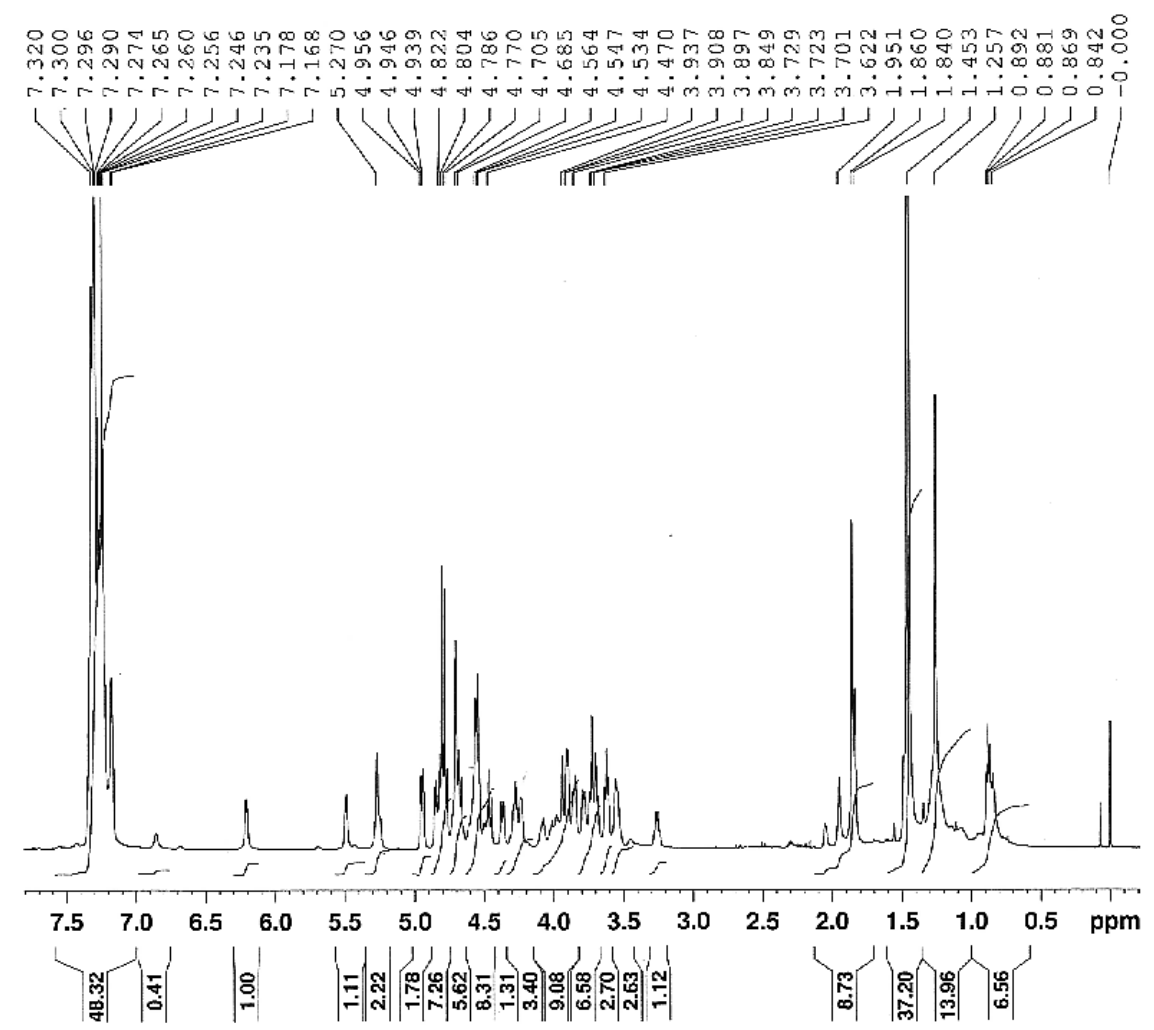

${ }^{13} \mathrm{C}$ NMR of compound 34a $\left(\mathrm{CDCl}_{3}, 151 \mathrm{MHz}\right)$

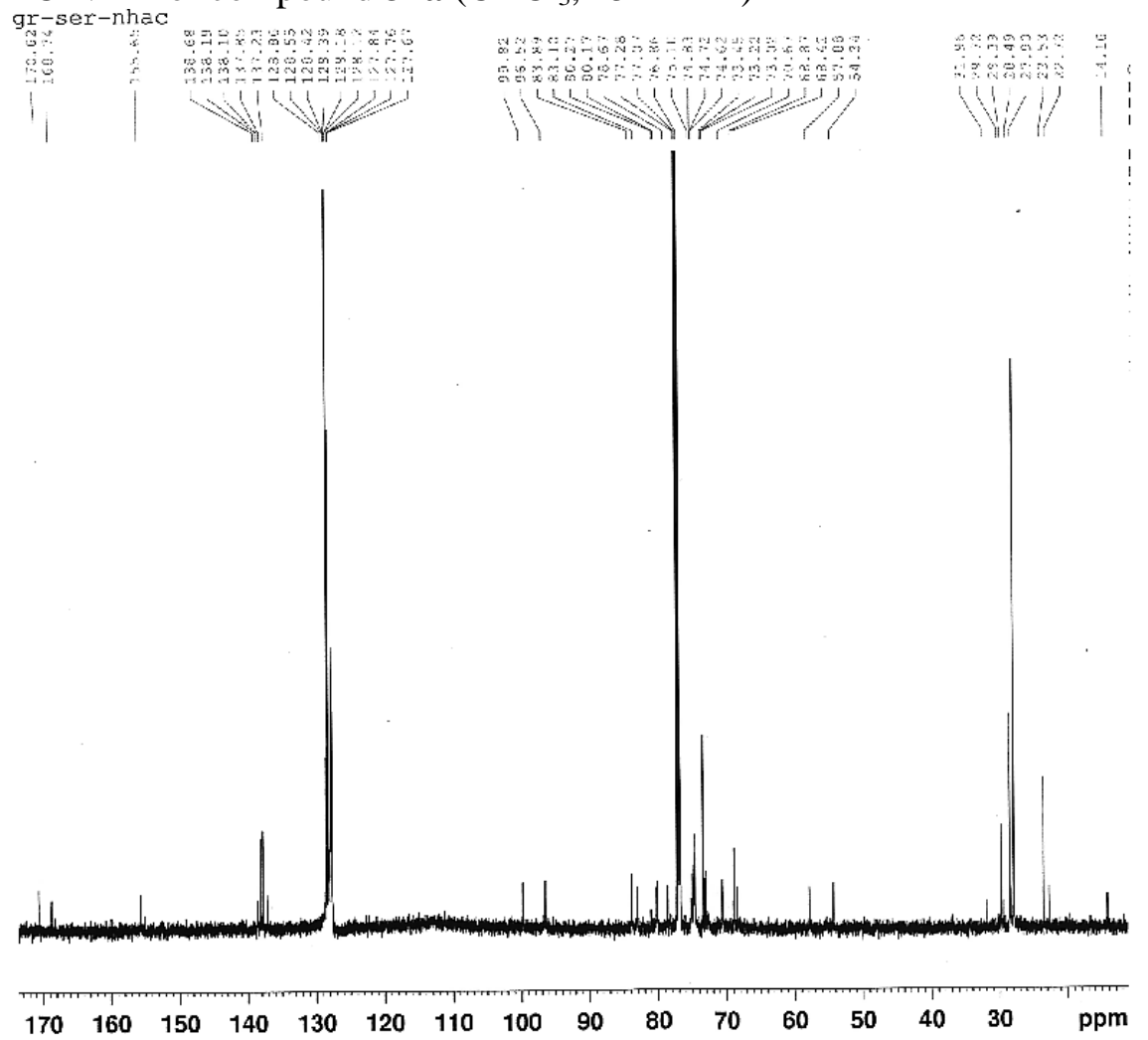

${ }^{1} \mathrm{H}$ NMR of compound 34b $\left(\mathrm{CDCl}_{3}, 600 \mathrm{MHz}\right)$ 


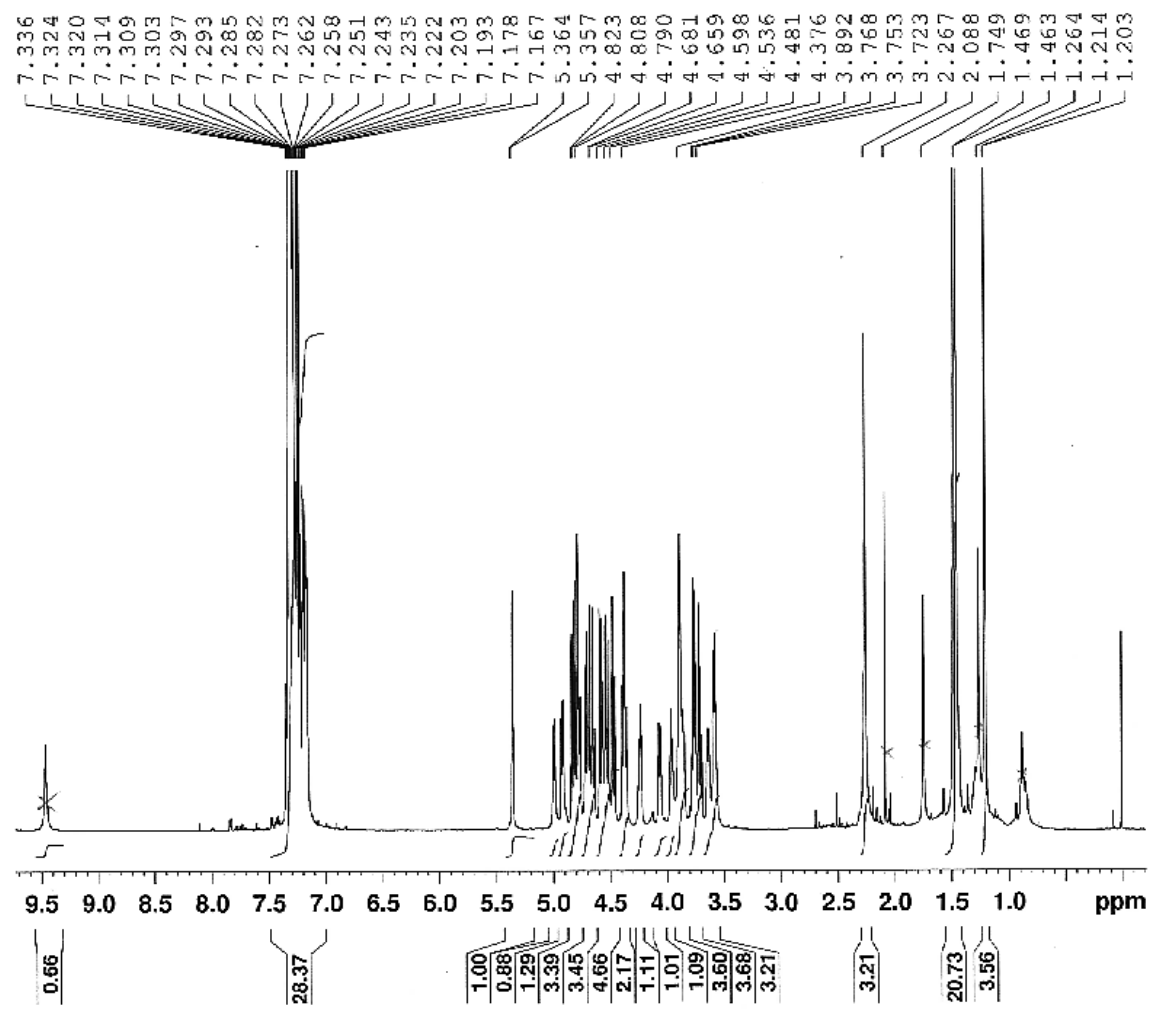

${ }^{13} \mathrm{C}$ NMR of compound $\mathbf{3 4 b}\left(\mathrm{CDCl}_{3}, 151 \mathrm{MHz}\right)$

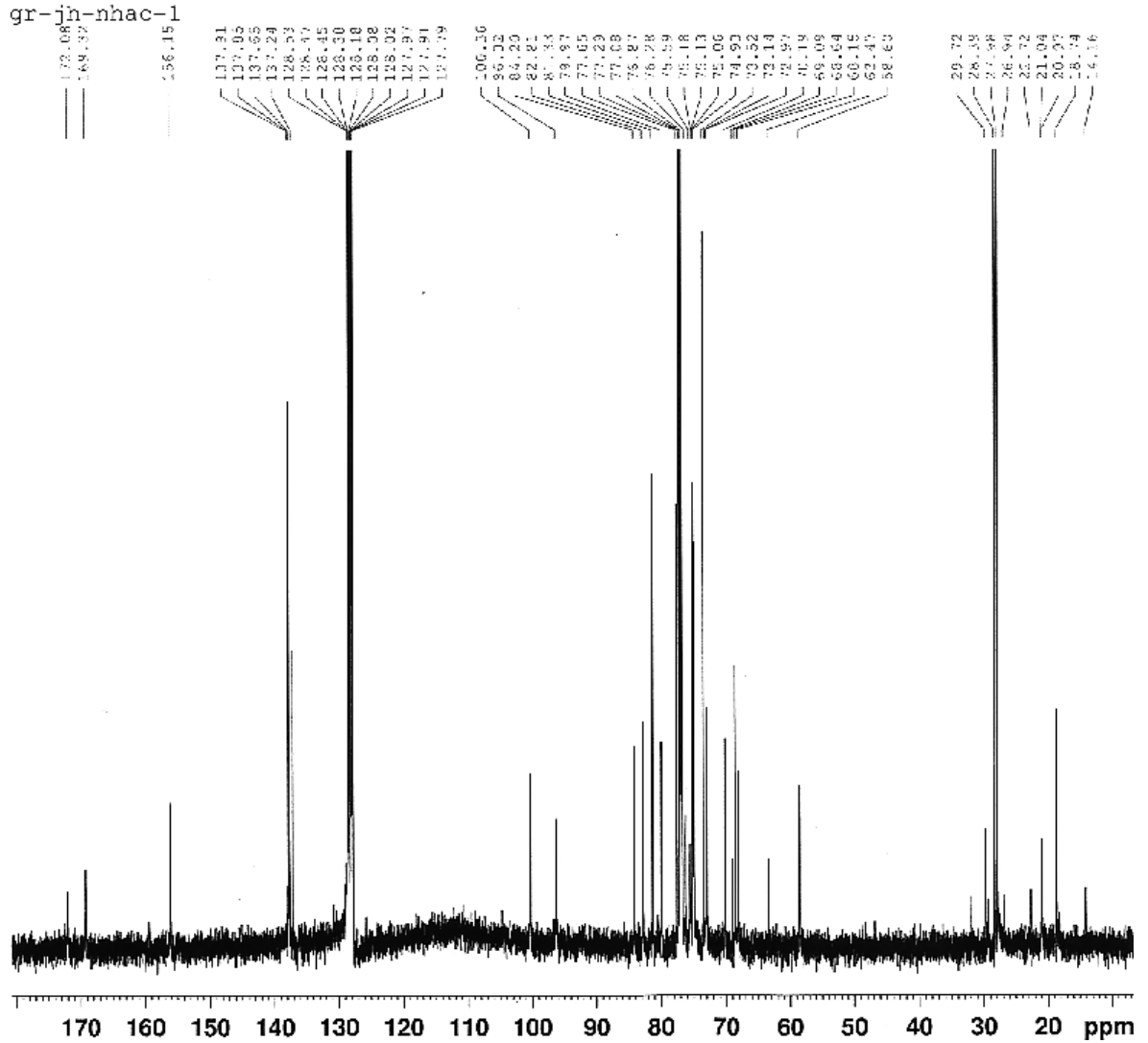

${ }^{1} \mathrm{H} \mathrm{NMR}$ of compound 35a $\left(\mathrm{CDCl}_{3}, 600 \mathrm{MHz}\right)$ 


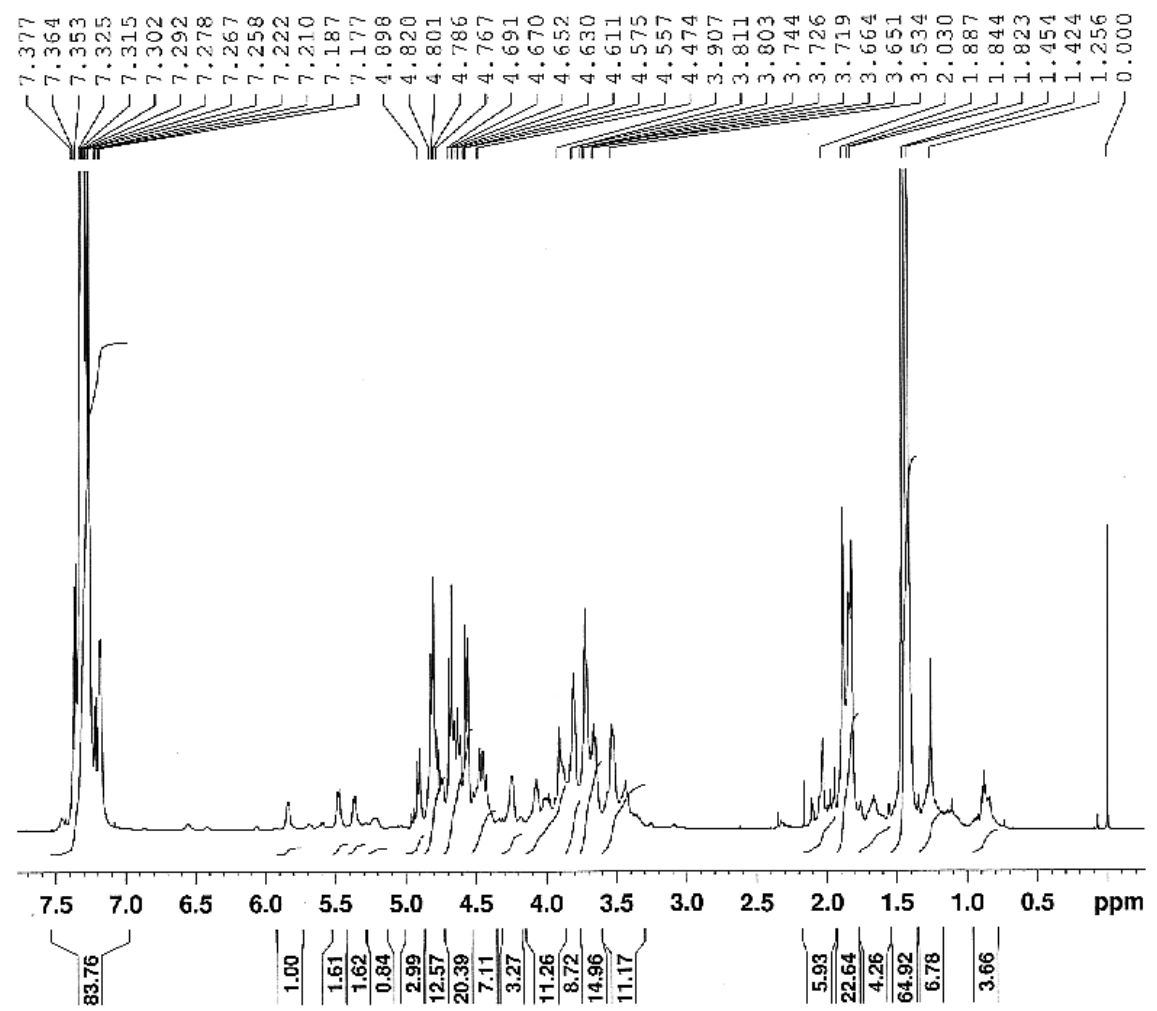

${ }^{13} \mathrm{C} \mathrm{NMR}$ of compound 35a $\left(\mathrm{CDCl}_{3}, 151 \mathrm{MHz}\right)$

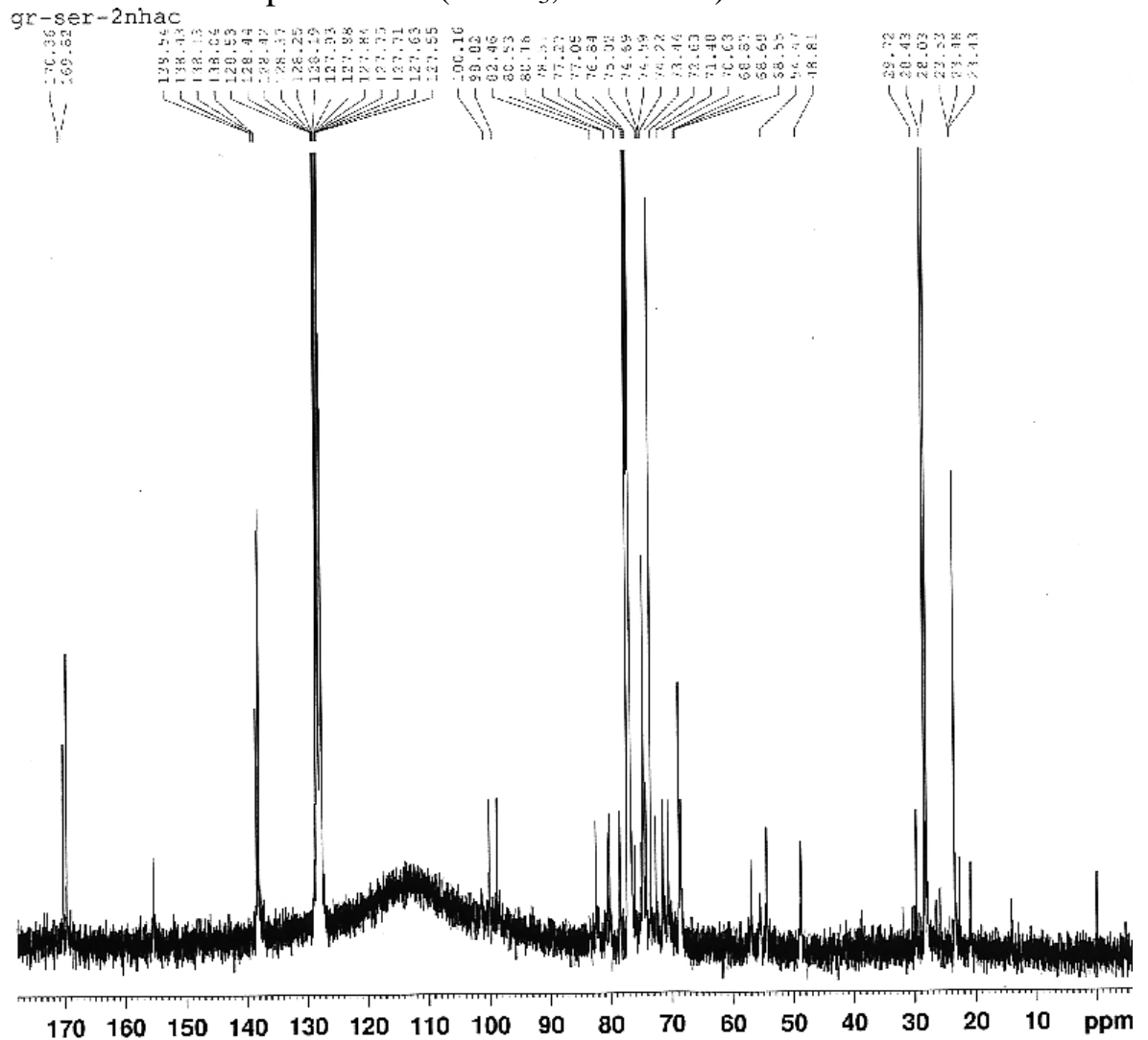

${ }^{1} \mathrm{H}$ NMR of compound $\mathbf{3 5 b}\left(\mathrm{CDCl}_{3}, 600 \mathrm{MHz}\right)$ 


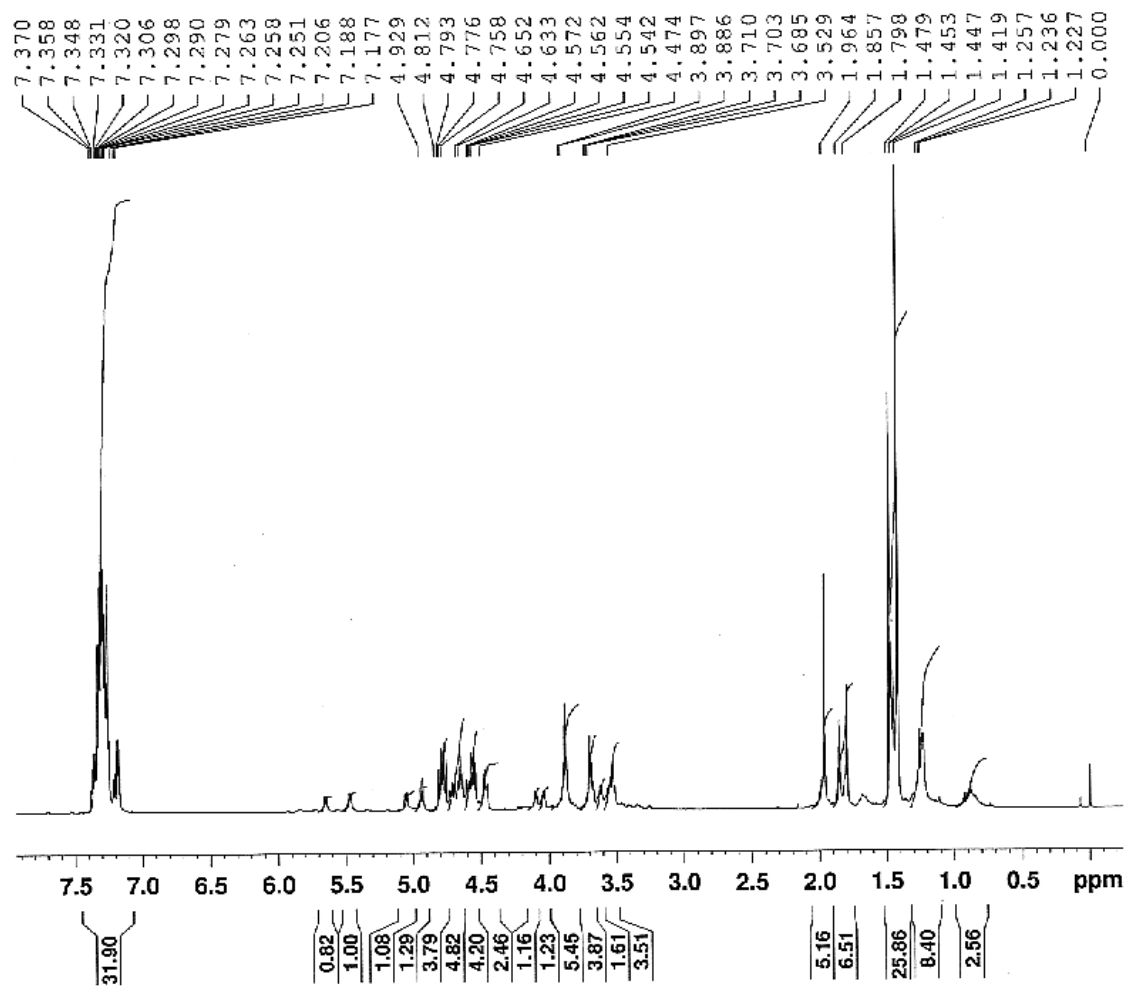

${ }^{13} \mathrm{C}$ NMR of compound $35 \mathrm{~b}\left(\mathrm{CDCl}_{3}, 151 \mathrm{MHz}\right)$

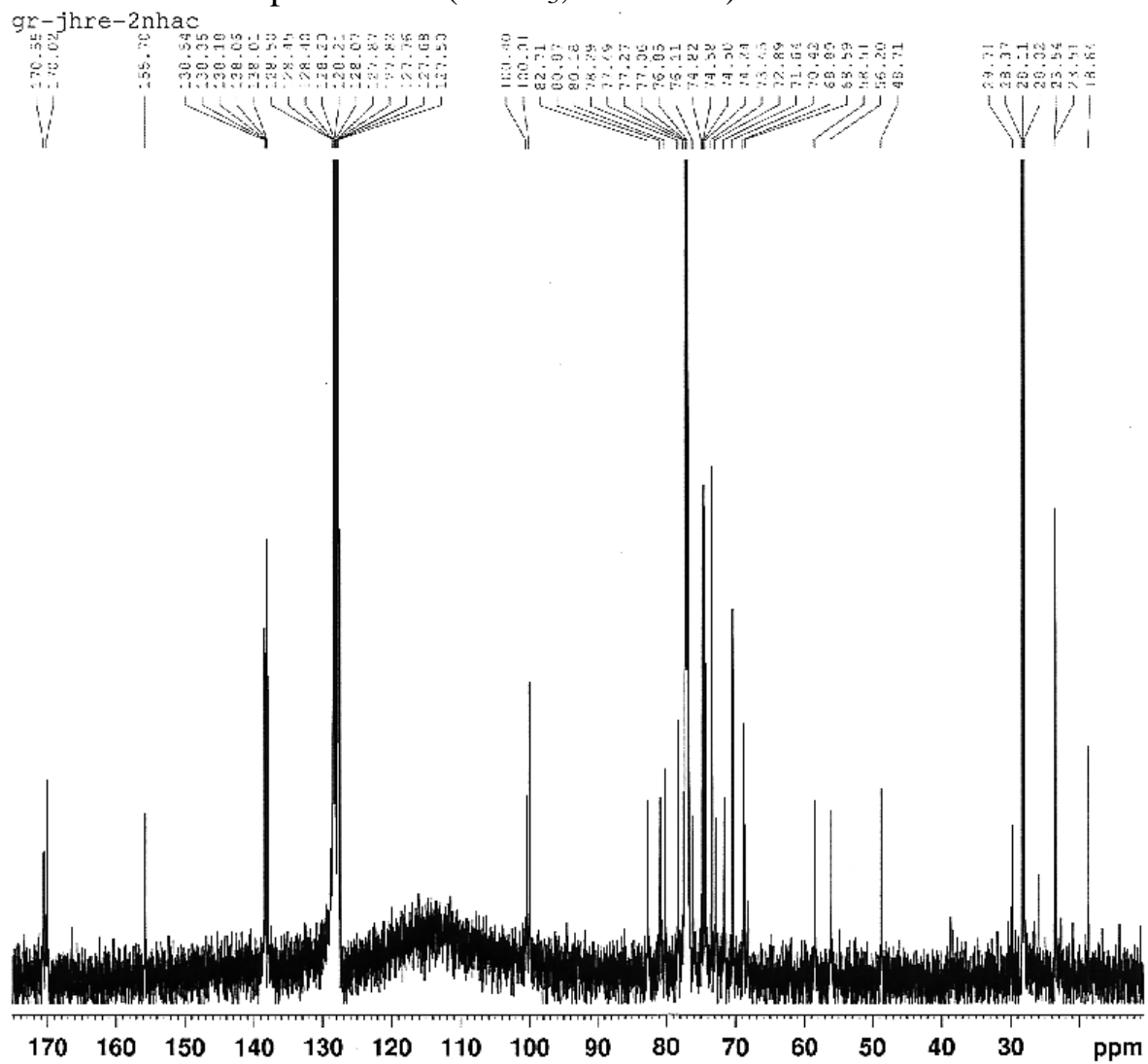

${ }^{1} \mathrm{H}$ NMR of compound $37\left(\mathrm{CDCl}_{3}, 600 \mathrm{MHz}\right)$ 


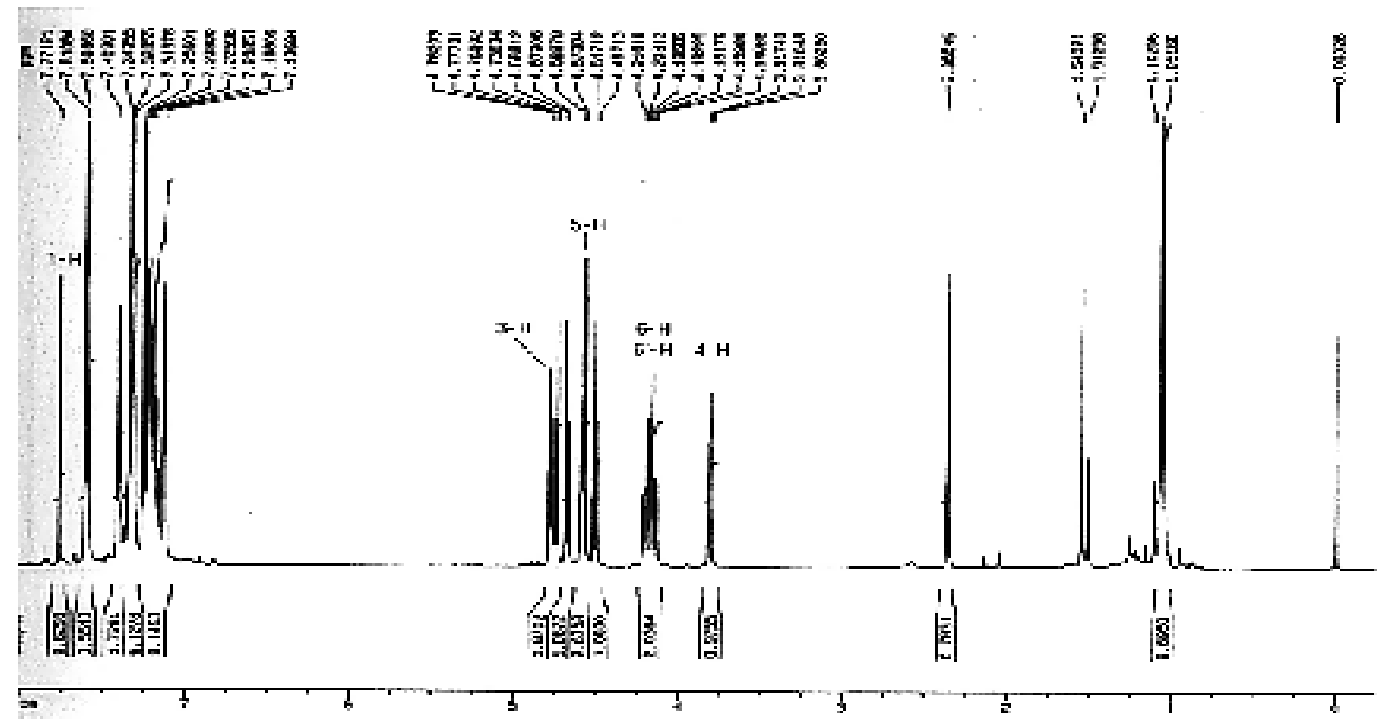

${ }^{13} \mathrm{C}$ NMR of compound $37\left(\mathrm{CDCl}_{3}, 151 \mathrm{MHz}\right)$

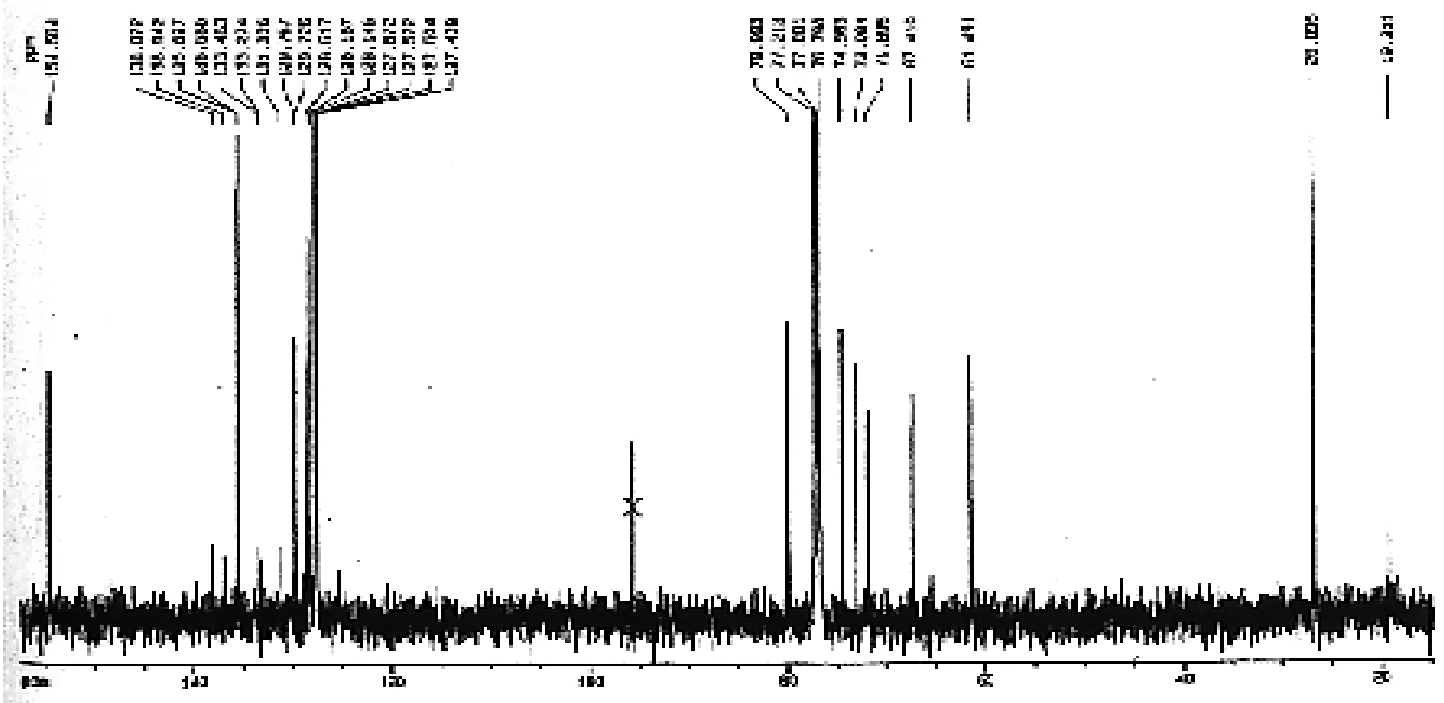




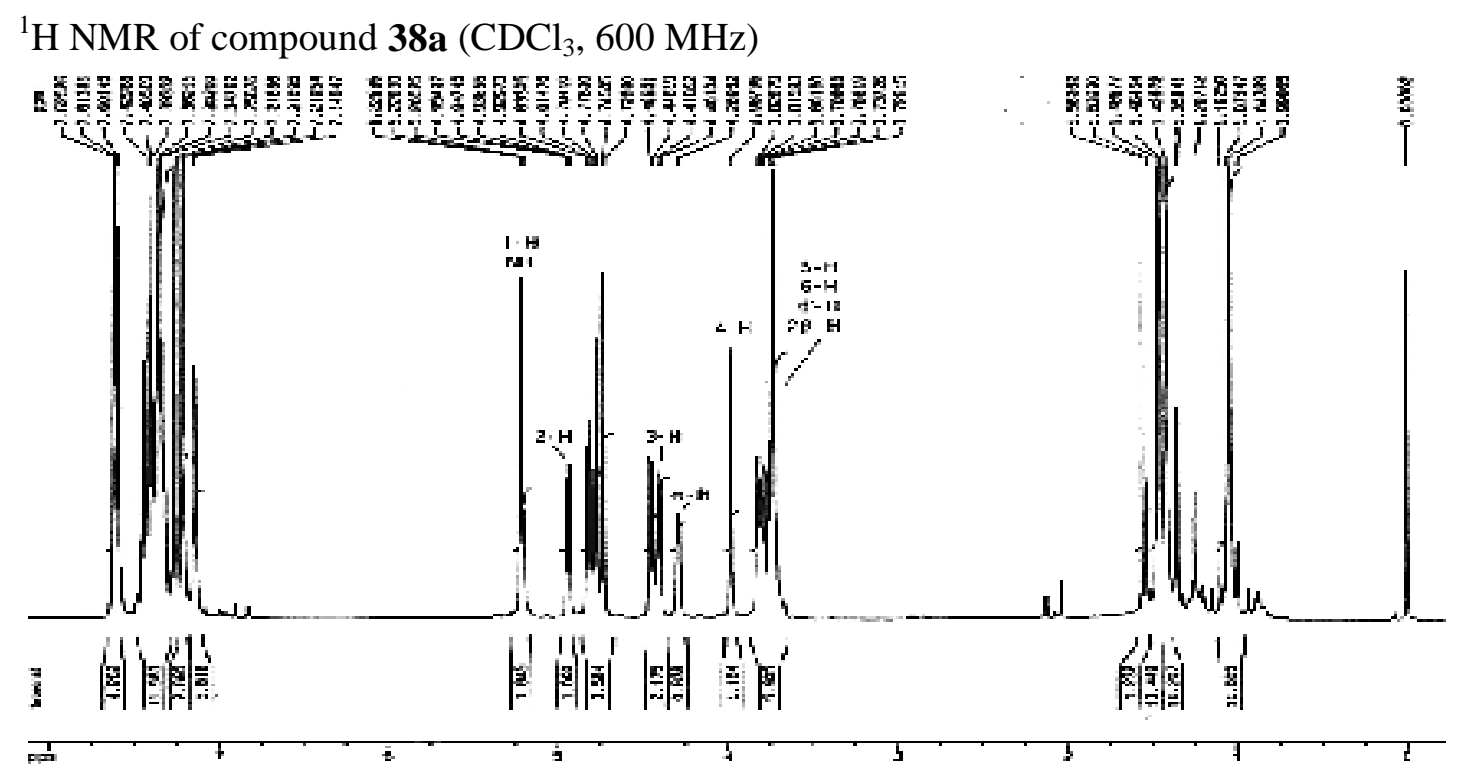

${ }^{13} \mathrm{C}$ NMR of compound 38a $\left(\mathrm{CDCl}_{3}, 151 \mathrm{MHz}\right)$

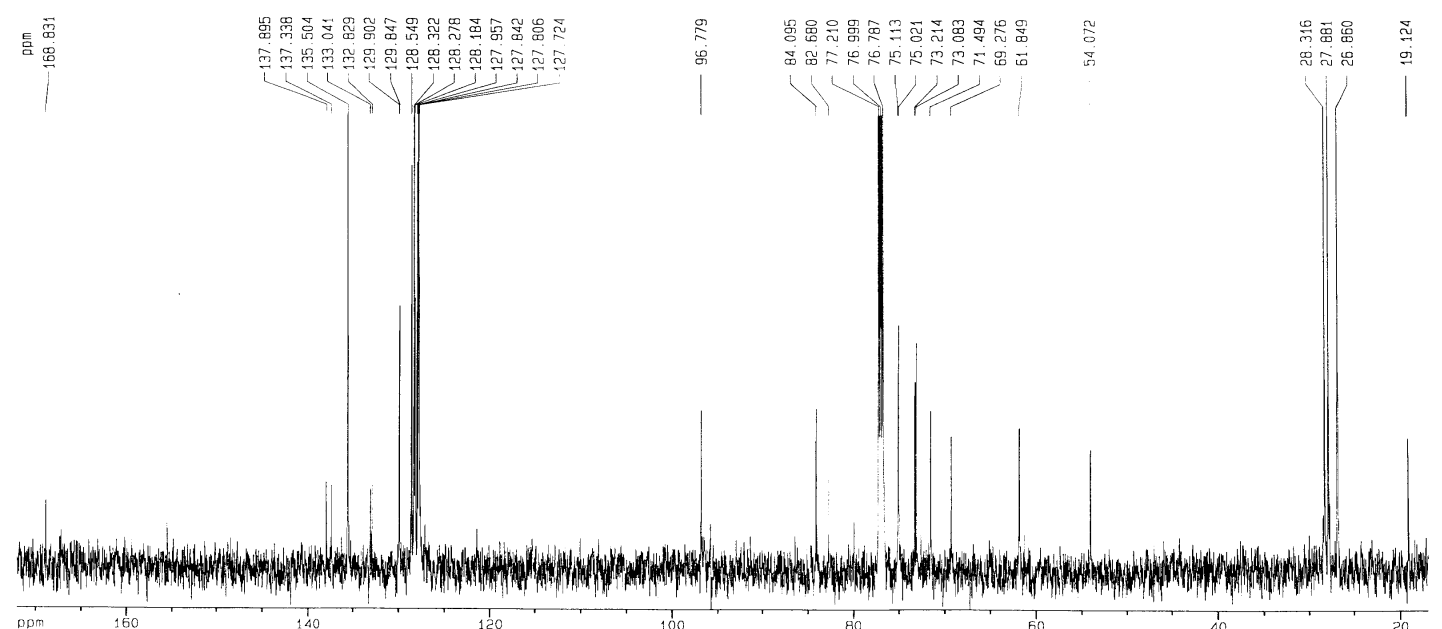




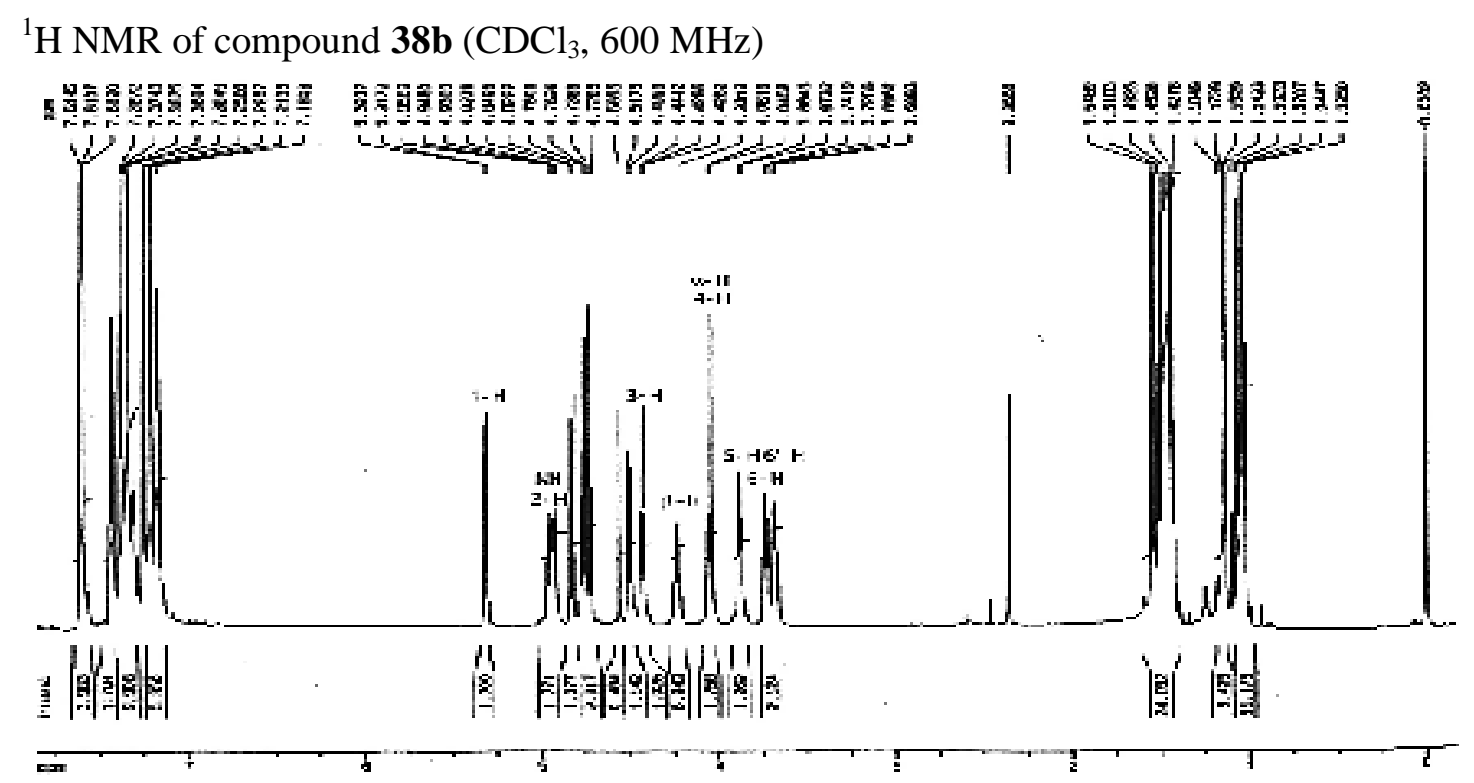

${ }^{13} \mathrm{C}$ NMR of compound $\mathbf{3 8 b}\left(\mathrm{CDCl}_{3}, 151 \mathrm{MHz}\right)$

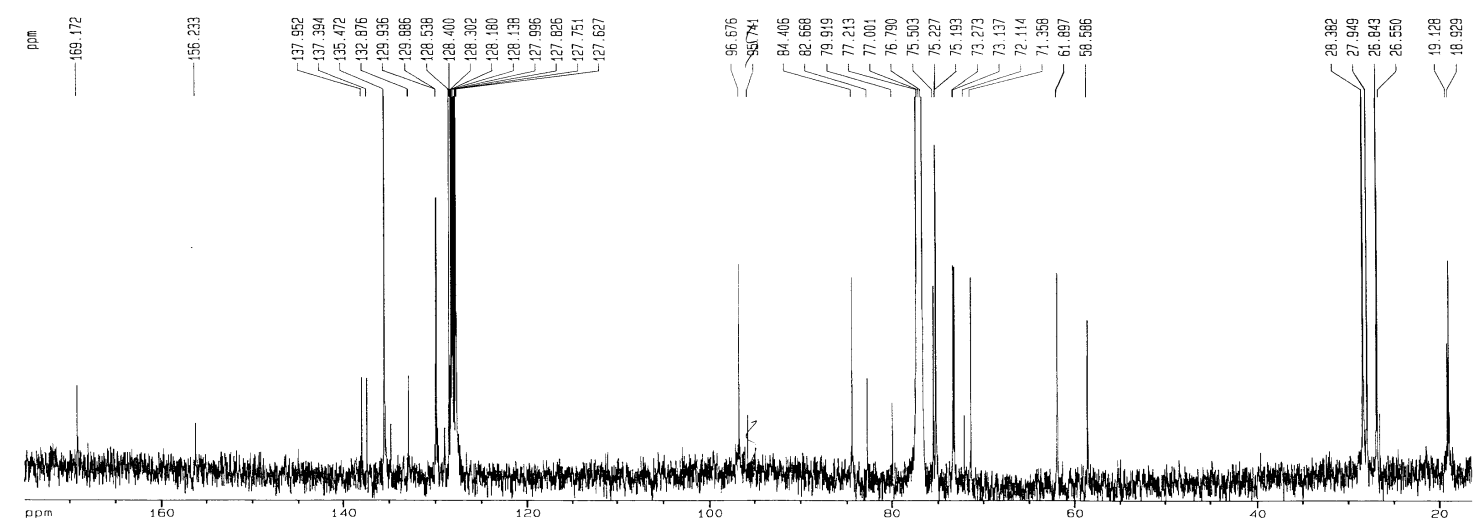


${ }^{11} \mathrm{H}$ NMR of compound 39a $\left(\mathrm{CDCl}_{3}, 600 \mathrm{MHz}\right)$

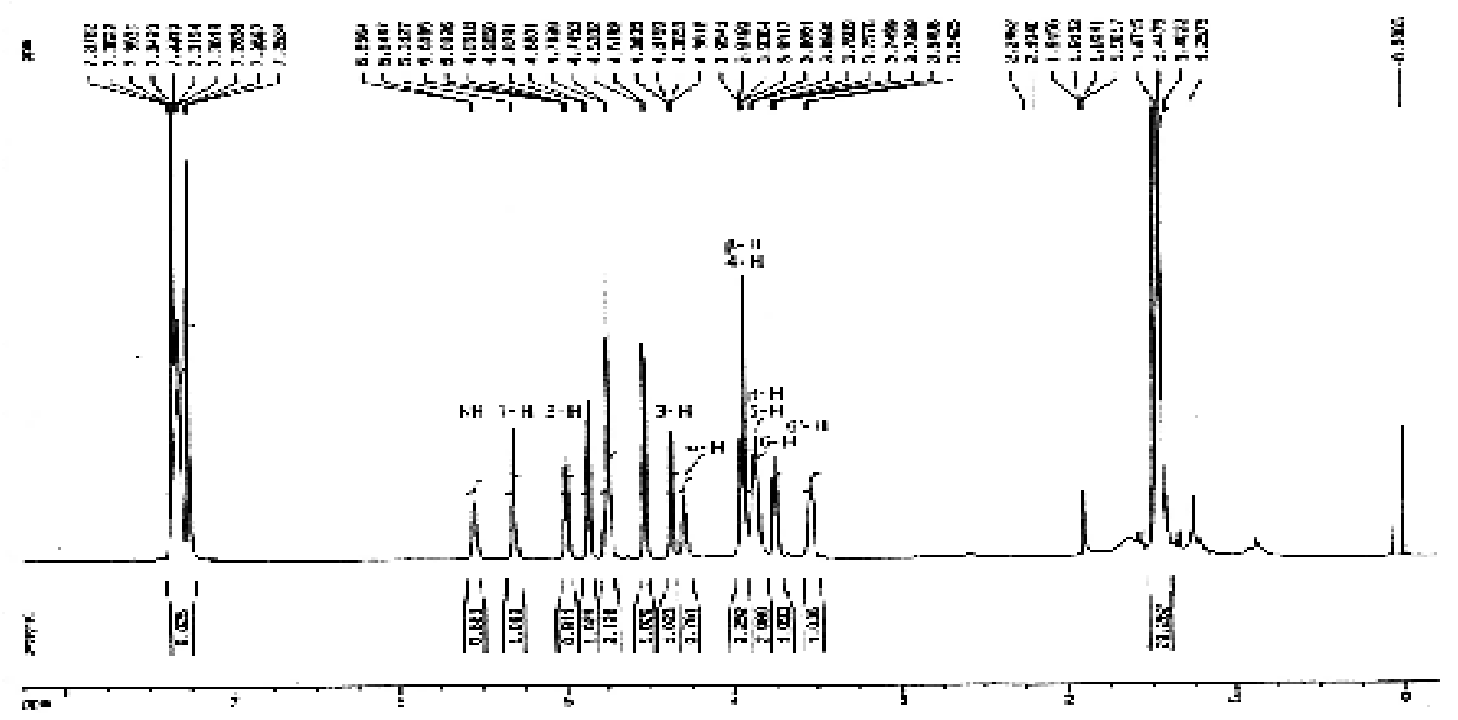

${ }^{13} \mathrm{C}$ NMR of compound 39a $\left(\mathrm{CDCl}_{3}, 151 \mathrm{MHz}\right)$

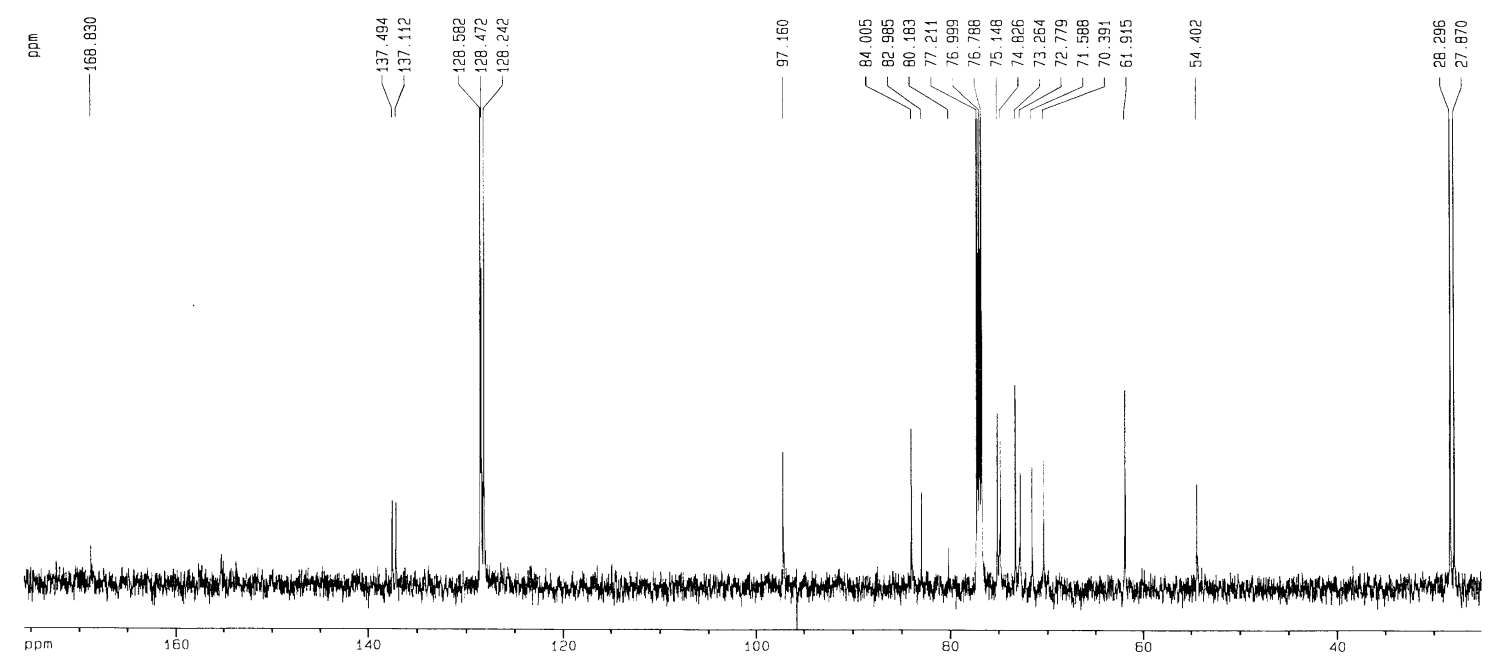




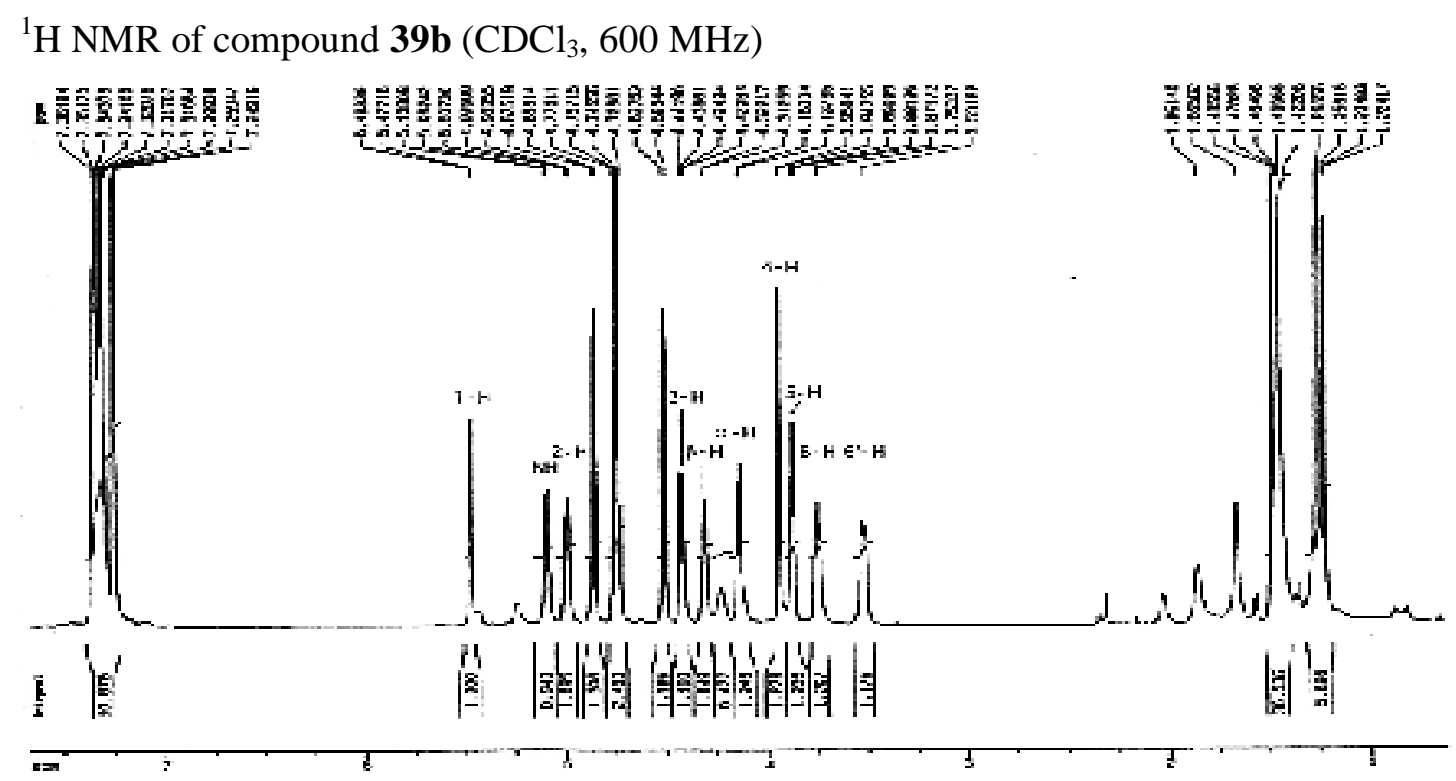

${ }^{13} \mathrm{C}$ NMR of compound 39b $\left(\mathrm{CDCl}_{3}, 151 \mathrm{MHz}\right)$

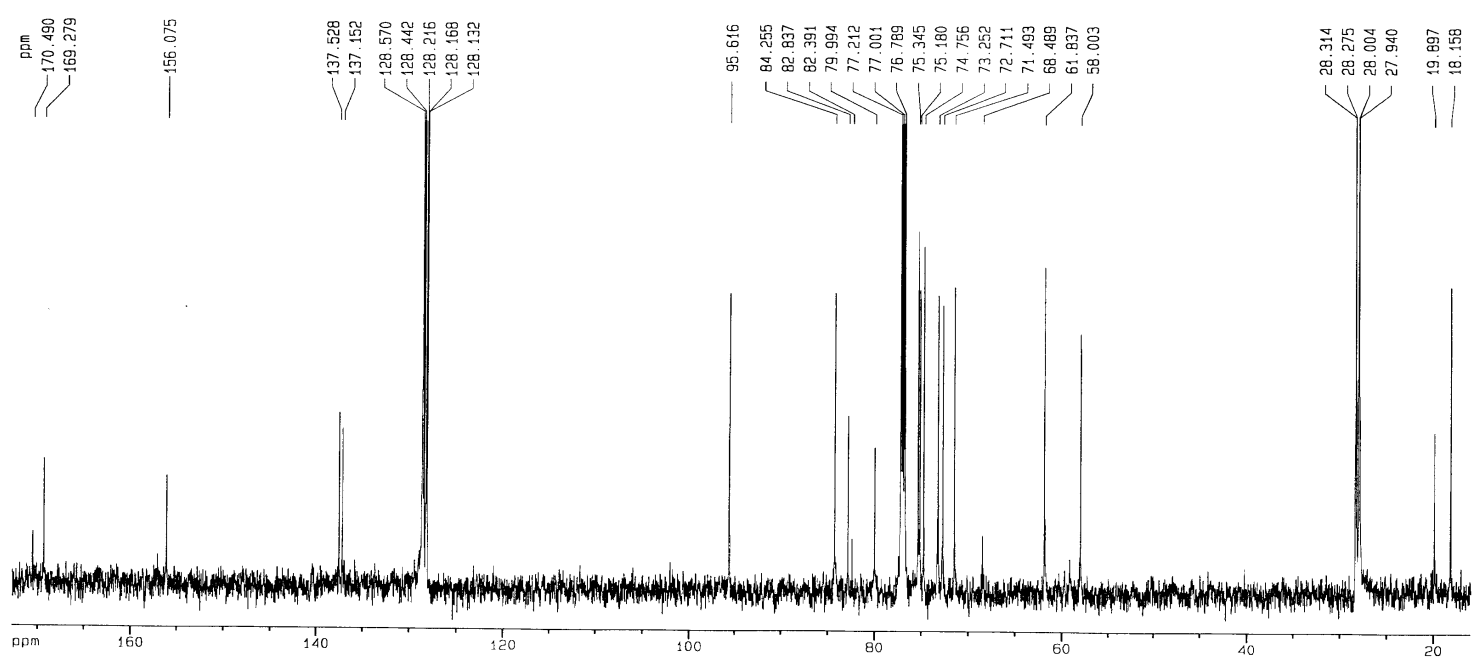




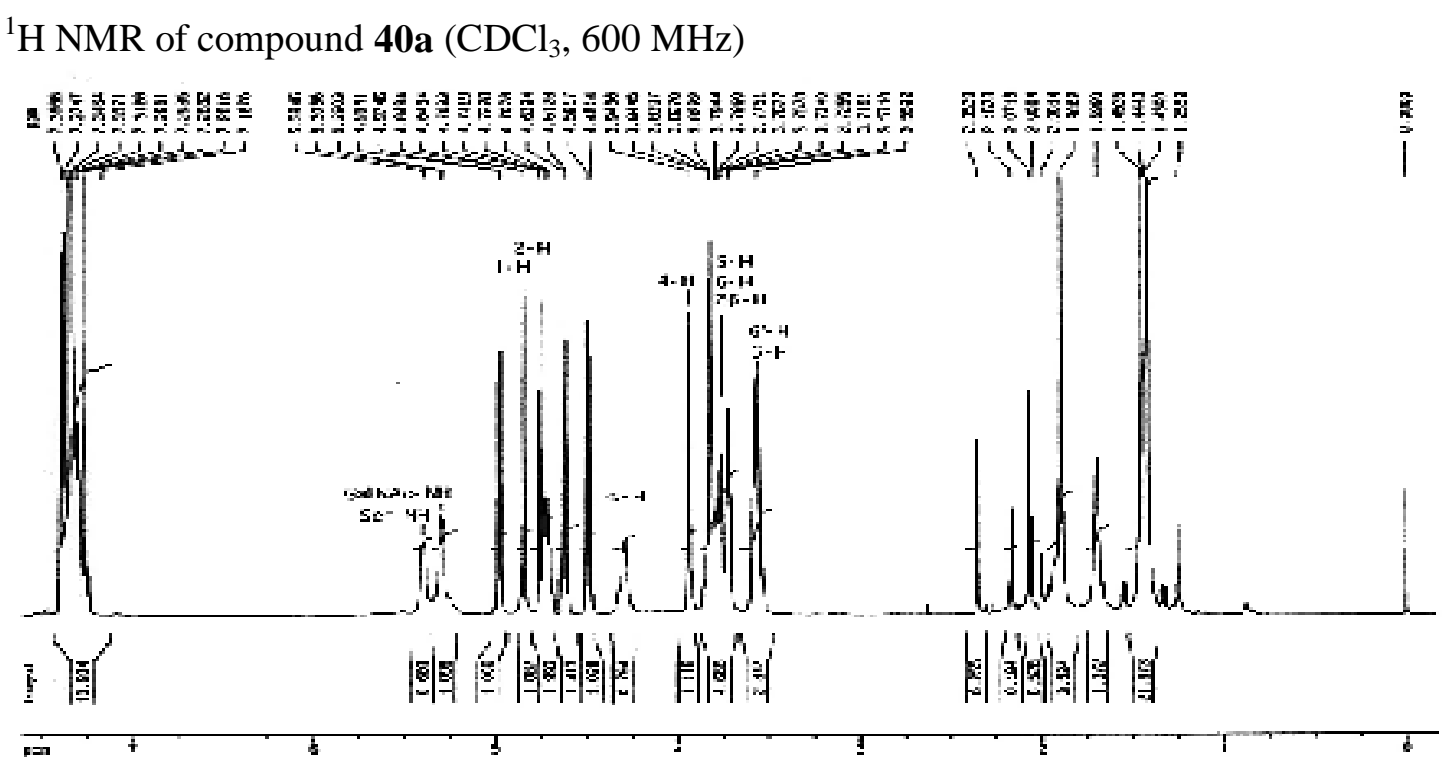

${ }^{13} \mathrm{C}$ NMR of compound 40a $\left(\mathrm{CDCl}_{3}, 151 \mathrm{MHz}\right)$

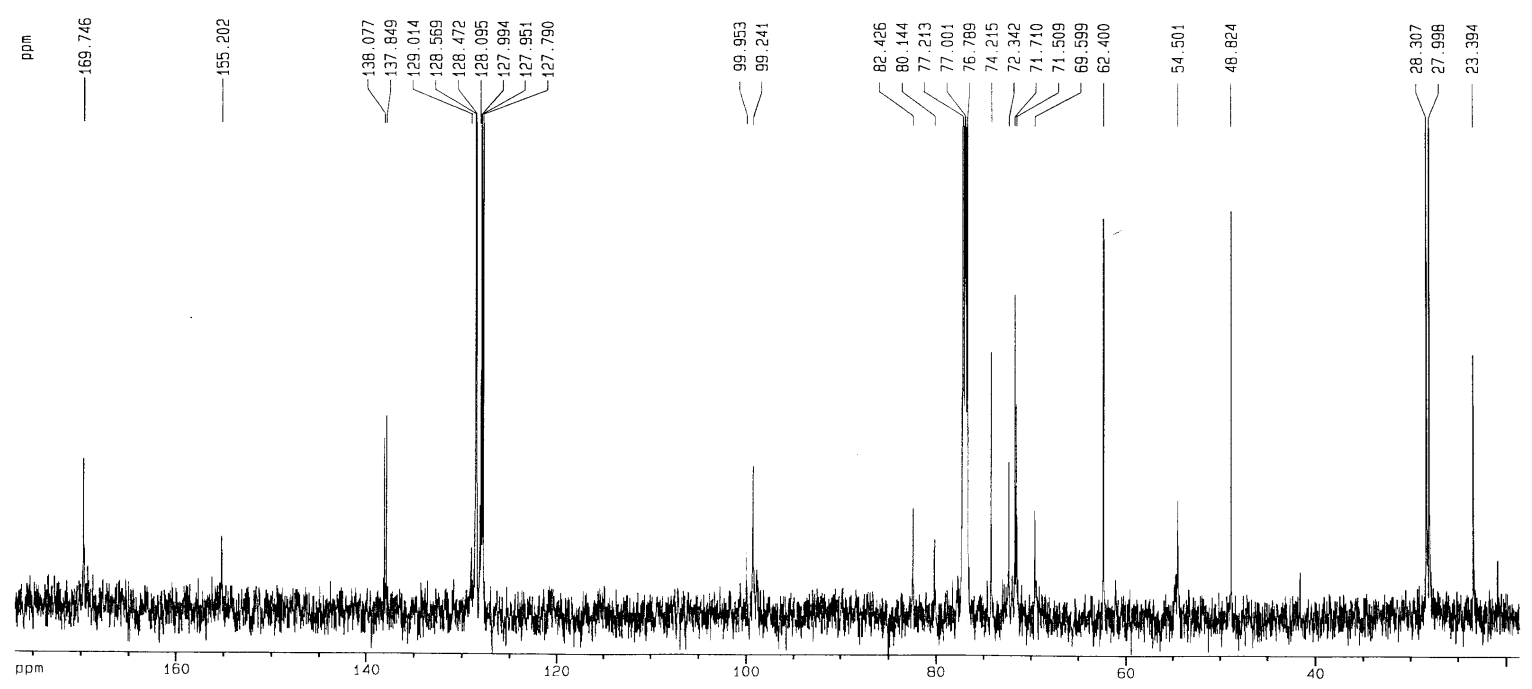




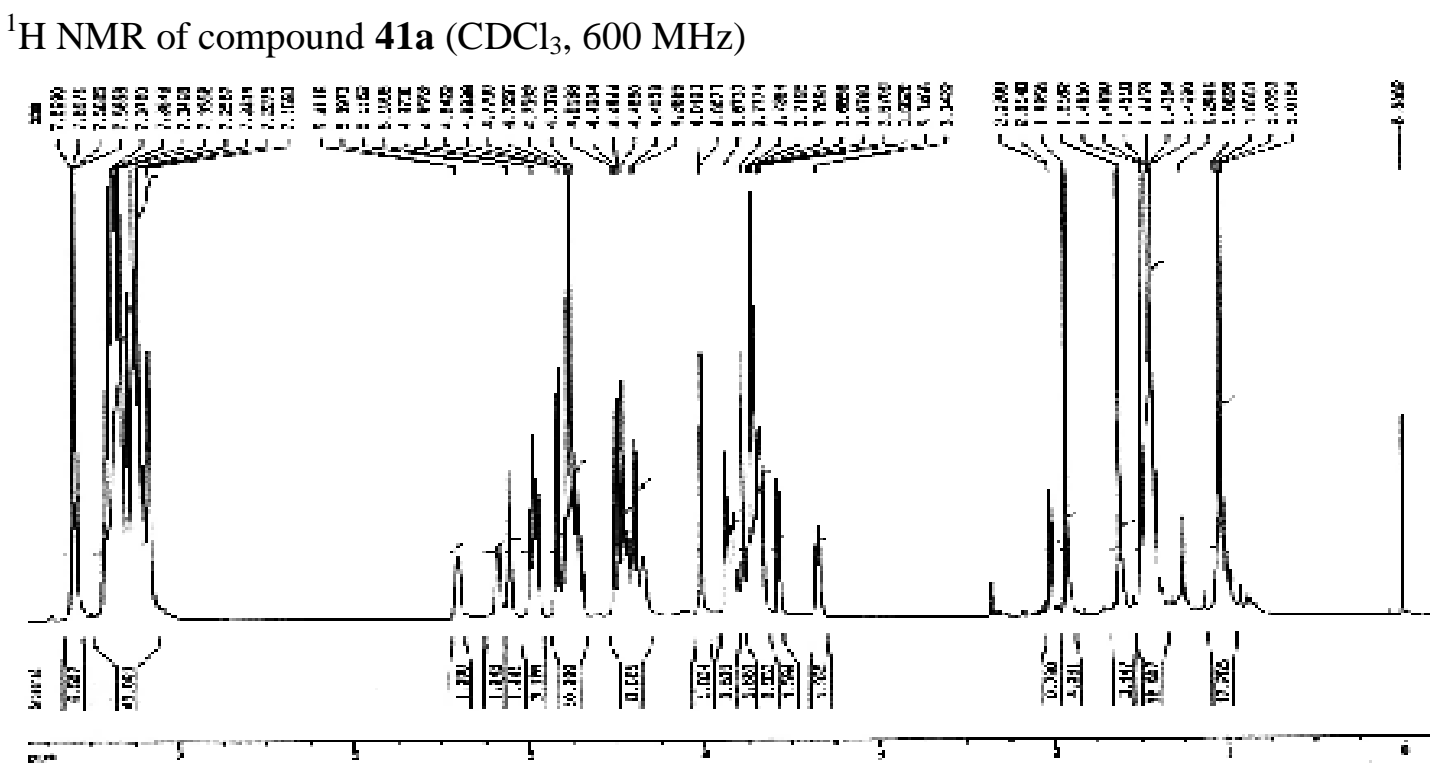

${ }^{13} \mathrm{C}$ NMR of compound 41a $\left(\mathrm{CDCl}_{3}, 151 \mathrm{MHz}\right)$

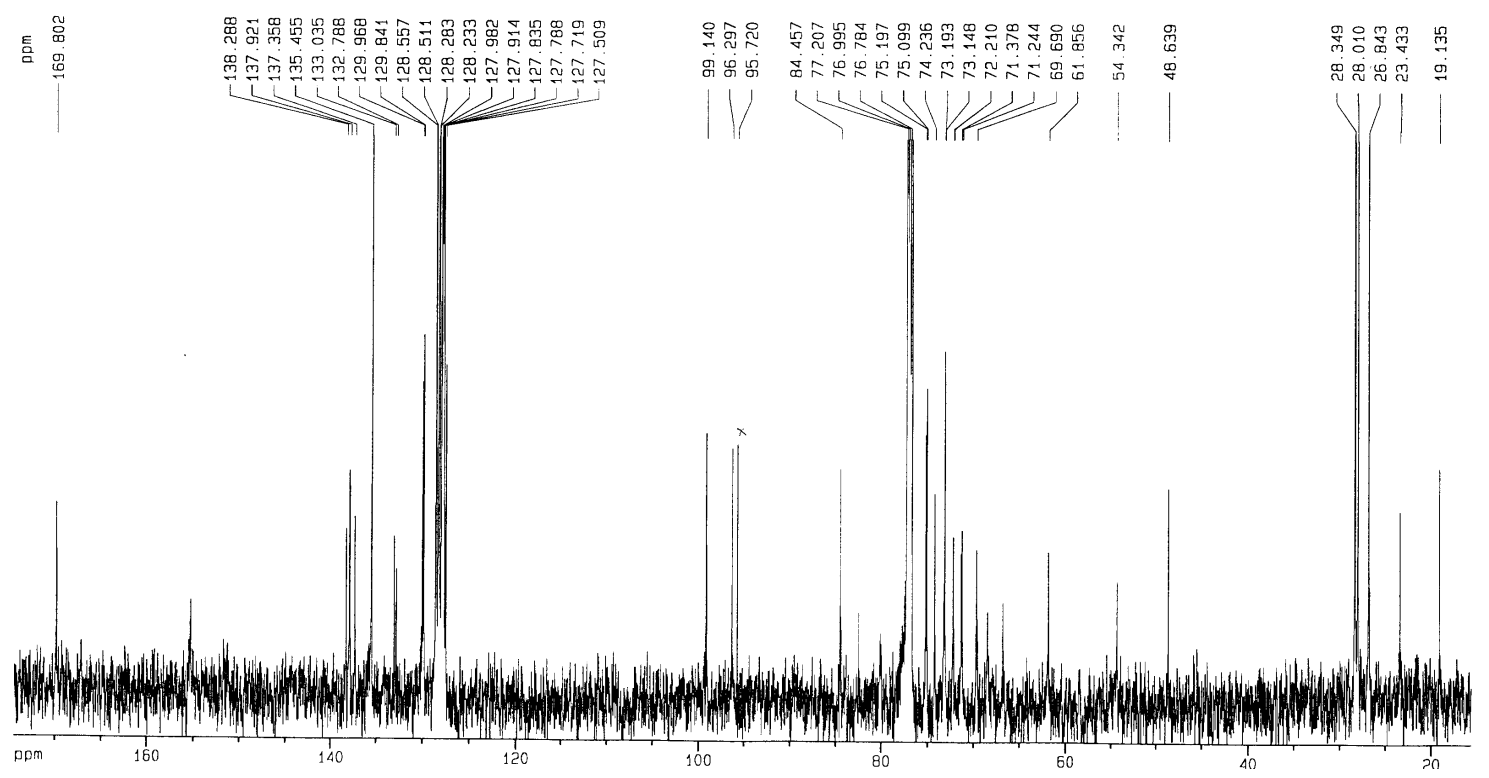




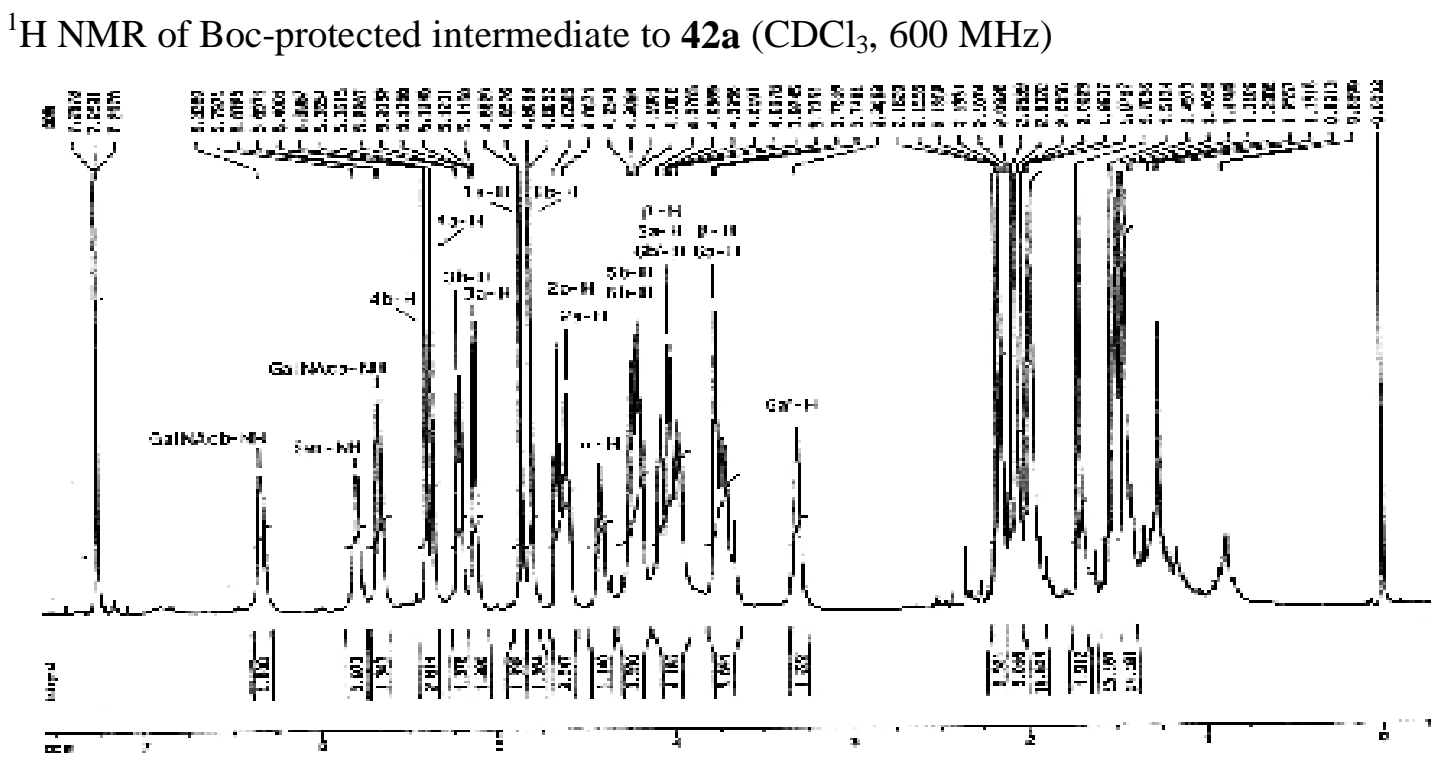

${ }^{1} \mathrm{H}$ NMR of compound 42a $\left(\mathrm{CDCl}_{3}, 600 \mathrm{MHz}\right)$

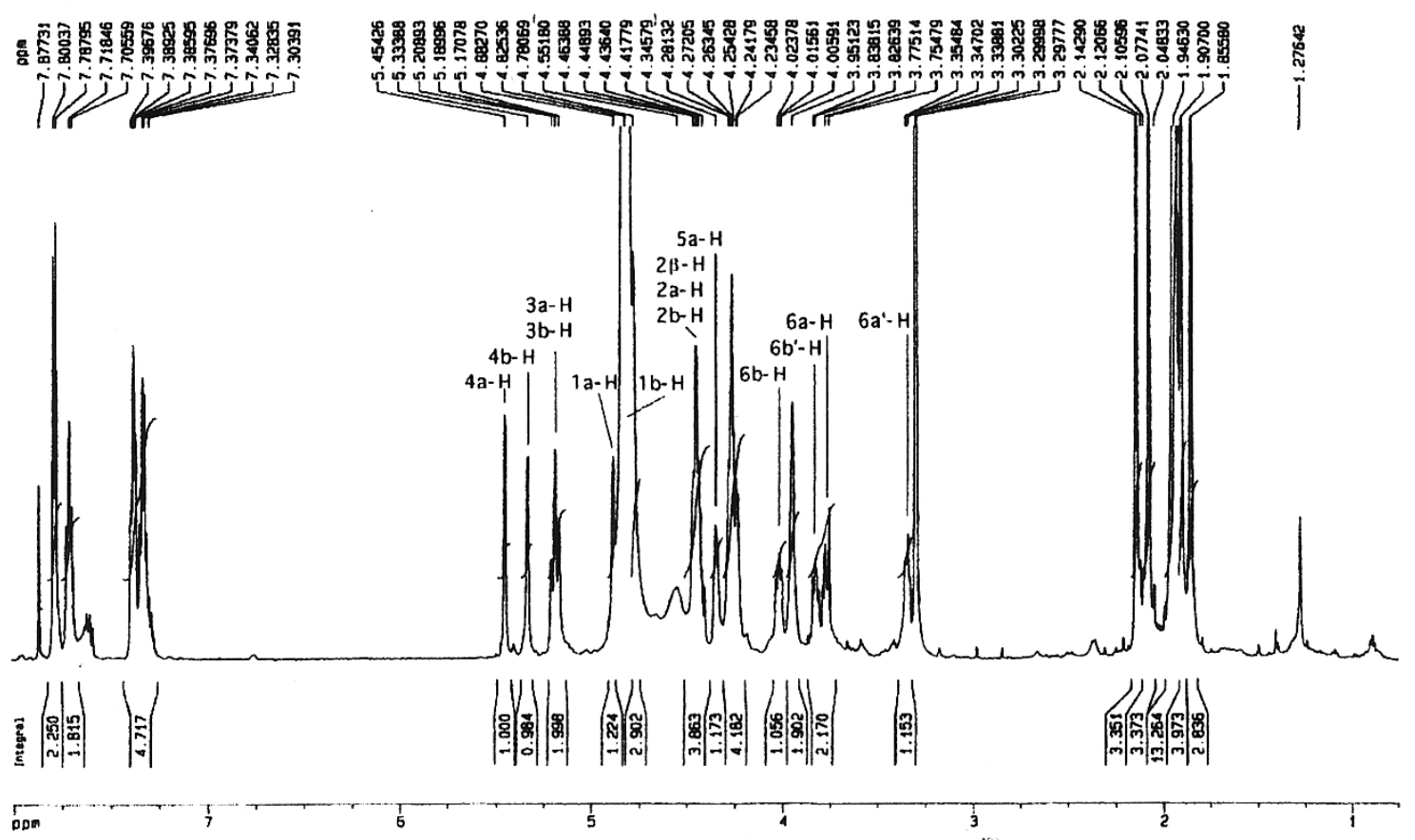


${ }^{13} \mathrm{C}$ NMR of compound $\mathbf{4 2 a}\left(\mathrm{CDCl}_{3}, 151 \mathrm{MHz}\right)$

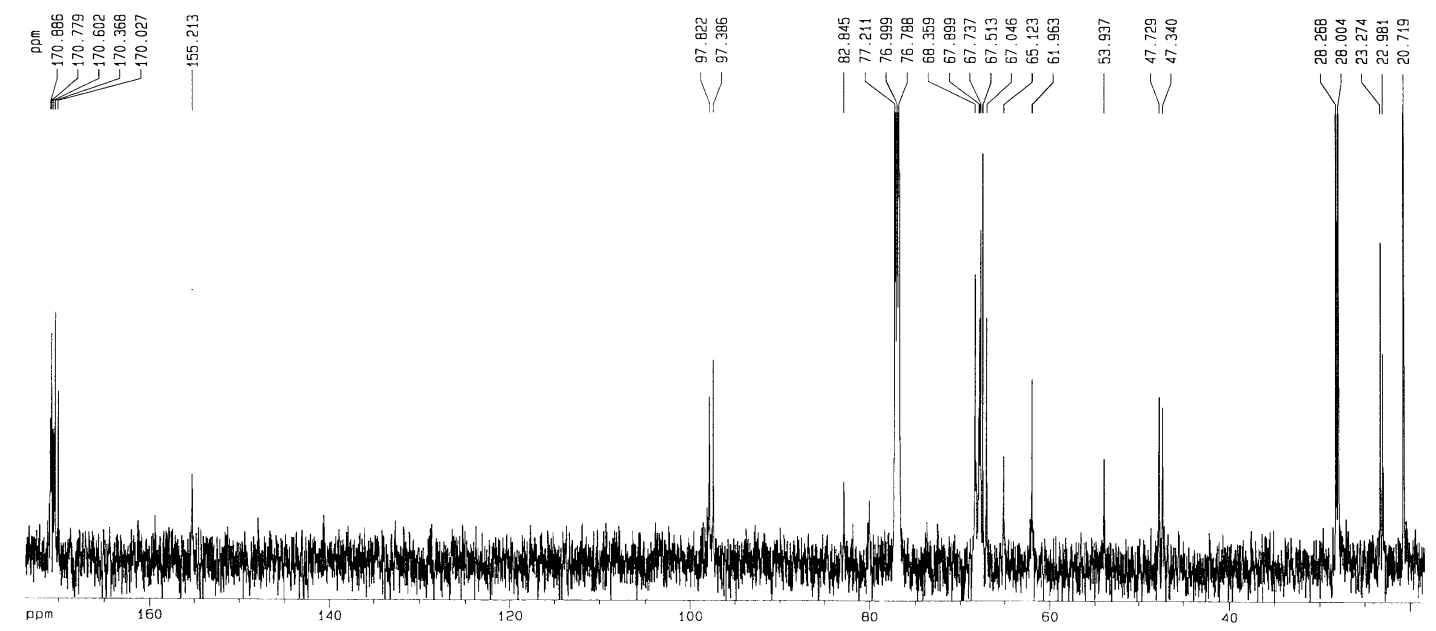

${ }^{1} \mathrm{H}$ NMR of compound $\mathbf{4 3}\left(\mathrm{CDCl}_{3}, 600 \mathrm{MHz}\right)$

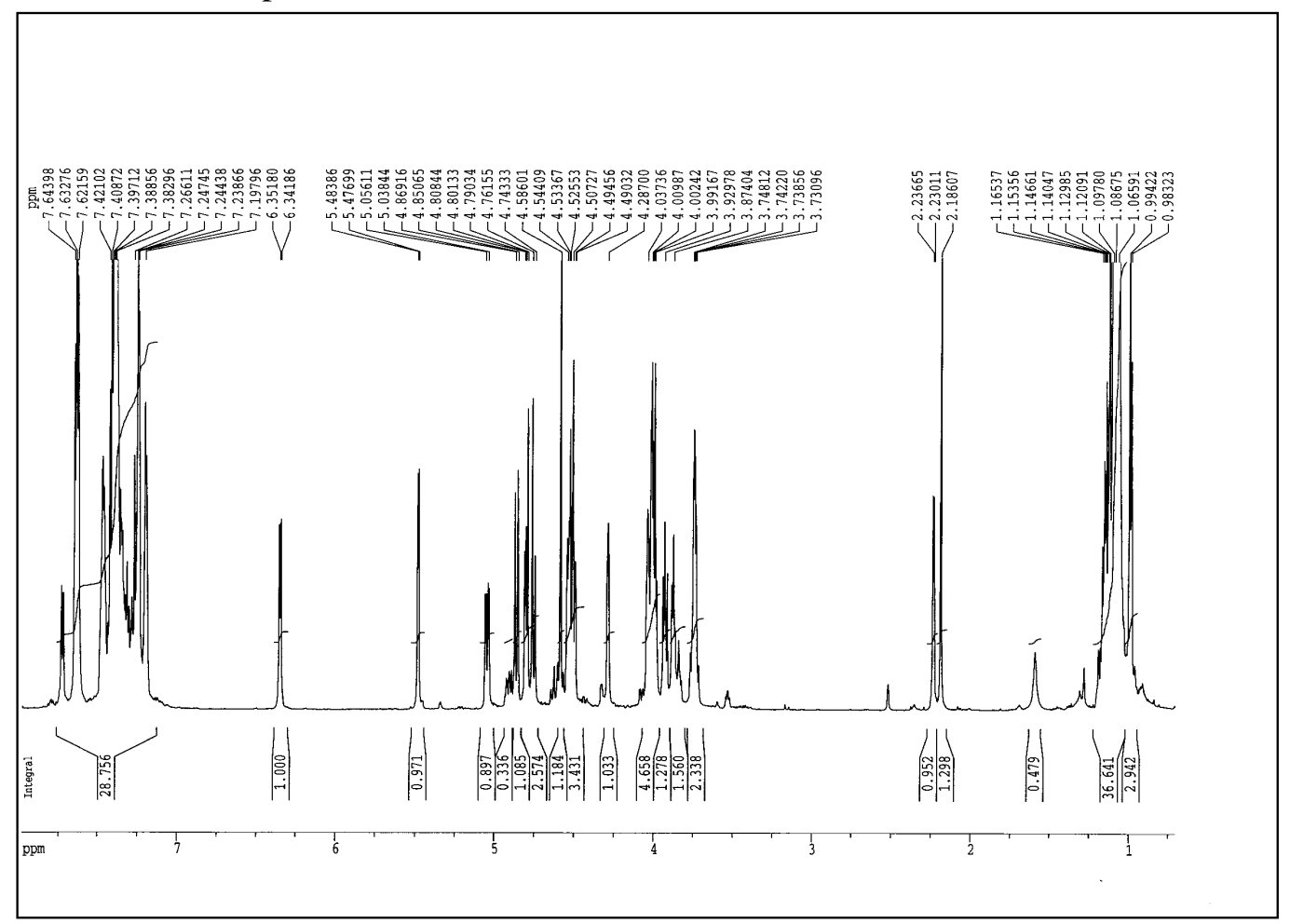


${ }^{13} \mathrm{C}$ NMR of compound $43\left(\mathrm{CDCl}_{3}, 151 \mathrm{MHz}\right)$

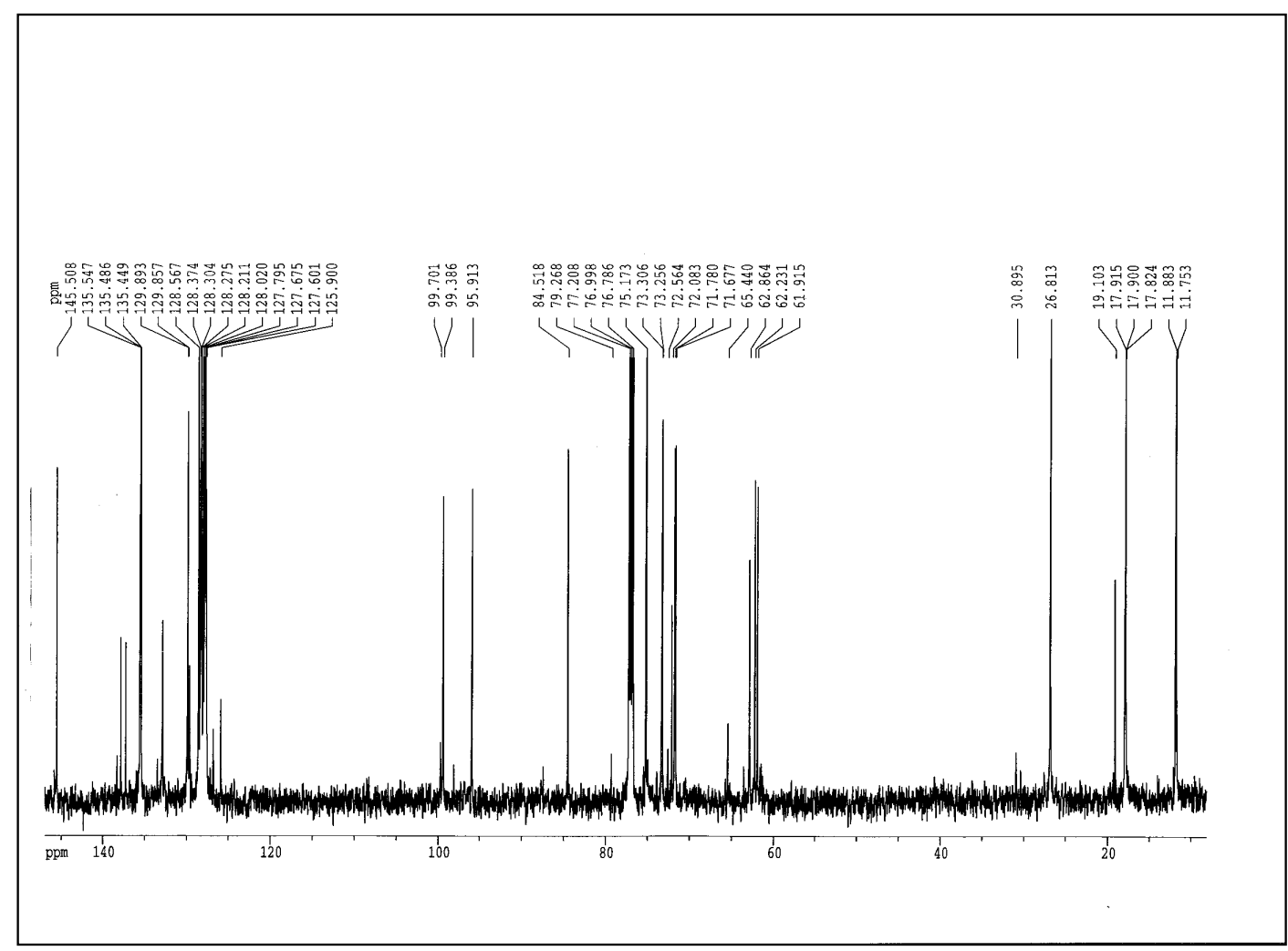

${ }^{1} \mathrm{H}$ NMR of compound $44\left(\mathrm{CDCl}_{3}, 250 \mathrm{MHz}\right)$

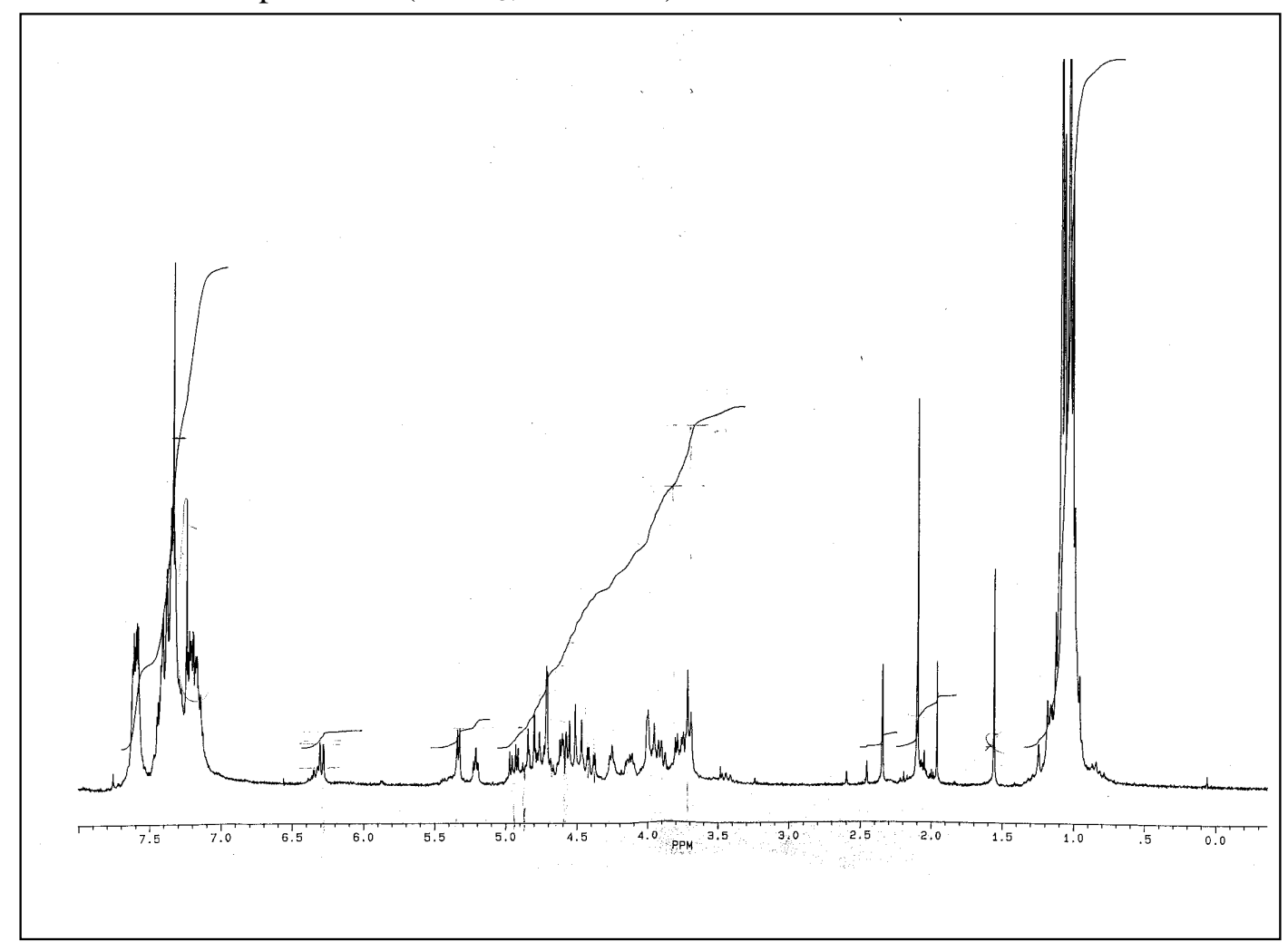


${ }^{13} \mathrm{C}$ NMR of compound $44\left(\mathrm{CDCl}_{3}, 151 \mathrm{MHz}\right)$

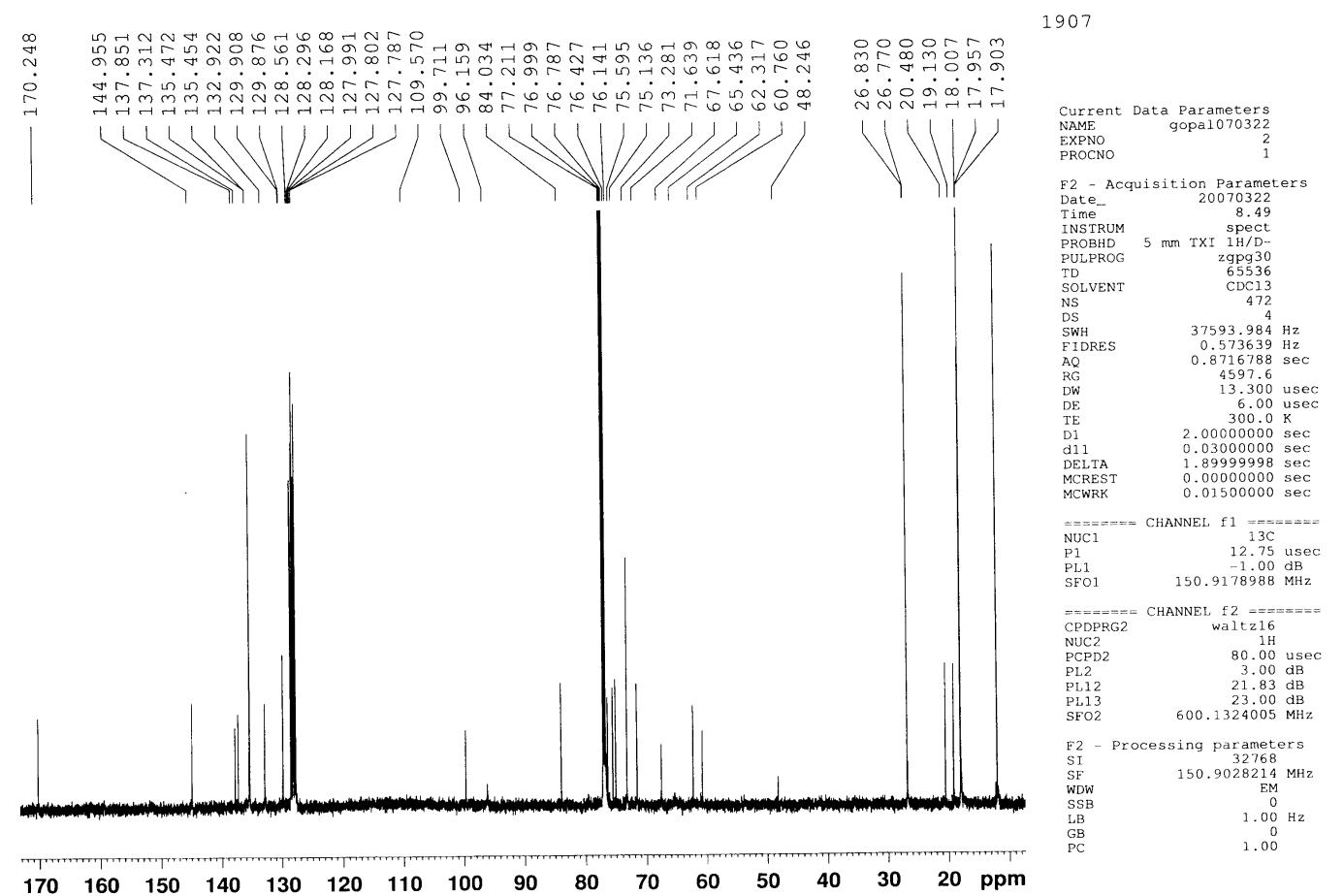

${ }^{1} \mathrm{H}$ NMR of compound $45\left(\mathrm{CDCl}_{3}, 600 \mathrm{MHz}\right)$

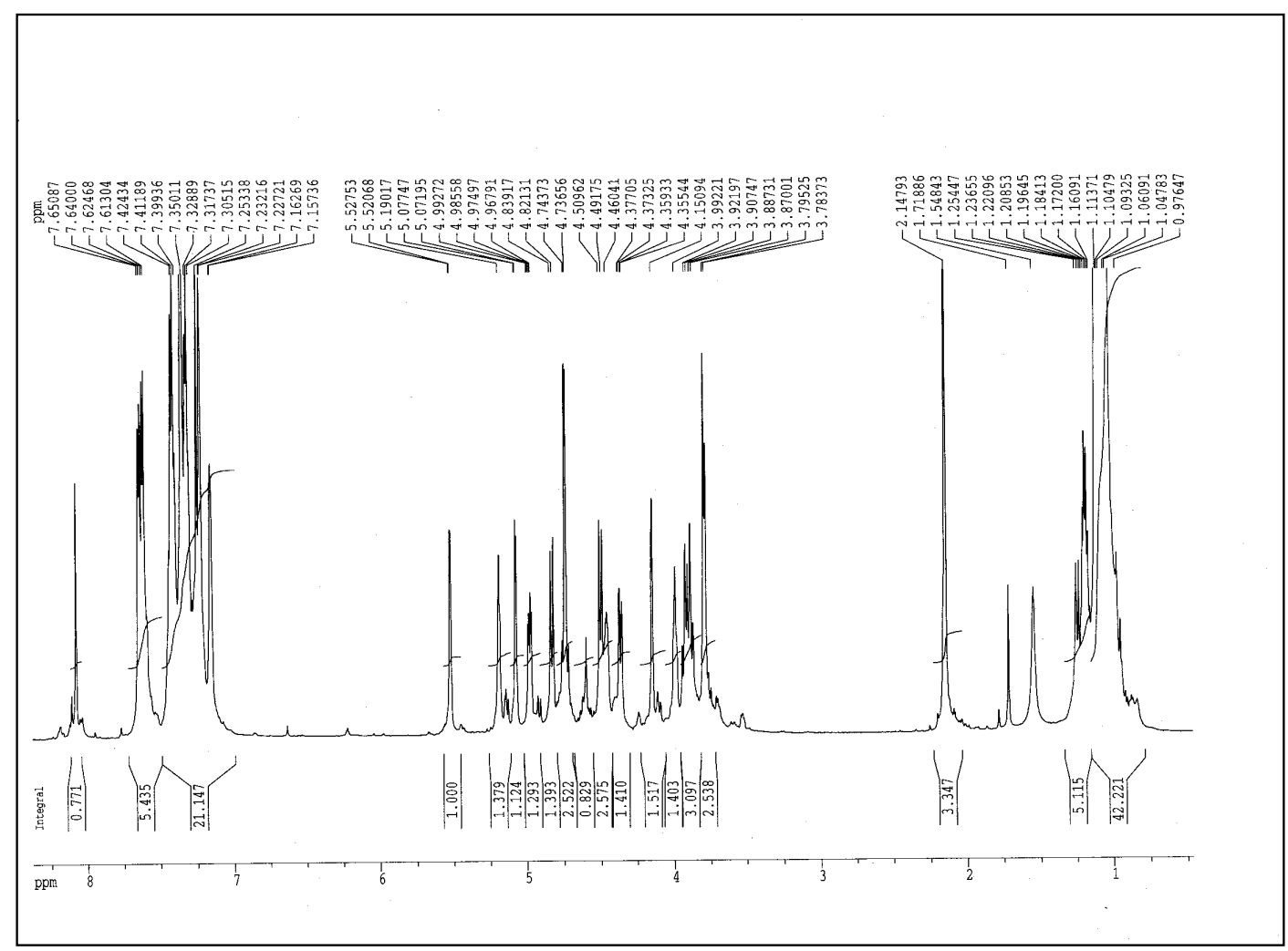


${ }^{13} \mathrm{C}$ NMR of compound $45\left(\mathrm{CDCl}_{3}, 151 \mathrm{MHz}\right)$

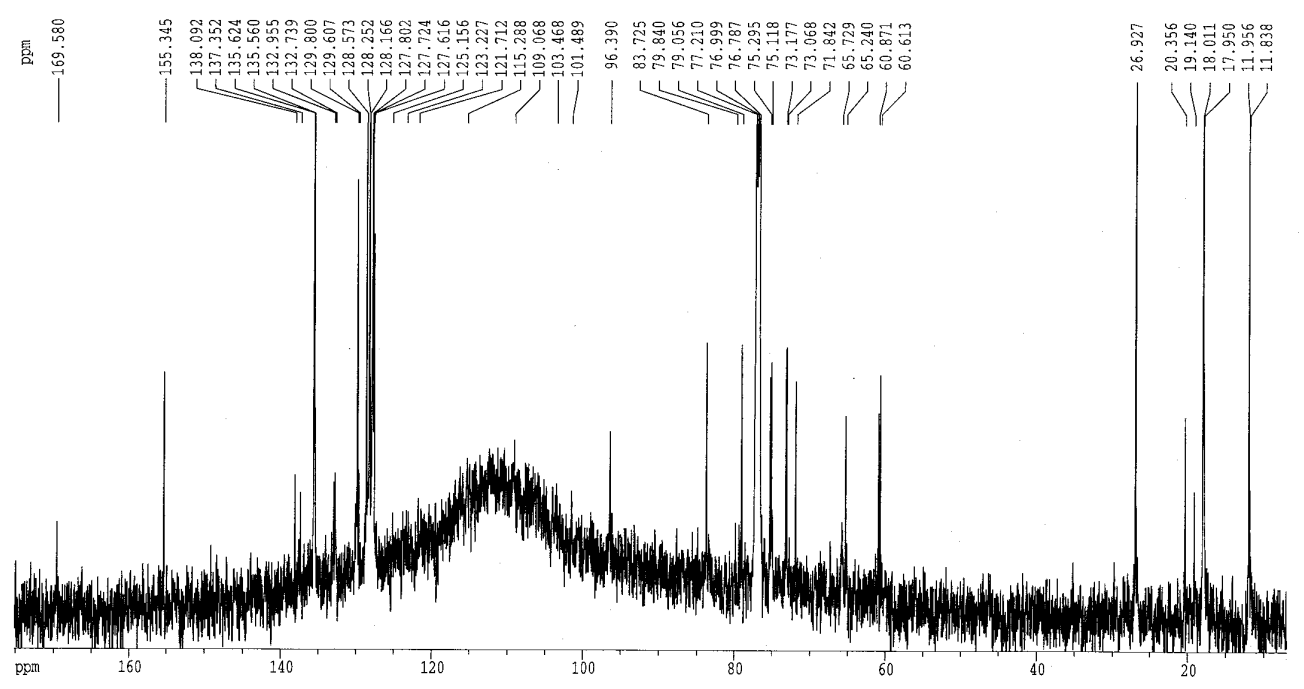

${ }^{1} \mathrm{H}$ NMR of compound 46a $\left(\mathrm{CDCl}_{3}, 600 \mathrm{MHz}\right)$

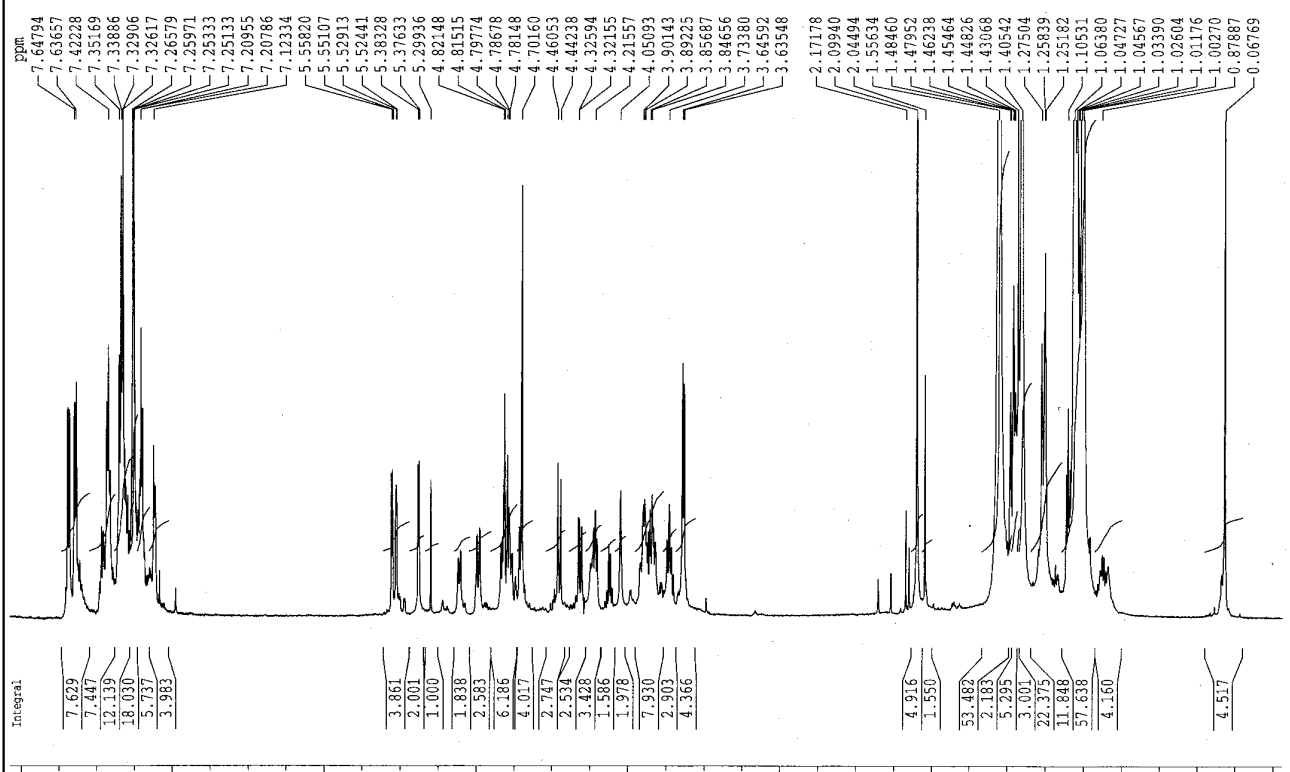


${ }^{13} \mathrm{C}$ NMR of compound 46a $\left(\mathrm{CDCl}_{3}, 151 \mathrm{MHz}\right)$

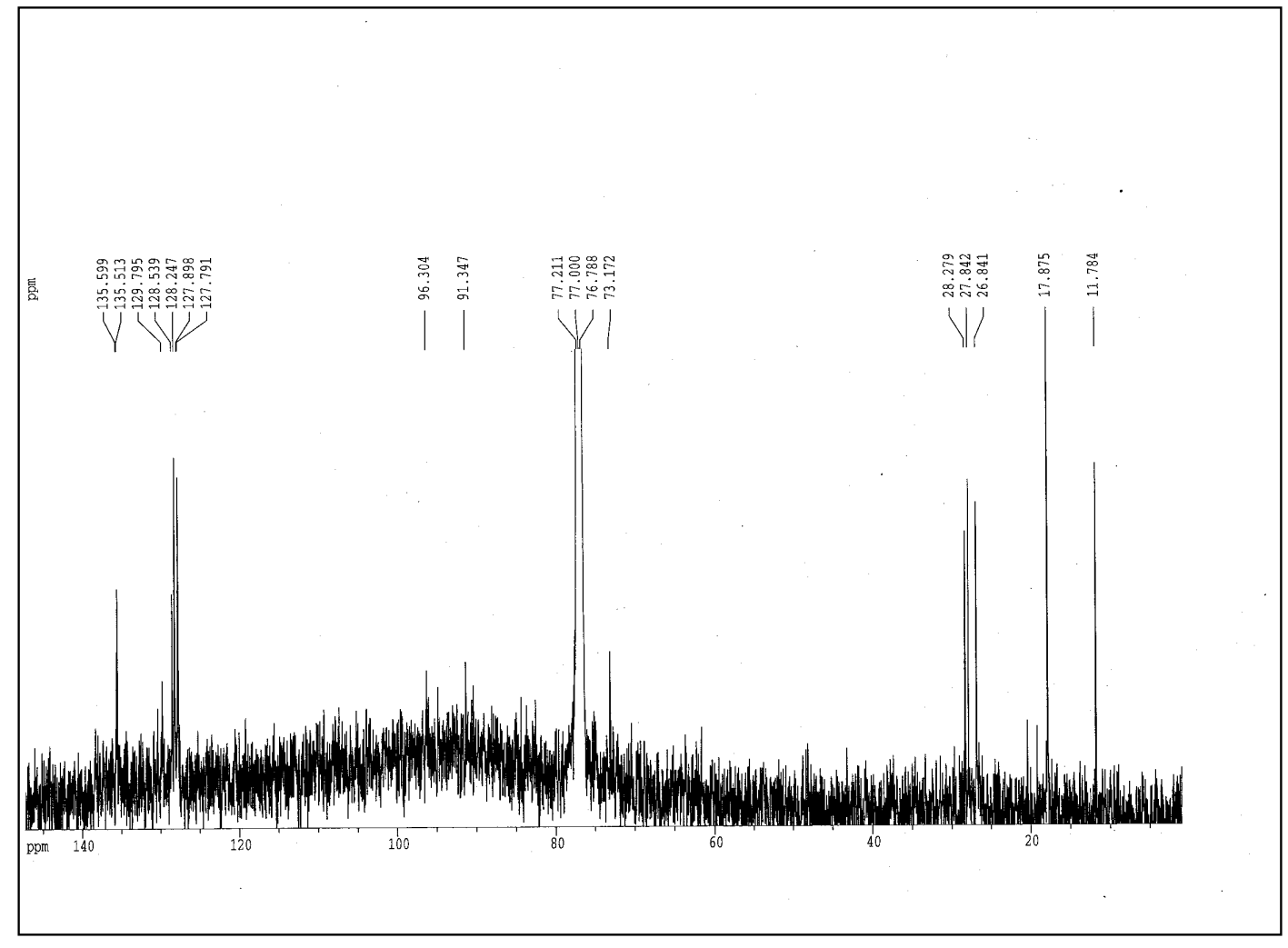

${ }^{1} \mathrm{H}$ NMR of compound 47a $\left(\mathrm{CDCl}_{3}, 600 \mathrm{MHz}\right)$

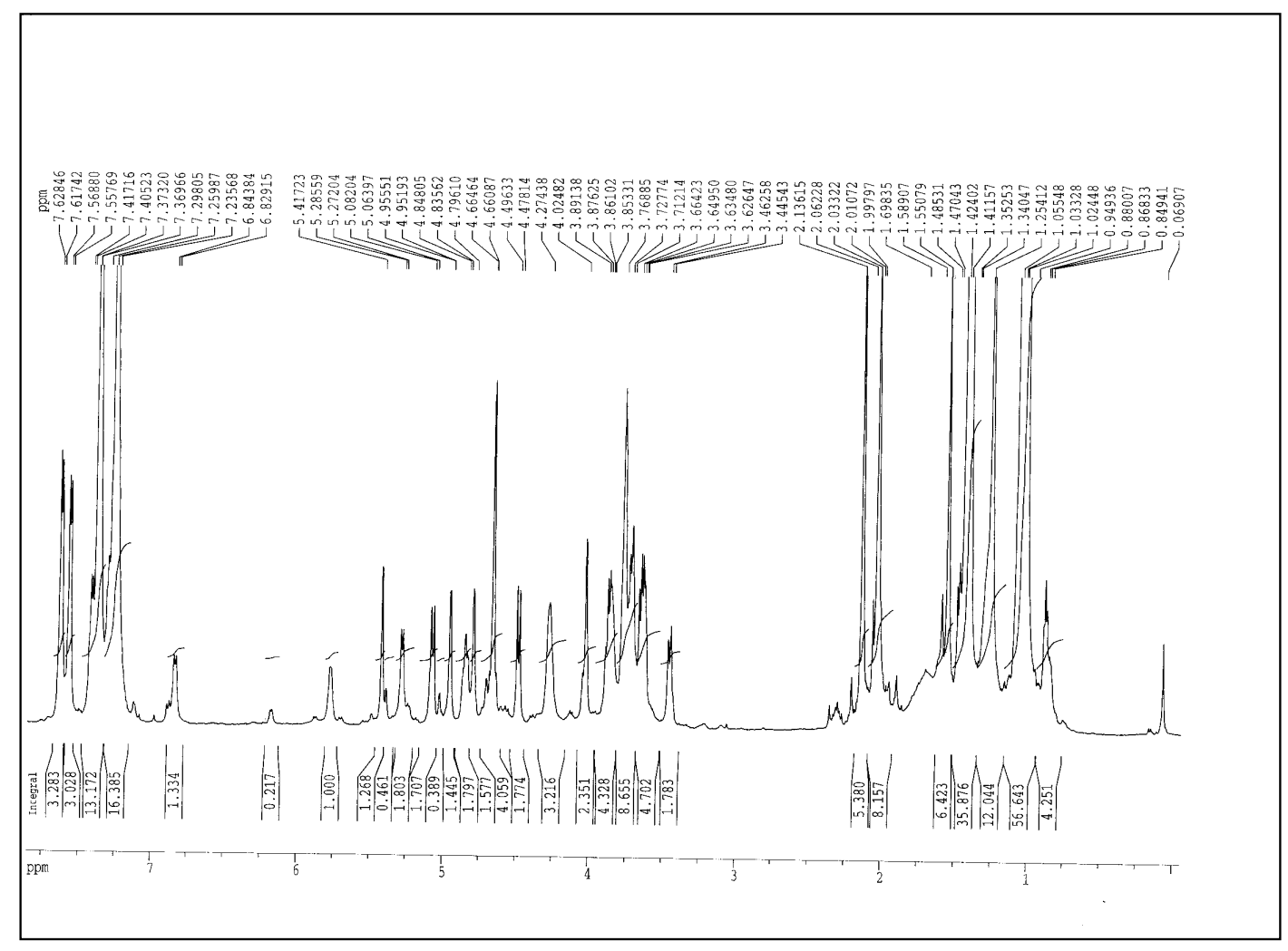


${ }^{13} \mathrm{C}$ NMR of compound $47 \mathbf{a}\left(\mathrm{CDCl}_{3}, 151 \mathrm{MHz}\right)$

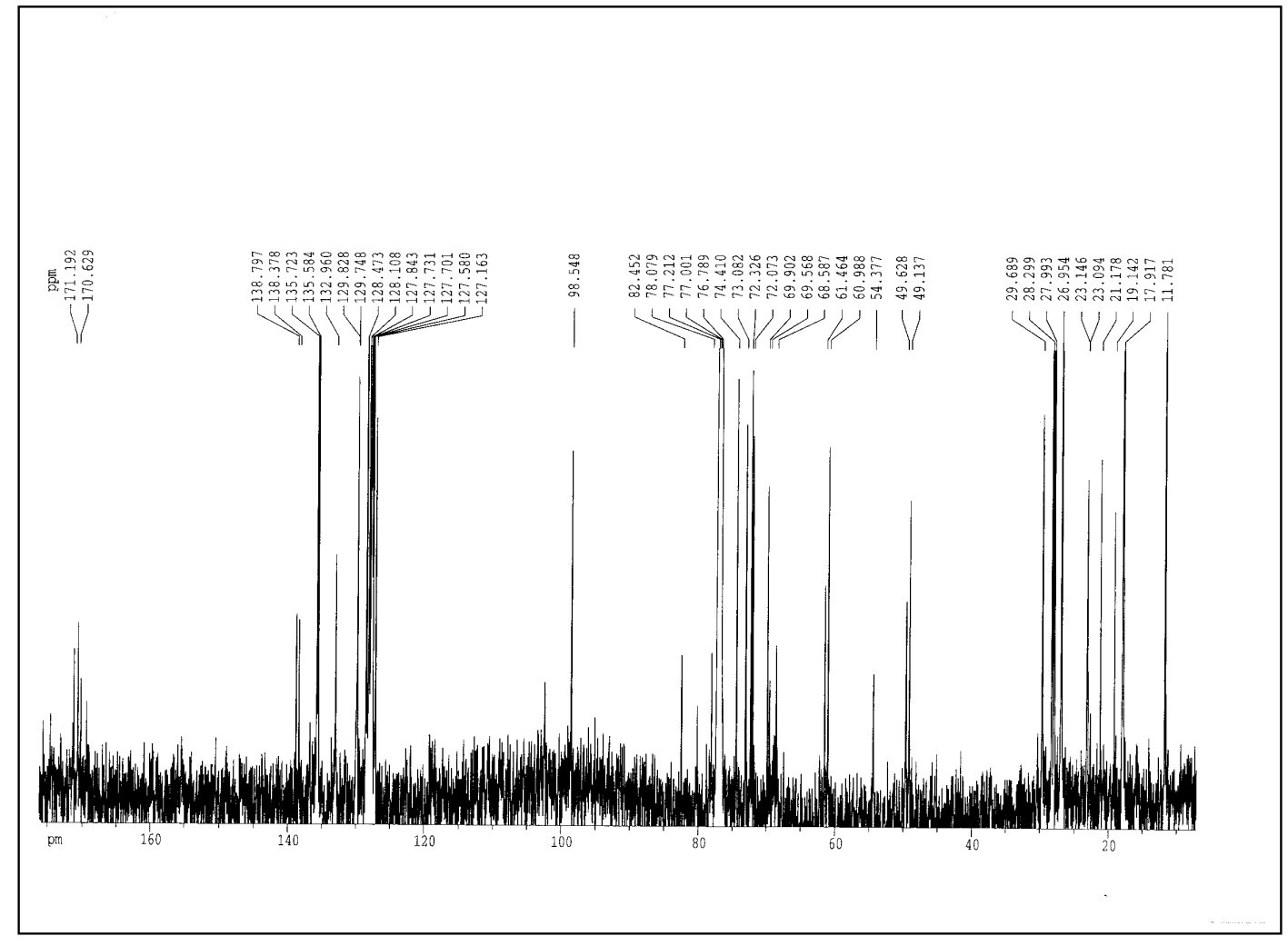




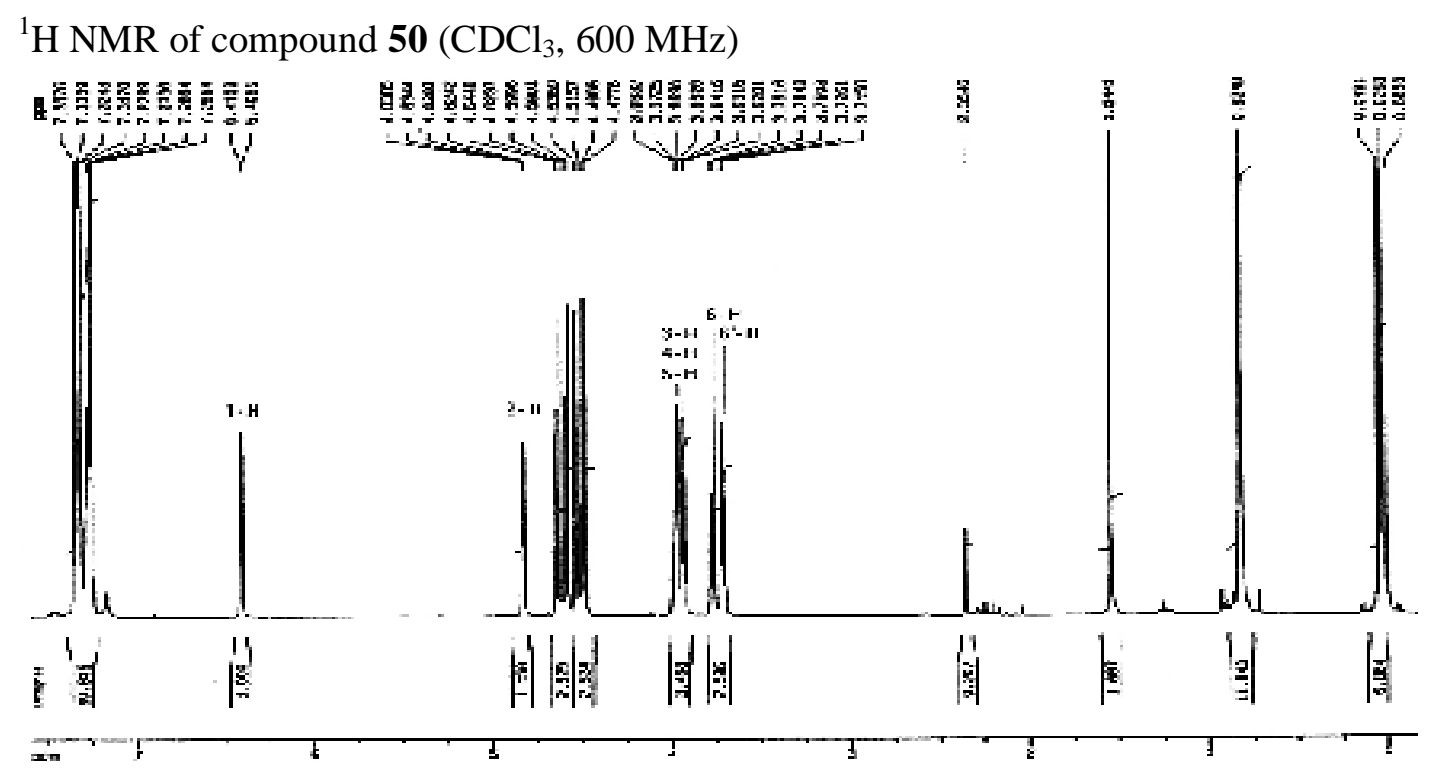

${ }^{13} \mathrm{C}$ NMR of compound $\mathbf{5 0}\left(\mathrm{CDCl}_{3}, 151 \mathrm{MHz}\right)$

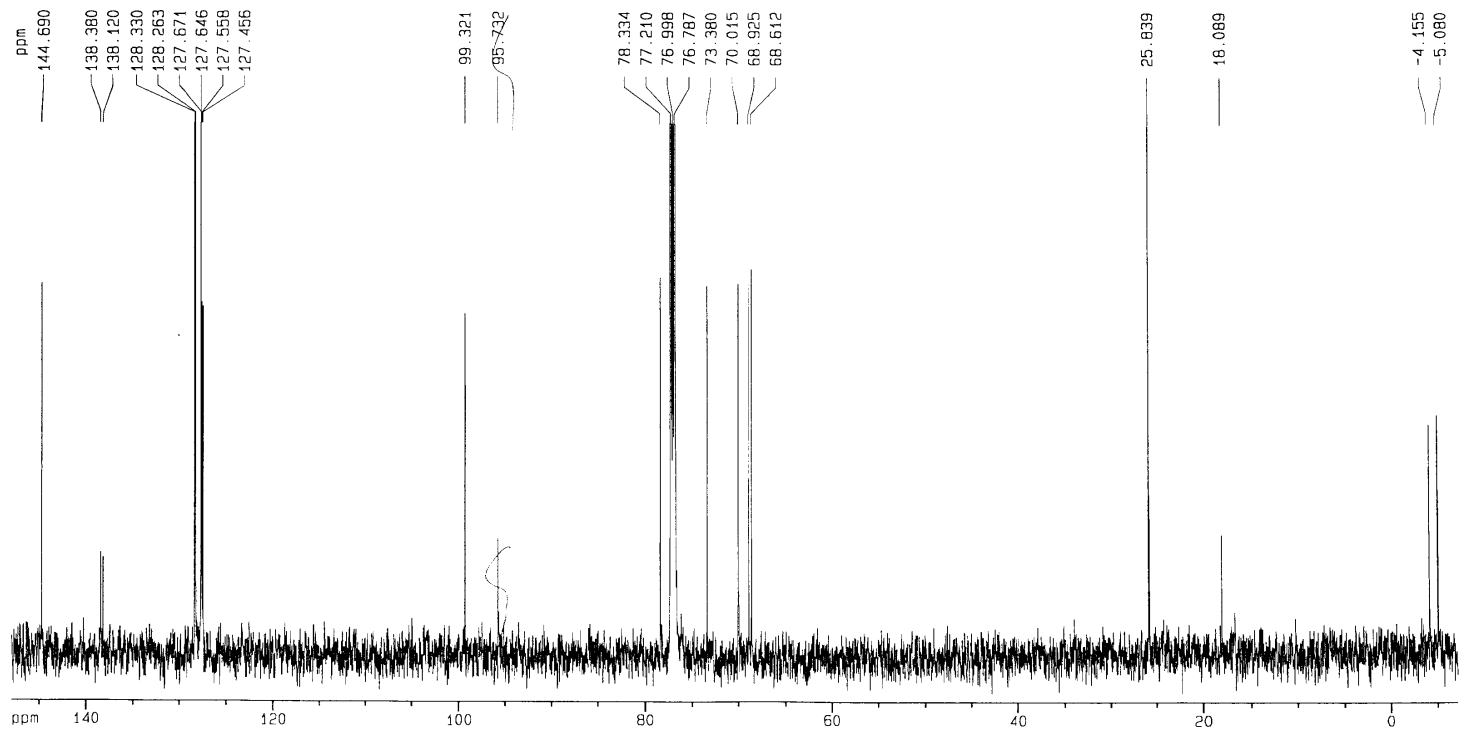




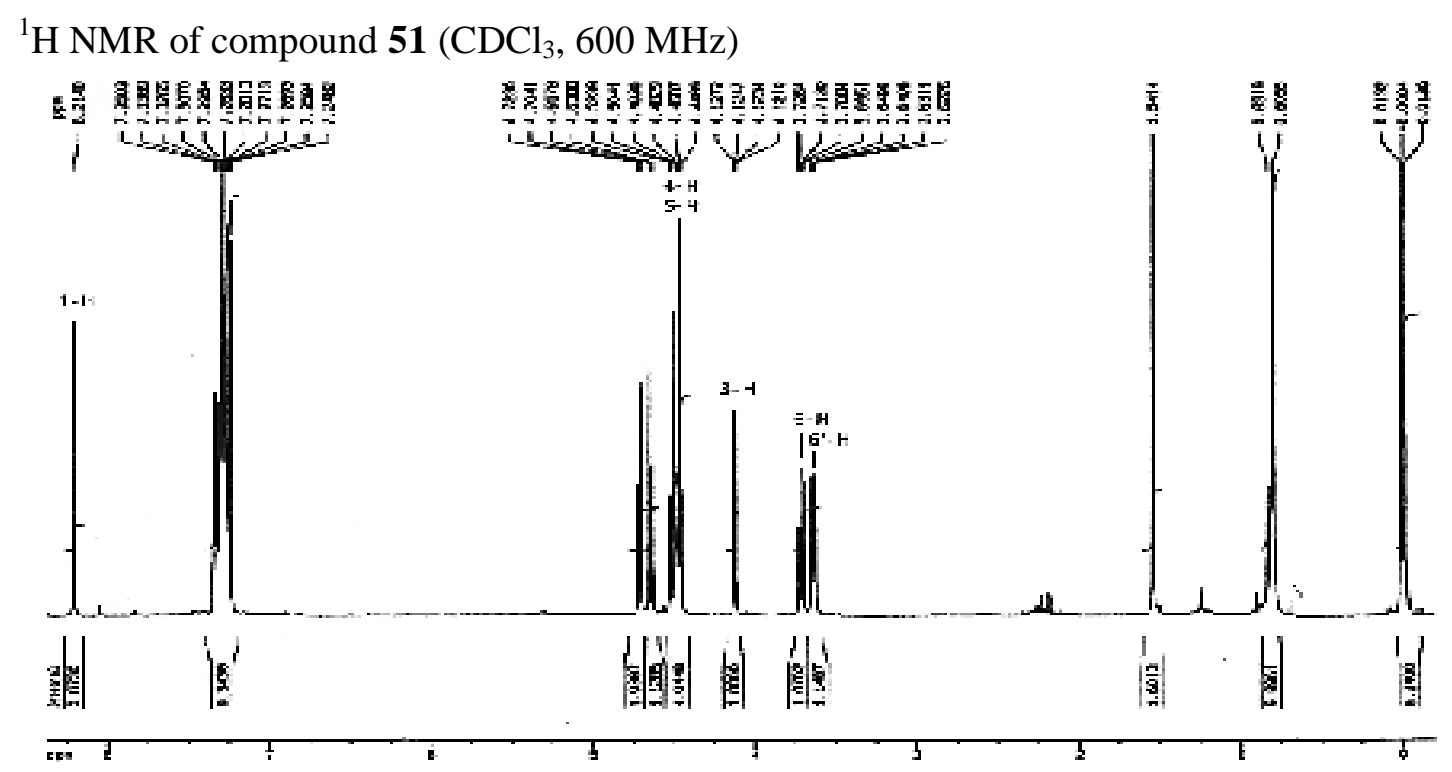

${ }^{13} \mathrm{C}$ NMR of compound $\mathbf{5 1}\left(\mathrm{CDCl}_{3}, 151 \mathrm{MHz}\right)$
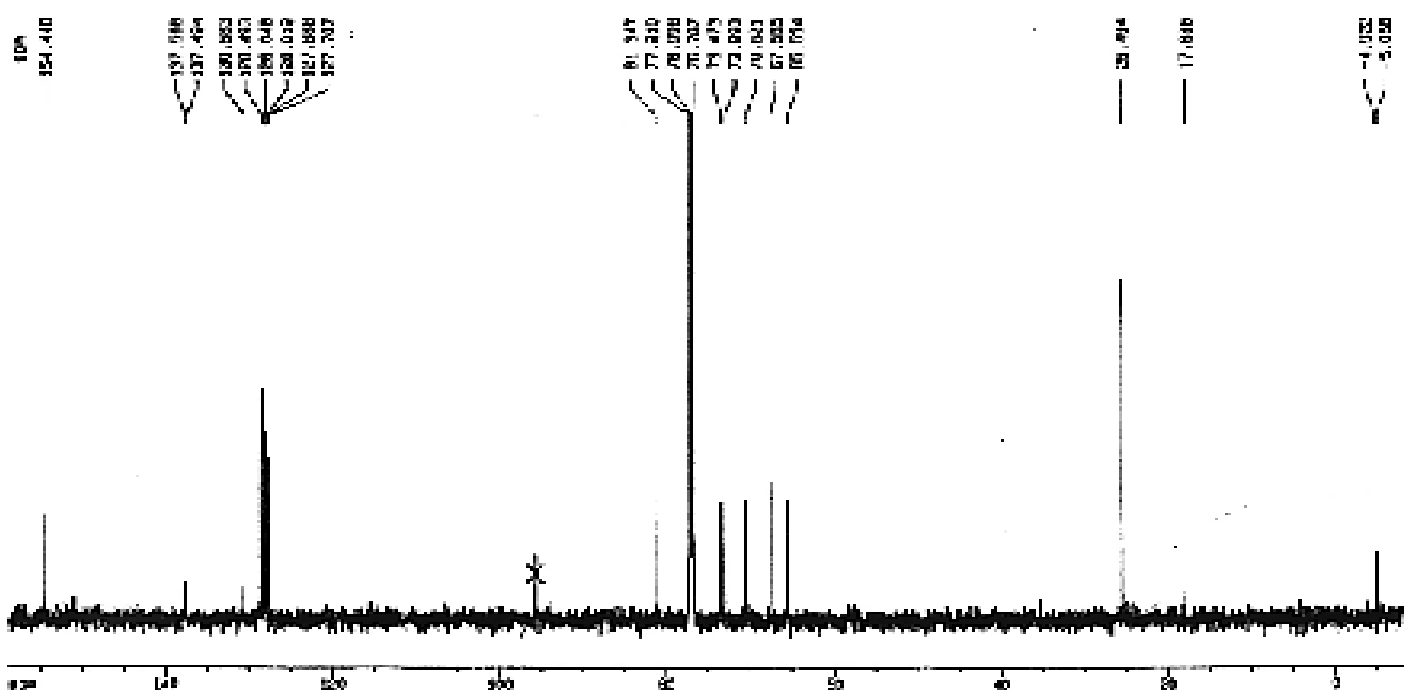


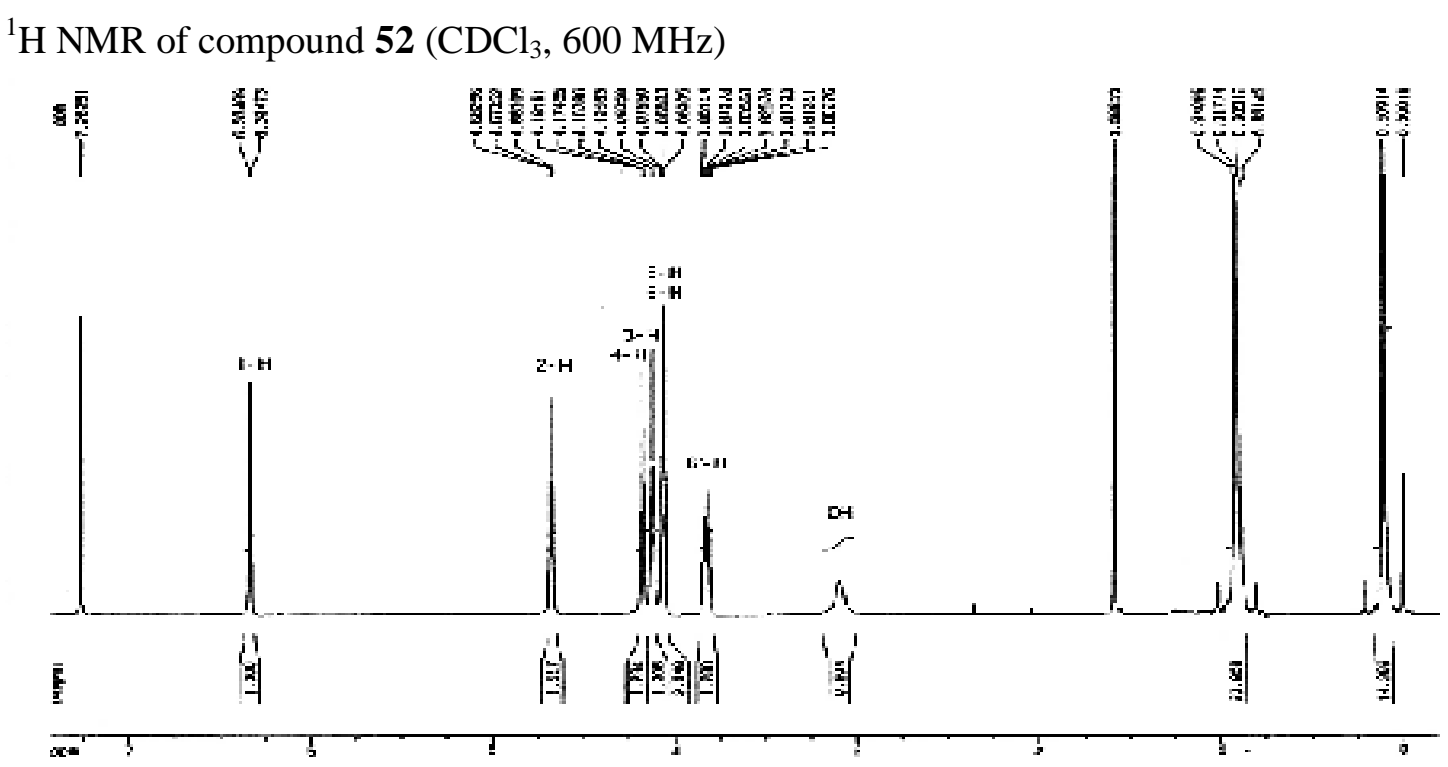

${ }^{13} \mathrm{C}$ NMR of compound $\mathbf{5 2}\left(\mathrm{CDCl}_{3}, 151 \mathrm{MHz}\right)$

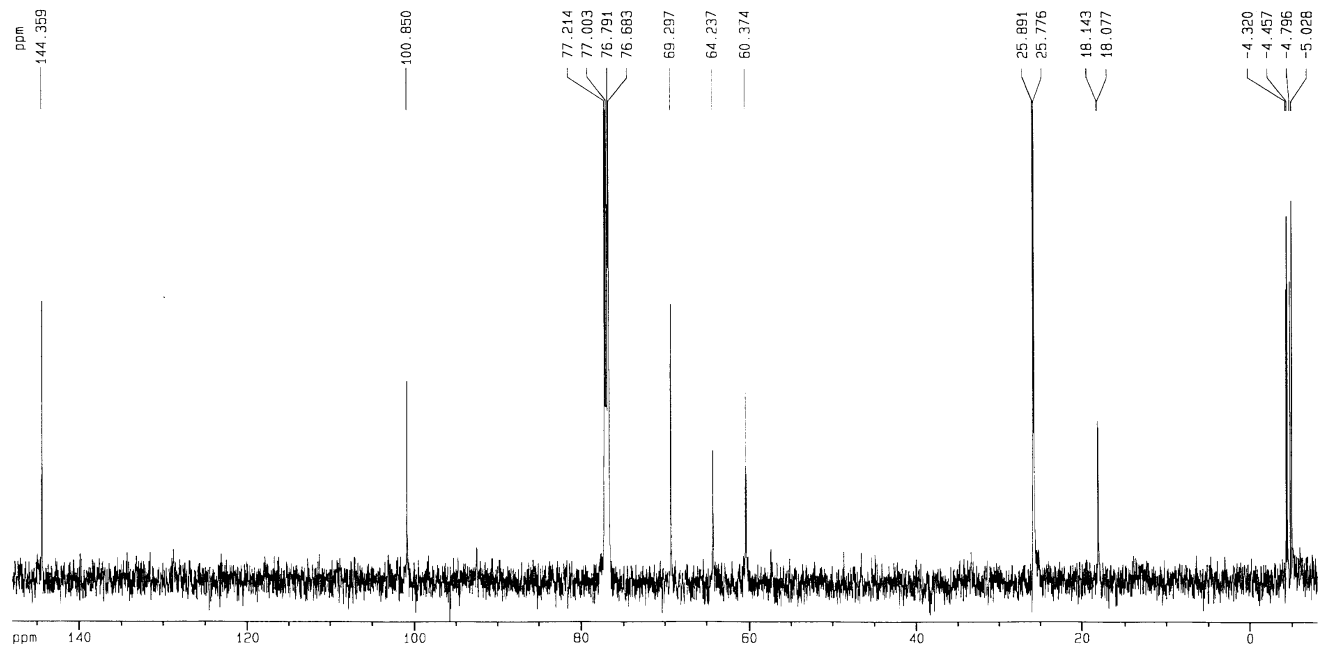




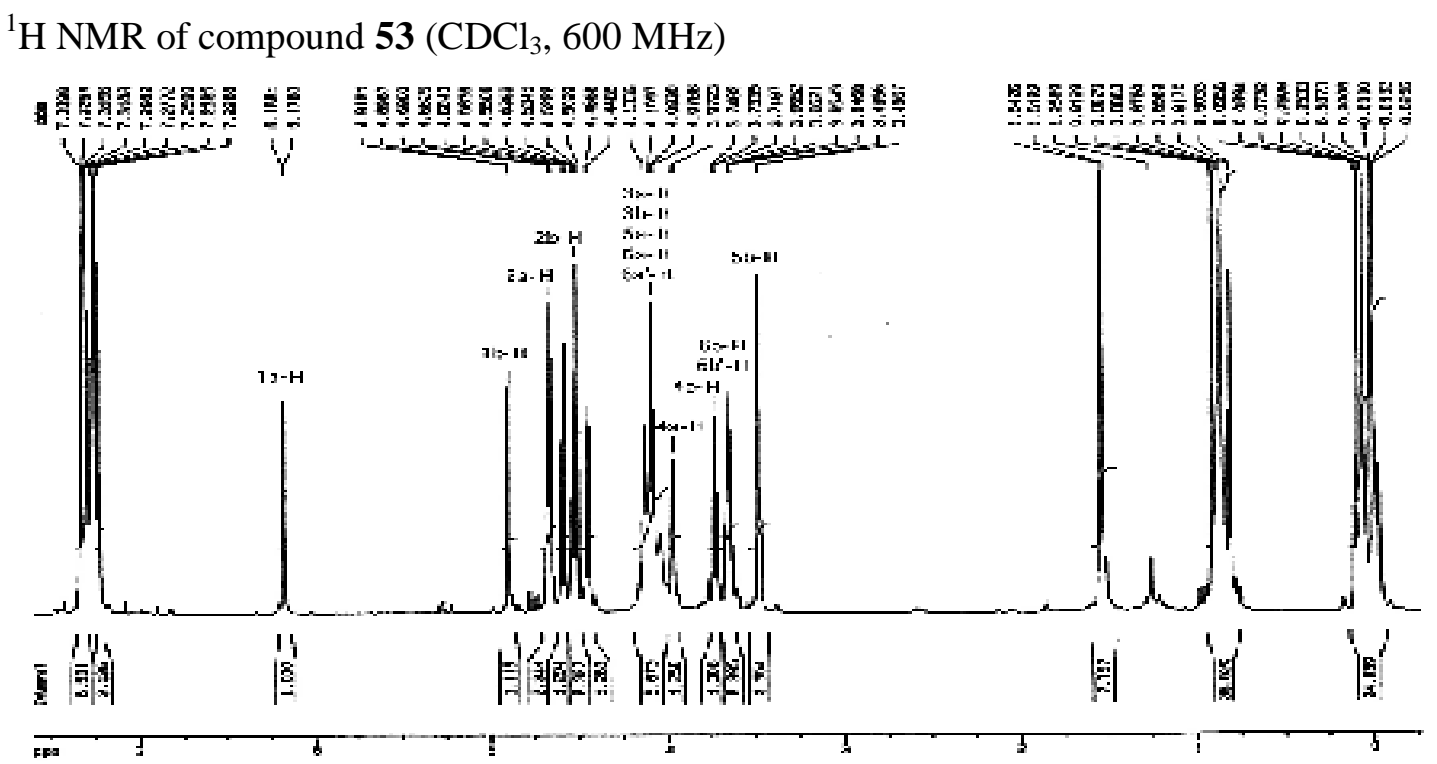

${ }^{13} \mathrm{C}$ NMR of compound $\mathbf{5 3}\left(\mathrm{CDCl}_{3}, 151 \mathrm{MHz}\right)$

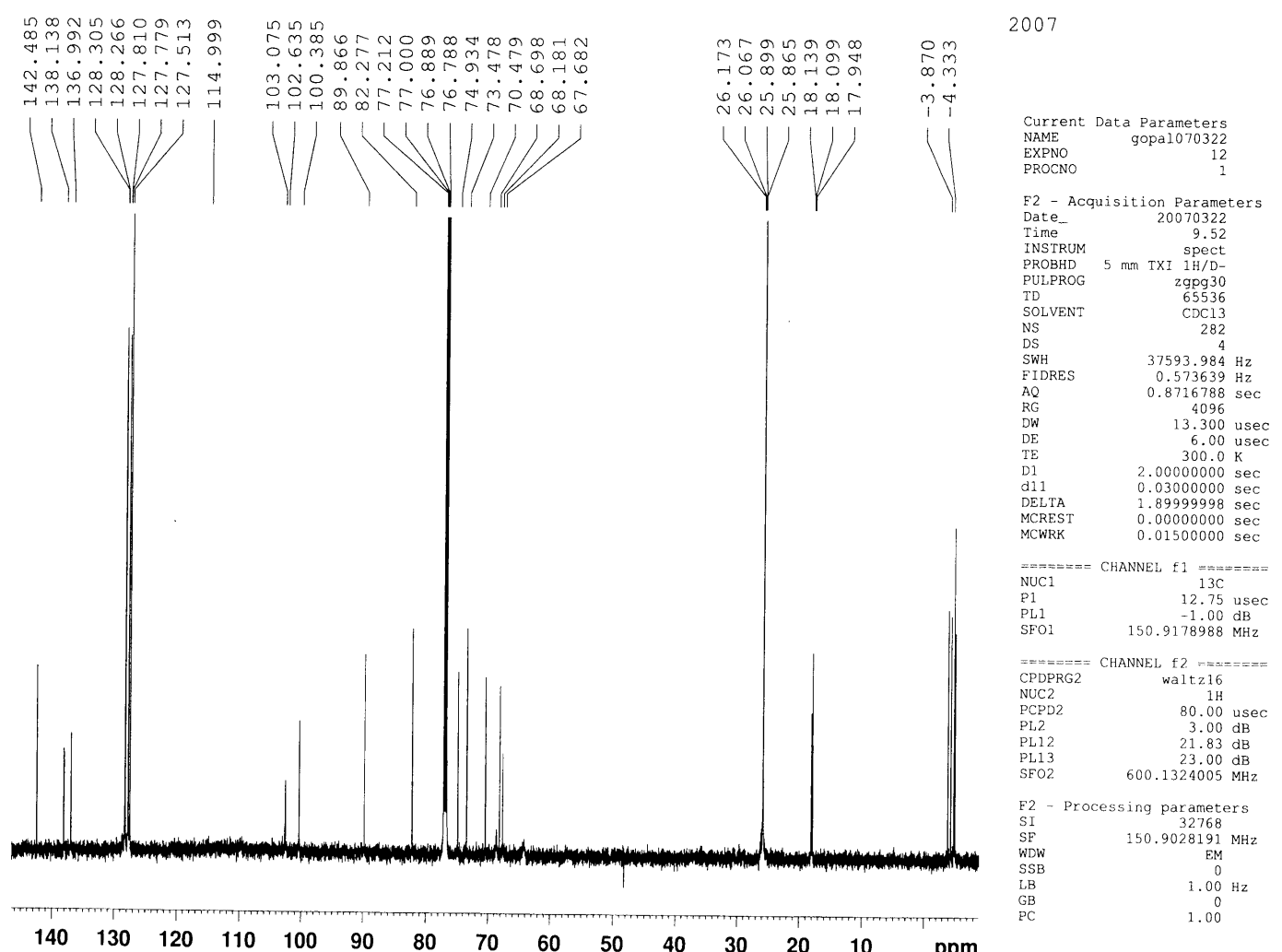



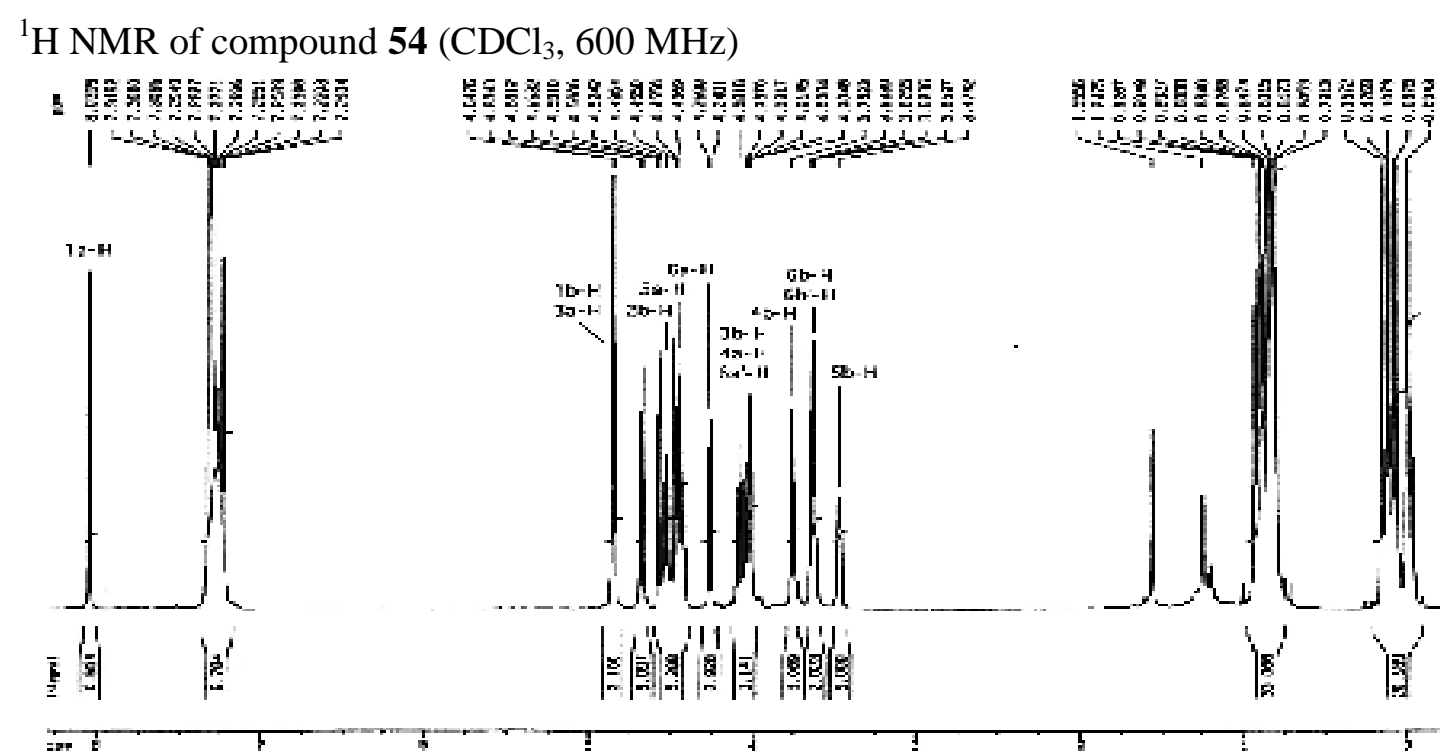

${ }^{13} \mathrm{C}$ NMR of compound $54\left(\mathrm{CDCl}_{3}, 151 \mathrm{MHz}\right)$

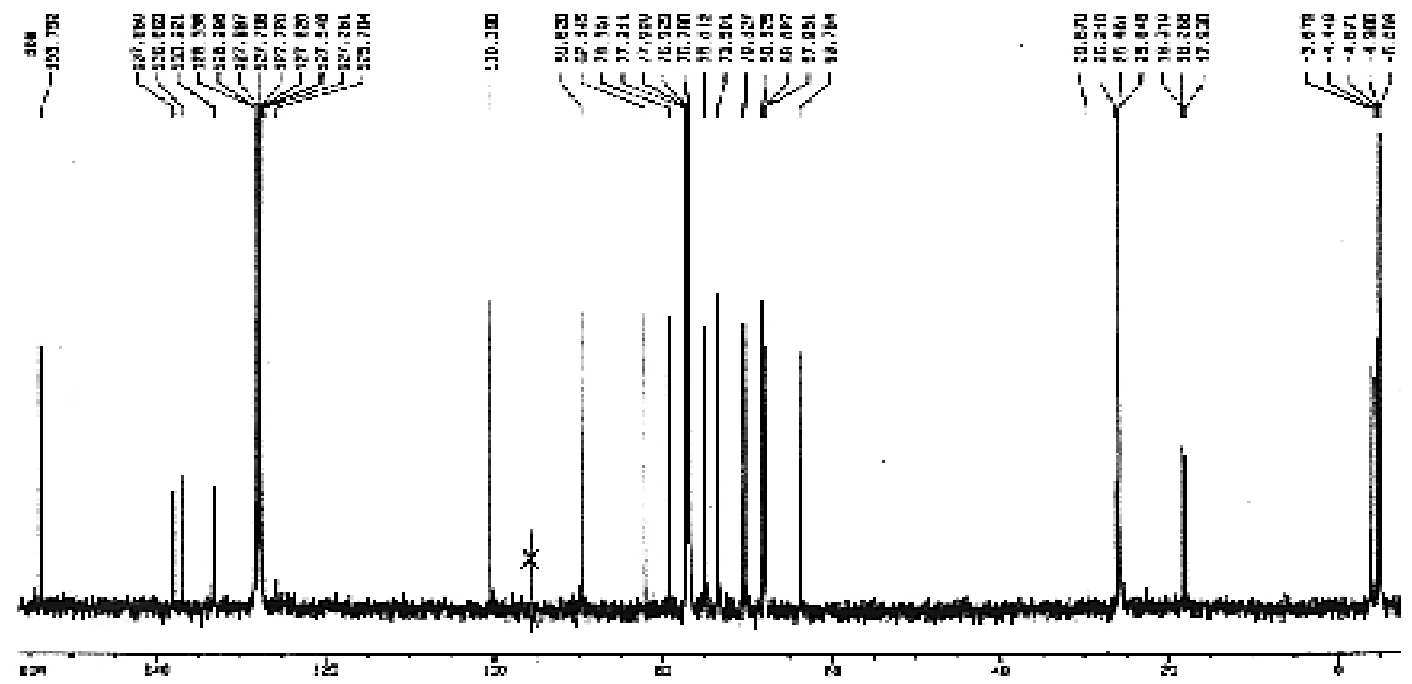




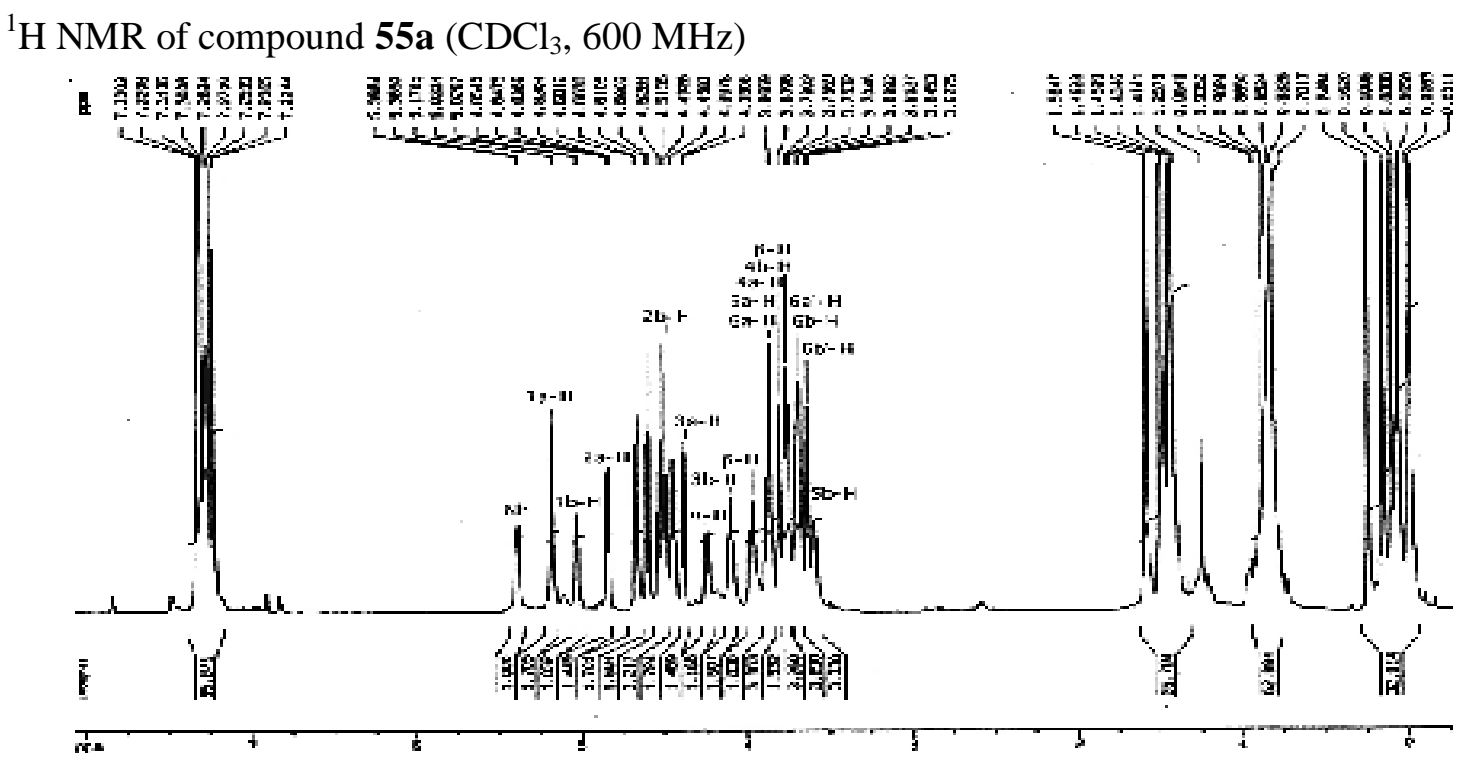

${ }^{13} \mathrm{C}$ NMR of compound $\mathbf{5 5 a}\left(\mathrm{CDCl}_{3}, 151 \mathrm{MHz}\right)$

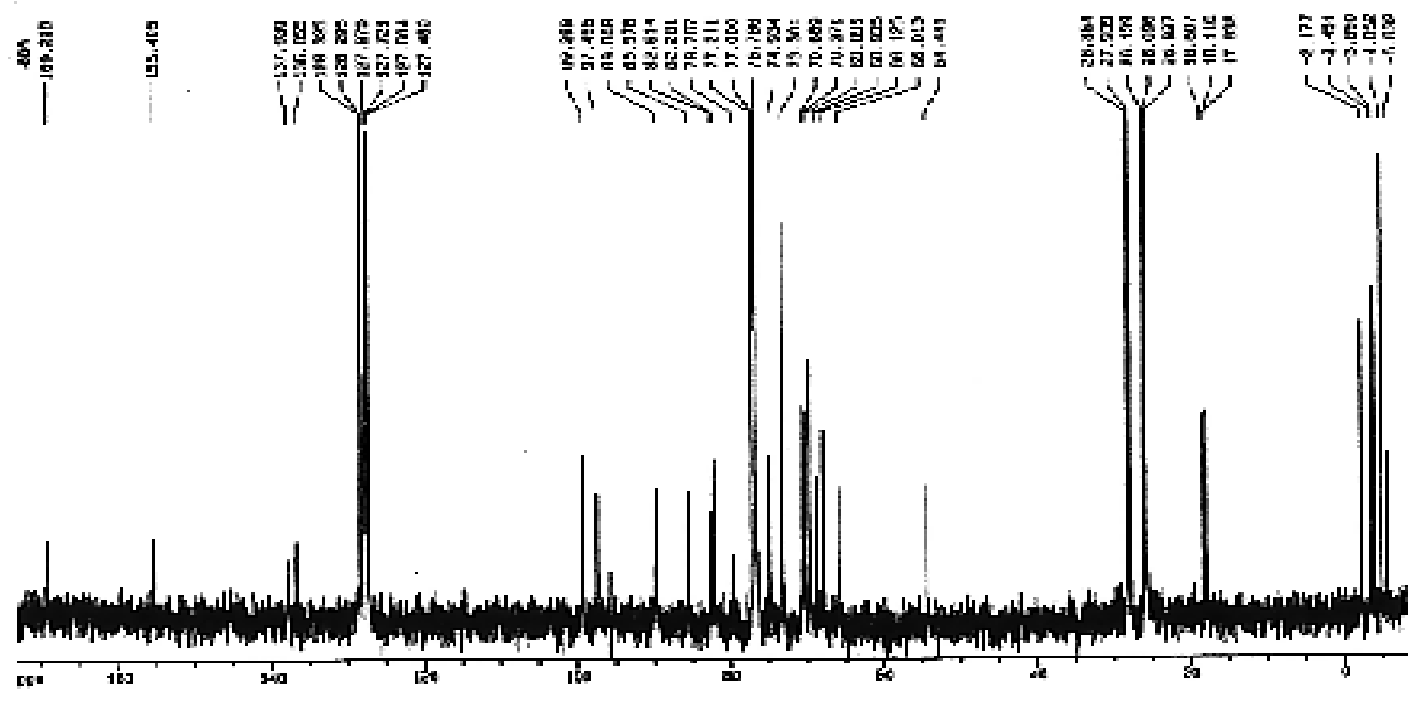

Experimental procedures and physical data for compounds:

General Experimental Methods: Solvents were evaporated under reduced pressure while maintaining the water bath temperature below $40{ }^{\circ} \mathrm{C}$. Chromatography was performed on silica gel for flash chromatography $40 \mu \mathrm{m}$ at 3 bar pressure. For thin-layer chromatography (TLC) plastic sheets of silica gel $60 \mathrm{~F}_{254}$ were used and the compounds visualized by illumination under UV light at $253 \mathrm{~nm}$ and by treatment with a solution of $5 \%\left(\mathrm{NH}_{4}\right)_{2} \mathrm{MoO}_{4}$ and $0.1 \% \mathrm{Ce}\left(\mathrm{SO}_{4}\right)_{2}$ in $10 \% \mathrm{H}_{2} \mathrm{SO}_{4}$ followed by heating to $160{ }^{\circ} \mathrm{C}$. Optical rotations were measured at $25{ }^{\circ} \mathrm{C}$ using the sodium D line. NMR spectra: TMS or the solvent residual peak were used as internal standard. Peak and coupling constant assignments are based on 1D, COSY, HSQC, HMBC and when required on ROESY and NOESY experiments. MALDIMS: 2.5-dihydroxybenzoic acid was used as matrix. FAB-MS: $70 \mathrm{eV}, 70{ }^{\circ} \mathrm{C}$. High resolution 
mass spectra (HRMS) were recorded on an Apex II FT-ICR mass spectrometer (Bruker Daltonik, Bremen, Germany) equipped with a $7 \mathrm{~T}$ actively shielded cryomagnet and an Apollo ESI source. Acquisition was performed with the softwar XMass 6.1.2 (Bruker Daltonik). Solutions of $2 \mathrm{pmol} / \mu \mathrm{L}$ of the carbohydrates in $\mathrm{MeOH}$ were injected using a syringe pump (Harvard Instrumentus, South Natick, USA) with flow rates of $2-3 \mu \mathrm{L} / \mathrm{min}$. If required, solutions of $40 \mathrm{mg} / \mathrm{mL} \mathrm{NaI}$ or KI, respectively, were added to the carbohydrate solutions in a ratio of 1:200 (v/v). Mass spectra were obtained by accumulation of 4-16 scans. Experimental conditions: Capillary Exit Voltage 55-70 V; skimmer $110 \mathrm{~V}$; skimmer $29 \mathrm{~V}$; ionisation pulse length $2500 \mu \mathrm{s}$. Calibration was performed externally with fragment ions produced by CID of Angiotensin I (Bachem, Weil am Rhein, Germany). Where its recovery is stated, yields are calculated based on reagents consumed.

$16 \alpha, 16 \beta$

$O$-(2,3,4,6-Tetra- $O$-benzyl- $\alpha$-D-galactopyranosyl)-( $1 \rightarrow 3)-6-O$-(triisopropylsilyl)-Dgalactal $(16 \alpha)$ and $O$-(2,3,4,6-Tetra- $O$-benzyl- $\beta$-D-galactopyranosyl)-(1 $\rightarrow 3)-6-O$ (triisopropylsilyl)- $\beta$-D-galactal (16 $)$. A mixture of $2(12.0 \mathrm{~g}, 4.2 \mathrm{mmol})$ and $\mathbf{1 5}^{[17]}(4.3 \mathrm{~g}$, $6.3 \mathrm{mmol})$ was dissolved in $\mathrm{CH}_{2} \mathrm{Cl}_{2} / \mathrm{Et}_{2} \mathrm{O}(1: 2,30 \mathrm{~mL})$, cooled to $0^{\circ} \mathrm{C}$ and added dropwise a mixture of zinc(II) triflate $(55 \mathrm{mg}, 0.26 \mathrm{mmol})$ in $\mathrm{MeCN}(3 \mathrm{~mL})$. After $30 \mathrm{~min}$ triethylamine was added and the mixture was washed with water. The solution was dried with $\mathrm{MgSO}_{4}$ and the solvent removed under reduced pressure. Purification by flash chromatography (toluene/ethyl acetate $=9: 1)$ afforded $\alpha$-diastereomer $16 \alpha(1.62 \mathrm{~g}, 2.0 \mathrm{mmol}, 47 \%)$ and $\beta$ diastereomer $16 \beta(180 \mathrm{mg}, 0.22 \mathrm{mmol}, 5 \%)$ as slightly yellow oil. $16 \alpha: R_{\mathrm{f}}=0.85$ (petroleum ether/ethyl acetate $=3: 1) ;[\alpha]_{\mathrm{D}}=+34.2\left(\mathrm{c}=1, \mathrm{CHCl}_{3}\right) .{ }^{1} \mathrm{H} \mathrm{NMR}\left(600 \mathrm{MHz}, \mathrm{CDCl}_{3}\right): \delta=$ 7.31-7.26 (m, $20 \mathrm{H}$, arom. H), $6.40\left(\mathrm{dd}, J_{1,2}=6.2 \mathrm{~Hz}, J_{1,3}=1.0 \mathrm{~Hz}, 1 \mathrm{H}, 1 \mathrm{a}-\mathrm{H}\right), 4.95\left(\mathrm{~d}, J_{1,2}=\right.$ $3.8 \mathrm{~Hz}, 1 \mathrm{H}, 1 \mathrm{~b}-\mathrm{H}), 4.93(\mathrm{~d}, J=14.4 \mathrm{~Hz}, 1 \mathrm{H}$, benzyl. H), 4.82 (d, $J=11.7 \mathrm{~Hz}, 2 \mathrm{H}$, benzyl. $\mathrm{H})$, 4.72-4.70 (m, $2 \mathrm{H}$, benzyl. H), 4.64-4.62 (m, $1 \mathrm{H}, 2 \mathrm{a}-\mathrm{H}), 4.58(\mathrm{~d}, J=12.1 \mathrm{~Hz}, 2 \mathrm{H}$, benzyl. H), 4.56 (d, $J=4.4 \mathrm{~Hz}, 1 \mathrm{H}, 3 \mathrm{a}-\mathrm{H}), 4.45$ (d, $J=9.9 \mathrm{~Hz}, 2 \mathrm{H}$, benzyl. H), 4.13-4.07 (m, $2 \mathrm{H}, 4 \mathrm{a}-\mathrm{H}, 6 \mathrm{a}-\mathrm{H}), 4.05-3.96$ (m, 4 H, 3b-H, 5b-H, 6a-H, 6b-H), 3.95-3.90 (m, 2 H, 6b'-H, 6a'$\mathrm{H}), 3.53(\mathrm{~d}, J=7.7 \mathrm{~Hz}, 2 \mathrm{H}, 4 \mathrm{~b}-\mathrm{H}, 5 \mathrm{a}-\mathrm{H}), 1.12-1.05$ (m, $21 \mathrm{H}, \mathrm{TIPS}) .{ }^{13} \mathrm{C}$ NMR (151 MHz, $\left.\mathrm{CDCl}_{3}\right): \delta=145.3(\mathrm{C}-1 \mathrm{a}), 138.5-127.3$ (arom. C), 100.2 (C-2a), 96.7 (C-1b), 79.5 (C-3b), 77.5, 75.4 (C-2b), 74.8 (C-6b), 74.1, 73.5, 72.8, 70.1 (C-3a), 70.0 (C-4a), 68.8 (C-5a), 68.6 (C-4b), 67.0, 61.5 (C-5b), 61.7 (C-6a), 18.0, 11.9. MALDI-MS (positive mode, DHB): [M + $\mathrm{Na}$ ]+: $m / z$ calcd.: 847.3, found: 846.5. $\mathrm{C}_{49} \mathrm{H}_{64} \mathrm{O}_{9} \mathrm{Si}(825.11) . \mathrm{C}_{49} \mathrm{H}_{64} \mathrm{O}_{9} \mathrm{Si} \bullet 0.5 \mathrm{H}_{2} \mathrm{O}$ (834.1), calcd.: C: 70.51, H: 7.85, found: C: 70.39, H: 7.56.

16 $\beta$ : $\mathrm{R}_{\mathrm{f}}=0.80$ (petroleum ether/ethyl acetate $\left.=3: 1\right) ;[\alpha]_{\mathrm{D}}=+18.3\left(\mathrm{c}=1, \mathrm{CHCl}_{3}\right) .{ }^{1} \mathrm{H} \mathrm{NMR}$ $\left(250 \mathrm{MHz}, \mathrm{CDCl}_{3}\right): \delta=4.55\left(\mathrm{~d}, J_{1,2}=7.8 \mathrm{~Hz}, 1 \mathrm{H}, 1 \mathrm{~b}-\mathrm{H}\right)$.

17

$O$-(2,3,4,6-Tetra- $O$-benzyl- $\alpha$-D-galactopyranosyl)-( $1 \rightarrow 3)$-4- $O$-benzyl-6- $O$ -

(triisopropylsilyl)-D-galactal (17). To a mixture of $\mathbf{1 6} \alpha(1.8 \mathrm{~g}, 2.2 \mathrm{mmol})$ and DMF (20 mL) was added $\mathrm{NaH}$ ( $230 \mathrm{mg}, 6.6 \mathrm{mmol})$, stirred for $15 \mathrm{~min}$ and then added dropwise $\mathrm{BnBr}(0.79$ $\mathrm{mL}, 6.6 \mathrm{mmol})$. Stirring was then continued for $15 \mathrm{~h}$ at $40{ }^{\circ} \mathrm{C}$. After termination of the reaction ethyl acetate $(20 \mathrm{~mL})$ was added and the organic phase washed with water $(3 \times 5$ $\mathrm{mL}$ ). The organic phase was dried with $\mathrm{MgSO}_{4}$ concentrated under reduced pressure. Purification by flash chromatography (petroleum ether/ethyl acetate $=10: 1)$ afforded $17(1.2$ g, $1.3 \mathrm{mmol}, 60 \%$ ) as slightly yellow oil. $\mathrm{R}_{\mathrm{f}}=0.70$ (toluene/ethyl acetate $=5: 1$ ); $[\alpha]_{\mathrm{D}}=+17.9$ $\left(\mathrm{c}=1, \mathrm{CHCl}_{3}\right) .{ }^{1} \mathrm{H}$ NMR $\left(600 \mathrm{MHz}, \mathrm{CDCl}_{3}\right): \delta=7.38-7.23\left(\mathrm{~m}, 25 \mathrm{H}\right.$, arom. H) $6.26\left(\mathrm{dd}, J_{1,2}\right.$ $\left.=6.2 \mathrm{~Hz}, J_{1,3}=1.0 \mathrm{~Hz}, 1 \mathrm{H}, 1 \mathrm{a}-\mathrm{H}\right), 5.13(\mathrm{br} \mathrm{s}, 1 \mathrm{H}, 1 \mathrm{~b}-\mathrm{H}), 4.96(\mathrm{~d}, J=11.4 \mathrm{~Hz}, 2 \mathrm{H}$, benzyl. H), 4.90-4.88 (m, $1 \mathrm{H}, 2 \mathrm{a}-\mathrm{H}), 4.75(\mathrm{~d}, J=11.5 \mathrm{~Hz}, 2 \mathrm{H}$, benzyl. H), 4.68-4.61 (m, $3 \mathrm{H}$, benzyl. H), 4.58 (d, $J=11.4 \mathrm{~Hz}, 1 \mathrm{H}$, benzyl. H), 4.51 (d, $J=11.4 \mathrm{~Hz}, 1 \mathrm{H}$, benzyl. H), 4.42 
$(\mathrm{d}, J=11.4 \mathrm{~Hz}, 1 \mathrm{H}$, benzyl. H), 4.33 (br s, $1 \mathrm{H}, 3 \mathrm{a}-\mathrm{H}), 4.13\left(\mathrm{dd}, J_{5,4}=J_{5,6}=6.0 \mathrm{~Hz}, 1 \mathrm{H}, 5 \mathrm{~b}-\right.$ H), 4.06-4.04 (m, 1 H, 4b-H), 4.05 (dd, $\left.J_{2,1}=3.6 \mathrm{~Hz}, J_{2,3}=10.5 \mathrm{~Hz}, 1 \mathrm{H}, 2 \mathrm{~b}-\mathrm{H}\right), 3.95$ (br s, 1 $\mathrm{H}, 6 \mathrm{a}-\mathrm{H}), 3.93$ (br s, $1 \mathrm{H}, 4 \mathrm{a}-\mathrm{H}), 3.90\left(\mathrm{dd}, J_{3,4}=3.0 \mathrm{~Hz}, J_{3,2}=10.5 \mathrm{~Hz}, 1 \mathrm{H}, 3 \mathrm{~b}-\mathrm{H}\right), 3.89-3.87$ $\left(\mathrm{m}, 1 \mathrm{H}, 6 \mathrm{a}^{\prime}-\mathrm{H}\right), 3.70(\mathrm{~s}, 1 \mathrm{H}, 5 \mathrm{a}-\mathrm{H}), 3.56\left(\mathrm{dd}, J_{6,6^{\prime}}=9.6 \mathrm{~Hz}, J_{6,5}=6.0 \mathrm{~Hz}, 1 \mathrm{H}, 6 \mathrm{~b}-\mathrm{H}\right), 3.51$ $\left(\mathrm{dd}, J_{6,6}=9.6 \mathrm{~Hz}, J_{6,5}=6.0 \mathrm{~Hz}, 1 \mathrm{H}, 6 \mathrm{~b}^{\prime}-\mathrm{H}\right), 1.12-1.05$ (m, $21 \mathrm{H}$, TIPS). ${ }^{13} \mathrm{C}$ NMR $(151 \mathrm{MHz}$, $\mathrm{CDCl}_{3}$ ): $\delta=144.1$ (C-1a), 138.9-127.4 (30 C, arom. C), 101.4 (C-2a), 99.3 (C-1b), 78.9 (C3b), 77.7 (C-4b), 76.9 (C-2b), 76.8, 75.6 (C-4a), 74.8, 73.4, 72.9, 69.9 (C-5b), 69.4 (C-6b), 67.1 (C-5a), 61.5 (C-6a), 18.8 (6 C), 12.5 (6 C). MALDI-MS (positive mode, DHB): [M + $\mathrm{Na}]^{+}: m / z$ calcd.: 937.4, found: 936.4. $\mathrm{C}_{56} \mathrm{H}_{70} \mathrm{O}_{9} \mathrm{Si}(915.24)$ calcd.: C: 73.49, H: 7.71, found: C: $72.85, \mathrm{H}: 7.95$.

18

$O$-(2,3,4,6-Tetra- $O$-benzyl- $\alpha$-D-galactopyranosyl)-( $1 \rightarrow 3)$-di-4,6- $O$-benzyl-D-galactal $(18)$. To a solution of $16(1.8 \mathrm{~g}, 2.2 \mathrm{mmol})$ in THF $(15 \mathrm{~mL})$ was added TBAF in THF $(4.7 \mathrm{~mL}, 1 \mathrm{M})$ and the mixture stirred for $30 \mathrm{~min}$ at $\mathrm{rt}$. The solution was concentrated under reduced pressure, diluted in dry DMF, and then $\mathrm{NaH}(170 \mathrm{mg}, 6.6 \mathrm{mmol})$ and $\mathrm{BnBr}(0.8 \mathrm{~mL}, 6.6$ $\mathrm{mmol})$ added dropwise at $\mathrm{rt}$. After $18 \mathrm{~h}$ of stirring at $\mathrm{rt}$ the reaction was terminated by adding methanol; then ethyl acetate $(50 \mathrm{~mL})$ added and the mixture washed with water $(3 \times 30 \mathrm{~mL})$. The organic phase was separated and the residue purified by flash chromatography (toluene/ethyl acetate $=20: 1)$ which afforded $18(1.2 \mathrm{~g}, 1.3 \mathrm{mmol}, 61 \%)$ as colourless oil. $\mathrm{R}_{\mathrm{f}}$ $=0.70$ (toluene/ethyl acetate $=5: 1) ;[\alpha]_{\mathrm{D}}=+45.7\left(\mathrm{c}=1, \mathrm{CHCl}_{3}\right) .{ }^{1} \mathrm{H}$ NMR $(250 \mathrm{MHz}$, $\left.\mathrm{CDCl}_{3}\right): \delta=7.40-7.19\left(\mathrm{~m}, 30 \mathrm{H}\right.$, arom. H), $6.28\left(\mathrm{dd}, J_{1,2}=6.2 \mathrm{~Hz}, J_{1,3}=1.1 \mathrm{~Hz}, 1 \mathrm{H}, 1 \mathrm{a}-\mathrm{H}\right)$, $5.18\left(\mathrm{~d}, J_{1,2}=3.5 \mathrm{~Hz}, 1 \mathrm{H}, 1 \mathrm{~b}-\mathrm{H}\right), 4.98(\mathrm{~d}, J=11.4 \mathrm{~Hz}, 1 \mathrm{H}$, benzyl. H), 4.92-4.8 (m, $3 \mathrm{H}, 2 \mathrm{a}-$ $\mathrm{H}$, benzyl H), 4.75-4.30 (m, $11 \mathrm{H}$, benzyl H), 4.20 (br s, $1 \mathrm{H}, 3 \mathrm{a}-\mathrm{H}), 4.12-4.05$ (m, $3 \mathrm{H}, 2 \mathrm{~b}-\mathrm{H}$, 4b-H, 5b-H), 4.06-4.04 (m, $1 \mathrm{H}, 4 \mathrm{~b}-\mathrm{H}), 4.05$ (dd, $\left.J_{2,1}=3.6 \mathrm{~Hz}, J_{2,3}=10.5 \mathrm{~Hz}, 2 \mathrm{~b}-\mathrm{H}\right), 3.95$ (br s, $1 \mathrm{H}, 6 \mathrm{a}-\mathrm{H}), 3.93$ (s, $2 \mathrm{H}, 4 \mathrm{a}-\mathrm{H}, 6 \mathrm{a}-\mathrm{H}), 3.88\left(\mathrm{dd}, J_{6,6}=J_{6,5}=8.4 \mathrm{~Hz}, 6 \mathrm{a}^{\prime}-\mathrm{H}\right), 3.62-3.50$ (m, 3 $\mathrm{H}, 5 \mathrm{a}-\mathrm{H}, 6 \mathrm{a}-\mathrm{H}, 6 \mathrm{a}-\mathrm{H}) .{ }^{13} \mathrm{C} \mathrm{NMR}\left(63 \mathrm{MHz}, \mathrm{CDCl}_{3}\right): \delta=144.0$ (C-1a), 138.7-127.4 (36 C, arom. C), 101.3 (C-2a), 99.1 (C-1b), 78.6 (C-3b), 77.5 (C-4b), 76.5 (C-2b), 75.7, 75.4 (C-4a), 74.7, 73.4, 73.2, 72.8, 69.9 (C-5b), 69.4 (C-6b), 68.5 (C-6a). MALDI-MS (positive mode, DHB): $[\mathrm{M}+\mathrm{Na}]+: \mathrm{m} / z$ calcd.: 871.4 , found: 871.2. $\mathrm{C}_{54} \mathrm{H}_{56} \mathrm{O}_{9}(849.02)$, calcd.: C: $76.39, \mathrm{H}$ : 6.65, found: C: 76.22, H: 7.10.

19

$O$-(2,3,4,6-Tetra- $O$-benzyl- $\alpha$-D-galactopyranosyl)-( $1 \rightarrow 3)-4-O$-benzyl-2-nitro-6- $O$ -

(triisopropylsilyl)-D-galactal (19). Following general procedure A from 17 (211 mg, 0.23 $\mathrm{mmol}$ ), after flash chromatography with petroleum ether/ethyl acetate (10:1) compound 19 (150 mg, $0.16 \mathrm{mmol}, 68 \%$ ) was obtained as slightly yellow oil. $\mathrm{R}_{\mathrm{f}}=0.45$ (petroleum ether/ethyl acetate $=5: 1) ;[\alpha]_{\mathrm{D}}=+5.2\left(\mathrm{c}=1, \mathrm{CHCl}_{3}\right) .{ }^{1} \mathrm{H} \mathrm{NMR}\left(250 \mathrm{MHz}, \mathrm{CDCl}_{3}\right): \delta=8.20$ (s, $1 \mathrm{H}, 1 \mathrm{a}-\mathrm{H}), 7.37-7.24\left(\mathrm{~m}, 25 \mathrm{H}\right.$, arom. H), 5.44 (d, $\left.J_{1,2}=3.7 \mathrm{~Hz}, 1 \mathrm{H}, 1 \mathrm{~b}-\mathrm{H}\right), 5.25$ (dd, $J_{3,4}$ $\left.=3.4 \mathrm{~Hz}, J_{3,1}=1.3 \mathrm{~Hz}, 1 \mathrm{H}, 3 \mathrm{a}-\mathrm{H}\right), 4.86(\mathrm{~d}, J=11.41 \mathrm{~Hz}, 1 \mathrm{H}$, benzyl. H), 4.83-4.37 (m, $9 \mathrm{H}$, benzyl. H), 4.24-4.21 (m, 2 H, 4a-H, 5a-H), 4.05 (d, $J=4.8$ Hz, $1 \mathrm{H}, 5 \mathrm{~b}-\mathrm{H}), 4.02-3.89$ (m, 4 H, 2b-H, 4b-H, 6a-H, 6a'-H), 3.78 (dd, $\left.J_{3,2}=10.4 \mathrm{~Hz}, J_{3,4}=2.7 \mathrm{~Hz}, 1 \mathrm{H}, 3 \mathrm{~b}-\mathrm{H}\right), 3.69-3.66$ (m, $2 \mathrm{H}, 6 \mathrm{~b}-\mathrm{H}, 6 \mathrm{~b}^{\prime}-\mathrm{H}$ ), 1.05-1.00 (m, $21 \mathrm{H}$, TIPS). MALDI-MS (positive mode, DHB): [M + $\mathrm{Na}]+: \mathrm{m} / z$ calcd.: 982.4, found: 981.0. HRMS (ESI) $\mathrm{m} / z$ calcd for $\left[\mathrm{C}_{56} \mathrm{H}_{69} \mathrm{NO}_{11} \mathrm{Si}+\mathrm{K}\right]^{+}$ 998.4372, found 998.4268 .

20

$O$-(2,3,4,6-Tetra- $O$-benzyl- $\alpha$-D-galactopyranosyl)-( $\rightarrow \rightarrow 3)$-di-4,6- $O$-benzyl-2-nitro-D-

galactal (20). Following general procedure A from 18 (751 mg, $0.23 \mathrm{mmol})$, after flash chromatography with toluene/ethyl acetate (40:1) compound $20(501 \mathrm{mg}, 0.56 \mathrm{mmol}, 63 \%)$ was obtained as slightly yellow oil. $R_{\mathrm{f}}=0.48$ (petroleum ether/ethyl acetate $=5: 1$ ); $[\alpha]_{\mathrm{D}}=$ 
+91.4 $\left(\mathrm{c}=1, \mathrm{CHCl}_{3}\right) .{ }^{1} \mathrm{H}$ NMR $\left(250 \mathrm{MHz}, \mathrm{CDCl}_{3}\right): \delta=8.12(\mathrm{~s}, 1 \mathrm{H}, 1 \mathrm{a}-\mathrm{H}), 7.40-7.16(\mathrm{~m}, 30$ $\mathrm{H}$, arom. H), $5.56\left(\mathrm{~d}, J_{1,2}=3.6 \mathrm{~Hz}, 1 \mathrm{H}, 1 \mathrm{~b}-\mathrm{H}\right), 5.28\left(\mathrm{dd}, J_{3,4}=3.6 \mathrm{~Hz}, J_{3,1}=1.4 \mathrm{~Hz}, 1 \mathrm{H}, 3 \mathrm{a}-\right.$ $\mathrm{H}), 4.90(\mathrm{~d}, J=11.2 \mathrm{~Hz}, 1 \mathrm{H}$, benzyl. H), 4.81-4.49 (m, $11 \mathrm{H}$, benzyl. H), 4.15-3.85 (m, $7 \mathrm{H}$, 2b-H, 3a-H, 4a-H, 4b-H, 5a-H, 5b-H, 6a-H, 6a'-H), 3.72 (dd, $J_{3,2}=7.8 \mathrm{~Hz}, J_{3,4}=3.2 \mathrm{~Hz}, 1 \mathrm{H}$, 3b-H), 3.69-3.66 (d, $J=7.4 \mathrm{~Hz}, 2 \mathrm{H}, 6 \mathrm{~b}-\mathrm{H}, 6 \mathrm{~b}-\mathrm{H}) .{ }^{13} \mathrm{C} \mathrm{NMR}\left(63 \mathrm{MHz}, \mathrm{CDCl}_{3}\right): \delta=155.5(\mathrm{C}-$ 1a), 138.9-127.2 (36 C, arom. C), 97.6 (C-1b), 78.3 (C-3b), 78.0 (C-2b), 74.9, 74.9, 74.7, 74.0, 73.1, 72.9, 72.5, 69.4, 68.2, 67.9, 63.1 (3 C). MALDI-MS (positive mode, DHB): [M + $\mathrm{Na}$ ]+: $m / z$ calcd.: 916.4, found: 916.2. $\mathrm{C}_{54} \mathrm{H}_{55} \mathrm{NO}_{11}(894.01)$ calcd.: C: $72.55, \mathrm{H}: 6.20, \mathrm{~N}$ : 1.57, found: C: $72.62, \mathrm{H}: 6.22, \mathrm{~N}: 1.81$.

\section{1a}

$O$-(2,3,4,6-Tetra- $O$-benzyl- $\alpha$-D-galactopyranosyl)-( $1 \rightarrow 3)$-(di-4,6- $O$-benzyl-2-nitro-2deoxy- $\alpha$-D-galactopyranosyl)- $N$-( $t$-butyloxycarbonyl)-L-serine tert-butylester (21a). To a solution of $19(251 \mathrm{mg}, 0.24 \mathrm{mmol}), 7 \mathbf{a}(130 \mathrm{mg}, 0.48 \mathrm{mmol})$ and dry toluene $(5 \mathrm{~mL})$ was added potassium tert-butoxide $(3 \mathrm{mg}, 0.027 \mathrm{mmol})$ under stirring. After $2 \mathrm{~h}$ the reaction mixture was neutralised with acetic acid and the solution concentrated under reduced pressure. Purification by flash chromatography (toluene/ethyl acetate $=40: 1$ ) afforded 21a (161 mg, $0.14 \mathrm{mmol}, 58 \%$ ) as colourless oil. $\mathrm{R}_{\mathrm{f}}=0.68$ (petroleum ether/ethyl acetate $=3: 1$ ); $[\alpha]_{\mathrm{D}}=+39.2\left(\mathrm{c}=1, \mathrm{CHCl}_{3}\right) .{ }^{1} \mathrm{H} \mathrm{NMR}\left(600 \mathrm{MHz}, \mathrm{CDCl}_{3}\right): \delta=7.33-7.20(\mathrm{~m}, 30 \mathrm{H}$, arom. $\mathrm{H})$, $5.44(\mathrm{~d}, J=7.8 \mathrm{~Hz}, 1 \mathrm{H}, \mathrm{NH}), 5.22\left(\mathrm{~d}, J_{1,2}=3.8 \mathrm{~Hz}, 1 \mathrm{H}, 1 \mathrm{a}-\mathrm{H}\right), 5.16(\mathrm{~s}, 1 \mathrm{H}, 1 \mathrm{~b}-\mathrm{H}), 5.02$ (dd, $\left.J_{2,1}=3.8 \mathrm{~Hz}, J_{2,3}=8.7 \mathrm{~Hz}, 1 \mathrm{H}, 2 \mathrm{a}-\mathrm{H}\right), 4.91-4.88(\mathrm{~m}, 2 \mathrm{H}$, benzyl. H), $4.84(\mathrm{~d}, J=11.3 \mathrm{~Hz}, 2$ $\mathrm{H}$, benzyl. H), 4.86-4.60 (m, $3 \mathrm{H}$, benzyl.H), $4.56(\mathrm{t}, J=11.2 \mathrm{~Hz}, 2 \mathrm{H}$, benzyl. H), 4.50-4.44 (m, $3 \mathrm{H}, 3 \mathrm{a}-\mathrm{H}$, benzyl. H), 4.35 (d, $J=10.7 \mathrm{~Hz}, 1 \mathrm{H}$, benzyl H), 4.30-4.28 (m, $1 \mathrm{H}, \alpha-$ Ser-H), $4.13\left(\mathrm{dd}, J_{2,1}=3.2 \mathrm{~Hz}, J_{2,3}=10.1 \mathrm{~Hz}, 1 \mathrm{H}, 2 \mathrm{~b}-\mathrm{H}\right), 4.01(\mathrm{~s}, 3 \mathrm{H}, 4 \mathrm{a}-\mathrm{H}, 4 \mathrm{~b}-\mathrm{H}, 5 \mathrm{~b}-\mathrm{H}), 3.95-3.93$ (m, $1 \mathrm{H}, 5 \mathrm{a}-\mathrm{H}), 3.86-3.83$ (m, $3 \mathrm{H}, 3 \mathrm{~b}-\mathrm{H}, \beta$-Ser-H, $\beta^{\prime}-$ Ser-H), 3.66 (dd $J_{6,5}=J_{6,6^{\prime}}=8.8 \mathrm{~Hz}, 1$ $\mathrm{H}, 6 \mathrm{~b}-\mathrm{H}), 3.55-3.53$ (m, $\left.1 \mathrm{H}, 6 \mathrm{~b}^{\prime}-\mathrm{H}\right), 3.46-3.44(\mathrm{~m}, 1 \mathrm{H}, 6 \mathrm{a}-\mathrm{H}), 3.39-3.36\left(\mathrm{~m}, 1 \mathrm{H}, 6 \mathrm{a}^{\prime}-\mathrm{H}\right)$, 1.44, $1.42(2 \times \mathrm{s}, 18 \mathrm{H}, \mathrm{t}-\mathrm{Bu}, \mathrm{Boc}) .{ }^{13} \mathrm{C} \mathrm{NMR}\left(151 \mathrm{MHz}, \mathrm{CDCl}_{3}\right): \delta=138.8-127.4(36 \mathrm{C}$, arom. C), 98.3 (C-1b), 96.7 (C-1a), 84.6 (C-2a), 82.6, 79.1 (C-3b), 76.1 (C-2b), 74.9, 74.8, 74.6 (C-4b), 74.0 (C-3a), 73.4 (C-5a), 72.4, 70.0 (C-5b),69.6 (C- 3 ), 68.1 (C-6b), 68.0 (C-6a), $54.4(\mathrm{C}-\alpha)$, 28.3, 27.9 (11 C). MALDI-MS (positive mode, DHB): $[\mathrm{M}+\mathrm{Na}]+: \mathrm{m} / z$ calcd.: 1177.5, found: 1177.1. $\mathrm{C}_{66} \mathrm{H}_{78} \mathrm{~N}_{2} \mathrm{O}_{16}(1155.33)$ calcd.: $\mathrm{C}: 68.61, \mathrm{H}: 6.80, \mathrm{~N}: 2.42$, found: $\mathrm{C}$ : 67.84, H: 6.30, N: 2.41 .

\section{3a}

$O$-(2,3,4,6-Tetra- $O$-benzyl- $\alpha$-D-galactopyranosyl)-( $\rightarrow 3)$-(4- $O$-benzyl-2-nitro-2-deoxy-6$O$-(triisopropylsilyl)- $\alpha$-D-galactopyranosyl)- $N$-(t-butyloxycarbonyl)-L-serine tertbutylester (23a). To a solution of $20(60 \mathrm{mg}, 0.07 \mathrm{mmol}), 7 \mathbf{a}(39 \mathrm{mg}, 0.14 \mathrm{mmol})$ in dry toluene $(3 \mathrm{~mL})$ was added potassium tert-butoxide $(2.5 \mathrm{mg}, 0.021 \mathrm{mmol})$ under stirring. After $2 \mathrm{~h}$ the reaction mixture was neutralised with acetic acid and the solution concentrated under reduced pressure. Purification by flash chromatography (toluene/ethyl acetate $=40: 1$ ) afforded 23a $(70 \mathrm{mg}, 0.065 \mathrm{mmol}, 59 \%)$ as colourless oil. $\mathrm{R}_{\mathrm{f}}=0.59$ (toluene/ethyl acetate $=$ $10: 1) ;[\alpha]_{\mathrm{D}}=+43.4\left(\mathrm{c}=1, \mathrm{CHCl}_{3}\right) .{ }^{1} \mathrm{H}$ NMR $\left(250 \mathrm{MHz}, \mathrm{CDCl}_{3}\right): \delta=7.46-7.10(\mathrm{~m}, 25 \mathrm{H}$, arom. H), $5.23(\mathrm{~d}, J=8.2 \mathrm{~Hz}, 1 \mathrm{H}, \mathrm{NH}), 5.22\left(\mathrm{~d}, J_{1,2}=4.2 \mathrm{~Hz}, 1 \mathrm{H}, 1 \mathrm{a}-\mathrm{H}\right), 5.15\left(\mathrm{~d}, J_{1,2}=4.2\right.$ $\mathrm{Hz}, 1 \mathrm{H}, 1 \mathrm{~b}-\mathrm{H}), 5.02\left(\mathrm{dd}, J_{2,1}=4.2 \mathrm{~Hz}, J_{2,3}=10.8 \mathrm{~Hz}, 1 \mathrm{H}, 2 \mathrm{a}-\mathrm{H}\right), 5.00-4.80(\mathrm{~m}, 4 \mathrm{H}$, benzyl. $\mathrm{H})$, 4.65-4.49 (m, $7 \mathrm{H}, 3 \mathrm{a}-\mathrm{H}$, benzyl. H), 4.30-4.20 (m, $1 \mathrm{H}, \alpha-S e r-\mathrm{H}), 4.11\left(\mathrm{dd}, J_{2,1}=4.2 \mathrm{~Hz}\right.$, $\left.J_{2,3}=9.9 \mathrm{~Hz}, 1 \mathrm{H}, 2 \mathrm{~b}-\mathrm{H}\right), 4.09-4.01(\mathrm{~m}, 3 \mathrm{H}, 4 \mathrm{a}-\mathrm{H}, 4 \mathrm{~b}-\mathrm{H}, 5 \mathrm{~b}-\mathrm{H}), 3.86\left(\mathrm{dd}, J_{3,2}=9.9 \mathrm{~Hz}, J_{3,4}=\right.$ $4.1 \mathrm{~Hz}, 1 \mathrm{H}, 3 \mathrm{a}-\mathrm{H}), 3.83-3.50$ (m, 7 H, 5a-H, 6a-H, 6a'-H, 6b-H, 6b'-H, $\beta$-Ser-H, $\beta$ '-Ser-H), $1.45(2 \times \mathrm{s}, 18 \mathrm{H}, \mathrm{t}-\mathrm{Bu}, \mathrm{Boc}), 1.03-0.98$ (m, $21 \mathrm{H}$, TIPS). ${ }^{13} \mathrm{C} \mathrm{NMR}\left(151 \mathrm{MHz}, \mathrm{CDCl}_{3}\right): \delta=$ 168.5, 160.5, 137.3-125.9 (30 C, arom. C), 96.7 (C-1b), 95.1 (C-1a), 94.4, 83.2 (C-2a), 80.9, $77.5,74.4,73.3,73.2,73.1,72.8,72.6,71.8,70.9,68.4,66.5,60.2,59.3,52.6,28.6,28.4,18.0$ 
(2 C), 11.0 (13 C). MALDI-MS (positive mode, DHB): [M + Na]+: $\mathrm{m} / z$ calcd.: 1243.6, found: 1243.0. $\mathrm{C}_{68} \mathrm{H}_{92} \mathrm{~N}_{2} \mathrm{O}_{16} \mathrm{Si}(1221.55)$ calcd.: C: 66.86, H: 7.59, N: 2.29, found: C: 66.77, H: 7.81, $\mathrm{N}: 2.40$.

\section{3b}

$O$-(2,3,4,6-Tetra- $O$-benzyl- $\alpha$-D-galactopyranosyl)-( $\rightarrow 3)$-(4-O-benzyl-2-nitro-2-deoxy-6$O$-(triisopropylsilyl)- $\alpha$-D-galactopyranosyl)- $N$-(t-butyloxycarbonyl)-L-threonine tertbutylester (23b). To a solution of $20(100 \mathrm{mg}, 0.11 \mathrm{mmol}), 7 \mathbf{b}(58 \mathrm{mg}, 0.21 \mathrm{mmol})$ in dry toluene $(3 \mathrm{~mL})$ was added potassium tert-butoxide $(3 \mathrm{mg}, 0.011 \mathrm{mmol})$ under stirring. After 2 $\mathrm{h}$ the reaction mixture was neutralised with acetic acid and the solution concentrated under reduced pressure. Purification by flash chromatography (toluene/ethyl acetate $=30: 1$ ) afforded 23b (70 mg, 0,065 mmol, 59\%) as colourless oil. $\mathrm{R}_{\mathrm{f}}=0.51$ (toluene/ethyl acetate $=$ $10: 1) ;[\alpha]_{\mathrm{D}}=+55.3\left(\mathrm{c}=1, \mathrm{CHCl}_{3}\right) .{ }^{1} \mathrm{H}$ NMR $\left(600 \mathrm{MHz}, \mathrm{CDCl}_{3}\right): \delta=7.32-7.23(\mathrm{~m}, 25 \mathrm{H}$, arom. $\mathrm{H}), 5.31\left(\mathrm{~d}, J_{1,2}=4.2 \mathrm{~Hz}, 1 \mathrm{H}, 1 \mathrm{a}-\mathrm{H}\right), 5.13\left(\mathrm{~d}, J_{1,2}=3.6 \mathrm{~Hz}, 1 \mathrm{H}, 1 \mathrm{~b}-\mathrm{H}\right), 5.05-4.97(\mathrm{~m}, 2$ H, 2a-H, NH), 4.94 (d, $J=10.8 \mathrm{~Hz}, 1 \mathrm{H}$, benzyl. H), 4.89 (d, $J=11.4 \mathrm{~Hz}, 1 \mathrm{H}$, benzyl. H), $4.84(\mathrm{~d}, J=11.4 \mathrm{~Hz}, 1 \mathrm{H}$, benzyl. H), $4.67(\mathrm{~d}, J=10.8 \mathrm{~Hz}, 1 \mathrm{H}$, benzyl H), $4.57(\mathrm{~s}, 2 \mathrm{H}$, benzyl H), $4.55\left(\mathrm{~d}, J=10.8 \mathrm{~Hz}, 2 \mathrm{H}\right.$, benzyl. H), $4.50\left(\mathrm{dd}, J_{3,2}=8.4 \mathrm{~Hz}, J_{3,4}=3.6 \mathrm{~Hz}, 1 \mathrm{H}, 3 \mathrm{a}-\right.$ $\mathrm{H}), 4.45(\mathrm{~d}, J=12.0 \mathrm{~Hz}, 1 \mathrm{H}$, benzyl H), $4.43(\mathrm{~d}, J=11.4 \mathrm{~Hz}, 1 \mathrm{H}$, benzyl H), 4.3-4.29 (m, 1 $\mathrm{H}, \beta-\mathrm{Thr}-\mathrm{H}$ ), 4.13 (dd, $\left.J_{2,1}=3.6 \mathrm{~Hz}, J_{2,3}=10.2 \mathrm{~Hz}, 1 \mathrm{H}, 2 \mathrm{~b}-\mathrm{H}\right), 4.06-4.05$ (m, $2 \mathrm{H}, 4 \mathrm{a}-\mathrm{H}, \alpha-$ Thr-H), 4.00-3.98 (m, $2 \mathrm{H}, 4 \mathrm{~b}-\mathrm{H}, 5 \mathrm{~b}-\mathrm{H}), 3.86\left(\mathrm{dd}, J_{3,2}=10.2 \mathrm{~Hz}, J_{3,4}=3.0 \mathrm{~Hz}, 1 \mathrm{H}, 3 \mathrm{~b}-\mathrm{H}\right)$, 3.84 (dd, $J=7.8 \mathrm{~Hz}, 1 \mathrm{H}, 5 \mathrm{a}-\mathrm{H}), 3.69-3.66$ (m, 2-H, 6a-H, 6a'-H), 3.60-3.57 (m, $2 \mathrm{H}, 6 \mathrm{~b}-\mathrm{H}$, 6b'-H), 1.45, $144(2 \times \mathrm{s}, 18 \mathrm{H}, \mathrm{t}-\mathrm{Bu}, \mathrm{Boc}), 1.26$ (d, $J=7.2 \mathrm{~Hz}, 3 \mathrm{H}, \mathrm{Me}), 1.03-1.00(\mathrm{~m}, 21 \mathrm{H}$, TIPS). ${ }^{13} \mathrm{C}$ NMR (151 MHz, $\left.\mathrm{CDCl}_{3}\right): \delta=167.7,138.3-127.3$, (30 C, arom. C), 98.4 (C-1b), 96.6 (C-1a), 89.6, 85.2 (C-2a), 79.1 (C-3b), 76.1 (C-2b), 75.4 (C- $\beta$ ), 75.0, 74.7 (4b-C), 74.7 (C-3a), 73.7 (C-4a), 73.4, 72.4, 72.0 (C-5a), 70.0 (5b-C), 68.1 (C-6b), 66.2, 65.9, 64.4, 62.0 (C-6a), $58.6(\mathrm{C}-\alpha), 37.1,28.0$ (14 C), 18.0 (2 C). MALDI-MS (positive mode, DHB): [M + $\mathrm{Na}]+: \mathrm{m} / z$ calcd.: 1257.6 , found: 1257.7 . HRMS (ESI) $m / z$ calcd for $\left[\mathrm{C}_{69} \mathrm{H}_{94} \mathrm{~N}_{2} \mathrm{O}_{16} \mathrm{Si}+\mathrm{Na}\right]^{+}$ 1257.6265, found 1257.6269.

25A

$O$-(2-Azido-2-deoxy-3,4,6-tri- $O$-benzyl- $\beta$-D-glucopyranosyl)-(1 $\rightarrow 3$ )-6- $O$ -

(triisopropylsilyl)-D-galactal (25A). A solution of $\mathbf{2}(253 \mathrm{mg}, 0.89 \mathrm{mmol})$ and $\mathbf{1 0 A}(450 \mathrm{mg}$, $0.73 \mathrm{mmol}$ ) in $\mathrm{MeCN}$ was cooled to $-40{ }^{\circ} \mathrm{C}$ and zinc(II) triflate $(3 \mathrm{mg}, 0.03 \mathrm{eq})$ in $1 \mathrm{~mL}$ MeCN added dropwise. After 30 min solid $\mathrm{NaHCO}_{3}$ and $\mathrm{CH}_{2} \mathrm{Cl}_{2}$ was added and the suspension washed with water, dried with $\mathrm{MgSO}_{4}$, and then the solvent was evaporated in vacuo. Purification by flash chromatography (petroleum ether/ethyl acetate $=10: 1$ ) furnished 25A (338 mg, $0.45 \mathrm{mmol}, 61 \%$ ) as slightly yellow oil. $R_{\mathrm{f}}=0.75$ (petroleum ether/ethyl acetate $=3: 1) ;[\alpha]_{\mathrm{D}}\left(\mathrm{c}=0.1, \mathrm{CHCl}_{3}\right)=-24.6 .{ }^{1} \mathrm{H} \mathrm{NMR}\left(600 \mathrm{MHz}, \mathrm{CDCl}_{3}\right): \delta=7.39-7.6(\mathrm{~m}$, $15 \mathrm{H}$, arom. H), $6.43\left(\mathrm{dd}, J_{1,2}=6.6 \mathrm{~Hz}, J_{1,3}=1.8 \mathrm{~Hz} 1 \mathrm{H}, 1 \mathrm{a}-\mathrm{H}\right), 4.88(\mathrm{~d}, J=10.8 \mathrm{~Hz}, 1 \mathrm{H}$, benzyl. H), 4.79 (d, $J=10.8 \mathrm{~Hz}, 2 \mathrm{H}$, benzyl. H), 4.74-4.73 (m, $1 \mathrm{H}, 2 \mathrm{a}-\mathrm{H}), 4.59-4.54(\mathrm{~m}, 2$ $\mathrm{H}$, benzyl. H), 4.49-4.44 (m, $3 \mathrm{H}, 1 \mathrm{~b}-\mathrm{H}, 3 \mathrm{a}-\mathrm{H}$, benzyl. H), 4.19 (br s, $1 \mathrm{H}, 4 \mathrm{a}-\mathrm{H}), 4.06-4.03$ (m, $1 \mathrm{H}, 6 \mathrm{a}-\mathrm{H}), 3.94-3.92$ (m, $2 \mathrm{H}, 5 \mathrm{a}-\mathrm{H}, 6 \mathrm{a}-\mathrm{H}), 3.70-3.62$ (m, $3 \mathrm{H}, 3 \mathrm{~b}-\mathrm{H}, 6 \mathrm{~b}-\mathrm{H}, 6 \mathrm{~b}-\mathrm{H}), 3.50$ $\left(\mathrm{dd}, J_{2,1}=7.8 \mathrm{~Hz}, J_{2,3}=10.8 \mathrm{~Hz}, 1 \mathrm{H}, 2 \mathrm{~b}-\mathrm{H}\right), 3.47-3.40(\mathrm{~m}, 2 \mathrm{H}, 4 \mathrm{~b}-\mathrm{H}, 5 \mathrm{~b}-\mathrm{H}), 3.00(\mathrm{~s}, 1 \mathrm{H}$, $\mathrm{OH}), 1.13-1.01\left(\mathrm{~m}, 21 \mathrm{H}\right.$, TIPS). ${ }^{13} \mathrm{C}$ NMR $\left(151 \mathrm{MHz}, \mathrm{CDCl}_{3}\right): \delta=145.7(\mathrm{C}-1 \mathrm{a}), 137.9-$ 127.2 (24 C, arom. C), 101.7 (C-1b), 99.2 (C-2a), 83.2 (C-2b), 82.9 (C-5b), 77.4 (C-3b), 77.0 (C-5b), 75.6 (C-4b), 75.0 (C-3a), 73.6, 68.5 (C-6b), 66.1 (C-2b), 63.4 (C-4a), 61.8 (C-6a), 18.0 (2 C), 12.0 (2 C). MALDI-MS (positive mode, DHB): $[\mathrm{M}+\mathrm{Na}]+: \mathrm{m} / z$ calcd.: 782.4, found: 782.6. $\mathrm{C}_{42} \mathrm{H}_{57} \mathrm{~N}_{3} \mathrm{O}_{8} \mathrm{Si}(760.00)$ calcd.: $\mathrm{C}: 66.37, \mathrm{H}: 7.56, \mathrm{~N}: 5.53$, found: $\mathrm{C}: 66.05, \mathrm{H}$ : $7.57, \mathrm{~N}: 5.47$. 
$O$-(3,4,6-Tri- $O$-acetyl-2-deoxy-2-(2,2,2-trichlorethoxycarbonylamino)- $\beta$-Dglucopyranosyl)-(1 $\rightarrow \mathbf{3})$-6- $\boldsymbol{O}$-(triisopropylsilyl)-D-galactal $(\mathbf{2 5 B})$. A solution of $\mathbf{1 0 B}{ }^{[19]}(110$ $\mathrm{mg}, 0.17 \mathrm{mmol})$ and $2(100 \mathrm{mg}, 0.35 \mathrm{mmol})$ in $\mathrm{CH}_{2} \mathrm{Cl}_{2}(5 \mathrm{~mL})$ was cooled to $0{ }^{\circ} \mathrm{C}$ and zinc(II) triflate ( $4 \mathrm{mg}, 0.03 \mathrm{eq}$ ) in $0.25 \mathrm{~mL} \mathrm{MeCN}$ added dropwise within $15 \mathrm{~min}$. After 30 to $60 \mathrm{~min}$ (TLC-monitoring) the reaction was stopped by adding triethylamine. The organic phase was separated and the solvent removed under reduced pressure. Purification by flash chromatography (petroleum ether/ethyl acetate $=3: 1)$ furnished $25 B(80 \mathrm{mg}, 0.10 \mathrm{mmol}$, $62 \%)$ as slightly yellow oil. $R_{\mathrm{f}}=0.29$ (petroleum ether/ethyl acetate $\left.=3: 1\right) ;[\alpha]_{\mathrm{D}}(\mathrm{c}=1$, $\left.\mathrm{CHCl}_{3}\right)=-15.3 .{ }^{1} \mathrm{H} \mathrm{NMR}\left(250 \mathrm{MHz}, \mathrm{CDCl}_{3}\right): \delta=6,40\left(\mathrm{dd}, J_{1,2}=6.2 \mathrm{~Hz}, J_{1,3}=1.6 \mathrm{~Hz}, 1 \mathrm{H}\right.$, $1 \mathrm{a}-\mathrm{H}), 5.57(\mathrm{~d}, J=7.9 \mathrm{~Hz}, 1 \mathrm{H}, \mathrm{NH}), 5.36\left(\mathrm{dd}, J_{4,3}=J_{4,5}=9.4 \mathrm{~Hz}, 1 \mathrm{H}, 4 \mathrm{~b}-\mathrm{H}\right), 5.09\left(\mathrm{dd}, J_{3,2}=\right.$ $\left.J_{3,4}=9.4 \mathrm{~Hz}, 1 \mathrm{H}, 3 \mathrm{~b}-\mathrm{H}\right), 5.00\left(\mathrm{~d}, J_{1,2}=8.6 \mathrm{~Hz}, 1 \mathrm{H}, 1 \mathrm{~b}-\mathrm{H}\right), 4.73-4.63(\mathrm{~m}, 3 \mathrm{H}, 2 \mathrm{a}-\mathrm{H}$, $\mathrm{CH}_{2} \mathrm{CCl}_{3}$ ), 4.50 (br s, $\left.1 \mathrm{H}, 3 \mathrm{a}-\mathrm{H}\right), 4.29-4.01$ (m, $\left.4 \mathrm{H}, 6 \mathrm{a}-\mathrm{H}, 4 \mathrm{a}-\mathrm{H}, 6 \mathrm{~b}-\mathrm{H}, 6 \mathrm{~b}-\mathrm{H}\right), 3.99-3.85$ (m, $2 \mathrm{H}, 5 \mathrm{~b}-\mathrm{H}, 2 \mathrm{a}-\mathrm{H}), 3.67-3.60$ (m, $\left.2 \mathrm{H}, 5 \mathrm{a}-\mathrm{H}, 6 \mathrm{a}^{\prime}-\mathrm{H}\right), 3.31$ (br s, $\left.1 \mathrm{H}, \mathrm{OH}\right), 2.08,2.04,2.02$ (3 × s, $9 \mathrm{H}, 3 \times \mathrm{OAc}), 1.05-1.00\left(\mathrm{~m}, 21 \mathrm{H}\right.$, TIPS). $\mathrm{C}_{30} \mathrm{Cl}_{3} \mathrm{H}_{48} \mathrm{NO}_{13} \mathrm{Si}$ (765.14) calcd.: C: $47.69, \mathrm{H}:$ 6.32, N: 1.83, found: C: 47.84, H: 6.15, N: 1.80 .

\section{C}

$O$-(3.4,6-Tri- $O$-acetyl-2-(tetra-2,3,4,5-chlorphthalimido)-2-deoxy- $\beta$-D-glucopyranosyl)$(\mathbf{1} \rightarrow \mathbf{3})$-6-O-(triisopropylsilyl)-D-galactal $(\mathbf{2 5 C})$. A solution of $\mathbf{1 0 C} \mathbf{C}^{[20]}(371 \mathrm{mg}, 0.39 \mathrm{mmol})$ and 2 (111 mg, $0.52 \mathrm{mmol}$ ) in $\mathrm{CH}_{2} \mathrm{Cl}_{2}(5 \mathrm{~mL})$ was cooled to $0{ }^{\circ} \mathrm{C}$ and zinc(II) triflate $(38 \mu \mathrm{l}$ of a $0.1 \mathrm{M}$ solution in $\mathrm{MeCN}$ ) added dropwise within $15 \mathrm{~min}$. After 30 to $60 \mathrm{~min}$ (TLCmonitoring) the reaction was stopped by adding triethylamine. The organic phase was separated and the solvent removed in vacuo. Purification by flash chromatography (petroleum ether/ethyl acetate $=3: 1)$ furnished $25 \mathrm{C}(210 \mathrm{mg}, 0.24 \mathrm{mmol}, 63 \%)$ as colourless lyophilisate. $\mathrm{R}_{\mathrm{f}}=0.85$ (toluene/ethyl acetate $\left.=5: 1\right) ;[\alpha]_{\mathrm{D}}\left(\mathrm{c}=1, \mathrm{CHCl}_{3}\right)=-18.6 .{ }^{1} \mathrm{H} \mathrm{NMR}$ $\left(250 \mathrm{MHz}, \mathrm{CDCl}_{3}\right): \delta=6,45\left(\mathrm{~d}, J_{1,2}=4.8 \mathrm{~Hz}, 1 \mathrm{H}, 1 \mathrm{a}-\mathrm{H}\right), 5.67\left(\mathrm{dd}, J_{2,1}=8.4 \mathrm{~Hz}, J_{2,3}=9.1\right.$ $\mathrm{Hz}, 1 \mathrm{H}, 2 \mathrm{~b}-\mathrm{H}), 5.58\left(\mathrm{~d}, J_{1,2}=8.4 \mathrm{~Hz}, 1 \mathrm{H}, 1 \mathrm{~b}-\mathrm{H}\right), 5.20\left(\mathrm{dd}, J_{3,2}=9.1 \mathrm{~Hz}, J_{3,4}=10.0 \mathrm{~Hz}, 1 \mathrm{H}\right.$, 3b-H) 4.45-4.40 (m, 2 H, 2a-H, 4b-H), 4.37-4.28 (m, 2 H, 3a-H, 6b-H), 4.16-4.11 (m, 2 H, 5bH, 6b'-H), 4.00-3.96 (m, 1 H, 4a-H), 3.95-3.84 (m, 3 H, 5a-H, 6a-H, 6a'-H), 2.13, 2.10, 1.90 $(3 \times \mathrm{s}, 9 \mathrm{H}, 3 \times \mathrm{OAc}), 1.08-1.01(\mathrm{~m}, 21 \mathrm{H}$, TIPS). MALDI-MS (positive mode, DHB): [M + $\mathrm{Na}$ ]+: $m / z$ calcd.: 878.1, found: 878.0. $\mathrm{C}_{35} \mathrm{Cl}_{4} \mathrm{H}_{45} \mathrm{NO}_{13} \mathrm{Si}(857.63)$ calcd.: C: 49.02, H: 5.29, $\mathrm{N}$ : 1.63, found: C: 48.66, H: 5.32, N: 1.91 .

\section{6}

$O$-(2-Azido-2-deoxy-3,4,6-tri- $O$-benzyl- $\beta$-D-glucopyranosyl)-(1 $\rightarrow 3)-4-O$-benzyl-6- $O$ (triisopropylsilyl)-D-galactal (26). 25A (34 mg, $0.046 \mathrm{mmol})$ was dissolved in DMF (1 mL) and $\mathrm{NaH}(4 \mathrm{mg}, 0.1 \mathrm{mmol})$ added. After $15 \mathrm{~min}$ of stirring $\mathrm{BnBr}(0.12 \mathrm{~mL}, 1.0 \mathrm{mmol})$ was added dropwise. After termination of the reaction ethyl acetate $(10 \mathrm{~mL})$ was added and the organic phase washed with water $(3 \times 5 \mathrm{~mL})$. The organic phase was separated, dried with $\mathrm{MgSO}_{4}$ and concentrated in vacuo. Purification of the residue by flash chromatography (petroleum ether/ethyl acetate $=20: 1)$ furnished $26(22 \mathrm{mg}, 0.25 \mathrm{mmol}, 55 \%)$ as slightly yellow oil. $\mathrm{R}_{\mathrm{f}}=0.83$ (petroleum ether/ethyl acetate $\left.=3: 1\right) ;[\alpha]_{\mathrm{D}}\left(\mathrm{c}=1, \mathrm{CHCl}_{3}\right)=-33.8 .{ }^{1} \mathrm{H}$ $\operatorname{NMR}\left(600 \mathrm{MHz}, \mathrm{CDCl}_{3}\right): \delta=7.39-7.21\left(\mathrm{~m}, 20 \mathrm{H}\right.$, arom. H), $6.40\left(\mathrm{~d}, J_{1,2}=6.0 \mathrm{~Hz}, 1 \mathrm{H}, 1 \mathrm{a}-\right.$ $\mathrm{H}), 5.09$ (d, $J=11.8 \mathrm{~Hz}, 1 \mathrm{H}$, benzyl. H), $4.93(\mathrm{~d}, J=11.2 \mathrm{~Hz}, 1 \mathrm{H}$, benzyl. H), $4.85(\mathrm{~d}, J=$ $8.5 \mathrm{~Hz}, 3 \mathrm{H}, 2 \mathrm{a}-\mathrm{H}$, benzyl. H), 4.75 (d, $J=11.8 \mathrm{~Hz}, 1 \mathrm{H}$, benzyl. H), 4.62-4.59 (m, $3 \mathrm{H}, 3 \mathrm{a}-\mathrm{H}$, benzyl. H), 4.52 (d, $J=13.9 \mathrm{~Hz}, 1 \mathrm{H}$, benzyl. H), 4.48 (d, $\left.J_{1,2}=7.0,1 \mathrm{H}, 1 \mathrm{~b}-\mathrm{H}\right), 4.13$ (br s, 1 H, 4a-H), 4.04 (br s, $1 \mathrm{H}, 5 \mathrm{a}-\mathrm{H}), 3.88$ (d, $J=6.3 \mathrm{~Hz}, 2 \mathrm{H}, 6 \mathrm{a}-\mathrm{H}, 6 \mathrm{a}-\mathrm{H}), 3.79-3.78$ (m, $2 \mathrm{H}, 6 \mathrm{~b}-$ $\left.\mathrm{H}, 6 \mathrm{~b}^{\prime}-\mathrm{H}\right), 3.77$ (dd, $\left.J_{3,2}=J_{3,4}=8.5 \mathrm{~Hz}, 1 \mathrm{H}, 3 \mathrm{~b}-\mathrm{H}\right), 3.48-3.47(\mathrm{~m}, 3 \mathrm{H}, 2 \mathrm{~b}-\mathrm{H}, 3 \mathrm{~b}-\mathrm{H}, 5 \mathrm{~b}-\mathrm{H})$, 1.08-1.05 (m, $21 \mathrm{H}, \mathrm{TIPS}) .{ }^{13} \mathrm{C}$ NMR (151 MHz, $\mathrm{CDCl}_{3}$ ): $\delta=144.7$ (C-1a), 139.0- 127.3 (24 
C, arom. C), 101.2 (C-1b), 99.6 (C-2a), 83.2 (C-2b), 77.6 (C-3b), 77.6 (C-5a), 76.7, 75.5, 75.0, 74.9 (C-4b), 73.5, 72.9, 71.5, 68.8 (C-6b), 66.7 (C-5b), 61.7 (C-6a), 18.0 (3 C), 12.0 (7 C). MALDI-MS (positive mode, DHB): $[\mathrm{M}+\mathrm{Na}]^{+}: \mathrm{m} / z$ calcd.: 872.4, found: 872.4. $\mathrm{C}_{49} \mathrm{H}_{63} \mathrm{O}_{8} \mathrm{Si}$ (850.13), calcd.: C: 69.23, H: 7.47, N: 4.94, found: C: 69.43, H: 7.12, N: 4.34.

27

$O$-(2-Azido-2-deoxy-3,4,6-tri-O-benzyl- $\beta$-D-glucopyranosyl)-(1 $\rightarrow 3$ )-4-O-benzyl-2-deoxy2-nitro-6-O-(triisopropylsilyl)-D-galactal (27). Following general procedure A from 42 (150 $\mathrm{mg}, 0.18 \mathrm{mmol}$ ), after flash chromatography with toluene/ethyl acetate (10:1) compound 27 $(100 \mathrm{mg}, 0.11 \mathrm{mmol}, 62 \%)$ was obtained as slightly yellow oil. $\mathrm{R}_{\mathrm{f}}=0.76$ (petroleum ether/ethyl acetate $=3: 1) ;[\alpha]_{\mathrm{D}}\left(\mathrm{c}=0.5, \mathrm{CHCl}_{3}\right)=+46.0 .{ }^{1} \mathrm{H} \mathrm{NMR}\left(250 \mathrm{MHz}, \mathrm{CDCl}_{3}\right): \delta=$ $8.19(\mathrm{~s}, 1 \mathrm{H}, 1 \mathrm{aH}), 7.39-7.21\left(\mathrm{~m}, 20 \mathrm{H}\right.$, arom. H), $5.17\left(\mathrm{~d}, J_{3,4}=4.1 \mathrm{~Hz}, 1 \mathrm{H}, 3 \mathrm{a}-\mathrm{H}\right), 5.00(\mathrm{~d}, J$ $=12.1 \mathrm{~Hz}, 1 \mathrm{H}$, benzyl. H), 4.95-4.85 (m, $2 \mathrm{H}, 2 \mathrm{a}-\mathrm{H}$, benzyl. H), 4.83-4.79 (m, $2 \mathrm{H}$, benzyl. H), 4.60-4.51 (m, $2 \mathrm{H}$, benzyl. H), 4.43 (d, J=4.0 Hz, $2 \mathrm{H}$, benzyl. H), 4.34-4.26 (m, $1 \mathrm{H}, 5 \mathrm{a}-$ H), 4.03-3.92 (m, 3 H, 4a-H, 6a-H, 6a'-H), 3.73-3.63 (m, 2 H, 6b-H, 6b'-H), 3.61-3.58 (m, 2 $\mathrm{H}, 5 \mathrm{~b}-\mathrm{H}, 3 \mathrm{~b}-\mathrm{H}), 3.51$ (dd, $\left.J_{4,3}=J_{4,5}=9.1 \mathrm{~Hz}, 1 \mathrm{H}, 4 \mathrm{~b}-\mathrm{H}\right), 3.38\left(\mathrm{dd}, J_{2,1}=J_{2,3}=98.0 \mathrm{~Hz}, 1 \mathrm{H}\right.$, 2b-H), 1.05-1.00 (m, $21 \mathrm{H}$, TIPS). ${ }^{13} \mathrm{C}$ NMR (63 MHz, $\left.\mathrm{CDCl}_{3}\right): \delta=155.7$ (C-1a), 137.8-127.6 (24 C, arom. C), 102.2 (C-1b), 83.2 (C-2b), 77.6 (C-3b), 77.6 (C-5a), 76.7, 75.5, 75.0, 74.9 (C-4b), 73.5, 72.9, 71.5, 68.8 (C-6b), 66.7 (C-5b), 61.7 (C-6a), 18.0, 12.0 (9 C). MALDI-MS (positive mode, DHB): $[\mathrm{M}+\mathrm{Na}]^{+}: \mathrm{m} / z$ calcd.: 917.3, found: 916.3. $\mathrm{C}_{49} \mathrm{H}_{62} \mathrm{~N}_{4} \mathrm{O}_{10} \mathrm{Si}(895.12$ ) calcd.: C: $65.75, \mathrm{H}: 7.98, \mathrm{~N}: 6.26$, found: C: $65.47, \mathrm{H}: 7.91, \mathrm{~N}: 5.84$.

30

Di-3,4-O-benzyl-D-galactal (30). To a solution of $\mathbf{2 9}^{[21]}(2.5 \mathrm{~g}, 4.4 \mathrm{mmol})$ in $100 \mathrm{~mL}$ THF, TBAF in THF ( $6.6 \mathrm{~mL}, 1 \mathrm{M}$ solution) was added. After $6 \mathrm{~h}$ the solvent was removed and the residue purified by flash chromatography (toluene/ethyl acetate $=5: 1$ ) which furnished $\mathbf{3 0}$ $(1.0 \mathrm{~g}, 3.1 \mathrm{mmol}, 70 \%)$ as colourless lyophilisate. $\mathrm{R}_{\mathrm{f}}=0.22$ (toluene/ethyl acetate $\left.=5: 1\right) ;[\alpha]_{\mathrm{D}}$ $\left(\mathrm{c}=1, \mathrm{CHCl}_{3}\right)=-2.0 .{ }^{1} \mathrm{H} \mathrm{NMR}\left(250 \mathrm{MHz}, \mathrm{CDCl}_{3}\right): \delta=7.38-7.33(\mathrm{~m}, 10 \mathrm{H}$, arom. H$), 6.43$ $\left(\mathrm{dd}, J_{1,2}=6.2 \mathrm{~Hz}, J_{1,3}=1.1 \mathrm{~Hz}, 1 \mathrm{H}, 1-\mathrm{H}\right), 4.86\left(\mathrm{dd}, J_{2,1}=6.2 \mathrm{~Hz}, J_{2,3}=3.8 \mathrm{~Hz}, 1 \mathrm{H}, 2-\mathrm{H}\right)$, 4.85 (d, $J=11.4 \mathrm{~Hz}, 1 \mathrm{H}$, benzyl. H), 4.65 (d, $J=11.9 \mathrm{~Hz}, 1 \mathrm{H}$, benzyl. H), 4.73 (d, $J=11.3$ Hz, $2 \mathrm{H}$, benzyl. H), 4.65 (d, $J=11.3 \mathrm{~Hz}, 1 \mathrm{H}$, benzyl. H), 4.24-4.18 (m, $1 \mathrm{H}, 3-\mathrm{H}), 4.09-3.95$ (m, $2 \mathrm{H}, 5-\mathrm{H}, 6-\mathrm{H}), 3.77$ (dd, $\left.J_{6^{\prime}, 5}=4.9 \mathrm{~Hz}, J_{6^{\prime}, 6}=11.9 \mathrm{~Hz}, 1 \mathrm{H}, 6^{\prime}-\mathrm{H}\right), 2.49(\mathrm{~s}, 1 \mathrm{H}, \mathrm{OH})$. MALDI-MS (positive mode, DHB): $[\mathrm{M}+\mathrm{Na}]^{+}: \mathrm{m} / \mathrm{z}$ calcd.: 349.2 , found: $349.7 . \mathrm{C}_{20} \mathrm{H}_{22} \mathrm{O}_{4}$ (326.39) calcd.: C: 73.60, H: 6.79, found: C: 73.20, H: 6.69.

\section{1}

$O$-(2-Azido-2-deoxy-3,4,6-tri- $O$-benzyl- $\beta$-D-glucopyranosyl)-( $1 \rightarrow 6)$-3,4-di- $O$-benzyl-Dgalactal (31). A solution of $\mathbf{3 0}(175 \mathrm{mg}, 0.53 \mathrm{mmol})$ and $\mathbf{1 0 A}(220 \mathrm{mg}, 0.35 \mathrm{mmol})$ in $\mathrm{MeCN}$ was cooled to $-40{ }^{\circ} \mathrm{C}$ and zinc(II) triflate $(1.5 \mathrm{mg}, 0.01 \mathrm{eq})$ in $0.25 \mathrm{~mL} \mathrm{MeCN}$ added dropwise. After 30 min solid $\mathrm{NaHCO}_{3}$ and $\mathrm{CH}_{2} \mathrm{Cl}_{2}$ were added and the suspension washed with water. After drying with $\mathrm{MgSO}_{4}$ the solvent was removed in vacuo. Purification by flash chromatography furnished $31(160 \mathrm{mg}, 0.20 \mathrm{mmol}, 58 \%)$ (petroleum ether/ethyl acetate = $10: 1)$ as slightly yellow oil. $\mathrm{R}_{\mathrm{f}}=0.65$ (petroleum ether/ethyl acetate $\left.=5: 1\right) ;[\alpha]_{\mathrm{D}}(\mathrm{c}=0.5$, $\left.\mathrm{CHCl}_{3}\right)=-8.5 .{ }^{1} \mathrm{H} \mathrm{NMR}\left(600 \mathrm{MHz}, \mathrm{CDCl}_{3}\right): \delta=7.39-7.13(\mathrm{~m}, 25 \mathrm{H}$, arom. $\mathrm{H}), 6.17\left(\mathrm{~d}, J_{1,2}=\right.$ $6.3 \mathrm{~Hz}, 1 \mathrm{H}, 1 \mathrm{a}-\mathrm{H}), 4.89(\mathrm{~d}, J=10.8 \mathrm{~Hz}, 2 \mathrm{H}$, benzyl. H), $4.78(\mathrm{~d}, \mathrm{~J}=10.8 \mathrm{~Hz}, 1 \mathrm{H}$, benzyl. $\mathrm{H})$, 4.73-4.68 (m, $3 \mathrm{H}, 2 \mathrm{a}-\mathrm{H}$, benzyl. H), 4.54-4.30 (m, $4 \mathrm{H}$, benzyl. H), $4.29\left(\mathrm{~d}, J_{1,2}=7.2 \mathrm{~Hz}\right.$, $1 \mathrm{H}, 1 \mathrm{~b}-\mathrm{H}), 4.02-3.94$ (m, 3 H, 3a-H, 4a-H, 6a-H), 3.70-3.63 (m, 4 H, 4b-H, 6a'-H, 6b-H, 6b'$\mathrm{H}), 3.39-3.37$ (m, $4 \mathrm{H}, 2 \mathrm{~b}-\mathrm{H}, 3 \mathrm{~b}-\mathrm{H}, 5 \mathrm{a}-\mathrm{H}, 5 \mathrm{~b}-\mathrm{H}) .{ }^{13} \mathrm{C} \mathrm{NMR}$ (151 MHz, $\mathrm{CDCl}_{3}$ (selected data)): $\delta=102.3(\mathrm{C}-1 \mathrm{~b}), 95.3$ (C-1a), 83.1 (C-3b), 78.2 (C-3a), 77.6 (C-4b), 75.0 (C-5b), 73.3 (C4a), 68.4 (C-6b), 67.8 (C-6a), 66.7 (C-2b), 62.4 (C-2a). MALDI-MS (positive mode, DHB): 
$[\mathrm{M}+\mathrm{Na}]^{+}: \mathrm{m} / z$ calcd.: 806.3 , found: 806.5. HRMS (ESI) $\mathrm{m} / z$ calcd for $\left[\mathrm{C}_{47} \mathrm{H}_{49} \mathrm{~N}_{3} \mathrm{O}_{8}+\mathrm{Na}\right]^{+}$ 806.3412 , found 806.3414 .

32

$O$-(2-Azido-2-deoxy-3,4,6-tri- $O$-benzyl- $\beta$-D-glucopyranosyl)-(1 $\rightarrow$ 6)-3,4-di- $O$-benzyl-2deoxy-2-nitro-D-galactal (32). Following general procedure A from 31 (1.6 g, 2.06 mmol), after flash chromatography with toluene/ethyl acetate (10:1) compound $\mathbf{3 2}(1.4 \mathrm{~g}, 1.60 \mathrm{mmol}$, $78 \%$ ) was obtained as slightly yellow oil. $R_{\mathrm{f}}=0.60$ (petroleum ether/ethyl acetate $=5: 1$ ); $[\alpha]_{\mathrm{D}}$ $\left(\mathrm{c}=0.1, \mathrm{CHCl}_{3}\right)=-1.4 .{ }^{1} \mathrm{H} \mathrm{NMR}\left(600 \mathrm{MHz}, \mathrm{CDCl}_{3}\right): \delta=8.12(\mathrm{~s}, 1 \mathrm{H}, 1 \mathrm{a}-\mathrm{H}), 7.35-7.25(\mathrm{~m}$, $25 \mathrm{H}$, arom. H), $6.90(\mathrm{~s}, 1 \mathrm{H}, 3 \mathrm{a}-\mathrm{H}), 4.86(\mathrm{~d}, J=9.6 \mathrm{~Hz}, 2 \mathrm{H}$, benzyl. H), 4.81-4.77 (m, $3 \mathrm{H}$, 5a-H, benzyl. H), 4.64-4.47 (m, $6 \mathrm{H}$, benzyl. H), 4.32 (d, $J=12.6 \mathrm{~Hz}, 1 \mathrm{H}, 6 \mathrm{a}-\mathrm{H}), 4.26$ (d, $J_{1,2}$ $=8.4 \mathrm{~Hz}, 1 \mathrm{H}, 1 \mathrm{~b}-\mathrm{H}), 4.12\left(\mathrm{dd}, J_{6^{\prime}, 5}=3.0 \mathrm{~Hz}, J_{6^{\prime}, 6}=12.6 \mathrm{~Hz}, 1 \mathrm{H}, 6 \mathrm{~b}^{\prime}-\mathrm{H}\right), 3.90\left(\mathrm{dd}, J_{4,3}=5.4\right.$ $\left.\mathrm{Hz}, J_{4,5}=3.6 \mathrm{~Hz}, 1 \mathrm{H}, 4 \mathrm{a}-\mathrm{H}\right), 3.68-3.64\left(\mathrm{~m}, 3 \mathrm{H}, 4 \mathrm{~b}-\mathrm{H}, 6 \mathrm{~b}-\mathrm{H}, 6 \mathrm{~b}^{\prime}-\mathrm{H}\right), 3.43\left(\mathrm{dd}, J_{2,1}=8.4 \mathrm{~Hz}\right.$, $\left.J_{2,3}=9.6 \mathrm{~Hz}, 1 \mathrm{H}, 2 \mathrm{~b}-\mathrm{H}\right), 3.39-3.35(\mathrm{~m}, 2 \mathrm{H}, 3 \mathrm{~b}-\mathrm{H}, 5 \mathrm{~b}-\mathrm{H}) .{ }^{13} \mathrm{C}$ NMR $\left(151 \mathrm{MHz}, \mathrm{CDCl}_{3}\right.$ (selected data)): $\delta=102.9$ (C-1b), 83.1 (C-3b), 77.8 (C-5a), 77.5 (C-4b), 74.9 (C-5b), 73.2 (C-4a), 68.3 (C-6b), 68.0 (C-6a), 67.7 (C-2a), 66.2 (C-2b). MALDI-MS (positive mode, DHB): $[\mathrm{M}+\mathrm{Na}]+: \mathrm{m} / z$ calcd.: 851.3 , found: 852.2. HRMS (ESI) $m / z$ calcd for $\left[\mathrm{C}_{47} \mathrm{H}_{48} \mathrm{~N}_{4} \mathrm{O}_{10}\right.$ $+\mathrm{Na}]^{+} 851.3262$, found 851.3249 .

\section{3a}

$O$-(2-Azido-2-deoxy-3,4,6-tri- $O$-benzyl- $\beta$-D-glucopyranosyl)-(1 $\rightarrow 6)$-)3,4-di- $O$-benzyl-2deoxy-2-nitro-D-galactopyranosyl)- $N$-( $t$-butyloxycarbonyl)-L-serine tert-butylester (33a). To a mixture of $32(40 \mathrm{mg}, 0.05 \mathrm{mmol})$ and $7 \mathbf{a}(1 \mathrm{mg}, 0.06 \mathrm{mmol})$ in dry toluene $(10 \mathrm{~mL})$ were added potassium tert-butoxide $(0.5 \mathrm{mg}, 0.005 \mathrm{mmol})$ under stirring. After $2 \mathrm{~h}$ the mixture was neutralized with ethyl acetate and the solvent removed in vacuo. Purification by flash chromatography (toluene/ethyl acetate $=10: 1)$ furnished 33a $(35 \mathrm{mg}, 0,032 \mathrm{mmol}, 64 \%)$ as colourless oil. $\mathrm{R}_{\mathrm{f}}=0.20$ (toluene/ethyl acetate $\left.=10: 1\right) ;[\alpha]_{\mathrm{D}}\left(\mathrm{c}=0.13, \mathrm{CHCl}_{3}\right)=+1.0 .{ }^{1} \mathrm{H}$ NMR (600 MHz, $\left.\mathrm{CDCl}_{3}\right): \delta=7.35-7.14(\mathrm{~m}, 25 \mathrm{H}$, arom. H), 5.30-5.28 (m, $2 \mathrm{H}, 1 \mathrm{a}-\mathrm{H}, \mathrm{NH})$, $4.97\left(\mathrm{dd}, J_{2,1}=3.7 \mathrm{~Hz}, J_{2,3}=10.3 \mathrm{~Hz}, 1 \mathrm{H}, 2 \mathrm{a}-\mathrm{H}\right), 4.88-4.78(\mathrm{~m}, 3 \mathrm{H}$, benzyl. H), $4.79(\mathrm{~d}, J=$ $11.2 \mathrm{~Hz}, 1 \mathrm{H}$, benzyl. H), $4.72(\mathrm{~d}, J=4.8 \mathrm{~Hz}, 2 \mathrm{H}$, benzyl H), $4.61(\mathrm{~d}, J=8.4 \mathrm{~Hz}, 1 \mathrm{H}$, benzyl. H), 4.55-4.51 (m, 2 H, benzyl. H), 4.44-4.40 (m, 2 H, 3a-H, benzyl. H), 4.31 (br s, $2 \mathrm{H}, 1 \mathrm{~b}-\mathrm{H}$, $\alpha-S e r-H), 4.04$ (s, 1 H, 4a-H), 4.02 (m, 2 H, 5a-H, 6a-H), 3.91-3.86 (m, 2 H, $\beta$-Ser-H, $\beta$ '-SerH), 3.70-3.66 (m, 4 H, 5B-H, 6a'-H, 6b-H, 6b'-H), 3.40 (d, J=6.7 Hz, $3 \mathrm{H}, 2 \mathrm{~b}-\mathrm{H}, 3 \mathrm{~b}-\mathrm{H}, 4 \mathrm{~b}-$ $\mathrm{H}), 1.50,146(2 \times \mathrm{s}, 18 \mathrm{H}, \mathrm{t}-\mathrm{Bu}, \mathrm{Boc}) .{ }^{13} \mathrm{C} \mathrm{NMR}\left(151 \mathrm{MHz}, \mathrm{CDCl}_{3}\right): \delta=137.9-127.7(30 \mathrm{C}$, arom. C), 102.3 (C-1b), 97.0 (C-1a), 84.0 (C-2a), 83.0 (C-3b), 77.7 (C-5b), 75.5, 75.1 (C-4b), 75.0, 74.9 (C-3a), 73.5, 72.9, 72.8 (C-4a), 69.8 (C-5a), 69.7 ( $\beta-C), 68.5(\mathrm{C}-6 \mathrm{~b}), 67.6(\mathrm{C}-6 \mathrm{a})$, $66.5(\mathrm{C}-2 \mathrm{~b}), 54.3(\alpha-\mathrm{C}), 28.4,27.9(10 \mathrm{C})$. MALDI-MS (positive mode, DHB): $[\mathrm{M}+\mathrm{Na}]^{+}$: $m / z$ calcd.: 1112.5, found: 1112.1. HRMS (ESI) $m / z$ calcd for $\left[\mathrm{C}_{59} \mathrm{H}_{71} \mathrm{~N}_{5} \mathrm{O}_{15}+\mathrm{Na}^{+}\right.$ 1112.4939, found 1112.4884.

\section{4a}

$O$-(2-Actamido-2-deoxy-3,4,6-tri- $O$-benzyl- $\beta$-D-glucopyranosyl)-(1 $\rightarrow 6)$-)3,4-di- $O$-benzyl2-deoxy-2-nitro-D-galactopyranosyl)- $N$-(t-butyloxycarbonyl)-L-serine tert-butylester (34a). Nitroglycoside 33a $(160 \mathrm{mg}, 0.147 \mathrm{mmol})$ was treated with thioacetic acid $(6-8 \mathrm{~mL}$, distilled three times) at $\mathrm{rt}$ for $5 \mathrm{~d}$ and after completion of reaction, excess thioacetic acid was co-evaporated with toluene $(4 \times 5 \mathrm{~mL})$ and the residue purified by flash chromatography (toluene/acetone 5:1) to give compound 34a as colourless solid $(160 \mathrm{mg}, 85 \%) ; \mathrm{mp} 109^{\circ} \mathrm{C}$. $R_{f}$ 0.42 (toluene/acetone $3: 1)$. $[\alpha]_{\mathrm{D}}=+48.3\left(\mathrm{c}=1, \mathrm{CHCl}_{3}\right) .{ }^{1} \mathrm{H}$ NMR $\left(600 \mathrm{MHz}, \mathrm{CDCl}_{3}\right): \delta 7.32-$ $7.16(\mathrm{~m}, 25 \mathrm{H}$, aromatic), $6.20(\mathrm{~d}, J=7.2 \mathrm{~Hz}, 1 \mathrm{H},-\mathrm{NHAc}), 5.49(\mathrm{~d}, J=7.2 \mathrm{~Hz}, 1 \mathrm{H}$, NHBoc), 5.27 (d, $J=3.0 \mathrm{~Hz}, 1 \mathrm{H}, 1 \mathrm{a}-\mathrm{H}), 4.95(\mathrm{dd}, J=10.2,4.2 \mathrm{~Hz}, 1 \mathrm{H}, 2 \mathrm{a}-\mathrm{H}), 4.85$ (d, $J=$ 
$7.8 \mathrm{~Hz}, 1 \mathrm{H}, 1 \mathrm{~b}-\mathrm{H}), 4.82-4.77\left(\mathrm{~m}, 3 \mathrm{H},-\mathrm{OCH}_{2} \mathrm{Ph}\right), 4.70-4.66\left(\mathrm{~m}, 3 \mathrm{H},-\mathrm{OCH}_{2} \mathrm{Ph}\right), 4.46-4.45$ $\left(\mathrm{m}, 3 \mathrm{H},-\mathrm{OCH}_{2} \mathrm{Ph}\right), 4.37$ (br d, $\left.J=10.2 \mathrm{~Hz}, 1 \mathrm{H}, 3 \mathrm{a}-\mathrm{H}\right), 4.27$ (t, $\left.J=9.0 \mathrm{~Hz}, 1 \mathrm{H}, 3 \mathrm{~b}-\mathrm{H}\right), 4.23$ (br d, $J=3.0 \mathrm{~Hz}, 1 \mathrm{H}, \alpha-$ Ser-H), 4.97-3.97 (m, $1 \mathrm{H}, \beta-S e r-H), 3.93$ (br s, $1 \mathrm{H}, 4 \mathrm{a}-\mathrm{H}$ ), 3.90 (br $\mathrm{d}, J=6.6 \mathrm{~Hz}, 1 \mathrm{H}, 5 \mathrm{~b}-\mathrm{H}), 3.86-3.84(\mathrm{~m} .1 \mathrm{H}, 6 \mathrm{~b}-\mathrm{H}), 3.79-3.78\left(\mathrm{~m}, 1 \mathrm{H}, \beta^{\prime}-\mathrm{Ser}-\mathrm{H}\right), 3.74-3.68$ (m, $3 \mathrm{H}, 2 \mathrm{~b}-\mathrm{H}, 6 \mathrm{a}-\mathrm{H}, 6 \mathrm{a}-\mathrm{H}), 3.62$ (t, $\left.J=8.4 \mathrm{~Hz}, 2 \mathrm{H}, 4 \mathrm{~d}-\mathrm{H}, 6 \mathrm{~b}^{\prime}-\mathrm{H}\right), 3.54$ (br d, $J=8.4 \mathrm{~Hz}, 1$ H, 5a-H), 3.25 (q, $J=8.4 \mathrm{~Hz}, 1 \mathrm{H}, 2 \mathrm{~b}-\mathrm{H}), 1.86$ (s, $3 \mathrm{H},-\mathrm{NHAc}), 1.45$ (2 s, $18 \mathrm{H}, t$-butyl). ${ }^{13} \mathrm{C}$ NMR (151 MHz, $\left.\mathrm{CDCl}_{3}\right): \delta 170.6,168.7,155.6,138.6-127.6(\mathrm{~m}, 30 \mathrm{C}$, aromatic), $99.8(\mathrm{C}-1 \mathrm{~b})$, 96.5 (C-1a), 83.8 (C-2a), 83.1, 80.2, 80.1 (C-3b), 78.5 (C-4b), 75.1, 74.9 (C-3a), 74.8, 74.7, 74.6 (C-5a), 73.4 ( $\beta-C), 73.2,73.0$ (C-4a), 70.5 (C-5b), 68.8 (C-6a), 68.4 (C-6b), 57.8 (C-2b), $54.3(\alpha-\mathrm{C}), 29.7,28.4,27.9$. MALDI-MS (positive mode, DHB): $1129.1[\mathrm{M}+\mathrm{Na}]^{+}$. Anal. Calcd. for $\mathrm{C}_{61} \mathrm{H}_{75} \mathrm{~N}_{3} \mathrm{O}_{16}$ (1105.51): C, 66.23; H, 6.83; N, 3.80, found: $\mathrm{C}, 66.28 ; \mathrm{H}, 7.91 ; \mathrm{N}$, 3.85 .

\section{3b}

$O$-(2-Azido-2-deoxy-3,4,6-tri- $O$-benzyl- $\beta$-D-glucopyranosyl)-(1 $\rightarrow 6)$-)3,4-di- $O$-benzyl-2deoxy-2-nitro-D-galactopyranosyl)- $N$-( $t$-butyloxycarbonyl)-L-thteonine tert-butylester (33b). 32 (230 mg, $0.277 \mathrm{mmol})$ and $7 \mathbf{b}(84 \mathrm{mg}, 0.305 \mathrm{mmol})$ were dried under high vacuum and dissolved in dry toluene $(10 \mathrm{~mL})$ under argon. Potassium tert-butoxide $(112 \mu \mathrm{L}, 1 \mathrm{M}$ solution in THF) was added and the reaction stirred for $27 \mathrm{~h}$ at $\mathrm{rt}$. The reaction was acidified with acetic acid, concentrated and the residue was purified by flash chromatography (toluene/ethyl acetate $8: 1$ ) to give glycoside $\mathbf{3 3 b}$ as colourless oil $(244 \mathrm{mg}, 80 \%)$. $\mathrm{R}_{\mathrm{f}} 0.23$ (toluene/ethyl acetate 8:1). $[\alpha]_{\mathrm{D}}=+38.0\left(\mathrm{c}=1, \mathrm{CHCl}_{3}\right) .{ }^{1} \mathrm{H} \mathrm{NMR}\left(600 \mathrm{MHz}, \mathrm{CDCl}_{3}\right): \delta 7.37-$ $7.17(\mathrm{~m}, 25 \mathrm{H}$, aromatic)), $5.43(\mathrm{~d}, J=4.2 \mathrm{~Hz}, 1 \mathrm{H}, 1 \mathrm{a}-\mathrm{H}), 5.05(\mathrm{~d}, J=10.0 \mathrm{~Hz}, 1 \mathrm{H}, \mathrm{NHBoc})$, 4.99 (dd, $J=10.7,4.2 \mathrm{~Hz}, 1 \mathrm{H}, 2 \mathrm{a}-\mathrm{H}), 4.90$ (d, $\left.J=10.8 \mathrm{~Hz}, 1 \mathrm{H},-\mathrm{OCH}_{2} \mathrm{Ph}\right), 4.86-4.81$ (m, 3 $\left.\mathrm{H},-\mathrm{OCH}_{2} \mathrm{Ph}\right), 4.74\left(\mathrm{~m}, 2 \mathrm{H},-\mathrm{OCH}_{2} \mathrm{Ph}\right), 4.61-4.53\left(\mathrm{~m}, 3 \mathrm{H},-\mathrm{OCH}_{2} \mathrm{Ph}\right), 4.47$ (dd, $J=10.8,3.2$ $\mathrm{Hz}, 1 \mathrm{H}, 3 \mathrm{a}-\mathrm{H}), 4.46\left(\mathrm{~d}, J=12.0 \mathrm{~Hz}, 1 \mathrm{H}, \mathrm{OCH}_{2} \mathrm{Ph}\right), 4.39$ (br dd, $J=6.5,2.0 \mathrm{~Hz}, 1 \mathrm{H}, \beta$-ThreH), 4.29 (d, $J=7.8 \mathrm{~Hz}, 1 \mathrm{H}, 1 \mathrm{~b}-\mathrm{H}), 4.13$ (dd, $J=10.0,2.0 \mathrm{~Hz}, 1 \mathrm{H}, \alpha-$ Thre-H), 4.10 (t, $J=6.5$ $\mathrm{Hz}, 1 \mathrm{H}, 5 \mathrm{a}-\mathrm{H}), 4.05(\mathrm{~d}, J=3.0 \mathrm{~Hz}, 1 \mathrm{H}, 4 \mathrm{a}-\mathrm{H}), 4.01(\mathrm{dd}, J=10.0,6.5 \mathrm{~Hz}, 1 \mathrm{H}, 6 \mathrm{a}-\mathrm{H}), 3.73-$ 3.67 (m, 4 H, 6a'-H, 6b-H, 6b'-H, 4b-H), 3.44-3.40 (m, 3 H, 3b-H, 5b-H, 2b-H), 1.50, 1.49, (2 $\mathrm{s}, 18 \mathrm{H}, t$-butyl), 1.33 (d, $J=6.6 \mathrm{~Hz}, 3 \mathrm{H}, \gamma$-Thre-H). ${ }^{13} \mathrm{C}$ NMR $\left(151 \mathrm{MHz}, \mathrm{CDCl}_{3}\right): \delta 169.3$, 156.2, 137.9-127.8 (m, 30C, aromatic), 102.1 (C-1b), 96.1 (C-1a), 84.3 (C-2a), 83.0 (C-3b), 82.6, 79.9, 77.4 (C-4b), 75,6, 75.5, 75.9 (B-C), 75.1 (C-3a), 75.0 (C-5b), 73.5, 72.9 (C-4a), 69.8 (C-5a), 68.5 (C-6b), 68.0 (C-6a), 66.4 (C-2b), 58.5 ( $\alpha$-C), 28.4, 28.0, 18.7. MALDI-MS (positive mode, DHB): $1127[\mathrm{M}+\mathrm{Na}]^{+}, 1143[\mathrm{M}+\mathrm{K}]^{+}$. Anal. Calcd for $\mathrm{C}_{60} \mathrm{H}_{73} \mathrm{~N}_{5} \mathrm{O}_{15}$ (1103.51): C, 65.26; H, 6.66; N, 6.34, found: C, 65.29; H, 6.69; N, 6.38.

\section{4b}

$O$-(2-Actamido-2-deoxy-3,4,6-tri- $O$-benzyl- $\beta$-D-glucopyranosyl)-(1 $\rightarrow 6)$-)3,4-di- $O$-benzyl2-deoxy-2-nitro-D-galactopyranosyl)- $N$-(t-butyloxycarbonyl)-L-threonine tert-butylester (34b). 34b was prepared in $82 \%$ yield from 33b following the same procedure as for the preparation of 34a. $[\alpha]_{\mathrm{D}}=+38.8\left(\mathrm{c}=1, \mathrm{CHCl}_{3}\right) .{ }^{1} \mathrm{H} \mathrm{NMR}\left(600 \mathrm{MHz}, \mathrm{CDCl}_{3}\right): \delta$ 7.23-7.17 (m, $25 \mathrm{H}$, aromatic), $5.46(\mathrm{~d}, J=8.5 \mathrm{~Hz}, 1 \mathrm{H},-\mathrm{NHAc}), 5.36(\mathrm{~d}, J=4.2 \mathrm{~Hz}, 1 \mathrm{H}, 1 \mathrm{a}-\mathrm{H}), 4.93$ $(\mathrm{d}, J=9.5 \mathrm{~Hz}, 1 \mathrm{H},-\mathrm{NHBoc}), 4.86(\mathrm{dd}, J=10.8,4.2 \mathrm{~Hz}, 1 \mathrm{H}, 2 \mathrm{a}-\mathrm{H}), 4.81(\mathrm{~d}, J=10.8, \mathrm{~Hz}, 1$ $\left.\mathrm{H},-\mathrm{OCH}_{2} \mathrm{Ph}\right), 4.80\left(\mathrm{~d} J=11.0 \mathrm{~Hz}, 1 \mathrm{H},-\mathrm{OCH}_{2} \mathrm{Ph}\right), 4.77(\mathrm{~d}, J=8.1 \mathrm{~Hz}, 1 \mathrm{H}, 1 \mathrm{~b}-\mathrm{H}), 4.72-4.46$ $\left(\mathrm{m}, 8 \mathrm{H},-\mathrm{OCH}_{2} \mathrm{Ph}\right), 4.39$ (dd, $J=10.8,3.2 \mathrm{~Hz}, 1 \mathrm{H}, 3 \mathrm{a}-\mathrm{H}, 4.23$ (m, $1 \mathrm{H}, \beta$-Thre-H), 3.96 (t, $J$ $=6.0 \mathrm{~Hz}, 1 \mathrm{H}, 5 \mathrm{~b}-\mathrm{H}), 3.89-3.86(\mathrm{~m}, 3 \mathrm{H}, 4 \mathrm{a}-\mathrm{H}, 4 \mathrm{~b}-\mathrm{H}, 6 \mathrm{a}-\mathrm{H}), 3.78-3.79(\mathrm{~m}, 3 \mathrm{H}, \alpha-$ Thre-H, 6a'-H, 3b-H), 3.61 (t, $J=8.5 \mathrm{~Hz}, 1 \mathrm{H}, 2 \mathrm{~b}-\mathrm{H}), 3.59-3.57$ (m, $3 \mathrm{H}, 5 \mathrm{a}-\mathrm{H}, 6 \mathrm{~b}-\mathrm{H}, 6 \mathrm{~b}-\mathrm{H}), 2.26$ (s, 3 $\mathrm{H},-\mathrm{NHAc}), 1.46(2 \mathrm{~s}, 18 \mathrm{H}, t$-butyl $), 1.20\left(\mathrm{~d}, J=6.6 \mathrm{~Hz}, 3 \mathrm{H}, \gamma\right.$-Thre-H). ${ }^{13} \mathrm{C}$ NMR $(151$ $\left.\mathrm{MHz}, \mathrm{CDCl}_{3}\right): \delta 172.0,169.3,156.1,137.9-127.7(\mathrm{~m}, 30 \mathrm{C}$, aromatic), $100.2(\mathrm{C}-1 \mathrm{~b}), 96.3(\mathrm{C}-$ 1a), 84.2 (C-2a), 82.8, 81.2 (C-4a), 79.9, 77.6, 76.3 (C-5a), 75.5 ( $\beta-C), 75.1$ (C-3a, C-2b), 
75.0, 74.9, 73.5, 73.1 (C-4b), 72.9, 70.1 (C-5b), 69.0, 68.6 (C-6a) 68.1 (C-6b), 63.4, $58.6(\alpha-$ C), 28.3, 27.9,21.0, 18.7. MALDI-MS (positive mode, DHB): $1143.1[\mathrm{M}+\mathrm{Na}]^{+}$. Anal. Calcd for $\mathrm{C}_{62} \mathrm{H}_{77} \mathrm{~N}_{3} \mathrm{O}_{16}(1119.53)$ : C, 66.47; $\mathrm{H}, 6.93 ; \mathrm{N}, 3.75$, found: $\mathrm{C}, 66.51 ; \mathrm{H}, 6.97 ; \mathrm{N}, 3.81$.

37

1,5-Anhydro-3,4-di-O-benzyl-6-O-tert-butyldiphenylsilyl-2-deoxy-2-nitro-D-lyxo-hex-1enitol (37). Following general procedure A from $\mathbf{3 6}^{[21]}(30 \mathrm{~g}, 0.053 \mathrm{~mol})$, after flash chromatography with toluene/ethyl acetate (98:2) compound $37(27 \mathrm{~g}, 84 \%)$ was obtained as light yellow oil. $\mathrm{R}_{\mathrm{f}}=0.46$ (toluene); $[\alpha]_{\mathrm{D}}{ }^{25}=-7.5\left(\mathrm{c}=12, \mathrm{CHCl}_{3}\right) .{ }^{1} \mathrm{H} \mathrm{NMR}(600 \mathrm{MHz}$, $\left.\mathrm{CDCl}_{3}\right): \delta 7.77(\mathrm{~s}, 1 \mathrm{H}, 1-\mathrm{H}), 7.61-7.60(\mathrm{~m}, 3 \mathrm{H}$, arom. H), 7.41-7.40 (m, $2 \mathrm{H}$, arom. H), 7.34$7.14\left(\mathrm{~m}, 15 \mathrm{H}\right.$, arom. H), $4.78\left(\mathrm{~d},{ }^{3} J_{3,4}=3.5 \mathrm{~Hz}, 1 \mathrm{H}, 3-\mathrm{H}\right), 4.75\left(\mathrm{~d},{ }^{2} J=10.9 \mathrm{~Hz}, 1 \mathrm{H}\right.$, benzyl H), $4.68\left(\mathrm{~d},{ }^{2} J=10.9 \mathrm{~Hz}, 1 \mathrm{H}\right.$, benzyl. H), 4.59-4.57 (m, $2 \mathrm{H}, 5-\mathrm{H}$, benzyl. H), 4.51 (d, ${ }^{2} J=$ $12.0 \mathrm{~Hz}, 1 \mathrm{H}$, benzyl. H), 4.22-4.14 (m, $2 \mathrm{H}, 6-\mathrm{H}, 6 \mathrm{-}-\mathrm{H}), 3.81\left(\mathrm{t},{ }^{3} J_{4,3}=4.6 \mathrm{~Hz},{ }^{3} J_{4,5}=4.6 \mathrm{~Hz}\right.$, $1 \mathrm{H}, 4 . \mathrm{H}), 1.05\left(\mathrm{~s}, 9 \mathrm{H}, \mathrm{C}_{4} \mathrm{H}_{9}\right) .{ }^{13} \mathrm{C} \mathrm{NMR}\left(150.8 \mathrm{MHz}, \mathrm{CDCl}_{3}\right): \delta 154.6(1-\mathrm{C}), 138.1,136.9$, 135.6 (2 C), 133.5, 133.3, 131.3, 129.8, 129.7, 128.6-127.4 (15 C), 95.7 (2-C), 80.1 (5-C), 74.6, 73.1 (4-C), 71.967 .4 (3-C), 61.5 (6-C), 26.9 (3 C). MS (FAB): calcd.: $609+1(\mathrm{H})=$ $610,609+23(\mathrm{Na})=632$; found: $610[\mathrm{M}+\mathrm{H}]^{+}, 632[\mathrm{M}+\mathrm{Na}]^{+} . \mathrm{C}_{36} \mathrm{H}_{39} \mathrm{NO}_{6} \mathrm{Si}(609.26)$. The mass peak could not be obtained by ESI HRMS.

\section{$38 \mathbf{a}$}

$O$-(3,4-Di-O-benzyl-6-O-tert-butyldiphenylsilyl-2-deoxy-2-nitro- $\alpha$-D-galactopyranosyl)$\mathrm{N}$-(tert-butyloxycarbonyl)-L-serine tert-butyl ester (38a). $37(0.02 \mathrm{~g}, 0.032 \mathrm{mmol})$ and 39a $(0.0231 \mathrm{~g}, 0.038 \mathrm{mmol})$ were dissolved in dry toluene $(1 \mathrm{~mL})$ and the reaction started by addition of potassium tert-butoxide solution $(30 \mu \mathrm{L}$ of a $0.1 \mathrm{M}$ solution in THF). After $90 \mathrm{~min}$ the amount of a product formed during the reaction did not increase any further. The reaction was quenched by addition of acetic acid and then concentrated. The residue was purified by column chromatography (toluene/ethyl acetate 20:1) to furnish 38a as colourless oil $(0.005 \mathrm{~g}$, $13 \%) . \mathrm{R}_{\mathrm{f}}=0.50$ (toluene/ethyl acetate $\left.10: 1\right) ;[\alpha]_{\mathrm{D}}{ }^{25}=+55.2\left(\mathrm{c}=5, \mathrm{CHCl}_{3}\right) .{ }^{1} \mathrm{H}$ NMR $(600$ $\left.\mathrm{MHz}, \mathrm{CDCl}_{3}\right): \delta$ 7.63-7.60 (m, $4 \mathrm{H}$, arom. $\left.\mathrm{H}\right), 7.43-7.15(\mathrm{~m}, 16 \mathrm{H}$, arom. $\mathrm{H})$, 5.23-5.21 (m, $\left.{ }^{3} J_{1,2}=4.2 \mathrm{~Hz}, 2 \mathrm{H}, 1-\mathrm{H}, \mathrm{NH}\right), 4.94\left(\mathrm{dd},{ }^{3} J_{2,1}=4.2 \mathrm{~Hz},{ }^{3} J_{2,3}=10.7 \mathrm{~Hz}, 1 \mathrm{H}, 2-\mathrm{H}\right), 4.83-4.73$ $\left(\mathrm{m}, 3 \mathrm{H}\right.$, benzyl. H), $4.46\left(\mathrm{~d},{ }^{2} J=11.0 \mathrm{~Hz}, 1 \mathrm{H}\right.$, benzyl. H), $4.41\left(\mathrm{dd},{ }^{3} J_{3,2}=10.7 \mathrm{~Hz},{ }^{3} J_{3,4}=\right.$ $2.9 \mathrm{~Hz}, 1 \mathrm{H}, 3-\mathrm{H}), 4.30-4.29(\mathrm{~m}, 1 \mathrm{H}, \alpha-\mathrm{H}), 3.99\left(\mathrm{~d},{ }^{3} J_{4,3}=2.7 \mathrm{~Hz}, 1 \mathrm{H}, 4-\mathrm{H}\right), 3.84-3.73(\mathrm{~m}, 5$ $\left.\mathrm{H}, 5-\mathrm{H}, 6-\mathrm{H}, 66^{\prime}-\mathrm{H}, \beta-\mathrm{H}, \beta^{\prime}-\mathrm{H}\right), 1.46,1.43\left(2 \mathrm{~s}, 18 \mathrm{H}, 2 \mathrm{C}_{4} \mathrm{H}_{9}\right), 1.05$ (s, $\left.9 \mathrm{H}, \mathrm{C}_{4} \mathrm{H}_{9}\right) .{ }^{13} \mathrm{C} \mathrm{NMR}$ $\left(150.8 \mathrm{MHz}, \mathrm{CDCl}_{3}\right): \delta 168.8,137.9-127.7$ (24 C), 96.8 (1-C), 84.1 (2-C), 82.7, 79.9, 75.1, 75.0 (3-C), 73.2, 73.1 (4-C), 71.5 (5-C), 69.3 ( $\beta-\mathrm{C}), 61.8$ (6-C), $54.1(\alpha-\mathrm{C}), 28.3$ (3 C), 27.9 (3 C), 26.9 (3 C). MS (FAB): calcd.: $870+23(\mathrm{Na})=893$; found: $893\left[\mathrm{M}+\mathrm{Na}^{+}\right.$. $\mathrm{C}_{48} \mathrm{H}_{62} \mathrm{~N}_{2} \mathrm{O}_{11} \mathrm{Si} \times 0.5 \mathrm{H}_{2} \mathrm{O}$ (880.12): calcd.: $\mathrm{C}$ 65.51, H 7.21, N 3.18, found: C 65.51, H 7.11, N 3.06 .

\section{$38 b$}

$O$-(3,4-Di- $O$-benzyl-6-O-tert-butyldiphenylsilyl-2-deoxy-2-nitro- $\alpha$-D-galactopyranosyl)$\mathrm{N}$-(tert-butyloxycarbonyl)-L-threonine tert-butyl ester (38b). 37 (13 g, $21.3 \mathrm{mmol})$ and $\mathbf{7 b}$ $(7.1 \mathrm{~g}, 25.6 \mathrm{mmol})$ were dried under high vacuum and dissolved in dry toluene $(250 \mathrm{~mL})$ under argon. Then potassium tert-butoxide solution ( $2.1 \mathrm{~mL}$ of a $1 \mathrm{M}$ solution in THF) was added and stirring continued for $2 \mathrm{~h}$. Acetic acid $(2 \mathrm{~mL})$ was used to neutralize the reaction mixture and all solvents were removed. The residue was purified by column chromatography (toluene/ethyl acetate $20: 1)$ to furnish $\mathbf{3 8 b}$ as colourless oil $(18.3 \mathrm{~g}, 97 \%) . \mathrm{R}_{\mathrm{f}}=0.57$ (toluene/ethyl acetate 10:1); $[\alpha]_{\mathrm{D}}{ }^{25}=+53.3\left(\mathrm{c}=5, \mathrm{CHCl}_{3}\right) .{ }^{1} \mathrm{H}$ NMR $\left(600 \mathrm{MHz}, \mathrm{CDCl}_{3}\right): \delta$ 7.61-7.60 (m, $4 \mathrm{H}$, arom. H), 7.39-7.18 (m, $16 \mathrm{H}$, arom. H), $5.32\left(\mathrm{~d},{ }^{3} J_{1,2}=4.4 \mathrm{~Hz}, 1 \mathrm{H}, 1-\mathrm{H}\right)$, $4.96\left(\mathrm{~d},{ }^{3} J_{\mathrm{NH}, \alpha}=9.7 \mathrm{~Hz}, 1 \mathrm{H}, \mathrm{NH}\right), 4.93\left(\mathrm{dd},{ }^{3} J_{2,1}=4.1,{ }^{3} J_{2,3}=10.6 \mathrm{~Hz}, 1 \mathrm{H}, 2-\mathrm{H}\right), 4.83\left(\mathrm{~d},{ }^{2} J\right.$ 
$=11.0 \mathrm{~Hz}, 1 \mathrm{H}$, benzyl. H), $4.77\left(\mathrm{~d},{ }^{2} J=11.0 \mathrm{~Hz}, 1 \mathrm{H}\right.$, benzyl. $\left.\mathrm{H}\right), 4.73\left(\mathrm{~d},{ }^{2} J=11.0 \mathrm{~Hz}, 1 \mathrm{H}\right.$, benzyl. H), $4.50\left(\mathrm{~d},{ }^{2} J=11.1 \mathrm{~Hz}, 1 \mathrm{H}\right.$, benzyl. H), $4.43\left(\mathrm{dd},{ }^{3} J_{3,2}=10.6,{ }^{3} J_{3,4}=2.9 \mathrm{~Hz}, 1 \mathrm{H}, 3-\right.$ $\mathrm{H}$ ), 4.24-4.23 (br d, $1 \mathrm{H}, \beta-\mathrm{H}), 4.06-4.05(\mathrm{~m}, 2 \mathrm{H}, \alpha-\mathrm{H}, 4-\mathrm{H}), 3.88$ (br t, ${ }^{3} J_{5,6}=6.8,{ }^{3} J_{5,6^{\prime}}=6.8$ $\mathrm{Hz}, 1 \mathrm{H}, 5-\mathrm{H}), 3.74\left(\mathrm{dd},{ }^{3} J_{6,5}=7.6,{ }^{2} J_{6,6^{6}}=10.3 \mathrm{~Hz}, 1 \mathrm{H}, 6-\mathrm{H}\right), 3.68\left(\mathrm{dd},{ }^{3} J_{6,5}=5.9,{ }^{2} J_{6,6}=10.0\right.$ $\mathrm{Hz}, 1 \mathrm{H}, 6$ '-H) $1.49,1.45\left(2 \mathrm{~s}, 18 \mathrm{H}, 2 \mathrm{C}_{4} \mathrm{H}_{9}\right), 1.04\left(\mathrm{~s}, 9 \mathrm{H}, \mathrm{C}_{4} \mathrm{H}_{9}\right) .{ }^{13} \mathrm{C}$ NMR $(150.8 \mathrm{MHz}$,

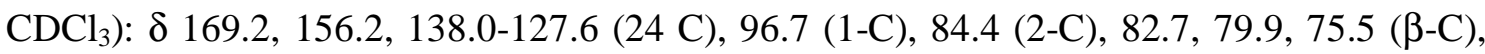
75.2, 75.2 (3-C), 73.3, 73.1 (4-C), 71.4 (5-C), 61.9 (6-C), $58.6(\alpha-C), 28.4(3 \mathrm{C}), 27.9$ (3 C), $26.8(3 \mathrm{C}), 18.9(\gamma-\mathrm{C})$. MS (FAB): calcd:: $884+23(\mathrm{Na})=907$; found: $907[\mathrm{M}+\mathrm{Na}]^{+}$. $\mathrm{C}_{49} \mathrm{H}_{64} \mathrm{~N}_{2} \mathrm{O}_{11} \mathrm{Si} \times 2 \mathrm{H}_{2} \mathrm{O}(921.17)$ calcd.: $\mathrm{C} 63.89, \mathrm{H} 7.44, \mathrm{~N} 3.04$, found: $\mathrm{C} 63.74, \mathrm{H} 7.35, \mathrm{~N}$ 3.26 .

\section{9a}

$O$-(3,4-Di- $O$-benzyl-2-deoxy-2-nitro- $\alpha$-D-galactopyranosyl)- $\mathrm{N}$-(tert-butyloxycarbonyl)-Lserine tert-butyl ester (39a). 38a $(0.5 \mathrm{~g}, 0.57 \mathrm{mmol})$ was dissolved in dry THF $(5 \mathrm{~mL})$ and treated with tetrabutylammonium fluoride $(1.10 \mathrm{~mL}$ of a $1 \mathrm{M}$ solution in THF) and acetic acid $(0.031 \mathrm{~mL}, 0.55 \mathrm{mmol})$. The reaction was stirred for $2 \mathrm{~h}$ at $\mathrm{rt}$ and then diluted with ethyl acetate and water. The organic layer was dried over sodium sulfate and concentrated. Purification by flash chromatography (toluene/ethyl acetate 3:1) furnished 39a as colourless foam $(0.351 \mathrm{~g}, 97 \%) . \mathrm{R}_{\mathrm{f}}=0.38$ (toluene/ethyl acetate $\left.2: 1\right) ;[\alpha]_{\mathrm{D}}{ }^{25}=+77.5\left(\mathrm{c}=1 \mathrm{CHCl}_{3}\right) .{ }^{1} \mathrm{H}$ NMR (600 MHz, $\left.\mathrm{CDCl}_{3}\right): \delta 7.38-7.25\left(\mathrm{~m}, 10 \mathrm{H}\right.$, arom. H), $5.56\left(\mathrm{~d},{ }^{3} J_{\mathrm{NH}, \alpha}=7.9 \mathrm{~Hz}, 1 \mathrm{H}, \mathrm{NH}\right)$, $5.31\left(\mathrm{~d},{ }^{3} J_{1,2}=4.4 \mathrm{~Hz}, 1 \mathrm{H}, 1-\mathrm{H}\right), 4.99\left(\mathrm{dd},{ }^{3} J_{2,1}=4.4,{ }^{3} J_{2,3}=10.9 \mathrm{~Hz}, 1 \mathrm{H}, 2-\mathrm{H}\right), 4.88\left(\mathrm{~d},{ }^{2} J=\right.$ 11.4 Hz, $1 \mathrm{H}$, benzyl. H), 4.77-4.73 (m, $2 \mathrm{H}$, benzyl. H), $4.53\left(\mathrm{~d},{ }^{2} \mathrm{~J}=11.4 \mathrm{~Hz}, 1 \mathrm{H}\right.$, benzyl. $\mathrm{H}), 4.37\left(\mathrm{dd},{ }^{3} J_{3,2}=10.6,{ }^{3} J_{3,4}=2.9 \mathrm{~Hz}, 1 \mathrm{H}, 3-\mathrm{H}\right), 4.30-4.29(\mathrm{~m}, 1 \mathrm{H}, \alpha-\mathrm{H}), 3.95-3.93(\mathrm{~m}, 2$ $\mathrm{H}, \beta-\mathrm{H}, 4-\mathrm{H}), 3.89-3.87(\mathrm{~m}, 2 \mathrm{H}, \beta-\mathrm{H}, 5-\mathrm{H}), 3.75\left(\mathrm{~d},{ }^{3} J_{6,5}=6.8,{ }^{2} J_{6,6^{\prime}}=11.4 \mathrm{~Hz}, 1 \mathrm{H}, 6-\mathrm{H}\right)$, $3.54\left(\mathrm{~d},{ }^{3} J_{6,5}=5.3,{ }^{2} J_{6,6^{\prime}}=11.4 \mathrm{~Hz}, 1 \mathrm{H}, 6 \mathrm{6}^{\prime} \mathrm{H}\right), 1.47,1.45\left(2 \mathrm{~s}, 18 \mathrm{H}, 2 \mathrm{C}_{4} \mathrm{H}_{9}\right) .{ }^{13} \mathrm{C} \mathrm{NMR}$ $\left(150.8 \mathrm{MHz}, \mathrm{CDCl}_{3}\right): \delta 168.8,137.5,137.1,128.6-128.2$ (10 C), 97.2 (1-C), 84.0 (2-C), 82.7, 79.9, 75.1 (3-C), 74.8, 73.3, 72.8 (4-C), $71.6(5-\mathrm{C}), 70.4$ ( $\beta-\mathrm{C}), 61.9(6-\mathrm{C}), 54.4(\alpha-\mathrm{C}), 28.3(3$ C), 27.9 (3 C). MS (FAB): calcd.: $632+23(\mathrm{Na})=655$; found: $655[\mathrm{M}+\mathrm{Na}]^{+}$. HRMS (ESI) $m / z$ calcd for $\left[\mathrm{C}_{31} \mathrm{H}_{44} \mathrm{~N}_{2} \mathrm{O}_{11}+\mathrm{Na}\right]^{+} 655.2837$, found 655.2818 .

\section{9b}

$O$-(3,4-Di- $O$-benzyl-2-deoxy-2-nitro- $\alpha$-D-galactopyranosyl)- $\mathrm{N}$-(tert-butyloxycarbonyl)-Lthreonine tert-butyl ester (39b). 38b (15.0 g, $16.9 \mathrm{mmol})$ was dissolved in dry THF (100 $\mathrm{mL}$ ) and treated with tetrabutylammonium fluoride $(51.0 \mathrm{~mL}$ of a $1 \mathrm{~m}$ solution in THF) and acetic acid $(1.4 \mathrm{~mL}, 25.4 \mathrm{mmol})$. The reaction was stirred for $2 \mathrm{~h}$ at $\mathrm{rt}$ and then diluted with ethyl acetate and water. The organic layer was dried over sodium sulfate and concentrated. Purification by flash chromatography (toluene/ethyl acetate 3:1) furnished 39a as colourless oil $(8.9 \mathrm{~g}, 97 \%) . \mathrm{R}_{\mathrm{f}}=0.60$ (toluene/ethyl acetate $\left.10: 1\right) ;[\alpha]_{\mathrm{D}}{ }^{25}=+56.3\left(\mathrm{c}=3, \mathrm{CHCl}_{3}\right) .{ }^{1} \mathrm{H}$ NMR $\left(600 \mathrm{MHz}, \mathrm{CDCl}_{3}\right): \delta 7.36-7.25\left(\mathrm{~m}, 10 \mathrm{H}\right.$, arom. H), $5.48\left(\mathrm{~d},{ }^{3} J_{1,2}=4.1 \mathrm{~Hz}, 1 \mathrm{H}, 1-\mathrm{H}\right)$, $5.10\left(\mathrm{~d},{ }^{3} J_{\mathrm{NH}, \alpha}=9.6 \mathrm{~Hz}, 1 \mathrm{H}, \mathrm{NH}\right), 5.00\left(\mathrm{dd},{ }^{3} J_{2,1}=4.1,{ }^{3} J_{2,3}=10.8 \mathrm{~Hz}, 1 \mathrm{H}, 2-\mathrm{H}\right), 4.86\left(\mathrm{~d},{ }^{2} J\right.$ $=11.4 \mathrm{~Hz}, 1 \mathrm{H}$, benzyl. H), 4.78-4.73 (m, $2 \mathrm{H}$, benzyl. H), $4.51\left(\mathrm{~d},{ }^{2} J=11.5 \mathrm{~Hz}, 1 \mathrm{H}\right.$, benzyl. $\mathrm{H}), 4.43\left(\mathrm{dd},{ }^{3} J_{3,2}=10.8,{ }^{3} J_{3,4}=2.9 \mathrm{~Hz}, 1 \mathrm{H}, 3-\mathrm{H}\right), 4.31\left(\mathrm{br} \mathrm{d},{ }^{3} J_{\beta, \gamma}=6.5 \mathrm{~Hz}, 1 \mathrm{H}, \beta-\mathrm{H}\right), 4.14$ $\left(\right.$ br d, $\left.{ }^{3} J_{\alpha, \mathrm{NH}}=9.6 \mathrm{~Hz}, 1 \mathrm{H}, \alpha-\mathrm{H}\right), 3.95\left(\mathrm{~d},{ }^{3} J_{3,4}=3.1 \mathrm{~Hz}, 1 \mathrm{H}, 4-\mathrm{H}\right), 3.88\left(\mathrm{t},{ }^{3} J_{5,6}=6.0,{ }^{3} J_{5,6^{\prime}}=\right.$ $6.0 \mathrm{~Hz}, 1 \mathrm{H}, 5-\mathrm{H}), 3.75\left(\mathrm{dd},{ }^{3} J_{5,6}=6.7,{ }^{2} J_{6,6^{\prime}}=11.3 \mathrm{~Hz}, 1 \mathrm{H}, 6-\mathrm{H}\right), 3.53$ (br s, $1 \mathrm{H}, 6$ '-H), 1.47 , $1.46\left(2 \mathrm{~s}, 18 \mathrm{H}, 2 \mathrm{C}_{4} \mathrm{H}_{9}\right), 1.27-1.22\left(\mathrm{~m},{ }^{3} J_{\gamma, \beta}=6.5 \mathrm{~Hz}, 3 \mathrm{H}, \gamma-\mathrm{CH}_{3}\right) .{ }^{13} \mathrm{C}$ NMR $(150.8 \mathrm{MHz}$, $\left.\mathrm{CDCl}_{3}\right): \delta 170.5,169.3,145.1,137.5-128.1(12 \mathrm{C}), 95.6(1-\mathrm{C}), 84.3$ (2-C), 82.8, 80.0, 75.3 (3C), 75.2 ( $\beta-C), ~ 74.8,73.3,72.7$ (4-C), 71.5 (5-C), 61.8 (6-C), $58.0(\alpha-\mathrm{C}), 28.3$ (3 C), 28.0 (3 C), $18.2(\gamma-\mathrm{C})$. MF (FAB): calcd.: $646+23(\mathrm{Na})=669$; found: $669[\mathrm{M}+\mathrm{Na}]^{+}, 819[\mathrm{M}+$ $\mathrm{NaI}] \mathrm{Na}^{+}$. $\mathrm{C}_{33} \mathrm{H}_{46} \mathrm{~N}_{2} \mathrm{O}_{11} \times 0.5 \mathrm{H}_{2} \mathrm{O}(664.75)$ calcd.: $\mathrm{C} 60.44, \mathrm{H} 7.22, \mathrm{~N} 4.27$, found: $\mathrm{C} 60.40, \mathrm{H}$ $7.04, \mathrm{~N} 4.45$. 


\section{0a}

$O$-(2-Acetamido-3,4-di- $O$-benzyl-2-deoxy- $\alpha$-D-galactoypranosyl)- $N$-(tert-

butyloxycarbonyl)-L-serine tert-butyl ester (40a). 39a (1.15 g, $1.82 \mathrm{mmol})$ was dissolved in ethanol $(10 \mathrm{~mL})$ and transferred to a hydrogenation vessel. Platinized Raney nickel $\mathrm{T}_{4}$ catalyst was freshly prepared and the material obtained from $2 \mathrm{~g}$ of Raney nickel/aluminium alloy was suspended in ethanol $(15 \mathrm{~mL})$. From a homogeneous suspension of this catalyst $8 \mathrm{~mL}$ were added to the reaction vessel and the mixture shaken in a Parr apparatus at a hydrogen pressure of $75 \mathrm{psi}$ for $12 \mathrm{~h}$. The catalyst was filtered off and the solvent evaporated. The residue was dissolved in pyridine/acetic anhydride $(2: 1,6 \mathrm{~mL})$ and stirred for $12 \mathrm{~h}$. All volatiles were removed and the crude product purified by flash chromatography (toluene/acetone 2:1) to give the intermediate peracetylated product $(0.98 \mathrm{~g}, 78 \%)$. This material was $O$-deacetylated in dry methanol $(25 \mathrm{~mL})$ with sodium methoxide $(0.0050 \mathrm{~g}, 0.090 \mathrm{mmol})$ within $40 \mathrm{~min}$. The reaction was neutralized by addition of acetic acid and concentrated. Column chromatographic purification of the crude residue (toluene/acetone $2: 1$ ) afforded $40 \mathrm{a}$ as colourless foam $\left(0.92 \mathrm{~g}, 78 \%\right.$ over 3 steps). $R_{\mathrm{f}}=0.50$ (toluene/acetone $\left.3: 2\right) ;[\alpha]_{\mathrm{D}}{ }^{25}=+65.9(\mathrm{c}$ $\left.=1, \mathrm{CHCl}_{3}\right) .{ }^{1} \mathrm{H}$ NMR $\left(600 \mathrm{MHz}, \mathrm{CDCl}_{3}\right): \delta=7.39-7.26(\mathrm{~m}, 10 \mathrm{H}$, arom. $\mathrm{H}), 5.40($ br d, $1 \mathrm{H}$, Ser-NH), $5.29\left(\mathrm{br} \mathrm{d},{ }^{3} J_{\mathrm{NH}, 2}=8.1 \mathrm{~Hz}, 1 \mathrm{H}\right.$, GalNHAc-HH), $4.98\left(\mathrm{~d},{ }^{2} J=11.7 \mathrm{~Hz}, 1 \mathrm{H}\right.$, benzyl. $\mathrm{H}), 4.84\left(\mathrm{~d},{ }^{3} J_{1.2}=3.6 \mathrm{~Hz}, 1 \mathrm{H}, 1-\mathrm{H}\right), 4.76-4.71\left(\mathrm{~m}, 2 \mathrm{H}\right.$, benzyl. H, 2-H), $4.64\left(\mathrm{~d},{ }^{2} J=11.7\right.$ $\mathrm{Hz}, 1 \mathrm{H}$, benzyl. H), 4.49 (d, ${ }^{2} J=12.1 \mathrm{~Hz}, 1 \mathrm{H}$, benzyl. H), 4.35-4.26 (br s, $\left.1 \mathrm{H}, \alpha-\mathrm{H}\right), 3.94$ (s, $1 \mathrm{H}, 4-\mathrm{H}), 3.84-3.71$ (m, 4 H, 2 ß-H, 6-H, 5-H), 3.57-3.55 (m, 2 H, 6'-H, 3-H), 1.90 (s, 3 H, NHAc), 1.45, $1.44\left(2 \mathrm{~s}, 18 \mathrm{H}, 2 \mathrm{C}_{4} \mathrm{H}_{9}\right) .{ }^{13} \mathrm{C} \mathrm{NMR}\left(150.8 \mathrm{MHz}, \mathrm{CDCl}_{3}\right): \delta 169.7,155.2,138.1-$ 127.8 (12 C), 100.0 (1-C), 82.4, 80.1, 77.2 (3-C), 74.2, 72.3 (4-C), 71.7, 71.6 (5-C), 69.6 ( $\beta$ C), 62.4 (6-C), $54.5(\alpha-C), 48.8$ (2-C), 28.3 (3 C), 28.0 (3 C), 23.4. MS (FAB): calcd.: $644+$ $1(\mathrm{H})=645,644+23(\mathrm{Na})=667,644+173(\mathrm{Na}, \mathrm{NaI})=817$; found: $645[\mathrm{M}+\mathrm{H}]^{+}, 667[\mathrm{M}+$ $\mathrm{Na}]^{+}, 817[\mathrm{M}+\mathrm{NaI}+\mathrm{Na}]^{+}$. HRMS (ESI) $\mathrm{m} / z$ calcd for $\left[\mathrm{C}_{34} \mathrm{H}_{48} \mathrm{~N}_{2} \mathrm{O}_{10}+\mathrm{H}\right]^{+} 645.3387$, found 645.3419 .

\section{$41 \mathrm{a}$}

$O$-(3,4-Di-O-benzyl-6-O-tert-butyldiphenylsilyl-2-deoxy-2-nitro- $\alpha$-D-galactopyranosyl)$(1 \rightarrow 6)$-(2-acetamido-3,4-di- $O$-benzyl-2-deoxy- $\alpha$-D-galactopyranosyl)- $N$-(tert-

butyloxycarbonyl)-L-serine tert-butyl ester (41a). $37(0.02 \mathrm{~g}, 0.031 \mathrm{mmol})$ and 40a $(0.0227 \mathrm{~g}, 0.037 \mathrm{mmol})$ were carefully dried and dissolved in dry toluene $(2 \mathrm{~mL})$. The reaction was started by addition of potassium tert-butoxide ( $31 \mu \mathrm{L}$ of a $1 \mathrm{M}$ solution). After stirring at $\mathrm{rt}$ for $18 \mathrm{~h}$ the reaction did not proceed any further and was thus quenched by addition of acetic acid and concentrated. Purification by flash chromatography (toluene/acetone 5:1) afforded 41a as colourless oil $(0.0272 \mathrm{~g} \mathrm{70 \%}) . \mathrm{R}_{\mathrm{f}}=0.43$ (toluene/acetone $\left.3: 1\right) ;[\alpha]_{\mathrm{D}}{ }^{25}=+28.0$ $\left(\mathrm{c}=1, \mathrm{CHCl}_{3}\right) .{ }^{1} \mathrm{H} \mathrm{NMR}\left(600 \mathrm{MHz}, \mathrm{CDCl}_{3}\right): \delta=7.60-7.17\left(\mathrm{~m}, 30 \mathrm{H}\right.$, arom. H), $5.40\left(\mathrm{~d},{ }^{3} J_{\mathrm{NH}, 2}\right.$ $=9.4 \mathrm{~Hz}, 1 \mathrm{H}$, GalNHAc-NH), $5.18\left(\mathrm{~d},{ }^{3} J_{\mathrm{NH}, \alpha}=5.2 \mathrm{~Hz}, 1 \mathrm{H}, \operatorname{Ser}-\mathrm{NH}\right), 5.11\left(\mathrm{~d},{ }^{3} J_{1,2}=4.3 \mathrm{~Hz}\right.$, $1 \mathrm{H}, 1 \mathrm{~b}-\mathrm{H}), 5.00-4.95$ (m, $2 \mathrm{H}, 2 \mathrm{~b}-\mathrm{H}$, benzyl. H); 4.83 (d, ${ }^{2} J=11.0 \mathrm{~Hz}, 1 \mathrm{H}$, benzyl. H), 4.804.70 (m, $5 \mathrm{H}, 3$ benzyl. H, 1a-H, 2a-H), $4.51\left(\mathrm{~d},{ }^{2} \mathrm{~J}=12.2 \mathrm{~Hz}, 1 \mathrm{H}\right.$, benzyl. H), 4.47 (d, ${ }^{2} J=$ $11.0 \mathrm{~Hz}, 1 \mathrm{H}$, benzyl. H), 4.44 (br d, $\left.{ }^{3} J_{3,2}=11.1 \mathrm{~Hz}, 1 \mathrm{H}, 3 \mathrm{~b}-\mathrm{H}\right), 4.39\left(\mathrm{~d},{ }^{2} J=11.5 \mathrm{~Hz}, 1 \mathrm{H}\right.$, benzyl. H), 4.35 (br s, $1 \mathrm{H}, \alpha-\mathrm{H}), 4.00\left(\mathrm{~d},{ }^{3} J_{4,3}=3.1 \mathrm{~Hz}, 1 \mathrm{H}, 4 \mathrm{~b}-\mathrm{H}\right), 3.87\left(\mathrm{t},{ }^{3} J_{5,6}=6.7 \mathrm{~Hz}\right.$, $\left.{ }^{3} J_{5,6^{\prime}}=6.7 \mathrm{~Hz}, 1 \mathrm{H}, 5 \mathrm{~b}-\mathrm{H}\right), 3.83\left(\mathrm{dd},{ }^{2} J_{\beta, \beta^{\prime}}=13.5,{ }^{3} J_{\beta, \alpha}=3.7 \mathrm{~Hz}, 1 \mathrm{H}, \beta-\mathrm{H}\right), 3.78\left(\mathrm{~d},{ }^{3} J_{4,3}=2.4\right.$ $\mathrm{Hz}, 1 \mathrm{H}, 4 \mathrm{a}-\mathrm{H}), 3.75-3.66$ (m, $\left.5 \mathrm{H}, 6 \mathrm{~b}-\mathrm{H}, 6 \mathrm{~b}^{\prime}-\mathrm{H}, 5 \mathrm{a}-\mathrm{H}, \beta^{\prime}-\mathrm{H}, 6 \mathrm{a}-\mathrm{H}\right), 3.57$ (dd, ${ }^{3} J_{3,2}=10.8,{ }^{3} J_{3,4}$ $=2.4 \mathrm{~Hz}, 3 \mathrm{a}-\mathrm{H}), 3.34\left(\mathrm{dd},{ }^{3} J_{6,5}=6.5,{ }^{2} J_{6,6}=10.1 \mathrm{~Hz}, 1 \mathrm{H}, 6 \mathrm{G}-\mathrm{H}\right), 1.93$ (s, $\left.3 \mathrm{H}, \mathrm{NHAc}\right), 1.45$, $1.44\left(2 \mathrm{~s}, 18 \mathrm{H}, 2 \mathrm{C}_{4} \mathrm{H}_{9}\right), 1.04\left(\mathrm{~s}, 9 \mathrm{H}, \mathrm{C}_{4} \mathrm{H}_{9}\right) .{ }^{13} \mathrm{C} \mathrm{NMR}\left(150.8 \mathrm{MHz}, \mathrm{CDCl}_{3}\right): \delta=169.8$, 155.2, 138.3-127.5 (36 C), 99.1 (1a-C), 96.3 (1b-C), 84.5 (2b-C), 77.0 (3a-C), 75.1 (2 C, 3bC, benzyl. C), 74.2, 73.2, 73.1 (4b-C), 72.2 (4a-C), 71.4, 71.2 (5b-C), 69.7 (5a-C), 68.4 ( $\beta-C)$, 66.7 (6a-C), 61.9 (6b-C), 54.3 ( $\alpha-C), 48.6$ (2a-C), 28.3 (3 C), 28.0 (3 C), 26.8 (3 C), 23.4, 
19.1. MS (FAB): calcd. $1254+23(\mathrm{Na})=1277,1254+39(\mathrm{~K})=1293$; found $1277[\mathrm{M}+$ $\mathrm{Na}]^{+}, 1293[\mathrm{M}+\mathrm{K}]^{+}$. HRMS (ESI) $\mathrm{m} / z$ calcd for $\left[\mathrm{C}_{70} \mathrm{H}_{87} \mathrm{~N}_{3} \mathrm{O}_{16}+\mathrm{Na}\right]^{+} 1276.5748$, found 1276.5704 .

43

$O$-(3,4-Di- $O$-benzyl-6- $O$-( $t$-butyldiphenylsilyl)-2-deoxy-2-nitro- $\alpha$-D-galactopyranosyl)$(\mathbf{1} \rightarrow \mathbf{3})$-6-O-(triisopropylsilyl)-D-galactal (43). To a mixture of $2(200 \mathrm{mg}, 0.66 \mathrm{mmol})$ in dry toluene $(10 \mathrm{~mL})$ was added potassium tert-butoxide $(0.521 \mathrm{M}$ solution in THF); the reaction mixture was stirred for $10 \mathrm{~min}$ at $\mathrm{rt}$ and then added $37(400 \mathrm{mg}, 0.65 \mathrm{mmol})$. The reaction was stopped after $3 \mathrm{~h}$ by adding acetic acid and the solvents were evaporated in vacuo. Purification by flash chromatography (petroleum ether/ethyl acetate 9:1) furnished 43 (420 $\mathrm{mg}, 0.46 \mathrm{mmol}, 70 \%)$ as colourless oil. $\mathrm{R}_{\mathrm{f}}=0.68$ (petroleum ether/ethyl acetate $\left.3: 1\right) ;[\alpha]_{\mathrm{D}}(\mathrm{c}$ $\left.=1, \mathrm{CHCl}_{3}\right)=+22.2 .{ }^{1} \mathrm{H}$ NMR $\left(600 \mathrm{MHz}, \mathrm{CDCl}_{3}\right): \delta=7.64-7.62(\mathrm{~m}, 4 \mathrm{H}$, arom. H), 7.42$7.20\left(\mathrm{~m}, 16 \mathrm{H}\right.$, arom. H), $6.34\left(\mathrm{~d}, J_{1,2}=6.3 \mathrm{~Hz}, 1 \mathrm{H}, 1 \mathrm{a}-\mathrm{H}\right), 5.48\left(\mathrm{~d}, J_{1,2}=4.2 \mathrm{~Hz}, 1 \mathrm{H}, 1 \mathrm{~b}-\mathrm{H}\right)$, $5.04\left(\mathrm{dd}, J_{2,3}=10.4 \mathrm{~Hz}, J_{2,1}=4.2 \mathrm{~Hz}, 1 \mathrm{H}, 2 \mathrm{~b}-\mathrm{H}\right), 4.86(\mathrm{~d}, J=11.3 \mathrm{~Hz}, 1 \mathrm{H}$, benzyl. H), 4.804.74 (m, $2 \mathrm{H}$, benzyl. H), 4.55-4.49 (m, $3 \mathrm{H}, 2 \mathrm{a}-\mathrm{H}, 3 \mathrm{~b}-\mathrm{H}$, benzyl. H), $4.28(\mathrm{~m}, 1 \mathrm{H}, 3 \mathrm{a}-\mathrm{H})$, 4.04-3.94 (m, 4 H, 4a-H, 6a-H, 4b-H, 5b-H), 3.92 (m, 1 H, 6a'-H), 3.87 (m, 1 H, 5b-H), 3.78$3.73\left(\mathrm{~m}, 2 \mathrm{H}\right.$, benzyl. H), $2.20(\mathrm{~d}, J=3.9 \mathrm{~Hz}, 1 \mathrm{H}, \mathrm{OH}) 1.16-0.98(\mathrm{~m}, 30 \mathrm{H}$, TIPS, t-Bu $) .{ }^{13} \mathrm{C}$ NMR (151 MHz, $\mathrm{CDCl}_{3}$ ): $\delta=145.5$ (C-1a), 135.5-127.9 (24 C, arom. C), 99.3 (C-2a), 95.9 (C-1b), 84.6 (C-2b), 77.2 (C-5a), 75.3 (C-3b), 73.4 (C-4b), 71.8 (C-3a), 71.9 (C-5b), 62.9 (C4b), 62.2 (C-6b), 61.9 (C-6a), 26.8-11.7 (15 C). MALDI-MS (positive mode, DHB): [M + $\mathrm{Na}]^{+}: m / z$ calcd.: 935.4 , found: 934.9. $\mathrm{C}_{51} \mathrm{H}_{69} \mathrm{NO}_{10} \mathrm{Si}_{2}$ (850.13) calcd.: C: $67.13, \mathrm{H}: 7.64, \mathrm{~N}$ : 1.53, found: C: $67.27, \mathrm{H}: 7.55, \mathrm{~N}: 1.09$.

44

$O$-(3,4-Di- $O$-benzyl-6- $O$-( $t$-butyldiphenylsilyl)-2-deoxy-2-nitro- $\alpha$-D-galactopyranosyl)$(\mathbf{1} \rightarrow 3)-4-O$-acetyl-6-O -(triisopropylsilyl)-D-galactal (44). A solution of 43 (1.3 g, 1.42 $\mathrm{mmol})$ in $60 \mathrm{~mL}$ pyridine/ $/ \mathrm{Ac}_{2} \mathrm{O}(2: 1)$ was stirred for $16 \mathrm{~h}$ at $\mathrm{rt}$. Removal of the solvent in vacuo and purification by flash chromatography (toluene/ethyl acetate $=10: 1$ ) furnished 44 $(1.21 \mathrm{~g}, 1.26 \mathrm{mmol}, 89 \%)$ as colourless oil. $\mathrm{R}_{\mathrm{f}}=0.62$ (petroleum ether/ethyl acetate $\left.6: 1\right) ;[\alpha]_{\mathrm{D}}$ $\left(\mathrm{c}=1, \mathrm{CHCl}_{3}\right)=+38.9 .{ }^{1} \mathrm{H} \mathrm{NMR}\left(250 \mathrm{MHz}, \mathrm{CDCl}_{3}\right): \delta=7.64-7.61(\mathrm{~m}, 4 \mathrm{H}$, arom. H), 7.42$7.21\left(\mathrm{~m}, 16 \mathrm{H}\right.$, arom. H), $6.30\left(\mathrm{~d}, J_{1,2}=6.3 \mathrm{~Hz}, 1 \mathrm{H}, 1 \mathrm{a}-\mathrm{H}\right), 5.35\left(\mathrm{~d}, J_{1,2}=4.2 \mathrm{~Hz}, 1 \mathrm{H}, 1 \mathrm{~b}-\mathrm{H}\right)$, $5.20\left(\mathrm{dd}, J_{4,3}=J_{4,5}=3.9 \mathrm{~Hz}, 1 \mathrm{H}, 4 \mathrm{a}-\mathrm{H}\right), 4.94\left(\mathrm{dd}, J_{2,1}=J_{2,3}=3.9 \mathrm{~Hz}, 1 \mathrm{H}, 2 \mathrm{~b}-\mathrm{H}\right), 4.87-4.71$ (m, $2 \mathrm{H}$, benzyl. H), 4.59-4.53 (m, $1 \mathrm{H}, 2 \mathrm{a}-\mathrm{H}), 4.50(\mathrm{~d}, J=12.2 \mathrm{~Hz}, 2 \mathrm{H}$, benzyl. H), 4.40 $\left(\mathrm{dd}, J_{3,2}=9.6 \mathrm{~Hz}, J_{3,4}=3.9 \mathrm{~Hz}, 1 \mathrm{H}, 3 \mathrm{~b}-\mathrm{H}\right), 4.26-4.23(\mathrm{~m}, 1 \mathrm{H}, 3 \mathrm{a}-\mathrm{H}), 4.15-4.12(\mathrm{~m}, 1 \mathrm{H}, 5 \mathrm{a}-$ H), 4.08-3.89 (m, 3 H, 4b-H, 5b-H, 6a-H), 3.76-3.69 (m, 3 H, 6a-H, 6a'-H, 6b'-H), 2.10 (s, 3 $\mathrm{H}$, OAc) $1.15-1.00\left(\mathrm{~m}, 30 \mathrm{H}\right.$, TIPS, t-Bu). ${ }^{13} \mathrm{C} \mathrm{NMR}\left(151 \mathrm{MHz}, \mathrm{CDCl}_{3}\right): \delta=170.2(\mathrm{Ac}-\mathrm{C})$, 144.7 (C-1a), 137.8-127.7 (m, 24 C), 99.6 (C-2a), 96.1 (C-1b), 83.9 (C-2b), 76.4 (C-5a), 75.5 (C-3b), 75.0, 73.3 (C-4b), 73.3, 71.6 (C-5b), 67.6 (C-3a), 65.2 (C-4a), 62.3 (C-6b), 60.6 (C6a), 48.2, 26.8, 20.4, 19.1, 18.0, 12.1. MALDI-MS (positive mode, DHB): $[\mathrm{M}+\mathrm{Na}]^{+}: \mathrm{m} / \mathrm{z}$ calcd.: 976.0, found: 976.3. $\mathrm{C}_{53} \mathrm{H}_{71} \mathrm{NO}_{11} \mathrm{Si}_{2}\left(954.31 \times \mathrm{H}_{2} \mathrm{O}\right)$, calcd.: C: $65.47, \mathrm{H}: 7.57, \mathrm{~N}$ : 1.44, found: C: $65.56, \mathrm{H}: 7.47, \mathrm{~N}: 1.78$.

45

$O$-(3,4-Di- $O$-benzyl-6- $O$-(t-butyldiphenylsilyl)-2-deoxy-2-nitro- $\alpha$-D-galactopyranosyl)$(1 \rightarrow 3)-4-O$-acetyl-2-deoxy-2-nitro-6-O-(triisopropylsilyl)-D-galactal (45). Following general procedure A from $44(720 \mathrm{mg}, 0.81 \mathrm{mmol})$, after flash chromatography with toluene/ethyl acetate (10:1) compound $45(690 \mathrm{mg}, 0.69 \mathrm{mmol}, 85 \%)$ was obtained as slightly 
yellow oil. $\mathrm{R}_{\mathrm{f}}=0.62$ (petroleum ether/ethyl acetate 6:1); $[\alpha]_{\mathrm{D}}\left(\mathrm{c}=1, \mathrm{CHCl}_{3}\right)=+41.0 .{ }^{1} \mathrm{H}$ $\operatorname{NMR}\left(600 \mathrm{MHz}, \mathrm{CDCl}_{3}\right): \delta=8.08(\mathrm{~s}, 1 \mathrm{H}, 1 \mathrm{a}-\mathrm{H}), 7.64-7.61(\mathrm{~m}, 4 \mathrm{H}$, arom. H), 7.42-7.16 (m, $16 \mathrm{H}$, arom. H), $5.53\left(\mathrm{~d}, J_{1,2}=4.4 \mathrm{~Hz}, 1 \mathrm{H}, 1 \mathrm{~b}-\mathrm{H}\right), 5.19$ (br s, $\left.1 \mathrm{H}, 4 \mathrm{a}-\mathrm{H}\right), 5.07(\mathrm{~d}, J=3.3 \mathrm{~Hz}$, $1 \mathrm{H}, 3 \mathrm{a}-\mathrm{H}), 4.98\left(\mathrm{dd}, J_{2,1}=4.4, J_{2,3}=10.6 \mathrm{~Hz}, 1 \mathrm{H}, 2 \mathrm{~b}-\mathrm{H}\right), 4.83(\mathrm{~d}, J=10.7 \mathrm{~Hz}, 1 \mathrm{H}$, benzyl. $\mathrm{H})$, 4.76-4.72 (m, $2 \mathrm{H}$, benzyl. H), 4.50 (d, $J=10.7 \mathrm{~Hz}, 1 \mathrm{H}$, benzyl. H), 4.46 (br s, $1 \mathrm{H}, 5 \mathrm{a}-$ H), $4.37\left(\mathrm{dd}, J_{3,2}=10.6, J_{3,4}=1.7 \mathrm{~Hz}, 1 \mathrm{H}, 3 \mathrm{~b}-\mathrm{H}\right), 4.15(\mathrm{~s}, 1 \mathrm{H}, 5 \mathrm{~b}-\mathrm{H}), 3.99(\mathrm{br} \mathrm{s}, 1 \mathrm{H}, 5 \mathrm{a}-\mathrm{H})$, 3.94-3.87 (m, 2 H, 6a-H, 6a'-H), 3.79 (d, J=10.7 Hz, 2 H, 6a-H, 6a'-H), 2.14 (s, 3 H, OAc) 1.11-1.04 (m, $30 \mathrm{H}$, TIPS, t-Bu). ${ }^{13} \mathrm{C}$ NMR (151 MHz, $\mathrm{CDCl}_{3}$ ): $\delta=169.6$ (Ac-C), $155.3(\mathrm{C}-$ 1a), 138.0-127.7 (24 C, arom. C), 96.4 (C-1b), 83.7 (C-2b), 79.0 (C-5a), 75.1 (C-3b), 73.0 (C4b), 718 (C-5b), 65.6 (C-4a), 65.0 (C-3a), 60.6 (C-6a), 60.6 (C-6b), 26.9, 20.4, 19.1, 18.0, 12.0 (13 C). MALDI-MS (positive mode, DHB): $[\mathrm{M}+\mathrm{Na}]^{+}: \mathrm{m} / z$ calcd.: 1023.3, found: 1024.2. $\mathrm{C}_{53} \mathrm{H}_{70} \mathrm{~N}_{2} \mathrm{O}_{13} \mathrm{Si}_{2}$ (999.31), calcd.: C: 63.70, H: 7.06, N: 2.80, found: C: 63.49, H: 7.00, $\mathrm{N}: 2.87$.

\section{6a}

$O$-(3,4-Di- $O$-benzyl-6- $O$-( $t$-butyldiphenylsilyl)-2-deoxy-2-nitro- $\alpha$-D-galactopyranosyl)$(1 \rightarrow 3)-4-O$-acetyl-2-deoxy-2-nitro-6-O-(triisopropylsilyl)- $\alpha$-D-galactopyranosyl)- $N$ - $(t$ -

butyloxycarbonyl)-L-serine tert-butylester (46a). A solution of 44 (200 $\mathrm{mg}, 0.2 \mathrm{mmol})$ and $7 \mathbf{a}(130 \mathrm{mg}, 0.5 \mathrm{mmol})$ in dry toluene $(10 \mathrm{~mL})$ was stirred and potassium tert-butoxide $(3 \mathrm{mg}$, $0.02 \mathrm{mmol}$ ) added. After $2 \mathrm{~h}$ the reaction was neutralized with acetic acid and then the solvent removed in vacuo. Purification by flash chromatography (petroleum ether/ethyl acetate $=6: 1$ ) furnished 46a (181 mg, $0.15 \mathrm{mmol}, 74 \%)$ as colourless oil. $\mathrm{R}_{\mathrm{f}}=0.65$ (petroleum ether/ethyl acetate 6:1 $) ;[\alpha]_{\mathrm{D}}\left(\mathrm{c}=0.25, \mathrm{CHCl}_{3}\right)=+6.0 .{ }^{1} \mathrm{H} \mathrm{NMR}\left(600 \mathrm{MHz}, \mathrm{CDCl}_{3}\right): \delta=7.69(\mathrm{~d}, J=6.7$ $\mathrm{Hz}, 1 \mathrm{H}$, arom. H) $7.64(\mathrm{~d}, J=6.7 \mathrm{~Hz}, 4 \mathrm{H}$, arom. H), 7.41-7.11 (m, $16 \mathrm{H}$, arom. H), 5.55 (d, $\left.J_{1,2}=4.3 \mathrm{~Hz}, 1 \mathrm{H}, 1 \mathrm{~b}-\mathrm{H}\right), 5.52(\mathrm{~d}, J=3.9 \mathrm{~Hz}, 1 \mathrm{H}, 4 \mathrm{a}-\mathrm{H}), 5.37\left(\mathrm{~d}, J_{1,2}=4.2 \mathrm{~Hz}, 1 \mathrm{H}, 1 \mathrm{a}-\mathrm{H}\right)$, $5.10(\mathrm{~d}, J=8.3 \mathrm{~Hz}, 1 \mathrm{H}, \mathrm{NH}), 4.98\left(\mathrm{dd}, J_{2,1}=4.2, J_{2,3}=10.8 \mathrm{~Hz}, 1 \mathrm{H}, 2 \mathrm{~b}-\mathrm{H}\right), 4.83-4.76(\mathrm{~m}, 3$ H, 2a-H, 3a-H, benzyl. H), $4.70(\mathrm{~s}, 1 \mathrm{H}$, benzyl. H), 4.45 (d, $J=10.9 \mathrm{~Hz}, 2 \mathrm{H}$, benzyl. H), $4.31\left(\mathrm{dd}, J_{3,2}=10.8, J_{3,4}=12.6 \mathrm{~Hz}, 1 \mathrm{H}, 3 \mathrm{~b}-\mathrm{H}\right), 4.24-4.20$ (m, $\left.2 \mathrm{H}, 5 \mathrm{~b}-\mathrm{H}, \alpha-\mathrm{Ser}-\mathrm{H}\right), 4.05$ (s, 1 $\mathrm{H}, 4 \mathrm{~b}-\mathrm{H}), 3.91-3.81$ (m, $\left.4 \mathrm{H}, 5 \mathrm{~b}-\mathrm{H}, 6 \mathrm{a}^{\prime}-\mathrm{H}, \beta-S e r-H, \beta^{\prime}-\mathrm{Ser}-\mathrm{H}\right), 3.73 \mathrm{dd}, J_{6,5}=J_{6,6^{\prime}}=8.4 \mathrm{~Hz}, 1$ H, 6a'-H), 3.64 (d, J =6.3 Hz, 1 H, 6b'-H), 2.10 (s, $3 \mathrm{H}, \mathrm{OAc}), 1.43$ ( $2 \times \mathrm{s}, 18 \mathrm{H}, \mathrm{t}-\mathrm{Bu}, \mathrm{Boc})$, 1.11-1.04 (m, $30 \mathrm{H}$, TIPS, t-Bu). ${ }^{13} \mathrm{C}$ NMR (151 MHz, $\left.\mathrm{CDCl}_{3}\right): \delta=135.6-127.8$ (24 C, arom. C), 96.5 (C-1a), 91.6 (C-1b), 83.9 (C-2b), 83.2 (C-2a), 75.3 (C-3b), 73.2 (C-4b), 72.3 (C-5a), 70.7 (C-5b), 68.9 (C-3), 67.9 (C-3a), 64.0 (C-4a), 61.9 (C-6a), 61.9 (C-6b), 28.3, 27.8, 26.8, 17.8, 11.8 (24 C). MALDI-MS (positive mode, DHB): $[\mathrm{M}+\mathrm{Na}]^{+}: \mathrm{m} / z$ calcd.: 1283.1, found: 1284.1. $\mathrm{C}_{65} \mathrm{H}_{93} \mathrm{~N}_{3} \mathrm{O}_{18} \mathrm{Si}_{2}$ (1260.66), calcd.: C: 61.93, H: 7.44, N: 3.33, found: C: 61.86, H: $7.57, \mathrm{~N}: 3.23$.

\section{9}

1,5-Anhydro-3,6-di- $O$-benzyl-2-deoxy-D-arabino-hex-1-enitol (49). 48 ${ }^{[11]}$ (53 g, 0.117 mol) was dissolved in THF $(250 \mathrm{~mL})$ and treated with tetrabutylammonium fluoride $(352 \mathrm{~mL}$ of a 1 $\mathrm{M}$ solution in THF) at $\mathrm{rt}$. The reaction mixture was stirred for $2 \mathrm{~h}$ and then concentrated and dried under high vacuum. The crude material was redissolved in DMF (300 mL) and cooled in an ice-bath. Sodium hydride $(8.44 \mathrm{~g}, 0.352 \mathrm{~mol})$ was slowly added under constant stirring. After hydrogen formation ceased, benzyl bromide $(41.8 \mathrm{~mL}, 0.352 \mathrm{~mol})$ was slowly dropped to the reaction mixture. Cooling was removed and stirring continued for $12 \mathrm{~h}$. The reaction was quenched with triethylamine $(20 \mathrm{~mL})$ and concentrated. The organic material was extracted with diethyl ether from diethyl ether/water, dried over sodium sulfate and the solvent removed. The crude product was purified on silica gel (toluene/ethyl acetate 10:1) to give intermediate 3,6-di-O-benzyl- $O$-4-tetrahydropyranyl-D-glucal. This material was in turn dissolved in THF $(150 \mathrm{~mL})$ and treated with acetic acid $(300 \mathrm{~mL})$ and water $(80 \mathrm{~mL})$. The reaction mixture was stirred at $\mathrm{rt}$ for $24 \mathrm{~h}$. The reaction was diluted with diethyl ether $(500$ 
$\mathrm{mL})$ and water $(120 \mathrm{~mL})$ and the organic material extracted to the ether phase. Drying over sodium sulfate, concentration and column chromatographic purification (toluene/ethyl acetate 5:1) gave 49 as colourless oil $(24 \mathrm{~g}, 63 \%)$. The analytical data are in accordance with those reported. ${ }^{[25]}$

\section{0}

1,5-Anhydro-3,6-di- $O$-benzyl-4- $O$-tert-butyldimethylsilyl-2-deoxy-D-arabino-hex-1-enitol (50). 49 (1.39 g, $4.26 \mathrm{mmol})$ was dissolved in DMF (15 mL) and imidazole (0.72 g, 10.65 $\mathrm{mmol})$ and then TBDMSCl (1.28 g, $8.52 \mathrm{mmol})$ was added. The reaction mixture was stirred for $12 \mathrm{~h}$ at $\mathrm{rt}$, diluted with diethyl ether $(50 \mathrm{~mL})$ and the organic layer washed with water $(50$ $\mathrm{mL})$. Drying over sodium sulfate, concentration and column chromatographic purification (toluene) afforded 50 as colourless oil $(1.57 \mathrm{~g}, 84 \%) . \mathrm{R}_{\mathrm{f}}=0.27$ (toluene); $[\alpha]_{\mathrm{D}}=-15.4(\mathrm{c}=$ 0.5, $\left.\mathrm{CHCl}_{3}\right) .{ }^{1} \mathrm{H}$ NMR $\left(600 \mathrm{MHz}, \mathrm{CDCl}_{3}\right): \delta=7.33-7.27\left(\mathrm{~m}, 10 \mathrm{H}\right.$, arom. H), $6.41\left(\mathrm{~d},{ }^{3} J_{1,2}=\right.$ $6.3 \mathrm{~Hz}, 1 \mathrm{H}, 1-\mathrm{H}), 4.83\left(\mathrm{dd},{ }^{3} J_{1,2}=6.3,{ }^{3} J_{2,3}=2.7 \mathrm{~Hz}, 1 \mathrm{H}, 2-\mathrm{H}\right), 4.63\left(\mathrm{~d},{ }^{2} J=12.2 \mathrm{~Hz}, 1 \mathrm{H}\right.$, benzyl. H), 4.59 (d, ${ }^{2} J=11.6 \mathrm{~Hz}, 1 \mathrm{H}$, benzyl. H), $4.53\left(\mathrm{~d},{ }^{2} \mathrm{~J}=12.2 \mathrm{~Hz}, 1 \mathrm{H}\right.$, benzyl. H), 4.49 $\left(\mathrm{d},{ }^{2} J=11.6 \mathrm{~Hz}, 1 \mathrm{H}\right.$, benzyl. H), 3.99-3.93 (m, $\left.3 \mathrm{H}, 3-\mathrm{H}, 4-\mathrm{H}, 5-\mathrm{H}\right), 3.77\left(\mathrm{dd},{ }^{3} J_{6,5}=2.5,{ }^{3} J_{6,6}\right.$ $=10.6 \mathrm{~Hz}, 1 \mathrm{H}, 6-\mathrm{H}), 3.71\left(\mathrm{dd},{ }^{3} J_{6,5}=6.3,{ }^{3} J_{6,6}=10.6 \mathrm{~Hz}, 6{ }^{\prime}-\mathrm{H}\right), 0.82\left(\mathrm{~s}, 9 \mathrm{H}, \mathrm{C}_{4} \mathrm{H}_{9}\right), 0.05$, $0.03\left(2 \mathrm{~s}, 6 \mathrm{H}, 2 \mathrm{SiCH}_{3}\right) .{ }^{13} \mathrm{C} \mathrm{NMR}\left(150.8 \mathrm{MHz}, \mathrm{CDCl}_{3}\right): \delta=144.7$ (1-C), 138.4, 138.1, 128.3-127.5 (10 C), 99.3 (2-C), 78.3 (5-C), 76.8 (3-C), 73.4, 70.0, 68.9 (6-C), 68.6 (4-C), 25.8, 18.1, -4.2, -5.1. MS (FAB): calcd.: $440+23(\mathrm{Na})=463$; found $463[\mathrm{M}+\mathrm{Na}]^{+}$. $\mathrm{C}_{26} \mathrm{H}_{36} \mathrm{O}_{4} \mathrm{Si}$ (440.24), calcd.: C: 70.87, H: 8.23, found: C: 71.04, H: 8.39.

\section{1}

1,5-Anhydro-3,6-di-O-benzyl-4- $O$-tert-butyldimethylsilyl-2-deoxy-2-nitro-D-arabino-hex1-enitol (51). Following general procedure A from $50(0.25 \mathrm{~g}, 0.567 \mathrm{mmol})$, after flash chromatography with toluene/ethyl acetate (98:2) compound $\mathbf{5 1}(0.149 \mathrm{~g}, 54 \%)$ was obtained as slightly yellow oil. $\mathrm{R}_{\mathrm{f}}=0.53$ (toluene/ethyl acetate 98:2); $[\alpha]_{\mathrm{D}}=-4.6\left(\mathrm{c}=1, \mathrm{CHCl}_{3}\right) .{ }^{1} \mathrm{H}$ $\operatorname{NMR}\left(600 \mathrm{MHz}, \mathrm{CDCl}_{3}\right): \delta=8.21(\mathrm{~s}, 1 \mathrm{H}, 1-\mathrm{H}), 7.35-7.25\left(\mathrm{~m}, 10 \mathrm{H}\right.$, arom. H) $4.71\left(\mathrm{~d},{ }^{2} J=\right.$ $11.4 \mathrm{~Hz}, 1 \mathrm{H}$, benzyl. H), $4.65\left(\mathrm{~d},{ }^{2} J=11.4 \mathrm{~Hz}, 1 \mathrm{H}\right.$, benzyl. H), $4.51\left(\mathrm{~d},{ }^{2} J=11.9 \mathrm{~Hz}, 1 \mathrm{H}\right.$, benzyl. H), 4.49-4.45 (m, $3 \mathrm{H}$, benzyl. H, 4-H, 5-H), $4.12\left(\mathrm{t},{ }^{3} J_{3,4}=2.2, J_{3,1}<1 \mathrm{~Hz}, 1 \mathrm{H}, 3-\mathrm{H}\right)$, $3.71\left(\mathrm{dd},{ }^{3} J_{6,5}=8.1,{ }^{2} J_{6,6^{\prime}}=10.8 \mathrm{~Hz}, 1 \mathrm{H}, 6-\mathrm{H}\right), 3.64\left(\mathrm{dd},{ }^{3} J_{6,5}=5.2,{ }^{2} J_{6^{\prime}, 6}=10.8 \mathrm{~Hz}, 1 \mathrm{H}, 6^{\prime}-\right.$ $\mathrm{H}), 0.81\left(\mathrm{~s}, 9 \mathrm{H}, \mathrm{C}_{4} \mathrm{H}_{9}\right), 0.01,-0.01\left(2 \mathrm{~s}, 6 \mathrm{H}, 2 \mathrm{SiCH}_{3}\right) .{ }^{13} \mathrm{C} \mathrm{NMR}\left(150.8 \mathrm{MHz}, \mathrm{CDCl}_{3}\right): \delta=$ 154.4, 137.6, 137.5, 130.6-127.7 (10 C), 81.3 (5-C), 73.5, 73.0, 70.5 (4-C), 67.6 (6-C), 65.6 (3-C), 25.5, -4.9, -5.1. MS (FAB): calcd.: $485+23(\mathrm{Na})=508$; found $486[\mathrm{M}+\mathrm{H}]^{+}, 508[\mathrm{M}$ $+\mathrm{Na}]^{+}$. HRMS (ESI) $\mathrm{m} / z$ calcd for $\left[\mathrm{C}_{26} \mathrm{H}_{35} \mathrm{NO}_{6} \mathrm{Si}+\mathrm{Na}\right]^{+}$508.2120, found 508.2118.

\section{2}

1,5-Anhydro-3,4-di-O-tert-butyldimethylsilyl-2-deoxy-D-lyxo-hex-1-enitol (52). D-Galactal $(5 \mathrm{~g}, 34.2 \mathrm{mmol})$ was dissolved in pyridine $(20 \mathrm{~mL})$ and cooled to $-20{ }^{\circ} \mathrm{C}$. To this solution pivaloyl chloride ( $4.2 \mathrm{~mL}, 34.2 \mathrm{mmol}$ ) was slowly added over $10 \mathrm{~min}$ under constant stirring. Stirring was continued for $2 \mathrm{~h}$ and the reaction then quenched by addition of methanol $(1 \mathrm{~mL})$. The reaction mixture was concentrated and the crude material purified by flash chromatography (chloroform/methanol 12:1 containing 5\% triethylamine). Intermediate 6-Opivaloyl-galactal $(3.1 \mathrm{~g})$ was redissolved in DMF $(15 \mathrm{~mL})$ and treated with imidazole $(2.66 \mathrm{~g}$, $39.1 \mathrm{mmol})$ and TBDMSCl $(4.91 \mathrm{~g}, 32.6 \mathrm{mmol})$ and the reaction mixture was stirred at $\mathrm{rt}$ for $12 \mathrm{~h}$. The reaction was diluted with diethyl ether and washed with water. Concentration of the organic layer and flash chromatography of the crude product (toluene) gave intermediate 3,4di-O-tert-butyldimethylsilyl-6-O-pivaloyl-galactal. This material $(3.2 \mathrm{~g})$ was dissolved in dichloromethane $(50 \mathrm{~mL})$ and the solution cooled to $-70{ }^{\circ} \mathrm{C}$. At this temperature DIBAL-H solution $\left(21.0 \mathrm{~mL}\right.$ of a $1 \mathrm{M}$ solution in $\mathrm{CH}_{2} \mathrm{Cl}_{2}$ ) was slowly added to the reaction. Methanol (5 $\mathrm{mL}$ ) was used to quench the reaction. The mixture was poured into a saturated sodium 
bicarbonate solution and the organic material extracted with $\mathrm{CH}_{2} \mathrm{Cl}_{2}$. Drying of the organic layer over sodium sulfate, concentration and flash chromatography (toluene/ethyl acetate 5:1) yielded $\mathbf{5 2}$ as colourless oil $\left(2.3 \mathrm{~g}, 18 \%\right.$ over three steps). $\mathrm{R}_{\mathrm{f}}=0.38$ (toluene/ethyl acetate $5: 1) ;[\alpha]_{\mathrm{D}}=+7.5\left(\mathrm{c}=0.25, \mathrm{CHCl}_{3}\right) .{ }^{1} \mathrm{H} \mathrm{NMR}\left(600 \mathrm{MHz}, \mathrm{CDCl}_{3}\right): \delta=6.33\left(\mathrm{~d},{ }^{3} J_{1,2}=6.1 \mathrm{~Hz}\right.$, $1 \mathrm{H}, 1-\mathrm{H}), 4.67\left(\mathrm{t},{ }^{3} J_{2,1}=5.6,{ }^{3} J_{2,3}=5.6 \mathrm{~Hz}, 1 \mathrm{H}, 2-\mathrm{H}\right), 4.17\left(\mathrm{t},{ }^{3} J_{4,3}=4.5,{ }^{3} J_{4,5}=4.5 \mathrm{~Hz}, 1 \mathrm{H}\right.$, $4-\mathrm{H}), 4.12\left(\mathrm{t},{ }^{3} J_{3,2}=4.5,{ }^{3} J_{3,4}=4.5 \mathrm{~Hz}, 1 \mathrm{H}, 3-\mathrm{H}\right), 4.08-4.06(\mathrm{~m}, 2 \mathrm{H}, 5-\mathrm{H}, 6-\mathrm{H}), 3.85-3.80(\mathrm{~m}$, $1 \mathrm{H}, 6$ '- $\mathrm{H}), 0.92,0.91\left(2 \mathrm{~s}, 18 \mathrm{H}, 2 \mathrm{C}_{4} \mathrm{H}_{9}\right), 0.11\left(\mathrm{~s}, 12 \mathrm{H}, 4 \mathrm{SiCH}_{3}\right) .{ }^{13} \mathrm{C}$ NMR $(150.8 \mathrm{MHz}$, $\mathrm{CDCl}_{3}$ ): $\delta=144.4$ (1-C), 100.9 (2-C), 69.3 (4-C), 64.2 (3-C), 60.4 (6-C), 25.9, 25.8, 18.1, 18.1, -4.3, -4.5, -4.8, -5.0. MS (EI): calcd.: 375; found: 375. HRMS (ESI) $\mathrm{m} / \mathrm{z}$ calcd for $\left[\mathrm{C}_{18} \mathrm{H}_{38} \mathrm{O}_{4} \mathrm{Si}+\mathrm{K}\right]^{+}$413.1941, found 413.1947 .

\section{3}

$O$-(3,6-Di-O-benzyl-4-O-tert-butyldimethylsilyl-2-deoxy-2-nitro- $\beta$-D-glucopyranosyl)$(1 \rightarrow 6)$-1,5-anhydro-3,4-di-O-tert-butyldimethylsilyl-2-deoxy-D-lyxo-hex-1-enitol (53). 51 $(0.12 \mathrm{~g}, 0.25 \mathrm{mmol})$ and $52(0.078 \mathrm{~g}, 0.25 \mathrm{mmol})$ were thoroughly dried under high vacuum and dissolved in dry THF $(5 \mathrm{~mL})$. Potassium tert-butoxide solution $(0.25 \mathrm{~mL}$ of a $1 \mathrm{M}$ solution in THF) was added under constant stirring at $\mathrm{rt}$. The reaction was stirred for $2 \mathrm{~h}$ and then acidified by addition of acetic acid. The mixture was concentrated and the product isolated after flash chromatography (toluene) furnishing $\mathbf{5 3}$ as colourless oil $(0.135 \mathrm{~g}, 63 \%)$. $\mathrm{R}_{\mathrm{f}}=0.76$ (toluene/ethyl acetate 98:2); $[\alpha]_{\mathrm{D}}=-12.7\left(\mathrm{c}=1, \mathrm{CHCl}_{3}\right) .{ }^{1} \mathrm{H} \mathrm{NMR}\left(600 \mathrm{MHz}, \mathrm{CDCl}_{3}\right): \delta=$ 7.34-7.23 (m, $10 \mathrm{H}$, arom. H), $6.18\left(\mathrm{~d},{ }^{3} J_{1,2}=6.1 \mathrm{~Hz}, 1 \mathrm{H}, 1 \mathrm{a}-\mathrm{H}\right), 4.91\left(\mathrm{~d},{ }^{3} J_{1,2}=8.1 \mathrm{~Hz}, 1 \mathrm{H}\right.$, 1b-H), $4.67\left(\mathrm{~d},{ }^{3} J_{2,3}=10.8 \mathrm{~Hz}, 1 \mathrm{H}, 2 \mathrm{a}-\mathrm{H}\right), 4.59\left(\mathrm{~d},{ }^{2} \mathrm{~J}=12.2 \mathrm{~Hz}, 1 \mathrm{H}\right.$, benzyl. H), 4.55-4.50 (m, $2 \mathrm{H}$, benzyl. H, 2b-H), $4.46\left(\mathrm{~d},{ }^{2} \mathrm{~J}=10.8 \mathrm{~Hz}, 1 \mathrm{H}\right.$, benzyl. H), 4.13-4.04 (m, $5 \mathrm{H}, 3 \mathrm{a}-\mathrm{H}$, 3b-H, 5a-H, 6a-H, 6'a-H), 3.97 (br s, $1 \mathrm{H}, 4 \mathrm{a}-\mathrm{H}), 3.73\left(\mathrm{t},{ }^{3} J_{4,3}=8.9,{ }^{3} J_{4,5}=8.9 \mathrm{~Hz}, 1 \mathrm{H}, 4 \mathrm{~b}-\mathrm{H}\right)$, 3.68-3.63 (m, 2 H, 6b-H, 6'b-H), 3.50-3.49 (m, $1 \mathrm{H}, 5 \mathrm{~b}-\mathrm{H}), 0.89,0.88,0.86$ (3 s, $27 \mathrm{H}, 3$ $\left.\mathrm{C}_{4} \mathrm{H}_{9}\right), 0.09-0.03\left(6 \mathrm{~s}, 18 \mathrm{H}, 6 \mathrm{SiCH}_{3}\right) .{ }^{13} \mathrm{C} \mathrm{NMR}\left(151 \mathrm{MHz}, \mathrm{CDCl}_{3}\right): \delta=142.4(\mathrm{C}-1 \mathrm{a}), 138.1-$ 127.5 (m, 10 C), 102.6 (C-2a), 89.9 (C-2b), 82.2 (C-3b), 76.9 (C-5a), 76.8 (C-5b), 74.9, 73.4, 70.4 (C-4b), 68.7 (C-4a), 68.1 (C-6b), 67.5 (C-6a), 64.1 (C-3a), 26.1, 25.8, 18.1, 18.0, -3.8, 4.3. MS (FAB): calcd.: $859+23(\mathrm{Na})=882$; found $882[\mathrm{M}+\mathrm{Na}]^{+}$. HRMS (ESI) $\mathrm{m} / z$ calcd for $\left[\mathrm{C}_{44} \mathrm{H}_{73} \mathrm{NO}_{10} \mathrm{Si}+\mathrm{Na}\right]^{+} 882.4434$, found 882.4420.

\section{4}

$O$-(3,6-Di-O-benzyl-4- $O$-tert-butyldimethylsilyl-2-deoxy-2-nitro- $\beta$-D-glucopyranosyl)$(1 \rightarrow 6)$-1,5-anhydro-3,4-di- $O$-tert-butyldimethylsilyl-2-deoxy-2-nitro-D-lyxo-hex-1-enitol

(54). Following general procedure A from $53(0.11 \mathrm{~g}, 0.13 \mathrm{mmol})$, after flash chromatography with toluene/ethyl acetate (10:1) compound $54(0.052 \mathrm{~g}, 45 \%)$ was obtained as slightly yellow oil. $\mathrm{R}_{\mathrm{f}}=0.52$ (toluene/ethyl acetate 98:2); $[\alpha]_{\mathrm{D}}=+9.7\left(\mathrm{c}=0.25, \mathrm{CHCl}_{3}\right) .{ }^{1} \mathrm{H} \mathrm{NMR}(600 \mathrm{MHz}$, $\left.\mathrm{CDCl}_{3}\right): \delta=8.04(\mathrm{~s}, 1 \mathrm{H}, 1 \mathrm{a}-\mathrm{H}), 7.32-7.22(\mathrm{~m}, 10 \mathrm{H}$, arom. H), 4.85-4.83 (m, $2 \mathrm{H}, 1 \mathrm{~b}-\mathrm{H}), 3 \mathrm{a}-$ $\mathrm{H}), 4.67\left(\mathrm{~d},{ }^{2} J=10.8 \mathrm{~Hz}\right.$, benzyl. H), $4.57\left(\mathrm{~d},{ }^{2} J=12.2 \mathrm{~Hz}, 1 \mathrm{H}\right.$, benzyl. H), $4.53\left(\mathrm{dd},{ }^{3} J_{2,1}=\right.$ $\left.8.2,{ }^{3} J_{2,3}=1.7 \mathrm{~Hz}, 1 \mathrm{H}, 2 \mathrm{~b}-\mathrm{H}\right), 4.48-4.42\left(\mathrm{~m}, 3 \mathrm{H}, 2\right.$ benzyl. H, 5a-H), $4.26\left(\mathrm{~d},{ }^{2} J_{6,6^{\prime}}=12.5 \mathrm{~Hz}\right.$, $1 \mathrm{H}, 6 \mathrm{a}-\mathrm{H}), 4.10-4.00(\mathrm{~m}, 3 \mathrm{H}, 3 \mathrm{~b}-\mathrm{H}, 4 \mathrm{a}-\mathrm{H}), 6 \mathrm{a}-\mathrm{H}), 3.75\left(\mathrm{t},{ }^{3} \mathrm{~J}_{4,3}=8.9,{ }^{3} J_{4,5}=8.8 \mathrm{~Hz}, 1 \mathrm{H}, 4 \mathrm{~b}-\right.$ H), 3.65 (m, 2 H, 6b-H, 6'b-H), 3.48-3.46 (m, $1 \mathrm{H}, 5 \mathrm{~b}-\mathrm{H}), 0.89-0.79\left(3 \mathrm{~s}, 27 \mathrm{H}, 3 \mathrm{C}_{4} \mathrm{H}_{9}\right), 0.16-$ $0.00\left(6 \mathrm{~s}, 18 \mathrm{H}, 6 \mathrm{SiCH}_{3}\right) .{ }^{13} \mathrm{C} \mathrm{NMR}\left(150.8 \mathrm{MHz}, \mathrm{CDCl}_{3}\right): \delta=153.8(1 \mathrm{a}-\mathrm{C}), 138.0,136.9$, 133.2, 128.3-127.3 (10 C), 100.4 (1b-C), 35.7 (2a-C), 89.6 (2b-C), 82.1 (3b-C), 79.3 (5a-C), 76.8 (5b-C), 75.0, 73.5, 70.3 (4b-C), 68.1 (6a-C), 68.0 (6b-C), 68.0 (4a-C), 63.8 (3a-C), 26.225.8 (6 C), 18.3, 18.3, 17.9, -3.6, -4.4, -4.9, -5.0, -5.1 (2 C). MS (FAB): calcd.: $904+23(\mathrm{Na})$ $=927$; found $927[\mathrm{M}+\mathrm{Na}]^{+} . \mathrm{HRMS}(\mathrm{ESI}) \mathrm{m} / z$ calcd for $\left[\mathrm{C}_{44} \mathrm{H}_{72} \mathrm{~N}_{2} \mathrm{O}_{12} \mathrm{Si}_{3}+\mathrm{Na}\right]^{+} 927.4285$, found 927.4309 . 